\title{
Vibration Attenuation of Composite Moving Beams using Active Vibration Control Techniques
}

\author{
Gouthami Polina \\ West Virginia University
}

Follow this and additional works at: https://researchrepository.wvu.edu/etd

\section{Recommended Citation}

Polina, Gouthami, "Vibration Attenuation of Composite Moving Beams using Active Vibration Control Techniques" (2014). Graduate Theses, Dissertations, and Problem Reports. 651.

https://researchrepository.wvu.edu/etd/651

This Thesis is protected by copyright and/or related rights. It has been brought to you by the The Research Repository @ WVU with permission from the rights-holder(s). You are free to use this Thesis in any way that is permitted by the copyright and related rights legislation that applies to your use. For other uses you must obtain permission from the rights-holder(s) directly, unless additional rights are indicated by a Creative Commons license in the record and/ or on the work itself. This Thesis has been accepted for inclusion in WVU Graduate Theses, Dissertations, and Problem Reports collection by an authorized administrator of The Research Repository @ WVU. For more information, please contact researchrepository@mail.wvu.edu. 


\title{
Vibration Attenuation of Composite Moving Beams using Active Vibration Control Techniques
}

\section{Gouthami Polina}

\author{
Thesis submitted to the \\ at the West Virginia University \\ Master of Science \\ in \\ Mechanical Engineering \\ Nithi Sivaneri, Ph.D., Chair \\ Victor Mucino, Ph.D. \\ Roxana Cisloiu, Ph.D.
}

Stattler College of Engineering and Mineral resources in partial fulfillment of the requirements for the Degree of

Department of Mechanical and Aerospace Engineering Morgantown, West Virginia

April 2014 


\section{ABSTRACT \\ Vibration Attenuation of Composite Moving Beams using Active Vibration Control Techniques}

\section{Gouthami Polina}

Applications of axially-moving beams are found in earthquake engineering, robotic arms, conveyor belts, etc. Previous studies have shown that the amplitude of lateral vibration of moving beams is 40 percent higher than that of non moving beams. In this research a numerical model based on the finite element method is done to reduce the excess vibrations caused by the axial oscillation of the beam. A computer code is written in MATLAB to accomplish this.

The vibrational amplitude of composite moving beams is reduced by means of an active vibration control technique that employs the piezoelectric effect. Piezoelectric sensors and actuators are added to the beam to sense and control the vibrational response of the moving beam. The sensors and actuators are made to communicate with each other using negative velocity feedback control. Classical laminate plate theory (CLPT), first order shear deformation theory (FSDT) and higher order shear deformation theories (HSDT) are considered for the analysis of composite beams. A consistent formulation is used to reduce the composite plate theory to beams. The governing equations are obtained using variational principles. The displacement constarints are applied through Lagrange multipliers. An over hanging beam is considered for the analysis pupose. First bending mode shape is taken as the initial shape of the beam. Newmark's time integration scheme is used to generate the controlled response of the beam. The feedback gains are altered to have a desired control at certain time.

Results are presented in terms of tip deflections. The damped response of the CLPT is compared with that of FSDT and HSDT for a specific gain. A parametric study is conducted by varying the frequency of axial oscillation and considering different laminates. 
Dedicated to my mother, Prasanthi and father, Satyanarayana 


\section{ACKNOWLEDGEMENTS}

I would like to thank my advisor, Dr Nithi Sivaneri, from the bottom of my heart for providing guidance throughout my graduate education at WVU. I have learnt a lot from him, both professionally and personally, and could not have asked for a better mentor. His passion for teaching is very inspiring and helped me understand the concepts to the core. He has always encouraged me to learn new methods and techniques in mechanics by providing invaluable resources. His suggestions have helped me a lot in improving my problem solving skills and explore new ways for implementing solutions.

I would also like to thank Dr. Victor Mucino for helping me during my research. I am also thankful to Dr Roxana Cisliou for giving me an opportunity to work as co-op at ANSYS. She has also encouraged me to work on projects, providing me an experience that was instrumental in successful completion of my research. My experience at ANSYS has contributed a lot to my professional development and exposure to the industry.

I am very glad to be around wonderful people during my stay at WVU. I have learnt a lot of important things in life, which were very helpful in overcoming tough times, from my friends and peers. I would like to thank my roommates and friends for their moral support during my research. I am grateful to my aunt and uncle, Janaki and Murali Krishna, who were responsible for making me feel home during my initial struggles in united states. It was my aunt's initiative that has laid the foundation for my journey at WVU and gave me the strength to reach where I am now. I am indebted to my parents, Satyanarayana and Prasanthi, for their unconditional love and support. Although the professional challenges were inspiring and motivating it was only because of their support that I could overcome the personal challenges. 


\section{TABLE OF CONTENTS}

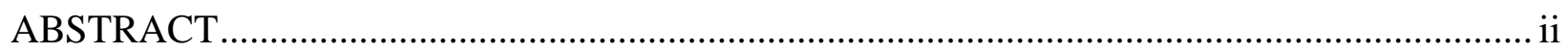

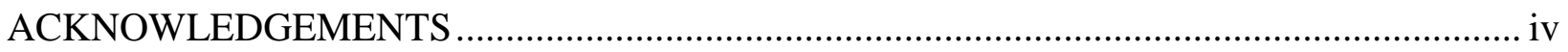

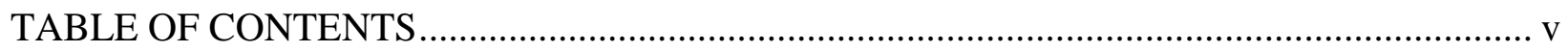

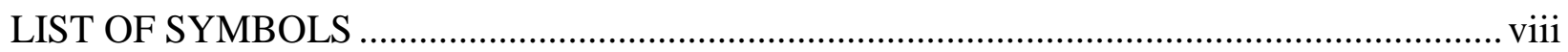

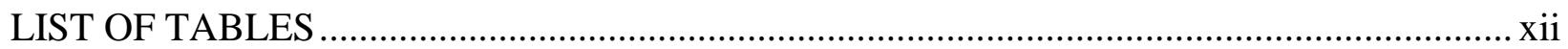

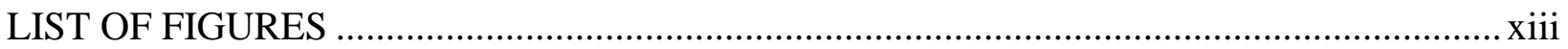

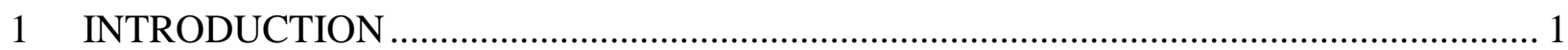

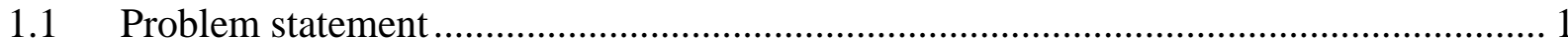

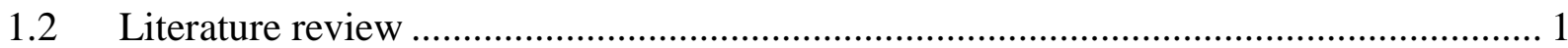

1.2.1 Isotropic moving beams ............................................................................ 1

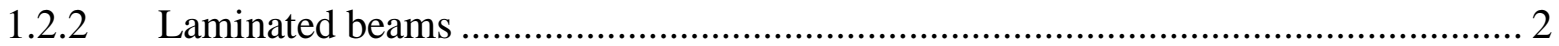

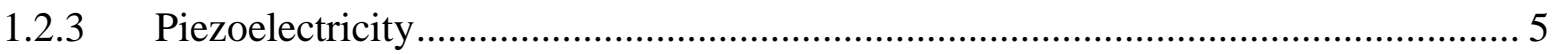

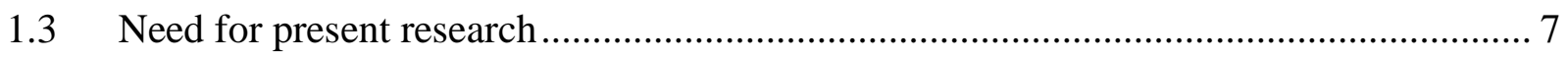

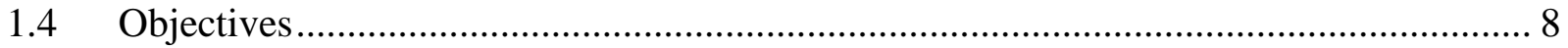

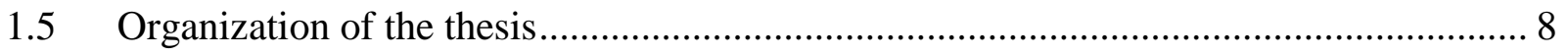

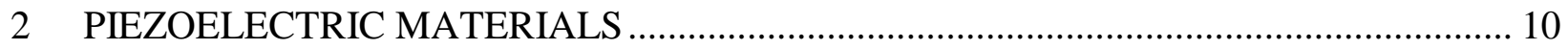

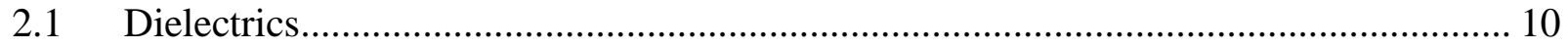

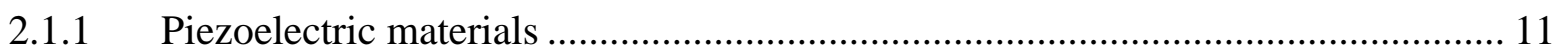

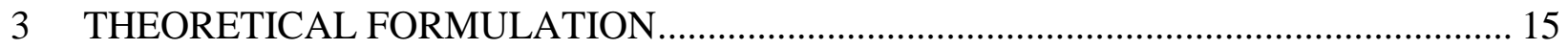

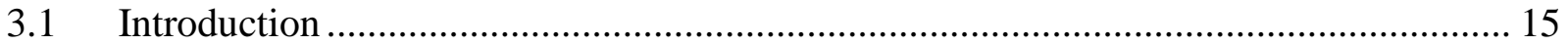

3.2 Moving Beam Coordinate Systems ……………………………………………..... 16

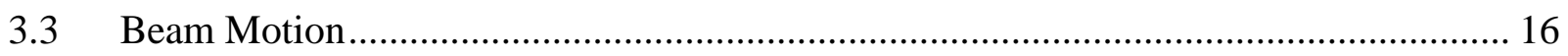

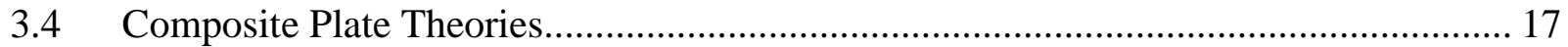

3.4.1 Classical Laminate Plate theory ...................................................................... 17

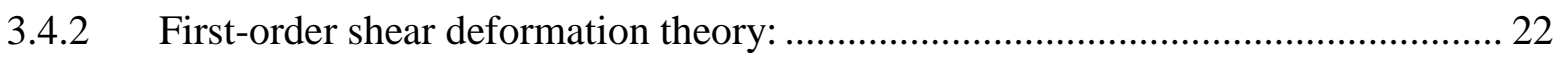

3.4.3 Higher order shear deformation theory ……………............................................ 26

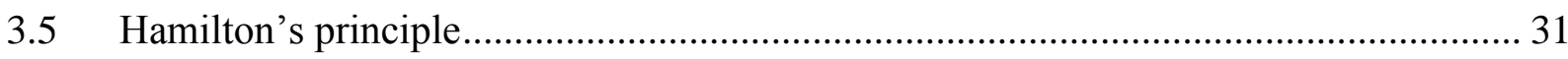

3.6 Virtual mechanical strain energy for the plate …………........................................... 32 


\subsubsection{Reduction of Plate Equations to Beam in a Consistent Manner for CLPT}

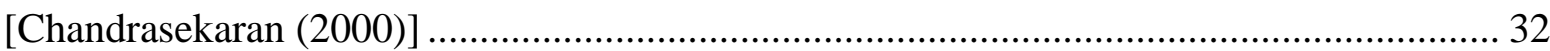

3.6.2 Reduction of Plate Equations to Beam in a Consistent Manner for FSDT............ 35

3.6.3 Reduction of Plate Equations to Beam in a Consistent Manner for HSDT

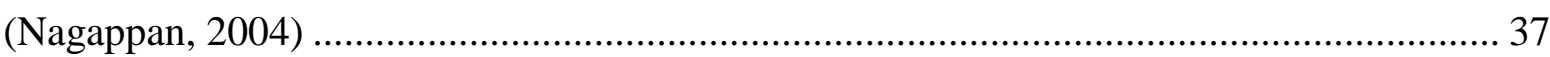

3.7 Virtual electrical strain energy of a composite plate ................................................ 41

3.7.1 Virtual electrical strain energy of a composite beam using CLPT ...................... 42

3.7.2 Virtual electrical strain energy of a composite beam using FSDT: ..................... 43

3.7.3 Virtual electrical strain energy of a composite beam using HSDT: .................... 43

3.8 Virtual Kinetic energy of a composite beam [Nagappan (2004)] .............................. 44

3.8.1 Virtual Kinetic energy of a composite beam using CLPT: ................................ 44

3.8.2 Virtual Kinetic energy of a composite beam for using FSDT: ........................... 46

3.8.3 Virtual kinetic energy of a composite beam for partial plane stress using HSDT.. 48

$3.9 \quad$ Virtual work done on a composite plate............................................................... 51

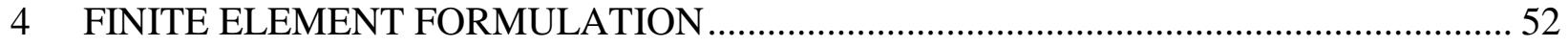

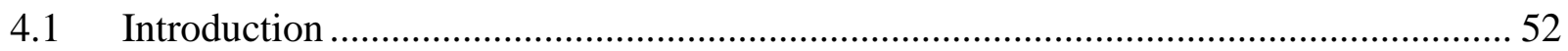

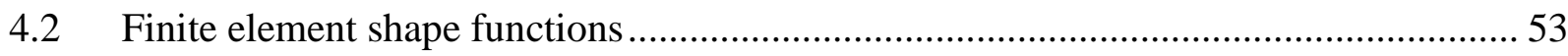

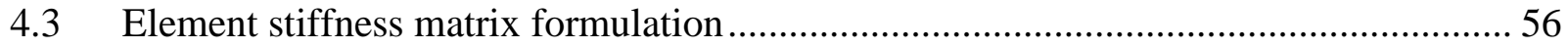

4.3.1 Element Stiffness matrix for beam using CLPT .............................................. 56

4.3.2 Element Stiffness Matrix for beam using FSDT ............................................ 60

4.3.3 Element Stiffness matrix for reduced beam formulation using HSDT ................. 64

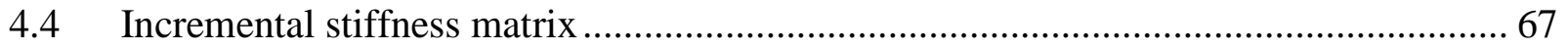

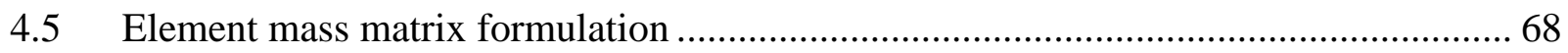

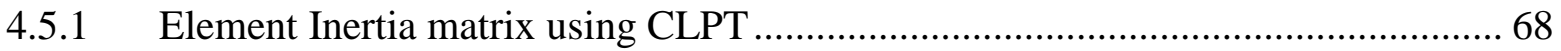

4.5.2 Element Inertia matrix for beam using FSDT ............................................ 69

4.5.3 Element Inertia matrix for beam using HSDT ............................................. 71

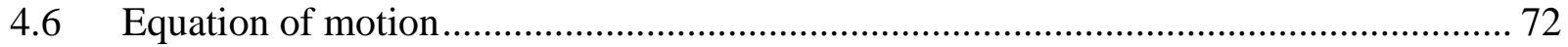

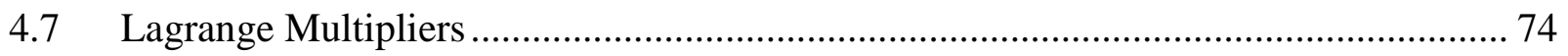

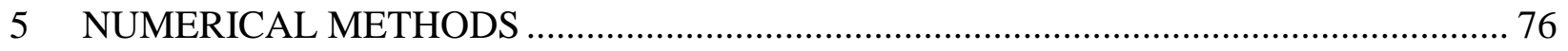

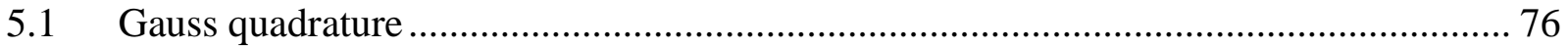

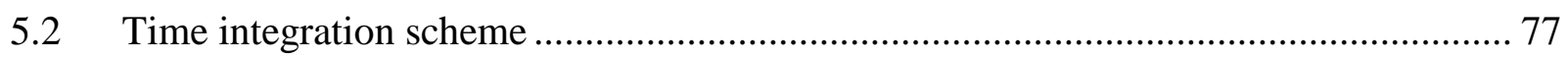


5.2.1 Step by step procedure for the Newmark method ........................................ 78

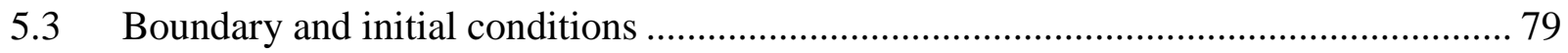

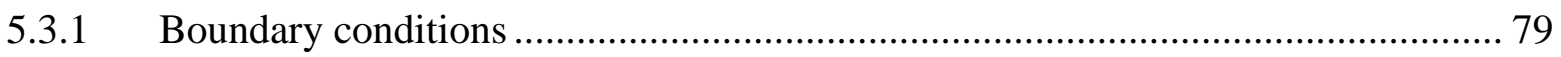

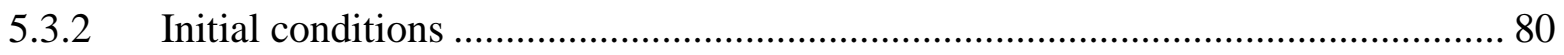

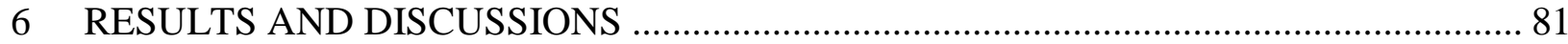

6.1 Verification of Composite moving beam ................................................................ 81

6.2 Verification of piezoelectric formulation for a non moving beam.............................. 86

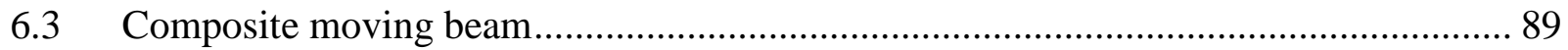

6.3.1 Moving beam simulation using CLPT ........................................................ 90

6.3.2 Moving beam simulation using FSDT .......................................................... 113

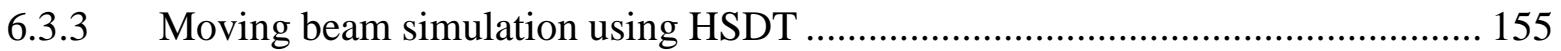

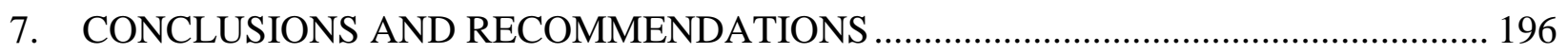

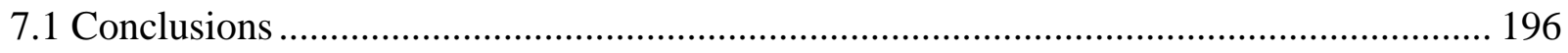

7.2 Recommendations for Future Work .................................................................... 197

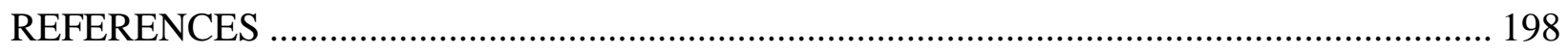

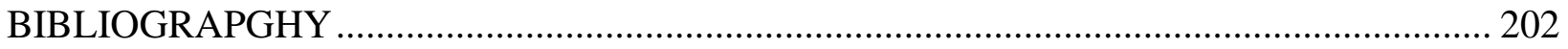




\section{LIST OF SYMBOLS}

$a_{B}^{L}$

A

$[A]$

$a_{i}, b_{j}$

b

[B]

$\left[C_{e}\right]$

[C]

$d$

[d]

$\{D\}$

$[D]$

$\mathrm{e}$

$\bar{e}$

E

$\left\{E_{i}\right\}$

$[E],[F],[H]$

$G$

h

$H_{i}, H_{L i}$

I

$I_{0}, I_{1}, I_{2}$

$I_{3}, I_{4}, I_{6}$

$K$

[K $\left.K_{x x}\right]$

$\left[K_{S S}\right]$

$\left[K_{S \varphi}\right]$

$\left[K_{\varphi \varphi}\right]$

- Longitudinal rigid-body acceleration of the beam $\left(\mathrm{m} / \mathrm{s}^{2}\right)$

- Amplitude of longitudinal rigid-body motion (m)

- Extension stiffness matrix (N/m)

- Generalized coordinates

- Width of the beam (m)

- Bending-extension coupling stiffness matrix $(\mathrm{N})$

- Finite Element damping matrix

- Global damping matrix

- Distance between supports (m)

- Dielectric constant matrix $(\mathrm{m} / \mathrm{V})$

- Dielectric displacement $\left(\mathrm{C} / \mathrm{m}^{2}\right)$ or $(\mathrm{N} / \mathrm{V} \mathrm{m})$

- Bending stiffness matrix ( $\mathrm{N} \mathrm{m})$

- Piezoelectric modulus $\left(\mathrm{C} / \mathrm{m}^{2}\right)$ or $(\mathrm{N} / \mathrm{V} \mathrm{m})$

- Transformed piezoelectric modulus $\left(\mathrm{C} / \mathrm{m}^{2}\right)$ or $(\mathrm{N} / \mathrm{V} \mathrm{m})$

- Modulus of elasticity $\left(\mathrm{N} / \mathrm{m}^{2}\right)$

- Electric field (V/m)

- Higher-order stiffness matrices $\left(\mathrm{N} \mathrm{m}^{2}, \mathrm{~N} \mathrm{~m}^{3}, \mathrm{~N} \mathrm{~m}^{5}\right)$

- Feedback gain.

- Thickness of the beam (m)

- Hermitian and Lagrangian Shape functions

- Moment of Inertia $\left(\mathrm{m}^{4}\right)$

- Normal, coupled normal-rotary, and rotary inertia coefficients

- Higher-order inertia coefficients

- Shear correction factor

- Finite element stiffness matrix for composite material

- Elastic stiffness matrix

- Piezoelectric coupling stiffness matrix

- Electrical stiffness matrix 
$[\widehat{K}]$

$[K]$

$\left[K_{\lambda}\right]$

$L$

$l_{e}$

$\left[M_{s s}\right]$

$\left[M_{x x}\right]$

$[M]$

$M_{x}, M_{y}, M_{x y}$

$M_{x}^{P}, M_{y}^{P}, M_{x y}^{P}$

$n$

$N_{x}, N_{y}, N_{x y}$

$N_{x}^{P}, N_{y}^{P}, N_{x y}^{P}$

$P_{x}, P_{y}, P_{x y}$

$\{q\}$

$Q_{x}, Q_{y}$

$\left[\bar{Q}^{k}\right]$

$\{Q\}$

$[\mathrm{S}]^{\mathrm{ij}}$

$\left[\bar{S}_{i j}\right]$

$\left\{\bar{S}_{P}\right\}_{a},\left\{\bar{S}_{P}\right\}_{s}$

$t$

$t_{k}$

$t_{p}$

$T$
- Modified elastic stiffness matrix

- Global stiffness matrix

- Lagrange multiplier matrix

- Length of the beam (m)

- Element length (m)

- Inertial matrix corresponding to the elastic terms.

- Finite element inertia matrix for composite material

- Global Inertia matrix

- In plane moment resultants in $x y$ plane $(\mathrm{Nm})$

- In plane piezoelectric moment resultants in x-y plane

- Number of layers

- In plane force resultants in $x y$ plane $(\mathrm{N} / \mathrm{m})$

- In plane piezoelectric force resultants in $x-y$ plane

- Higher order stress resultants

- Global displacements vector (m)

- Transverse shear force resultants $(\mathrm{N} / \mathrm{m})$

- Transformed reduced stiffness matrix of $k^{\text {th }}$ layer $\left(\mathrm{N} / \mathrm{m}^{2}\right)$

- Global load vector $(\mathrm{N})$

- Partitions of reduced $[A B D]$ matrix in formulation using CLPT and FSDT.

- Element of reduced $[S]$ matrix in formulation based on CLPT and FSDT

- Elements of the reduced piezoelectric stress resultants based on CLPT and FSDT

- Time (s)

- Thickness of $k^{\text {th }}$ layer (m)

- Thickness of piezoelectric layer (m)

- Total kinetic energy (N m) 


\begin{tabular}{|c|c|}
\hline$[T]^{i j}$ & - Partitions of reduced $[A B D E F]$ matrix in formulation using HSDT \\
\hline$\left[\bar{T}_{i j}\right]$ & - Elements of reduced $[T]$ matrix in formulation based on HSDT \\
\hline$\left\{\bar{T}_{P}\right\}_{a},\left\{T_{P}\right\}_{S}$ & - Elements of the reduced piezoelectric stress resultants based on HSDT \\
\hline$u$ & - Axial deflection (m) \\
\hline$u_{0}, v_{0}, w_{0}$ & - Mid-plane displacements along $x, y$, and $z$ axes, respectively (m) \\
\hline$U$ & - Total strain energy ( $\mathrm{N} \mathrm{m})$ \\
\hline$U_{M}$ & - Total Mechanical strain energy ( $\mathrm{N} \mathrm{m})$ \\
\hline$U_{E}$ & - Total electrical strain energy $(\mathrm{N} \mathrm{m})$ \\
\hline$V$ & - Volume $\left(\mathrm{m}^{3}\right)$ \\
\hline$V_{B}^{L}$ & - Longitudinal rigid-body velocity of the beam (m/s) \\
\hline$w_{b}, w_{s}$ & - Shear and bending components of transverse deflection (m) \\
\hline$w$ & - Transverse deflection (m) \\
\hline$W$ & - Work done ( $\mathrm{N} \mathrm{m}$ ) \\
\hline$x_{e}$ & - Element longitudinal axis \\
\hline$X_{A}(t)$ & - Axial displacement of the moving beam \\
\hline$X, Y$ & - Global Axes \\
\hline$z$ & - Thickness coordinate \\
\hline$z_{k}$ & - Distance to the top of the $k^{\text {th }}$ layer from the mid-plane of a laminate $(\mathrm{m})$ \\
\hline$\overline{z_{k}}$ & - Distance to the mid surface of the $k^{\text {th }}$ layer from the mid-plane of a \\
\hline & laminate $(\mathrm{m})$ \\
\hline $\bar{\Delta}_{p}$ & - Hamiltonian function \\
\hline$\Omega$ & - Frequency of rigid-body axial motion imparted to the beam $(\mathrm{rad} / \mathrm{s})$ \\
\hline$\delta()$ & - Variation of ( ) \\
\hline$\varepsilon_{x}, \varepsilon_{y}, \varepsilon_{z}$ & - Normal strains in $x, y$, and $z$, directions, respectively \\
\hline$\epsilon_{i j}$ & - Dielectric permittivity (C/V m ) \\
\hline $\bar{\epsilon}_{i j}$ & - Transformed Dielectric permittivity \\
\hline$\phi_{x}, \phi_{y}$ & - Rotations about $y$ and $x$, respectively \\
\hline$\varphi_{a}, \varphi_{s}$ & - actuator and sensor voltage \\
\hline
\end{tabular}




$\begin{array}{ll}\gamma_{x y}, \gamma_{y z}, \gamma_{x z} & \text { - Engineering shear strains } \\ \rho & \text { - Mass Density }\left(\mathrm{Kg} / \mathrm{m}^{3}\right) \\ \xi & \text { - Element non-dimensional coordinate } \\ ()^{T} & \text { - Transpose of ( ) } \\ \left.()^{\prime},()^{*}\right) & \text { - First and second partial derivatives with respect to time } \\ ()^{\prime},()^{\prime \prime} & \text { - First and second partial derivatives with respect to } x \\ ()^{y} & \text { - Partial derivative with respect to } y\end{array}$




\section{LIST OF TABLES}

Table 5.1 Gauss quadrature sampling points and weights .................................................. 76

Table 6.1 Material properties [Balamurugan and Narayanan (2002)] ...................................... 86

Table 6.2 Material properties for the composite moving beam ................................................ 89

Table 6.3 Geometric properties of the composite moving beam .......................................... 89

Table 6.4 Feedback gains supplied to the beams .......................................................... 92

Table 6.5 Magnification factors for the moving beams with CLPT formulation ...................... 92

Table 6.6 Transverse tip deflection at the left end, $w$, CLPT, $\Omega=10 \mathrm{rad} / \mathrm{s},[0 / \pm 45] \ldots \ldots \ldots \ldots \ldots . . . . .96$

Table 6.7 Transverse tip deflection at the left end, $w$, CLPT, $\Omega=20 \mathrm{rad} / \mathrm{s},[0 / \pm 45] \ldots \ldots \ldots \ldots \ldots . . .100$

Table 6.8 Transverse tip deflection at the left end, $w$, CLPT, $\Omega=10 \mathrm{rad} / \mathrm{s},[0 / \pm 20] \ldots \ldots \ldots \ldots \ldots . . . .104$

Table 6.9 Transverse tip deflection at the left end, $w$, CLPT, $\Omega=20 \mathrm{rad} / \mathrm{s},[0 / \pm 20] \ldots \ldots \ldots \ldots \ldots . . . . .108$

Table 6.10 Transverse tip deflection at the left end, $w$, CLPT, $\Omega=20 \mathrm{rad} / \mathrm{s},[0 / \pm 45]_{\mathrm{s}} \ldots \ldots \ldots \ldots . .112$

Table 6.11 Magnification factors for the moving beams with FSDT formulation .................... 114

Table 6.12 Transverse tip deflection at the left end, $w$, FSDT, $\Omega=10 \mathrm{rad} / \mathrm{s},[0 / \pm 45] \ldots \ldots \ldots \ldots . . .122$

Table 6.13 Transverse tip deflection at the left end, $w$, FSDT, $\Omega=20 \mathrm{rad} / \mathrm{s},[0 / \pm 45] \ldots \ldots \ldots \ldots . . .130$

Table 6.14 Transverse tip deflection at the left end, $w$, FSDT, $\Omega=10 \mathrm{rad} / \mathrm{s},[0 / \pm 20] \ldots \ldots \ldots \ldots . . . .138$

Table 6.15 Transverse tip deflection at the left end, $w$, FSDT, $\Omega=20 \mathrm{rad} / \mathrm{s},[0 / \pm 20] \ldots \ldots \ldots \ldots . . .146$

Table 6.16 Transverse tip deflection at the left end, $w$, FSDT, $\Omega=20 \mathrm{rad} / \mathrm{s},[0 / \pm 45]_{\mathrm{s}} \ldots \ldots \ldots \ldots .154$

Table 6.17 Magnification factors for the moving beams with HSDT formulation ................... 155

Table 6.18 Transverse tip deflection at the left end, $w$, HSDT, $\Omega=10 \mathrm{rad} / \mathrm{s},[0 / \pm 45] \ldots \ldots \ldots \ldots . . .163$

Table 6.19 Transverse tip deflection at the left end, $w$, HSDT, $\Omega=20 \mathrm{rad} / \mathrm{s},[0 / \pm 45] \ldots \ldots \ldots \ldots . .171$

Table 6.20 Transverse tip deflection at the left end, $w$, HSDT, $\Omega=10 \mathrm{rad} / \mathrm{s},[0 / \pm 20] \ldots \ldots \ldots \ldots . . . .179$

Table 6.21 Transverse tip deflection at the left end, $w$, HSDT, $\Omega=20 \mathrm{rad} / \mathrm{s},[0 / \pm 20] \ldots \ldots \ldots \ldots . . . .187$

Table 6.22 Transverse tip deflection at the left end, $w$, HSDT, $\Omega=20 \mathrm{rad} / \mathrm{s},[0 / \pm 45]_{\mathrm{s}} \ldots \ldots \ldots \ldots . . . .195$ 


\section{LIST OF FIGURES}

Figure 2.1 Classification of dielectric materials ................................................................... 12

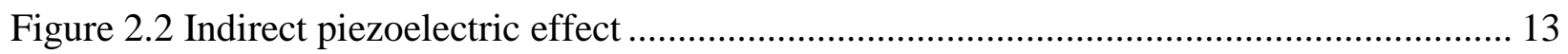

Figure 2.3 Direct piezoelectric effect material ................................................................. 14

Figure 3.1 Coordinate systems for the moving beam ....................................................... 15

Figure 3.2 Undeformed and deformed geometries of an edge of a plate under......................... 18

Figure 3.3 Undeformed and deformed geometries of an edge of a plate under the assumptions of

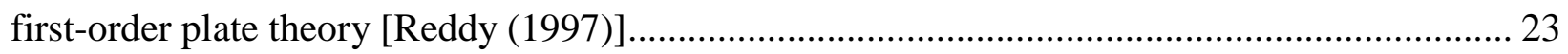

Figure 3.4 Deformation of transverse normal for CLPT, FSDT and HSDT [Reddy (1997)]...... 28

Figure 4.1 Finite element representation of a moving beam ................................................... 53

Figure 4.2 Representation of natural coordinate system of a finite element with three internal

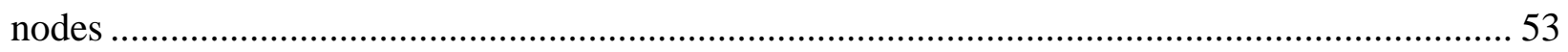

Figure 4.3 Element definition for reduced beam formulation using CLPT ............................... 57

Figure 4.4 Element definition for reduced beam formulation using FSDT ............................. 60

Figure 4.5 Element degrees of freedom for beam formulation using HSDT............................. 64

Figure 6.1 Transverse tip deflection at the left end, $w$, CLPT, $\Omega=20 \mathrm{rad} / \mathrm{s},[0 / \pm 45]_{\mathrm{s}} \ldots \ldots \ldots \ldots \ldots . . . . . .33$

Figure 6.2 Transverse tip deflection at the left end, $w$, FSDT, $\Omega=20 \mathrm{rad} / \mathrm{s},[0 / \pm 45]_{\mathrm{s}} \ldots \ldots \ldots \ldots \ldots . . . . .84$

Figure 6.3 Transverse tip deflection at the left end, $w$, HSDT, $\Omega=20 \mathrm{rad} / \mathrm{s},[0 / \pm 45]_{\mathrm{s}} \ldots \ldots \ldots \ldots \ldots . . . . .85$

Figure 6.4 Cantilever beam with piezoelectric sensors and actuators [Balamurugan and

Narayanan (2002)] .................................................................................................... 86

Figure 6.5 Tip deflection of a cantilever beam with piezoelectric layers on the top and bottom. 88

Figure 6.6 Transverse tip deflection at the left end, $w$, CLPT, $\Omega=10 \mathrm{rad} / \mathrm{s},[0 / \pm 45] \ldots \ldots \ldots \ldots \ldots . . . . .93$

Figure 6.7 Transverse tip deflection at the right end, $w$, CLPT, $\Omega=10 \mathrm{rad} / \mathrm{s},[0 / \pm 45] \ldots \ldots \ldots \ldots . . .94$

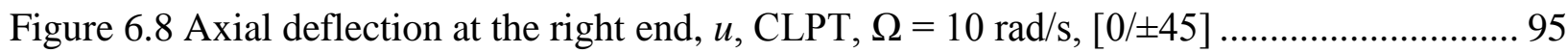

Figure 6.9 Transverse tip deflection at the left end, $w$, CLPT, $\Omega=20 \mathrm{rad} / \mathrm{s},[0 / \pm 45] \ldots \ldots \ldots \ldots \ldots . . . . .97$

Figure 6.10 Transverse tip deflection at the right end, $w$, CLPT, $\Omega=20 \mathrm{rad} / \mathrm{s},[0 / \pm 45] \ldots \ldots \ldots \ldots . . . .98$

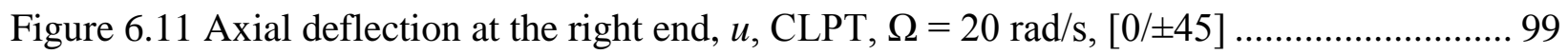

Figure 6.12 Transverse tip deflection at the left end, $w$, CLPT, $\Omega=10 \mathrm{rad} / \mathrm{s},[0 / \pm 20] \ldots \ldots \ldots \ldots .101$

Figure 6.13 Transverse tip deflection at the right end, $w$, CLPT, $\Omega=10 \mathrm{rad} / \mathrm{s},[0 / \pm 20] \ldots \ldots \ldots . .102$

Figure 6.14 Transverse tip deflection at the left end, $w$, CLPT, $\Omega=10 \mathrm{rad} / \mathrm{s},[0 / \pm 20] \ldots \ldots \ldots \ldots . . . .103$

Figure 6.15 Transverse Tip deflection at the left end, $w$, CLPT, $\Omega=20 \mathrm{rad} / \mathrm{sec},[0 / \pm 20] \ldots \ldots \ldots . .105$

Figure 6.16 Transverse tip deflection at the right end, $w$, CLPT, $\Omega=20 \mathrm{rad} / \mathrm{s},[0 / \pm 20] \ldots \ldots \ldots . .106$

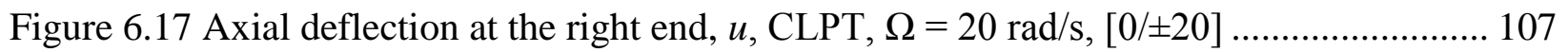

Figure 6.18 Transverse tip deflection at the left end, $w$, CLPT, $\Omega=20 \mathrm{rad} / \mathrm{s},[0 / \pm 45]_{\mathrm{s}} \ldots \ldots \ldots \ldots .109$

Figure 6.19 Transverse tip deflection at the right end, $w$, CLPT, $\Omega=20 \mathrm{rad} / \mathrm{s},[0 / \pm 45]_{\mathrm{s}} \ldots \ldots \ldots .110$

Figure 6.20 Axial deflection at the right end, $u$, CLPT, $\Omega=20 \mathrm{rad} / \mathrm{s},[0 / \pm 45]_{\mathrm{s}} \ldots \ldots \ldots \ldots \ldots \ldots \ldots . . . . . . . . .111$

Figure 6.21 Transverse tip deflection at the left end, $w_{b}$, FSDT, $\Omega=10 \mathrm{rad} / \mathrm{s},[0 / \pm 45] \ldots \ldots \ldots \ldots 115$

Figure 6.22 Transverse tip deflection at the left end, $w_{s}, \mathrm{FSDT}, \Omega=10 \mathrm{rad} / \mathrm{s},[0 / \pm 45] \ldots \ldots \ldots \ldots .116$

Figure 6.23 Transverse tip deflection at the left end, $w$, FSDT, $\Omega=10 \mathrm{rad} / \mathrm{s},[0 / \pm 45] \ldots \ldots \ldots \ldots .117$

Figure 6.24 Transverse tip deflection at the right end, $w_{b}, \mathrm{FSDT}, \Omega=10 \mathrm{rad} / \mathrm{s},[0 / \pm 45] \ldots \ldots \ldots 118$ 
Figure 6.25 Transverse tip deflection at the right end, $w_{s}$, FSDT, $\Omega=10 \mathrm{rad} / \mathrm{s},[0 / \pm 45] \ldots \ldots \ldots .119$

Figure 6.26 Transverse tip deflection at the right end, $w$, FSDT, $\Omega=10 \mathrm{rad} / \mathrm{s},[0 / \pm 45] \ldots \ldots \ldots . .120$

Figure 6.27 Axial deflection at the right end, $u$, FSDT, $\Omega=10 \mathrm{rad} / \mathrm{s},[0 / \pm 45] \ldots \ldots \ldots \ldots \ldots \ldots \ldots . . . . . . .121$

Figure 6.28 Transverse tip deflection at the left end, $w_{b}$, FSDT, $\Omega=20 \mathrm{rad} / \mathrm{s},[0 / \pm 45] \ldots \ldots \ldots \ldots .123$

Figure 6.29 Transverse tip deflection at the left end, $w_{s}$, FSDT, $\Omega=20 \mathrm{rad} / \mathrm{s},[0 / \pm 45] \ldots \ldots \ldots \ldots .124$

Figure 6.30 Transverse tip deflection at the left end, $w$, FSDT, $\Omega=20 \mathrm{rad} / \mathrm{s},[0 / \pm 45] \ldots \ldots \ldots \ldots .125$

Figure 6.31 Transverse tip deflection at the right end, $w_{b}, \mathrm{FSDT}, \Omega=20 \mathrm{rad} / \mathrm{s},[0 / \pm 45] \ldots \ldots \ldots .126$

Figure 6.32 Transverse tip deflection at the right end, $w_{s}$, FSDT, $\Omega=20 \mathrm{rad} / \mathrm{s},[0 / \pm 45] \ldots \ldots \ldots .127$

Figure 6.33 Transverse tip deflection at the right end, $w$, FSDT, $\Omega=20 \mathrm{rad} / \mathrm{s},[0 / \pm 45] \ldots \ldots \ldots . .128$

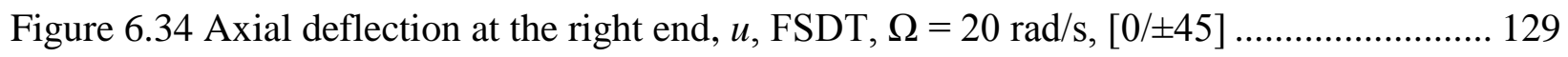

Figure 6.35 Transverse tip deflection at the left end, $w_{b}$, FSDT, $\Omega=10 \mathrm{rad} / \mathrm{s},[0 / \pm 20] \ldots \ldots \ldots \ldots 131$

Figure 6.36 Transverse tip deflection at the left end, $w_{s}$, FSDT, $\Omega=10 \mathrm{rad} / \mathrm{s},[0 / \pm 20] \ldots \ldots \ldots . . .132$

Figure 6.37 Transverse tip deflection at the left end, $w$, FSDT, $\Omega=10 \mathrm{rad} / \mathrm{s},[0 / \pm 20] \ldots \ldots \ldots \ldots .133$

Figure 6.38 Transverse tip deflection at the right end, $w_{b}$, FSDT, $\Omega=10 \mathrm{rad} / \mathrm{s},[0 / \pm 20] \ldots \ldots \ldots 134$

Figure 6.39 Transverse tip deflection at the right end, $w_{s}, \mathrm{FSDT}, \Omega=10 \mathrm{rad} / \mathrm{s},[0 / \pm 20] \ldots \ldots \ldots . . .135$

Figure 6.40 Transverse tip deflection at the right end, $w$, FSDT, $\Omega=10 \mathrm{rad} / \mathrm{s},[0 / \pm 20] \ldots \ldots \ldots . .136$

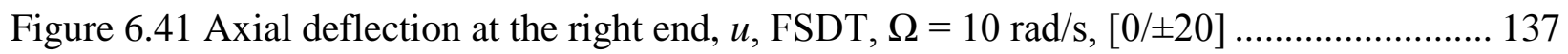

Figure 6.42 Transverse tip deflection at the left end, $w_{b}$, FSDT, $\Omega=20 \mathrm{rad} / \mathrm{s},[0 / \pm 20] \ldots \ldots \ldots . . .139$

Figure 6.43 Transverse tip deflection at the left end, $w_{s}$, FSDT, $\Omega=20 \mathrm{rad} / \mathrm{s},[0 / \pm 20] \ldots \ldots \ldots \ldots .140$

Figure 6.44 Transverse tip deflection at the left end, $w$, FSDT, $\Omega=20 \mathrm{rad} / \mathrm{s},[0 / \pm 20] \ldots \ldots \ldots \ldots .141$

Figure 6.45 Transverse tip deflection at the right end, $w_{b}$, FSDT, $\Omega=20 \mathrm{rad} / \mathrm{s},[0 / \pm 20] \ldots \ldots \ldots .142$

Figure 6.46 Transverse tip deflection at the right end, $w_{s}$, FSDT, $\Omega=20 \mathrm{rad} / \mathrm{s},[0 / \pm 20] \ldots \ldots \ldots .143$

Figure 6.47 Transverse tip deflection at the right end, $w$, FSDT, $\Omega=20 \mathrm{rad} / \mathrm{s},[0 / \pm 20] \ldots \ldots \ldots . .144$

Figure 6.48 Axial deflection at the right end, $u$, FSDT, $\Omega=20 \mathrm{rad} / \mathrm{s},[0 / \pm 20] \ldots \ldots \ldots \ldots \ldots \ldots \ldots . . . . . . . . . .145$

Figure 6.49 Transverse tip deflection at the left end, $w_{b}$, FSDT, $\Omega=20 \mathrm{rad} / \mathrm{s},[0 / \pm 45]_{\mathrm{s}} \ldots \ldots \ldots \ldots .147$

Figure 6.50 Transverse tip deflection at the left end, $w_{s}$, FSDT, $\Omega=20 \mathrm{rad} / \mathrm{s},[0 / \pm 45]_{\mathrm{s}} \ldots \ldots \ldots \ldots .148$

Figure 6.51 Transverse tip deflection at the left end, $w$, FSDT, $\Omega=20 \mathrm{rad} / \mathrm{s},[0 / \pm 45]_{\mathrm{s}} \ldots \ldots \ldots \ldots . . .149$

Figure 6.52 Transverse tip deflection at the right end, $w_{b}$, FSDT, $\Omega=20 \mathrm{rad} / \mathrm{s},[0 / \pm 45]_{\mathrm{s}} \ldots \ldots . .150$

Figure 6.53 Transverse tip deflection at the right end, $w_{s}$, FSDT, $\Omega=20 \mathrm{rad} / \mathrm{s},[0 / \pm 45]_{\mathrm{s}} \ldots \ldots \ldots .151$

Figure 6.54 Transverse tip deflection at the right end, $w_{s}$, FSDT, $\Omega=20 \mathrm{rad} / \mathrm{s},[0 / \pm 45]_{\mathrm{s}} \ldots \ldots \ldots .152$

Figure 6.55 Axial deflection at the right end, $u$, FSDT, $\Omega=20 \mathrm{rad} / \mathrm{s},[0 / \pm 45]_{\mathrm{s}} \ldots \ldots \ldots \ldots \ldots \ldots \ldots . . . . . . . .153$

Figure 6.56 Transverse tip deflection at the left end, $w_{b}, \mathrm{HSDT}, \Omega=10 \mathrm{rad} / \mathrm{s},[0 / \pm 45] \ldots \ldots \ldots . . .156$

Figure 6.57 Transverse tip deflection at the left end, $w_{s}$, HSDT, $\Omega=10 \mathrm{rad} / \mathrm{s},[0 / \pm 45] \ldots \ldots \ldots . . .157$

Figure 6.58 Transverse tip deflection at the left end, $w, \mathrm{HSDT}, \Omega=10 \mathrm{rad} / \mathrm{s},[0 / \pm 45] \ldots \ldots \ldots \ldots .158$

Figure 6.59 Transverse tip deflection at the right end, $w_{b}, \mathrm{HSDT}, \Omega=10 \mathrm{rad} / \mathrm{s},[0 / \pm 45] \ldots \ldots \ldots .159$

Figure 6.60 Transverse tip deflection at the right end, $w_{s}$, HSDT, $\Omega=10 \mathrm{rad} / \mathrm{s},[0 / \pm 45] \ldots \ldots \ldots .160$

Figure 6.61 Transverse tip deflection at the right end, $w$, HSDT, $\Omega=10 \mathrm{rad} / \mathrm{s},[0 / \pm 45] \ldots \ldots \ldots .161$

Figure 6.62 Axial deflection at the right end, $u$, HSDT, $\Omega=10 \mathrm{rad} / \mathrm{s},[0 / \pm 45] \ldots \ldots \ldots \ldots \ldots \ldots \ldots . . . . . . . . . . . .162$

Figure 6.63 Transverse tip deflection at the left end, $w_{b}$, HSDT, $\Omega=20 \mathrm{rad} / \mathrm{s},[0 / \pm 45] \ldots \ldots \ldots . . .164$

Figure 6.64 Transverse tip deflection at the left end, $w_{s}$, HSDT, $\Omega=20 \mathrm{rad} / \mathrm{s},[0 / \pm 45] \ldots \ldots \ldots . .165$ 
Figure 6.65 Transverse tip deflection at the left end, $w$, HSDT, $\Omega=20 \mathrm{rad} / \mathrm{s},[0 / \pm 45] \ldots \ldots \ldots . . . .166$ Figure 6.66 Transverse tip deflection at the right end, $w_{b}, \mathrm{HSDT}, \Omega=20 \mathrm{rad} / \mathrm{s},[0 / \pm 45] \ldots \ldots \ldots .167$

Figure 6.67 Transverse tip deflection at the right end, $w_{s}$, HSDT, $\Omega=20 \mathrm{rad} / \mathrm{s},[0 / \pm 45] \ldots \ldots \ldots .168$

Figure 6.68 Transverse tip deflection at the right end, $w, \mathrm{HSDT}, \Omega=20 \mathrm{rad} / \mathrm{s},[0 / \pm 45] \ldots \ldots \ldots .169$

Figure 6.69 Axial deflection at the right end, $u$, HSDT, $\Omega=20 \mathrm{rad} / \mathrm{s},[0 / \pm 45] \ldots \ldots \ldots \ldots \ldots \ldots \ldots . . . . . . . . . .170$

Figure 6.70 Transverse tip deflection at the left end, $w_{b}, \mathrm{HSDT}, \Omega=10 \mathrm{rad} / \mathrm{s},[0 / \pm 20] \ldots \ldots \ldots . . .172$

Figure 6.71 Transverse tip deflection at the left end, $w_{s}$, HSDT, $\Omega=10 \mathrm{rad} / \mathrm{s},[0 / \pm 20] \ldots \ldots \ldots . .173$

Figure 6.72 Transverse tip deflection at the left end, $w$, HSDT, $\Omega=10 \mathrm{rad} / \mathrm{s},[0 / \pm 20] \ldots \ldots \ldots \ldots .174$

Figure 6.73 Transverse tip deflection at the right end, $w_{b}, \mathrm{HSDT}, \Omega=10 \mathrm{rad} / \mathrm{s},[0 / \pm 20] \ldots \ldots \ldots .175$

Figure 6.74 Transverse tip deflection at the right end, $w_{s}$, HSDT, $\Omega=10 \mathrm{rad} / \mathrm{s},[0 / \pm 20] \ldots \ldots \ldots .176$

Figure 6.75 Transverse tip deflection at the right end, $w, \mathrm{HSDT}, \Omega=10 \mathrm{rad} / \mathrm{s},[0 / \pm 20] \ldots \ldots \ldots . .177$

Figure 6.76 Axial deflection at the right end, $u$, HSDT, $\Omega=10 \mathrm{rad} / \mathrm{s},[0 / \pm 20] \ldots \ldots \ldots \ldots \ldots \ldots \ldots . . . . . . . . . .178$

Figure 6.77 Transverse tip deflection at the left end, $w_{b}$, HSDT, $\Omega=20 \mathrm{rad} / \mathrm{s},[0 / \pm 20] \ldots \ldots \ldots . .180$

Figure 6.78 Transverse tip deflection at the left end, $w_{s}$, HSDT, $\Omega=20 \mathrm{rad} / \mathrm{s},[0 / \pm 20] \ldots \ldots \ldots . . .181$

Figure 6.79 Transverse tip deflection at the left end, $w$, HSDT, $\Omega=20 \mathrm{rad} / \mathrm{s},[0 / \pm 20] \ldots \ldots \ldots . . . .182$

Figure 6.80 Transverse tip deflection at the right end, $w_{b}, \mathrm{HSDT}, \Omega=20 \mathrm{rad} / \mathrm{s},[0 / \pm 20] \ldots \ldots \ldots .183$

Figure 6.81 Transverse tip deflection at the right end, $w_{s}$, HSDT, $\Omega=20 \mathrm{rad} / \mathrm{s},[0 / \pm 20] \ldots \ldots \ldots .184$

Figure 6.82 Transverse tip deflection at the right end, $w, \mathrm{HSDT}, \Omega=20 \mathrm{rad} / \mathrm{s},[0 / \pm 20] \ldots \ldots \ldots .185$

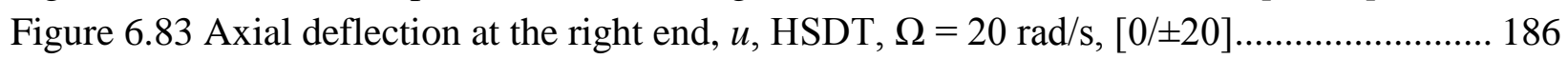

Figure 6.84 Transverse tip deflection at the left end, $w_{b}$, HSDT, $\Omega=20 \mathrm{rad} / \mathrm{s},[0 / \pm 45]_{\mathrm{s}} \ldots \ldots \ldots . .188$

Figure 6.85 Transverse tip deflection at the left end, $w_{s}$, HSDT, $\Omega=20 \mathrm{rad} / \mathrm{s},[0 / \pm 45]_{\mathrm{s}} \ldots \ldots \ldots . .189$

Figure 6.86 Transverse tip deflection at the left end, $w$, HSDT, $\Omega=20 \mathrm{rad} / \mathrm{s},[0 / \pm 45]_{\mathrm{s}} \ldots \ldots \ldots \ldots .190$

Figure 6.87 Transverse tip deflection at the right end, $w_{b}$, HSDT, $\Omega=20 \mathrm{rad} / \mathrm{s},[0 / \pm 45]_{\mathrm{s}} \ldots \ldots \ldots 191$

Figure 6.88 Transverse tip deflection at the right end, $w_{s}$, HSDT, $\Omega=20 \mathrm{rad} / \mathrm{s},[0 / \pm 45]_{\mathrm{s}} \ldots \ldots . . .192$

Figure 6.89 Transverse tip deflection at the right end, $w$, HSDT, $\Omega=20 \mathrm{rad} / \mathrm{s},[0 / \pm 45]_{\mathrm{s}} \ldots \ldots \ldots .193$

Figure 6.90 Axial deflection at the right end, $u$, HSDT, $\Omega=20 \mathrm{rad} / \mathrm{s},[0 / \pm 45]_{\mathrm{s}} \ldots \ldots \ldots \ldots \ldots \ldots \ldots . . . . . . . . .194$ 


\section{INTRODUCTION}

\subsection{Problem statement}

The study of the lateral vibration of a beam oscillating axially relative to fixed supports has applications in robot arms, motion of a support beam under earthquake induced oscillations, operation of a band saw and conveyor belts. The transverse vibrations of isotropic and composite beams undergoing a periodic axial motion relative to two fixed supports have been analyzed. Both these studies indicate that the amplitude of lateral vibration of an axially-moving beam is higher by about $40 \%$ than that of a beam without any axial motion. There are a few ways to reduce the amplitude of transverse vibration; one is to increase the stiffness of the beam, but this may increase the cost, add a weight penalty or both. The alternative proposed in the present research is to add piezoelectric layers which can be manipulated to attenuate the amplitude of the transverse vibration. The governing equations of lateral vibration of an axially-oscillating composite-material beam that incorporates the effect of piezoelectric layers are formulated using Hamilton's principle.

\subsection{Literature review}

\subsubsection{Isotropic moving beams}

Buffinton and Kane (1985) studied the response of a uniform beam moving longitudinally over the supports. Governing equations were formulated by assuming the supports as kinematic constraints on unrestrained beam. The beam was discretized using assumed-modes technique. The responses of the beam for different longitudinal motions of the beam were studied. 
Lee (1992) studied the response of beam moving over multiple supports. Hamilton's principle was used for the formulation. Assumed-modes technique was used to solve the equation of motion. Range-Kutta method was employed for numerical integration. The first flexural mode shape was used as the initial shape of the beam. The responses of the beam for different support conditions were studied.

Sreeram and Sivaneri (1997) investigated the lateral response of an isotropic beam oscillating longitudinally over fixed supports. A variational technique was used for formulating the governing equations. The $h-p$ version finite element formulation was developed. Convergence study was performed to find out the number of degree of freedom to produce accurate solution. Lagrange multiplier approach was used for applying the displacement constraints. The results were validated with Buffinton and Kane (1985) and Lee (1992). Time integration techniques such as central difference method, Houbolt's method, Wilson-theta method and Newmark's method were used for time integration and it wasconcluded that accurate results were produced using Wilson- theta method and Newmark's method.

\subsubsection{Laminated beams}

Reddy (2004) presented several theories for the analysis of laminated composite plates. Initially, the analysis was based on three dimensional elasticity theories. Later on equivalent single layer (ESL) theories were developed from three dimensional elasticity theories by making assumptions on the state of stress through the thickness. Classical laminate plate theory (CLPT) and first order shear deformation theories (FSDT) are the ESL theories. The CLPT ignores the effect of transverse shear deformation and may be inadequate for many cases. In FSDT, the transverse shear effects were included. The shear stress was assumed to be constant through the thickness. A shear correction factor was introduced to approximately account for the variation of 
shear stress through the thickness. Third order shear deformation theory was developed to avoid the approximation of shear stresses. Variational principles were used in obtaining the governing equations.

Singh, Rao, and Iyengar (1991) studied the response of unsymmetric laminated beams for large deflections using Von Karman's large deflection theory. Classical laminate plate theory, first order shear deformation theory and higher order shear deformation theories were used for formulating composite beams. The analysis was performed using elements having 8, 10, and 12 degrees of freedom per node. Direct-integration techniques were employed for time integration of equations for different boundary conditions, lay ups and slenderness ratios. Responses of isotropic and symmetric laminated beams were also investigated.

Marur and Kant (1998) modeled a beam with seven degrees of freedom per node for the transient analysis of composite and sandwich beams. Cubic interpolation of axial strain, linear interpolation transverse strain and quadratic interpolation of transverse shear strain were assumed. Each layer of the beam is assumed to be in the state of plane stress. A special lumping technique was used for the formation of diagonal mass matrix. The central difference method was used for time integration. Results for the higher order model were compared with the first order shear deformation model.

Lee and Lee (1990) investigated the vibrational characteristics of composite wings of various shapes. Shear deformation theory was used for the formulation. The effect of fiber orientation, aspect ratio, sweep angle and taper ratio of the composite wing on the vibrational characteristics was studied.

Kapania and Ranciti (1989) developed a one-dimensional beam element to perform nonlinear analysis on symmetric and unsymmetric laminated composite beams. The beam 
element has two nodes with ten degrees of freedom each. Displacements were interpolated using Hermite functions. It was observed that the in-plane boundary significantly affected the nonlinear vibrations. The effect of shear deformation was neglected for nonlinear vibrations and considered for linear vibrations. It was found that the large deflection theory neglecting the shear deformation produced reasonably accurate results for nonlinear analysis of thin beams.

Kapania and Singhvi (1991) studied the free-vibrational characteristics of rectangular and tapered laminated composite plates. The Rayleigh-Ritz method was used for the formulation of equations of motion. Chebyshev polynomials were used to interpolate the displacements. Numerical integration was performed using Gauss quadrature. Analysis was performed for isotropic, specially orthotropic, and symmetrically and unsymmetrically laminated plates.

Chandrasekaran (2000) studied the response of laminated composite beam oscillating axially on fixed supports. Classical laminate plate theory and first order shear deformation theories were used for the formulation of composite beams. Variational principle was used to derive the equations of motion. Lagrange multipliers were used to apply the displacement constraints. Newmark's time integration technique was used to find the response of the moving beam. The vibrational responses of symmetric and unsymmetric laminated beams were investigated.

Kadivar and Mohebpour (1998) studied the response of unsymmetric laminated composite beams subjected to moving load. Classical laminate plate theory, first-order shear deformation theory and third-order shear deformation theories were used for the formulation of composite beam. Hamilton's principle was used for deriving the governing equations. Time integration was performed using Newmark's method. Dynamic responses of symmetric and unsymmetric cross ply laminates were also studied. 
Sivaneri and Nagappan and (2012) investigated the response of laminated composite moving beam based on higher-order shear deformation theory. Displacement boundary conditions were applied using Lagrange multiplier approach. Hamilton's principle was used to derive the equations of motion. Newmark's time integration method was used to find the response of the beam. Responses of symmetric and unsymmetric laminated moving beams were studied and compared with first order shear deformation model.

\subsubsection{Piezoelectricity}

Bailey and Hubbard (1985) designed an active vibrational damper to attach to a cantilevered beam using distributed piezoelectric actuators. The voltage supplied to the damper was controlled using Lyapunov control technique. The response was compared with linear control-gain and constant-amplitude control models.

Lee (1988) developed an analytical model for the analysis of laminated piezoelectric plates for sensing its stretching, bending, torsion and shearing and controlling its vibrations. Formulation for sensors and actuators were presented. Reciprocal relation between sensors and actuators were presented.

Koconis (1993) developed an analytical model to investigate the response of laminated beams, plates, and shells embedded with piezoelectric actuators due to an applied voltage. Formulation was done using a linear, shallow shell theory. Shear deformation effects were included in the formulation. The Ritz method was used to formulate the equations of motion. Analytical results were compared with experimental results.

Mitchell and Reddy (1994) proposed a hybrid theory for the analysis of laminated composite plates embedded with piezoelectric laminates. Third-order shear deformation theory was used to model the displacements field. The electric potential was discretized along the 
thickness direction. The governing equations were derived using Hamilton's principle. The theory developed here was applied to laminates with thick piezoelectric layers and multi layered piezoelectric laminates.

Saravanos, Heyliger, and Hopkins (1995) presented the mechanics for the analysis of laminated composite plates with integrated sensors and actuators. First-order shear deformation theory was used to represent the displacement variation. Electric potential was assumed to vary linearly through the thickness. Hamilton's principle was used to formulate the governing equation. Formulations were developed for quasi-static and dynamic responses of thin and thick piezoelectric laminates. Static and free vibrational analyses were also performed.

Donthireddy and Chandrashekhara (1996) developed a mathematical model based on layerwise plate theory for laminated composite beams with piezoelectric actuators. Voltages were supplied to the actuators. Response of the laminated beams due to supplied voltage was studied. The influence of fiber orientations and boundary conditions on the response of the beams was studied.

Reddy (1997) presented the theoretical formulations, Navier solutions and finite element models for laminated composite plates with integrated sensors and actuators based on classical laminated plate theory and shear deformation theories. A negative velocity feedback control that couples the sensors and actuators to actively control the dynamic response of the structure was described.

Balamurugan and Narayanan (2001) studied the active vibration control of a cantilevered beam integrated with piezoelectric sensors and actuators. Euler-Bernoulli beam theory was used for the formulation of beam. Classical control techniques, direct proportional feedback, constantgain negative-velocity feedback, Lyapunov feedback, optimal control technique, and linear 
quadratic regulator (LQR) were presented. The controlled response of a beam for different types of loading was studied.

Narayanan and Balamurugan (2002) proposed an analytical model for laminated composite structures embedded with piezoelectric layers using beam, plate, and shell elements. Pyroelectric effect was considered in addition to the piezoelectric effect during the formulation of governing equations. Several feedback controls; Constant-gain negative-velocity feedback, Lyapunov feedback and linear quadratic regulator were presented for active vibration control and concluded that LQR was effective among the three. Applications of the smart structures were discussed.

Liewet al. (2004) studied the response of laminated composite plates integrated with piezoelectric sensor and actuator patches using element-free Galerkin's method. First -order shear deformation theory was used to model the displacement fields. Variational principle was used for the governing equations. A simple control technique including both displacement and velocity feedback control was used for active vibration control. The influence of fiber orientation, location of sensor, and actuator patches on the vibration characteristics was studied.

Moita et al. (2004) presented a finite element formulation of thin laminated structures embedded with sensors and actuators for active vibration control. Kirchhoff's classical laminate plate theory was used for the formulation of composite plates. The structure was discretized using triangular plate or shell elements having 18 displacement degrees of freedom and one electric potential degree of freedom. Negative-velocity feedback was used for active vibration control. Newmark's method was used for time integration.

\subsection{Need for present research}


Several researchers have studied active vibration control using piezoelectricity for nonmoving plates and beams. Due to wide range of applications of the moving beams in the areas of robotics, flexible manipulators, etc., it is necessary to study the active vibration control of laminated composite moving beams. Present research focusses on reducing the vibration response of an axially oscillating beam over fixed supports using piezoelectric active vibration control.

\subsection{Objectives}

Objectives of this thesis are:

- To formulate the problem of lateral vibration of an axially-oscillating composite-material beam using Hamilton's principle that incorporates the presence and effect of piezoelectric layers.

- To develop a numerical model based on the finite element method and incorporate the model in a MATLAB program.

- To investigate the role of piezoelectric sensors and actuators in reducing the excessive vibrational amplitude due to axial motion of the beam.

- To conduct a parametric study by varying the frequency of axial motion and by considering several symmetric and unsymmetric lay-ups.

\subsection{Organization of the thesis}

- Chapter one includes the problem statement, information on the previous work, and objectives of the thesis.

- Chapter two discusses briefly to the nature and function of piezoelectric materials. 
- Chapter three discusses the theoretical formulation to reduce the plate theory to the beam theory and the variational principle applied.

- Chapter four deals with the finite element formulation to derive the equations of motion for the discretized beam.

- Chapter five discuss the numerical methods used in the time domain as applied to the present research.

- Chapter six presents the results and the associated discussions.

- Chpater seven includes the conclusions and future recommendations. 


\section{PIEZOELECTRIC MATERIALS}

The inherent material damping present may not be sufficient to reduce the amplitude of undesirable vibrations. An external source of vibration control is necessary in such a case. The devices that reduce the vibrational amplitude are classified into passive and active vibration control techniques. Passive control is based on energy absorption or dissipation principles and uses friction dampers, shock absorbers, and viscoelastic dampers, etc. In active vibration control, a counteracting force or moment is applied to suppress the vibration. External power sources such as electrical or pneumatic systems are used to provide the counteracting forces. The selfadaptivity property of this technique generates variable control forces or moments for different operating conditions. This chapter provides a brief introduction to the piezoelectric materials.

\subsection{Dielectrics}

Dielectric materials, made of crystals, when subjected to an electric field undergo deformation due to the movement of positive and negative charges within a crystal. The dielectric crystal lattice is assumed to be made up of cations and anions, bonded by springs. On application of an electric field the cations are displaced along the applied electric field and anions opposite to the electric field leading to the deformation of the material. Dielectrics are classified into two types namely, non-polar and polar.

In non-polar dielectrics, the centers of positive and negative charges coincide inside the atoms and do not possess dipole moment. When an electric field is applied onto these crystals, the centers of positive and negative charges displace and dipole moment is induced. On removal of the electric field, the induced dipole moments disappear. In polar dielectrics, the centers of 
positive and negative charges are separated by a distance and they possess a dipole moment. When an electric field is applied onto these crystals, the electric dipoles tend to orient themselves along the electric field.

\subsubsection{Piezoelectric materials}

Polar dielectric crystals are classified into centrosymmetric (having a center of symmetry) and non centrosymmetric (without a center of symmetry) crystals as shown in Figure 2.1. The cations and anions are assumed to be connected by springs in a crystal. When an electric field is applied onto centrosymmetric crystals, the expansion and contraction caused by the symmetric movement of cations and anions are cancelled between the neighboring springs, resulting in zero net displacement. But, in real case, aharmonicity exists between the chemical bonds resulting in a small deformation of the crystal. The strain calculated in this case is proportional to the square of the applied electric field and is independent of the direction of the electric field. This is known as the electrostrictive effect. As aharmonicity of bonds prevails in all dielectrics, they are in general, electrostrictive.

non-centrosymmetric crystals, when subjected to an electric field, exhibit a significant deformation due to the asymmetric movement of the ions. These crystals also exhibit electrostrictive effect due to the aharmonicity of bonds, which is small when compared to the asymmetric displacement. These materials are called piezoelectric materials. The strains calculated are directly proportional to the applied electric field. The strain in piezoelectric materials is extensive or compressive depending on the polarity of the field. This is known as "indirect" piezoelectric effect as shown in Figure 2.2. Piezoelectric materials also exhibit a "direct" piezoelectric effect, which means that when the material is subjected to an external 
strain by application of the stress/pressure, positive and negative charges are deposited on opposite faces resulting in an electric field across the crystal as shown in Figure 2.3. The direct piezoelectric effect is used in sensors to measure the structural response and inverse piezoelectric effect is used in actuators for vibration control.

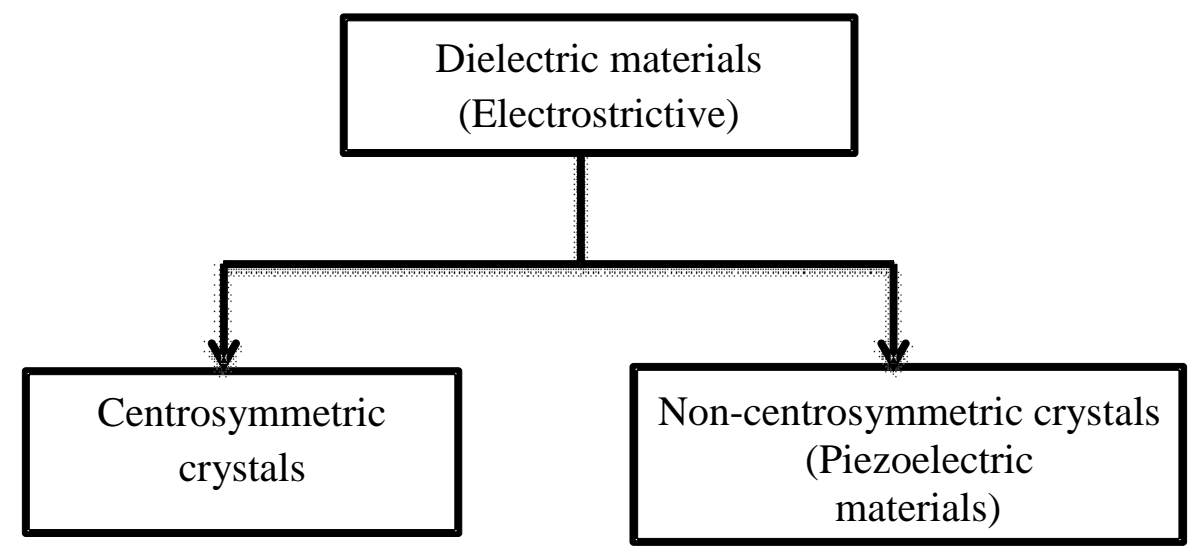

Figure 2.1 Classification of dielectric materials

Natural and synthetic piezoelectric materials exhibiting piezoelectric effect are (Dökmeci, 1983):

1. Natural crystals: quartz, rochelle salt, and ammonium phosphate

2. Liquid crystals

3. Noncrystalline materials: rubber, paraffin, glass

4. Textures: wood and bone

5. Synthetic piezoelectric materials:

a. Piezoceramics: lead zirconate titanate (PZT), barium titanate, lead niobate, and lead lanthanum zirconate titanate (PLZT)

b. Crystallines: ammonium dihydrogen phosphate and lithium sulfate

c. Piezoelectric polymer: polyvinylidene fluoride $\left(\mathrm{PVDF}\right.$ or $\left.\mathrm{PVF}_{2}\right)$ 
Piezoelectric layers attached to a structure should be flexible and not brittle, so that they do not break during high vibrations. Also, they should not alter the dynamic characteristics of the structure much. For these reasons, piezoelectric layers made of PZT or PVDT are extensively used as sensors and actuators. Also the thickness of the piezoelectric layers should be small enough such that the strains are taken as the outer surface strains of the structure and assumed constant through the thickness of piezoelectric layer.

Piezoelectric ceramic materials exhibit centrosymmetric structure above certain temperature and non centrosymmetric structure below this temperature. This temperature is called the Curie temperature. Below the Curie temperature, the ceramics have randomly oriented dipoles and the net dipole moment is zero. The material is isotropic and does not exhibit piezoelectric effect. The material becomes piezoelectric by polarization. In this process a strong electric field is applied to the material and the dipoles tend to orient parallel to the electric field. The dipoles maintain this orientation even after the electric field is removed. This is called remnant polarization.

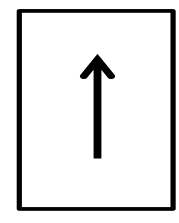

(a) Polarized piezoelectric material

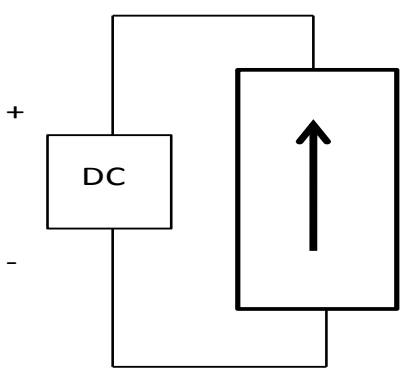

(b) Material is stretched when a positive DC voltage is applied

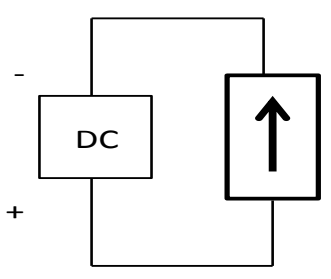

(c) Material is compressed when DC voltage with reverse polarity is applied.

Figure 2.2 Indirect piezoelectric effect 


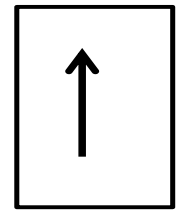

(a) Poled piezoelectric material

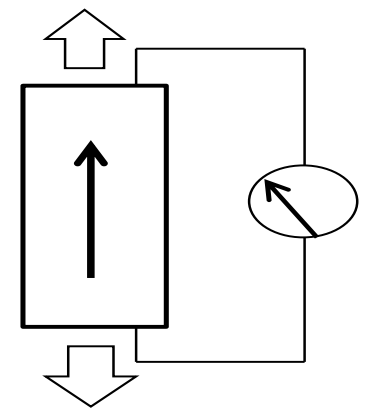

(b) Negative voltage is generated due to tensile stress.

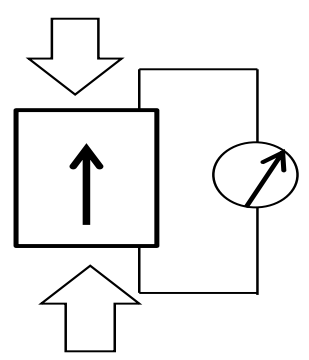

(c) Positive voltage is generated due to compressive stress.

Figure 2.3 Direct piezoelectric effect material 


\section{THEORETICAL FORMULATION}

\subsection{Introduction}

Active vibration control using piezoelectric materials is considered in this research. Sensors or transducers are attached to the structure to measure its dynamic response and the actuators are attached to suppress the level of vibrations. A structure with active vibration control is known as a smart structure.

Composite materials are widely used in engineering applications because of their high strength to weight ratio, corrosion resistance, high fatigue life, etc. Owing to their wide range of applications, different plate theories are developed for the analysis of composite plates. Composite beams are used as structural members where in they are subjected to axial, transverse, and torsional loadings. The formulation of moving composite beams with added piezoelectric layers is carried out in this chapter; a systematic reduction of different plate theories for beam adaptation is also discussed.

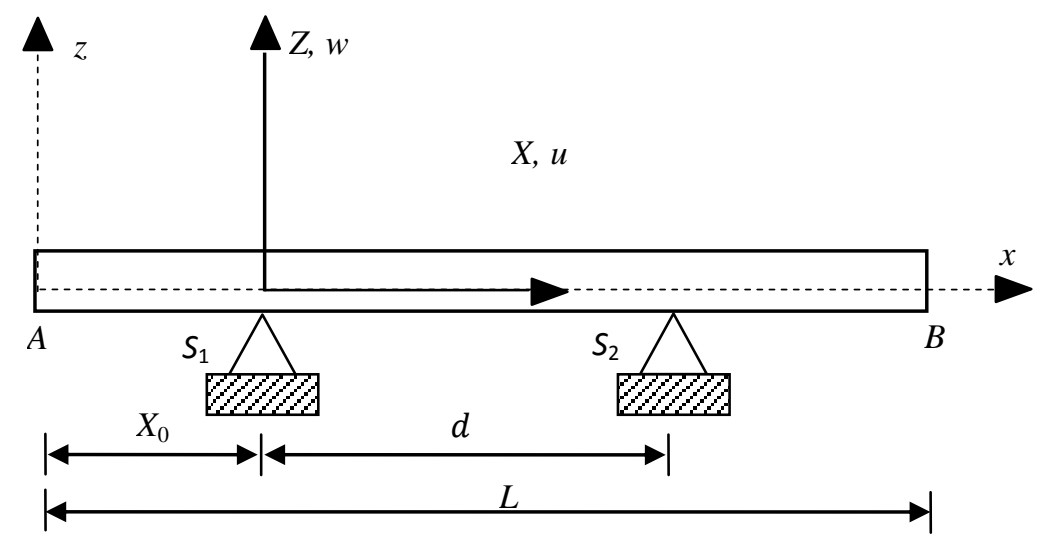

Figure 3.1 Coordinate systems for the moving beam 


\subsection{Moving Beam Coordinate Systems}

Consider a beam $A B$ (Figure 3.1 of length $L$ is oscillating longitudinally relative to the fixed supports $S_{1}$ and $S_{2}$, separated by a distance $d$. An inertial frame $(X, Z)$ is defined at support $S_{1}$. The initial distance between the left end of the beam and the support $S_{1}$ is given by $X_{0}$. The horizontal movement of the beam is represented by $X_{A}(t)$ which is always positive. A moving frame $(x, z)$ is defined at the left end of the beam. The transformation between the inertial and moving frames is given by

$$
\begin{gathered}
x(t)=X(t)-X_{A}(t) \\
z(t)=Z(t)
\end{gathered}
$$

The beam is capable of deforming in the longitudinal $(u)$ and the transverse $(w)$ directions. The supports move in the moving frame with respect to the left end of the beam.

The relative motion of the supports at any time is given by

$$
\begin{gathered}
x_{S_{1}}(t)=-X_{A}(t) \\
x_{S_{2}}(t)=-X_{A}(t)+d
\end{gathered}
$$

\subsection{Beam Motion}

The longitudinal motion imparted to the beam is similar to the one assumed by Sreeram and Sivaneri (1997), Chandrasekaran (2000) and Sivaneri and Nagappan (2013). It is given as

$$
X_{A}(t)=-X_{0}+A \sin (\Omega t)
$$

where $A$ is the amplitude and $\Omega$ the frequency of the longitudinal rigid-body motion of the beam. Then the relative motion of the supports in moving coordinates, Eq. (3.2), becomes

$$
\begin{gathered}
x_{S_{1}}(t)=X_{0}-A \sin (\Omega t) \\
x_{S_{2}}(t)=X_{0}-A \sin (\Omega t)+d
\end{gathered}
$$


The longitudinal rigid-body velocity and acceleration of the beam are

$$
\begin{array}{r}
v_{B}^{L}=A \Omega \cos (\Omega t) \\
a_{B}^{L}=-A \Omega^{2} \sin (\Omega t)
\end{array}
$$

\subsection{Composite Plate Theories}

The three prominent laminate plate theories considered in this research are the classical laminate plate theory (CLPT), first-order shear deformation theory (FSDT) and higher-order shear deformation theory (HSDT). For each theory, the systematic reduction to beams is outlined.

\subsubsection{Classical Laminate Plate theory}

The Classical laminate plate theory is one of the equivalent single layer theories. It is assumed that Kirchoff's hypothesis holds in CLPT, stated as below

1. Straight lines perpendicular to mid surface (transverse normal) remain perpendicular after the deformation $\left(\gamma_{x z}=0\right.$ and $\left.\gamma_{y z}=0\right)$

2. The transverse normal remains straight after deformation.

3. The transverse normal is inextensible. $\left(\varepsilon_{z}=0\right)$

The last two assumptions indicate that the transverse displacement $(w)$ is independent of the transverse coordinate $(z)$.

In addition to the Kirchhoff's hypothesis, the following are assumed for a laminated composite plate:

4. Layers are perfectly bonded.

5. The material is linearly elastic and has two planes of material symmetry.

6. The layers have uniform thickness.

7. The transverse shear stresses on the top and bottom surfaces of the laminate are zero. 
8. The strains and displacements are small with moderate rotations.

The inplane and the lateral displacements of a composite plate in the coordinate directions indicated in Fig. 3.2, are represented as

$$
\begin{gathered}
u(x, y, z, t)=u_{o}(x, y, t)-z \frac{\partial w_{o}}{\partial x} \\
v(x, y, z, t)=v_{o}(x, y, t)-z \frac{\partial w_{o}}{\partial y} \\
w(x, y, z, t)=w_{o}(x, y, t)
\end{gathered}
$$

where $u_{o}, v_{o}$, and $w_{o}$ are the midplane displacements and $w_{o}$ is made up of only bending component as the transverse shear deformation is neglected.

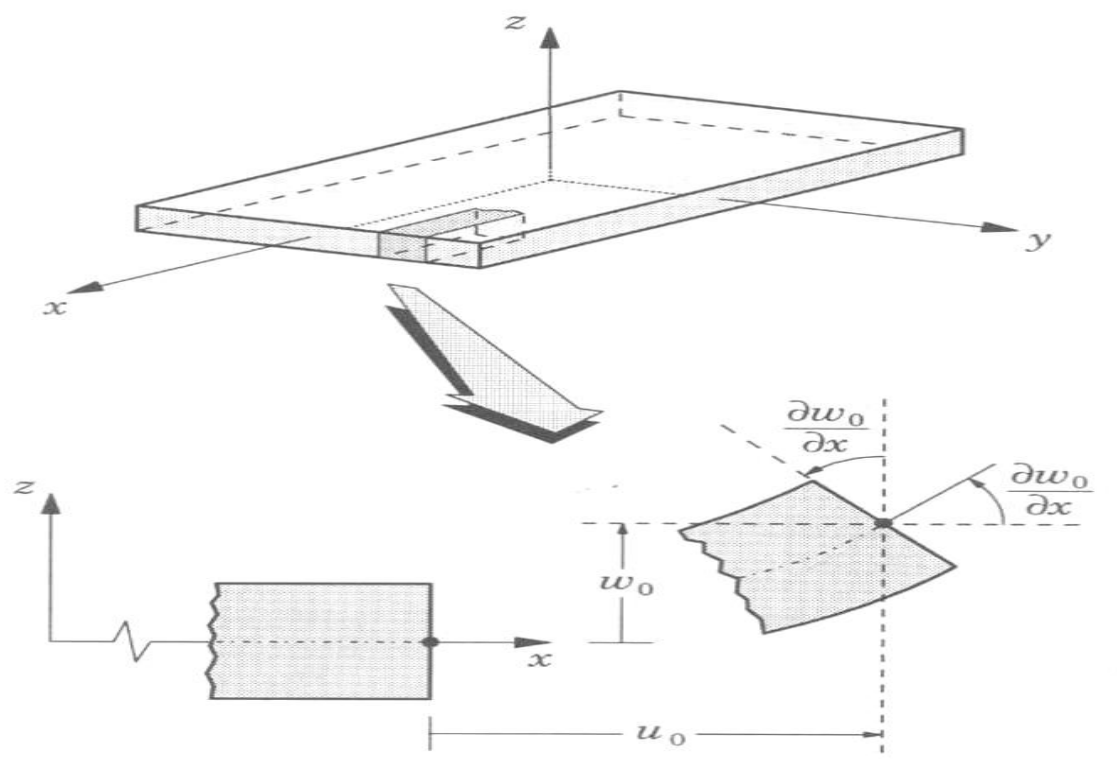

Figure 3.2 Undeformed and deformed geometries of an edge of a plate under Kirchhoff's assumption for CLPT [Reddy (1997)]

The strains associated with the above displacement field are

$$
\left\{\begin{array}{c}
\varepsilon_{\mathrm{x}} \\
\varepsilon_{\mathrm{y}} \\
\gamma_{\mathrm{xy}}
\end{array}\right\}=\left\{\begin{array}{c}
\varepsilon_{\mathrm{x}}^{(0)} \\
\varepsilon_{\mathrm{y}}^{(0)} \\
\gamma_{\mathrm{xy}}^{(0)}
\end{array}\right\}+\mathrm{z}\left\{\begin{array}{c}
\varepsilon_{\mathrm{x}}^{(1)} \\
\varepsilon_{\mathrm{y}}^{(1)} \\
\gamma_{\mathrm{xy}}^{(1)}
\end{array}\right\}
$$


where,

$$
\begin{gathered}
\left\{\begin{array}{c}
\varepsilon_{\mathrm{x}}^{(0)} \\
\varepsilon_{\mathrm{y}}^{(0)} \\
\gamma_{\mathrm{xy}}^{(0)}
\end{array}\right\}=\left\{\begin{array}{c}
\frac{\partial u_{o}}{\partial x}+\frac{1}{2}\left(\frac{\partial w}{\partial x}\right)^{2} \\
\frac{\partial v_{o}}{\partial y}+\frac{1}{2}\left(\frac{\partial w}{\partial y}\right)^{2} \\
\frac{\partial u_{o}}{\partial y}+\frac{\partial v_{o}}{\partial x}+\frac{\partial w}{\partial x} \frac{\partial w}{\partial y}
\end{array}\right\} \\
\left\{\begin{array}{l}
\varepsilon_{\mathrm{x}}^{(1)} \\
\varepsilon_{\mathrm{y}}^{(1)} \\
\gamma_{\mathrm{xy}}^{(1)}
\end{array}\right\}=\left\{\begin{array}{c}
-\frac{\partial^{2} w}{\partial x^{2}} \\
-\frac{\partial^{2} w}{\partial y^{2}} \\
-2 \frac{\partial^{2} w}{\partial x \partial y}
\end{array}\right\}
\end{gathered}
$$

The $\left\{\epsilon^{(0)}\right\}$ are called membrane (axial) or midplane strains and $\left\{\epsilon^{(1)}\right\}$ are called flexural (bending) strains. These strains are also called as the Von-kármán strains and the plate theory associated with these is called as Von-kármán plate theory.

\section{$\underline{\text { CLPT Constitutive equations with Piezoelectric Layers }}$}

The lamina constitutive equations of a $k^{\text {th }}$ piezoelectric lamina coupling the elastic, electric, and piezoelectric field given in the material coordinate system (defined as $x_{1}, x_{2}, x_{3}$ ) are

$$
\begin{gathered}
\left\{\begin{array}{l}
\sigma_{1} \\
\sigma_{2} \\
\sigma_{6}
\end{array}\right\}^{k}=\left[\begin{array}{lll}
Q_{11} & Q_{12} & Q_{16} \\
Q_{12} & Q_{22} & Q_{26} \\
Q_{16} & Q_{26} & Q_{66}
\end{array}\right]^{k}\left\{\begin{array}{l}
\varepsilon_{1} \\
\varepsilon_{2} \\
\gamma_{6}
\end{array}\right\}^{k}-\left[\begin{array}{ccc}
0 & 0 & e_{31} \\
0 & 0 & e_{32} \\
0 & 0 & 0
\end{array}\right]^{k}\left\{\begin{array}{l}
E_{1} \\
\left.E_{2}\right\} \\
E_{3}
\end{array}\right\}^{k} \\
\left\{\begin{array}{l}
D_{1} \\
D_{2} \\
D_{3}
\end{array}\right\}^{k}=\left[\begin{array}{ccc}
0 & 0 & 0 \\
0 & 0 & 0 \\
e_{31} & e_{32} & 0
\end{array}\right]^{k}\left\{\begin{array}{l}
\varepsilon_{1} \\
\varepsilon_{2} \\
\gamma_{6}
\end{array}\right\}^{k}+\left[\begin{array}{ccc}
\epsilon_{11} & 0 & 0 \\
0 & \epsilon_{22} & 0 \\
0 & 0 & \epsilon_{33}
\end{array}\right]^{k}\left\{\begin{array}{l}
E_{1} \\
E_{2} \\
E_{3}
\end{array}\right\}^{k}
\end{gathered}
$$

where $\sigma_{i}, \varepsilon_{\mathrm{i}}, D_{i}$, and $E_{i}$ are the stress, strain, dielectric displacement, and the applied electric field, respectively; $Q_{i j}$ are the material stiffness matrix components; $e_{\mathrm{ij}}$ are the piezoelectric moduli; and $\epsilon_{\mathrm{ij}}$ are the dielectric permittivity for the $k^{\text {th }}$ lamina in material coordinate system. The electric 
field is given in terms of voltage $(\varphi)$ and thickness of the layer $(t)$ as $E=-\frac{\varphi}{t}$. For a nonpiezoelectric layer the terms corresponding to the electric field disappear.

The piezoelectric moduli can expressed in terms of the material stiffness matrix, $[Q]$, and dielectric constant matrix, $[d]$ as

$$
[e]=[Q][d]^{T}
$$

or

$$
[e]^{T}=[d][Q]
$$

On transformation to the laminate coordinate system, $(x, y, z)$

$$
\begin{gathered}
\left\{\begin{array}{c}
\sigma_{x} \\
\sigma_{y} \\
\tau_{x y}
\end{array}\right\}^{k}=\left[\begin{array}{lll}
\bar{Q}_{11} & \bar{Q}_{12} & \bar{Q}_{16} \\
\bar{Q}_{12} & \bar{Q}_{22} & \bar{Q}_{26} \\
\bar{Q}_{16} & \bar{Q}_{26} & \bar{Q}_{66}
\end{array}\right]^{k}\left\{\begin{array}{c}
\varepsilon_{x} \\
\varepsilon_{y} \\
\gamma_{x y}
\end{array}\right\}^{k}-\left[\begin{array}{lll}
0 & 0 & \overline{\mathrm{e}}_{31} \\
0 & 0 & \overline{\mathrm{e}}_{32} \\
0 & 0 & \overline{\mathrm{e}}_{36}
\end{array}\right]^{k}\left\{\begin{array}{l}
E_{x} \\
E_{y} \\
E_{z}
\end{array}\right\} \\
\left\{\begin{array}{l}
D_{x} \\
D_{y} \\
D_{z}
\end{array}\right\}=\left[\begin{array}{ccc}
0 & 0 & 0 \\
0 & 0 & 0 \\
\overline{\mathrm{e}}_{31} & \overline{\mathrm{e}}_{32} & \overline{\mathrm{e}}_{36}
\end{array}\right]^{k}\left\{\begin{array}{c}
\varepsilon_{x} \\
\varepsilon_{y} \\
\gamma_{x y}
\end{array}\right\}^{k}+\left[\begin{array}{ccc}
\bar{\epsilon}_{11} & 0 & 0 \\
0 & \bar{\epsilon}_{22} & 0 \\
0 & 0 & \bar{\epsilon}_{33}
\end{array}\right]^{k}\left\{\begin{array}{l}
E_{x} \\
E_{y} \\
E_{z}
\end{array}\right\}
\end{gathered}
$$

$\bar{Q}_{i j}$ are the stiffness matrix components in laminate coordinate system; $\bar{e}_{\mathrm{ij}}$ are the piezoelectric moduli; and $\bar{\epsilon}_{\mathrm{ij}}$ are the dielectric permittivity for the $k^{\text {th }}$ lamina in laminate coordinate system. The inplane force resultants are defined as

$$
\left\{\begin{array}{c}
N_{x} \\
N_{y} \\
N_{x y}
\end{array}\right\}=\int_{-h / 2}^{h / 2}\left\{\begin{array}{c}
\sigma_{x} \\
\sigma_{y} \\
\tau_{x y}
\end{array}\right\} d z
$$

Where $h$ is the thickness of the laminate.

These are the forces acting per unit length at the mid plane. Similarly the moment resultants are defined as 


$$
\left\{\begin{array}{l}
M_{x} \\
M_{y} \\
M_{z}
\end{array}\right\}=\int_{-h / 2}^{h / 2}\left\{\begin{array}{c}
\sigma_{x} \\
\sigma_{y} \\
\tau_{x y}
\end{array}\right\} z d z
$$

The stress resultants form a system that is quasistatically equivalent to the stress system on a laminate, but are applied at the geometric mid plane. The laminate constitutive equations in terms of force and moment resultants are

$$
\begin{aligned}
& \left\{\begin{array}{c}
N_{x} \\
N_{y} \\
N_{x y}
\end{array}\right\}=\left[\begin{array}{lll}
A_{11} & A_{12} & A_{16} \\
A_{12} & A_{22} & A_{26} \\
A_{16} & A_{26} & A_{66}
\end{array}\right]\left\{\begin{array}{c}
\varepsilon_{\mathrm{x}}^{(0)} \\
\varepsilon_{\mathrm{y}}^{(0)} \\
\gamma_{\mathrm{xy}}^{(0)}
\end{array}\right\}+\left[\begin{array}{lll}
B_{11} & B_{12} & B_{16} \\
B_{12} & B_{22} & B_{26} \\
B_{16} & B_{26} & B_{66}
\end{array}\right]\left\{\begin{array}{c}
\varepsilon_{\mathrm{x}}^{(1)} \\
\varepsilon_{\mathrm{y}}^{(1)} \\
\gamma_{\mathrm{xy}}^{(1)}
\end{array}\right\}-\left\{\begin{array}{l}
N_{x}^{P} \\
N_{y}^{P} \\
N_{x y}^{P}
\end{array}\right\} \\
& \left\{\begin{array}{c}
M_{x} \\
M_{y} \\
M_{x y}
\end{array}\right\}=\left[\begin{array}{lll}
B_{11} & B_{12} & B_{16} \\
B_{12} & B_{22} & B_{26} \\
B_{16} & B_{26} & B_{66}
\end{array}\right]\left\{\begin{array}{l}
\varepsilon_{\mathrm{x}}^{(0)} \\
\varepsilon_{\mathrm{y}}^{(0)} \\
\gamma_{\mathrm{xy}}^{(0)}
\end{array}\right\}+\left[\begin{array}{lll}
D_{11} & D_{12} & D_{16} \\
D_{12} & D_{22} & D_{26} \\
D_{16} & D_{26} & D_{66}
\end{array}\right]\left\{\begin{array}{l}
\varepsilon_{\mathrm{x}}^{(1)} \\
\varepsilon_{\mathrm{y}}^{(1)} \\
\gamma_{\mathrm{xy}}^{(1)}
\end{array}\right\}-\left\{\begin{array}{l}
M_{x}^{P} \\
M_{y}^{P} \\
M_{x y}^{P}
\end{array}\right\}
\end{aligned}
$$

where $[A]$ is the extension stiffness matrix, $[B]$ is the bending-extension coupling matrix and $[D]$ is the bending stiffness matrix; $N^{P}$ and $M^{P}$ are the piezoelectric stress resultants. In terms of lamina properties, the quantities in Eq. (3.15) are written as 


$$
\begin{gathered}
A_{i j}=\sum_{k=1}^{n}\left(\bar{Q}_{i j}\right)_{k} t_{k} \\
B_{i j}=\sum_{k=1}^{n}\left(\bar{Q}_{i j}\right)_{k} t_{k} \bar{z}_{k} \\
D_{i j}=\sum_{k=1}^{n}\left(\bar{Q}_{i j}\right)_{k}\left(t_{k} \bar{z}_{k}^{2}-\frac{t_{k}{ }^{3}}{12}\right) \\
\left\{N^{P}\right\}=\sum_{k=1}^{n}(\bar{Q})_{k} t_{k}[d]_{k}^{T}\{E\}_{k}=-\sum_{k=1}^{n}(\bar{Q})_{k}[d]_{k}^{T}\{\varphi\}_{k} \\
\left\{M^{P}\right\}=\sum_{k=1}^{n}(\bar{Q})_{k} t_{k} \bar{Z}_{k}[d]_{k}^{T}\{E\}_{k}=-\sum_{k=1}^{n}(\bar{Q})_{k} \bar{Z}_{k}[d]_{k}^{T}\{\varphi\}_{k}
\end{gathered}
$$

where $t_{k}$ is the thickness of the $k^{\text {th }}$ layer and $\bar{z}_{k}$ is the distance from mid plane to the middle of the $k^{\text {th }}$ layer, and $n$ is the total number of layers.

\subsubsection{First-order shear deformation theory:}

The first order shear deformation theory is an improvement over the CLPT. The CLPT neglects the transverse shear strain, which may lead to quantifiable errors in composite plates and beams due to the low shear modulus of composites. In FSDT the perpendicularity of the transverse normal to the midplane is relaxed and as a result shear strains are included in the formulation. The displacement field can be expressed as

$$
\begin{aligned}
& u=u_{o}+z \phi_{x} \\
& v=v_{o}+z \phi_{y} \\
& w=w_{b}+w_{s}
\end{aligned}
$$

where $u_{o}$ and $v_{o}$ are the in-plane displacements of the mid plane; $w_{b}$ and $w_{s}$ are the bending and shear components of the transverse displacement; and $\phi_{x}$ and $\phi_{y}$ are the rotations of the transverse normal with respect to the $y$ and $x$ axes respectively as shown in Fig. (3.3). 


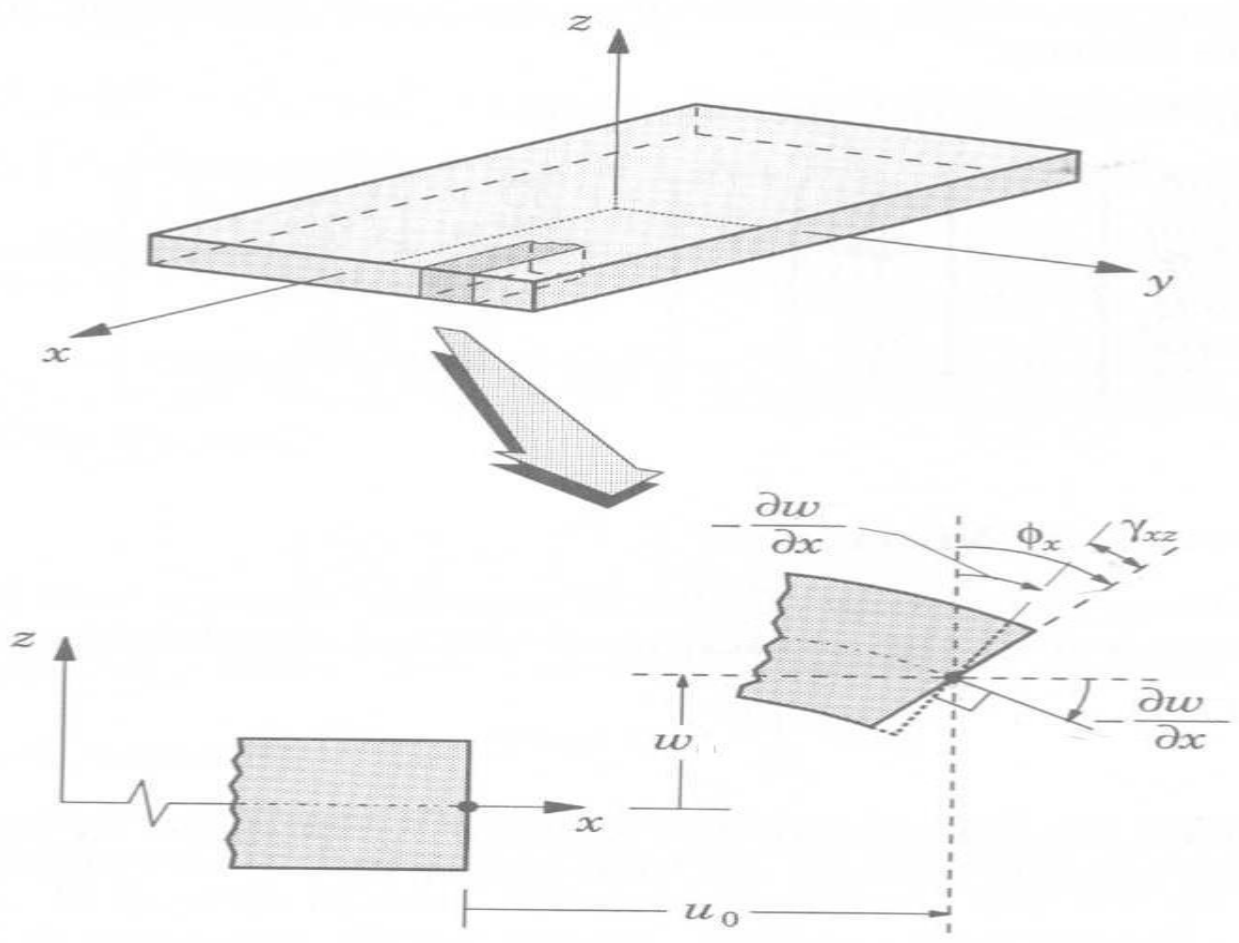

Figure 3.3 Undeformed and deformed geometries of an edge of a plate under the assumptions of first-order plate theory [Reddy (1997)]

From Figure 3.3

$$
\begin{aligned}
& \phi_{x}=-\frac{\partial w}{\partial x}+\gamma_{x z} \\
& \phi_{y}=-\frac{\partial w}{\partial y}+\gamma_{y z}
\end{aligned}
$$

where $\gamma_{x z}$ and $\gamma_{y z}$ are the transverse shear strains due to the relaxation of perpendicularity of the transverse normal and are expressed as $\partial w_{s} / \partial x$ and $\partial w_{s} / \partial y$ respectively. Thus

$$
\begin{aligned}
& \phi_{x}=-\frac{\partial w}{\partial x}+\frac{\partial w_{s}}{\partial x}=-\frac{\partial w_{b}}{\partial x} \\
& \phi_{y}=-\frac{\partial w}{\partial y}+\frac{\partial w_{s}}{\partial y}=-\frac{\partial w_{b}}{\partial y}
\end{aligned}
$$

The strains associated with the above displacement field are 


$$
\left\{\begin{array}{c}
\varepsilon_{\mathrm{x}} \\
\varepsilon_{\mathrm{y}} \\
\gamma_{\mathrm{xy}}
\end{array}\right\}=\left\{\begin{array}{c}
\varepsilon_{\mathrm{x}}^{(0)} \\
\varepsilon_{\mathrm{y}}^{(0)} \\
\gamma_{\mathrm{xy}}^{(0)}
\end{array}\right\} \begin{array}{r}
\varepsilon_{\mathrm{x}}^{(1)} \\
+\mathrm{z} \varepsilon_{\mathrm{y}}^{(1)} \\
\gamma_{\mathrm{xy}}^{(1)}
\end{array}
$$

where,

$$
\begin{gathered}
\left\{\begin{array}{c}
\varepsilon_{\mathrm{x}}^{(0)} \\
\varepsilon_{\mathrm{y}}^{(0)} \\
\gamma_{\mathrm{xy}}^{(0)}
\end{array}\right\}=\left\{\begin{array}{l}
\frac{\partial u_{o}}{\partial x}+\frac{1}{2}\left(\frac{\partial w_{b}}{\partial x}+\frac{\partial w_{s}}{\partial x}\right)^{2} \\
\frac{\partial v_{o}}{\partial y}+\frac{1}{2}\left(\frac{\partial w_{b}}{\partial y}+\frac{\partial w_{s}}{\partial y}\right)^{2} \\
\frac{\partial u_{o}}{\partial y}+\frac{\partial v_{o}}{\partial x}+\frac{\partial w}{\partial x} \frac{\partial w}{\partial y}
\end{array}\right\} \\
\left\{\begin{array}{l}
\varepsilon_{\mathrm{x}}^{(1)} \\
\varepsilon_{\mathrm{y}}^{(1)} \\
\gamma_{\mathrm{xy}}^{(1)}
\end{array}\right\}=\left\{\begin{array}{c}
-\frac{\partial^{2} w_{b}}{\partial x^{2}} \\
-\frac{\partial^{2} w_{b}}{\partial y^{2}} \\
-2 \frac{\partial^{2} w_{b}}{\partial x \partial y}
\end{array}\right\}
\end{gathered}
$$

\section{FSDT Constitutive equations with Piezoelectric Layers}

The lamina constitutive equations of the $k^{\text {th }}$ piezoelectric lamina coupling the elastic, electric, and piezoelectric field in material coordinate system (defined as $x_{1}, x_{2}, x_{3}$ ) are

$$
\begin{gathered}
\left\{\begin{array}{l}
\sigma_{1} \\
\sigma_{2} \\
\sigma_{6}
\end{array}\right\}^{k}=\left[\begin{array}{lll}
Q_{11} & Q_{12} & Q_{16} \\
Q_{12} & Q_{22} & Q_{26} \\
Q_{16} & Q_{26} & Q_{66}
\end{array}\right]^{k}\left\{\begin{array}{l}
\varepsilon_{1} \\
\varepsilon_{2} \\
\gamma_{6}
\end{array}\right\}^{k}-\left[\begin{array}{ccc}
0 & 0 & e_{31} \\
0 & 0 & e_{32} \\
0 & 0 & 0
\end{array}\right]^{k}\left\{\begin{array}{l}
E_{1} \\
\left.E_{2}\right\} \\
E_{3}
\end{array}\right\}^{k} \\
\left\{\begin{array}{l}
\sigma_{4} \\
\sigma_{5}
\end{array}\right\}^{k}=\left[\begin{array}{ll}
Q_{44} & Q_{45} \\
Q_{45} & Q_{55}
\end{array}\right]^{k}\left\{\begin{array}{l}
\gamma_{4} \\
\gamma_{5}
\end{array}\right\}^{k}+\left[\begin{array}{lll}
e_{14} & e_{24} & 0 \\
e_{15} & e_{25} & 0
\end{array}\right]^{k}\left\{\begin{array}{l}
E_{1} \\
E_{2} \\
E_{3}
\end{array}\right\} \\
\left\{\begin{array}{l}
D_{1} \\
D_{2} \\
D_{3}
\end{array}\right\}^{k}=\left[\begin{array}{ccc}
0 & 0 & 0 \\
0 & 0 & 0 \\
e_{31} & e_{32} & 0
\end{array}\right]^{k}\left\{\begin{array}{l}
\varepsilon_{1} \\
\varepsilon_{2} \\
\gamma_{6}
\end{array}\right\}^{k}+\left[\begin{array}{ccc}
\epsilon_{11} & 0 & 0 \\
0 & \epsilon_{22} & 0 \\
0 & 0 & \epsilon_{33}
\end{array}\right]^{k}\left\{\begin{array}{l}
E_{1} \\
E_{2} \\
E_{3}
\end{array}\right\}^{k}
\end{gathered}
$$

For non-piezoelectric layers the terms corresponding to electric field disappear. On transformation to the laminate coordinate system $(x, y, z)$, 


$$
\begin{aligned}
& \left\{\begin{array}{c}
\sigma_{x} \\
\sigma_{y} \\
\tau_{x y}
\end{array}\right\}^{k}=\left[\begin{array}{lll}
\bar{Q}_{11} & \bar{Q}_{12} & \bar{Q}_{16} \\
\bar{Q}_{12} & \bar{Q}_{22} & \bar{Q}_{26} \\
\bar{Q}_{16} & \bar{Q}_{26} & \bar{Q}_{66}
\end{array}\right]^{k}\left\{\begin{array}{c}
\varepsilon_{x} \\
\varepsilon_{y} \\
\gamma_{x y}
\end{array}\right\}^{k}-\left[\begin{array}{lll}
0 & 0 & \overline{\mathrm{e}}_{31} \\
0 & 0 & \overline{\mathrm{e}}_{32} \\
0 & 0 & \overline{\mathrm{e}}_{36}
\end{array}\right]^{k}\left\{\begin{array}{c}
E_{x} \\
E_{y} \\
E_{z}
\end{array}\right\}^{k} \\
& \left\{\begin{array}{l}
\tau_{y z} \\
\tau_{x z}
\end{array}\right\}^{k}=\left[\begin{array}{ll}
\bar{Q}_{44} & \bar{Q}_{45} \\
\bar{Q}_{45} & \bar{Q}_{55}
\end{array}\right]^{k}\left\{\begin{array}{l}
\gamma_{y z} \\
\gamma_{x z}
\end{array}\right\}^{k}+\left[\begin{array}{lll}
\bar{e}_{14} & \bar{e}_{24} & 0 \\
\bar{e}_{15} & \bar{e}_{25} & 0
\end{array}\right]^{k}\left\{\begin{array}{l}
E_{x} \\
E_{y} \\
E_{z}
\end{array}\right\}^{k} \\
& \left\{\begin{array}{l}
D_{x} \\
D_{y} \\
D_{z}
\end{array}\right\}^{k}=\left[\begin{array}{ccc}
0 & 0 & 0 \\
0 & 0 & 0 \\
\bar{e}_{31} & \bar{e}_{32} & \bar{e}_{36}
\end{array}\right]^{k}\left\{\begin{array}{c}
\varepsilon_{x} \\
\varepsilon_{y} \\
\gamma_{x y}
\end{array}\right\}^{k}+\left[\begin{array}{cc}
\bar{e}_{14} & \bar{e}_{15} \\
\bar{e}_{24} & \bar{e}_{25} \\
0 & 0
\end{array}\right]^{k}\left\{\begin{array}{l}
\gamma_{y z} \\
\gamma_{x z}
\end{array}\right\}^{k} \\
& +\left[\begin{array}{ccc}
\bar{\epsilon}_{11} & 0 & 0 \\
0 & \bar{\epsilon}_{22} & 0 \\
0 & 0 & \bar{\epsilon}_{33}
\end{array}\right]^{k}\left\{\begin{array}{l}
E_{x} \\
E_{y} \\
E_{z}
\end{array}\right\}
\end{aligned}
$$

In addition to the stress resultants defined earlier under CLPT, the shear stress resultants $Q_{x}, Q_{y}$ are defined as

$$
\left(Q_{x}, Q_{y}\right)=\int_{-h / 2}^{h / 2}\left(\sigma_{x z}, \sigma_{y z}\right) d z
$$

In the present research $\bar{e}_{14}, \bar{e}_{24}, \bar{e}_{15}$ and $\bar{e}_{25}$ are taken as zero as the piezoelectric layer is formulated to have coupling only in the normal directions. The laminate constitutive equations in terms of force and moment resultant are

$$
\begin{gathered}
\left\{\begin{array}{c}
N_{x} \\
N_{y} \\
N_{x y}
\end{array}\right\}=\left[\begin{array}{lll}
A_{11} & A_{12} & A_{16} \\
A_{12} & A_{22} & A_{26} \\
A_{16} & A_{26} & A_{66}
\end{array}\right]\left\{\begin{array}{c}
\varepsilon_{\mathrm{x}}^{(0)} \\
\varepsilon_{\mathrm{y}}^{(0)} \\
\gamma_{\mathrm{xy}}^{(0)}
\end{array}\right\}+\left[\begin{array}{lll}
B_{11} & B_{12} & B_{16} \\
B_{12} & B_{22} & B_{26} \\
B_{16} & B_{26} & B_{66}
\end{array}\right]\left\{\begin{array}{c}
\varepsilon_{\mathrm{x}}^{(1)} \\
\varepsilon_{\mathrm{y}}^{(1)} \\
\gamma_{\mathrm{xy}}^{(1)}
\end{array}\right\}-\left\{\begin{array}{c}
N_{x}^{P} \\
N_{y}^{P} \\
N_{x y}^{P}
\end{array}\right\} \\
\left\{\begin{array}{c}
M_{x} \\
M_{y} \\
M_{x y}
\end{array}\right\}=\left[\begin{array}{lll}
B_{11} & B_{12} & B_{16} \\
B_{12} & B_{22} & B_{26} \\
B_{16} & B_{26} & B_{66}
\end{array}\right]\left\{\begin{array}{c}
\varepsilon_{\mathrm{x}}^{(0)} \\
\varepsilon_{\mathrm{y}}^{(0)} \\
\gamma_{\mathrm{xy}}^{(0)}
\end{array}\right\}+\left[\begin{array}{lll}
D_{11} & D_{12} & D_{16} \\
D_{12} & D_{22} & D_{26} \\
D_{16} & D_{26} & D_{66}
\end{array}\right]\left\{\begin{array}{c}
\varepsilon_{\mathrm{x}}^{(1)} \\
\varepsilon_{\mathrm{y}}^{(1)} \\
\gamma_{\mathrm{xy}}^{(1)}
\end{array}\right\}-\left\{\begin{array}{c}
M_{x}^{P} \\
M_{y}^{P} \\
M_{x y}^{P}
\end{array}\right\} \\
\left\{\begin{array}{l}
Q_{y} \\
Q_{x}
\end{array}\right\}=K\left[\begin{array}{ll}
A_{44} & A_{45} \\
A_{45} & A_{55}
\end{array}\right]\left\{\begin{array}{c}
\gamma_{y z} \\
\gamma_{x z}
\end{array}\right\}
\end{gathered}
$$


It is seen that the transverse shear strains are constant through the laminate thickness and so are the stresses. But from the elementary theory of homogenous beams, the shear stress variation is parabolic through the beam thickness. To account for this difference, a correction factor $K$ is multiplied to the transverse shear stresses $Q_{y}$ and $Q_{x}$.

The shear stiffness coefficients are obtained as

$$
A_{i j}=\sum_{k=1}^{n} \int_{z_{k-1}}^{z_{k}} Q_{i j}^{k} d z \quad \text { for } i, j=4,5
$$

\subsubsection{Higher order shear deformation theory}

In higher order shear deformation theory (HSDT), the straightness and perpendicularity of the transverse normal after deformation is relaxed as shown in Fig. (3.4) and the displacements are expressed as cubic function of the thickness coordinate. The reason for expanding the displacements up to the cubic term of the thickness coordinate is to have a quadratic variation of the transverse shear stresses through the thickness of the ply, which is more inline with the expected distribution.

The displacement field can be expressed as [Reddy 2004]

$$
\begin{gathered}
u(x, y, z, t)=u_{o}(x, y, t)+z \phi_{x}+z^{2} \theta_{x}+z^{3} \lambda_{x} \\
v(x, y, z, t)=v_{o}(x, y, t)+z \phi_{y}+z^{2} \theta_{y}+z^{3} \lambda_{y} \\
w=w_{o}
\end{gathered}
$$

where $\phi_{x}, \phi_{y}, \theta_{x}, \theta_{y}, \lambda_{x}$ and $\lambda_{y}$ are functions to be determined.

On imposing the condition that the transverse shear stresses $\tau_{y z}$ and $\tau_{x z}$ vanish on the top and bottom surface of the lamina $\left(z= \pm \frac{h}{2}\right)$, the unknown functions in Eq. (3.27) are determined as

$$
\lambda_{x}=-c_{1}\left(\phi_{x}+\frac{\partial w_{o}}{\partial x}\right)
$$




$$
\begin{gathered}
\lambda_{y}=-c_{1}\left(\phi_{y}+\frac{\partial w_{o}}{\partial y}\right) \\
\theta_{x}=0 \\
\theta_{y}=0
\end{gathered}
$$

Therefore the displacement distribution become,

$$
\begin{gathered}
u(x, y, z, t)=u_{o}(x, y, t)+z \phi_{x}(x, y, t)-z^{3} c_{1}\left(\phi_{x}+\frac{\partial w_{o}}{\partial x}\right) \\
v(x, y, z, t)=v_{o}(x, y, t)+z \phi_{y}(x, y, t)-z^{3} c_{1}\left(\phi_{y}+\frac{\partial w_{o}}{\partial y}\right) \\
w=w_{o}=w_{b}+w_{s}
\end{gathered}
$$

where $c_{1}=4 / 3 h^{2}$

$$
\begin{aligned}
& \phi_{x}=-\partial w_{b} / \partial x \\
& \phi_{y}=-\partial w_{b} / \partial y
\end{aligned}
$$

$w_{b}$ is the bending component and $w_{s}$ is the shear component.

Setting $c_{1}=0$ in the above displacement field, it reduces to FSDT 


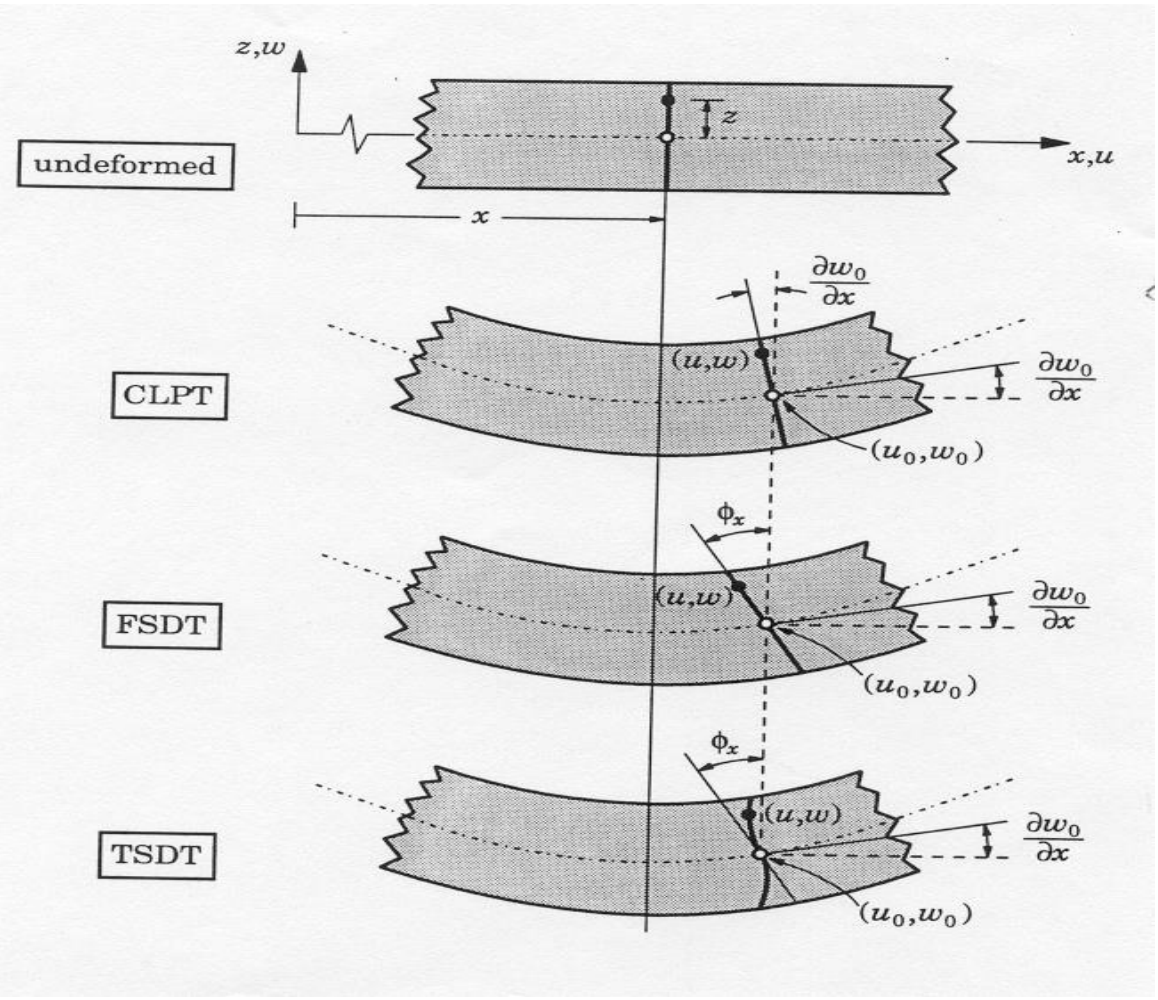

Figure 3.4 Deformation of transverse normal for CLPT, FSDT and HSDT [Reddy (1997)]

The strains associated with the above displacement field are

$$
\begin{gathered}
\left\{\begin{array}{c}
\varepsilon_{\mathrm{x}} \\
\varepsilon_{\mathrm{y}} \\
\gamma_{\mathrm{xy}}
\end{array}\right\}=\left\{\begin{array}{c}
\varepsilon_{\mathrm{x}}^{(0)} \\
\varepsilon_{\mathrm{y}}^{(0)} \\
\gamma_{\mathrm{xy}}^{(0)}
\end{array}\right\}+\mathrm{z}\left\{\begin{array}{c}
\varepsilon_{\mathrm{x}}^{(1)} \\
\varepsilon_{\mathrm{y}}^{(1)} \\
\gamma_{\mathrm{xy}}^{(1)}
\end{array}\right\}+z^{3}\left\{\begin{array}{c}
\varepsilon_{\mathrm{x}}^{(3)} \\
\varepsilon_{\mathrm{y}}^{(3)} \\
\gamma_{\mathrm{xy}}^{(3)}
\end{array}\right\} \\
\left\{\begin{array}{l}
\gamma_{y z} \\
\gamma_{x z}
\end{array}\right\}=\left\{\begin{array}{l}
\gamma_{y z}^{(0)} \\
\gamma_{x z}^{(0)}
\end{array}\right\}+z^{2}\left\{\begin{array}{l}
\gamma_{y z}^{(2)} \\
\gamma_{x z}^{(2)}
\end{array}\right\} \\
\left\{\begin{array}{c}
\varepsilon_{\mathrm{x}}^{(0)} \\
\varepsilon_{\mathrm{y}}^{(0)} \\
\gamma_{\mathrm{xy}}^{(0)}
\end{array}\right\}=\left\{\begin{array}{c}
\frac{\partial u_{o}}{\partial x}+\frac{1}{2}\left(\frac{\partial w_{b}}{\partial x}\right)^{2}+\frac{1}{2}\left(\frac{\partial w_{s}}{\partial x}\right)^{2}+\frac{1}{2}\left(\frac{\partial w_{b}}{\partial y}\right)^{2}+\frac{1}{2}\left(\frac{\partial w_{b}}{\partial y}\right)^{2} \\
\frac{\partial u_{o}}{\partial y}+\frac{\partial v_{o}}{\partial x}+\frac{\partial w_{o}}{\partial x} \frac{\partial w_{o}}{\partial y}
\end{array}\right\}
\end{gathered}
$$




$$
\begin{gathered}
\left\{\begin{array}{c}
\varepsilon_{\mathrm{x}}^{(1)} \\
\varepsilon_{\mathrm{y}}^{(1)} \\
\gamma_{\mathrm{xy}}^{(1)}
\end{array}\right\}=\left\{\begin{array}{c}
-\frac{\partial^{2} w_{b}}{\partial x} \\
-\frac{\partial^{2} w_{b}}{\partial y} \\
-2 \frac{\partial^{2} w_{b}}{\partial x \partial y}
\end{array}\right\} \\
\left\{\begin{array}{c}
\varepsilon_{\mathrm{x}}^{(3)} \\
\varepsilon_{\mathrm{y}}^{(3)} \\
\gamma_{\mathrm{xy}}^{(3)}
\end{array}\right\}=c_{1}\left\{\begin{array}{l}
-\frac{\partial^{2} w_{s}}{\partial x} \\
-\frac{\partial^{2} w_{s}}{\partial y} \\
-2 \frac{\partial^{2} w_{s}}{\partial x \partial y}
\end{array}\right\} \\
\left\{\begin{array}{l}
\gamma_{y z}^{(0)} \\
\gamma_{x z}^{(0)}
\end{array}\right\}=\left\{\begin{array}{l}
\frac{\partial w_{s}}{\partial y} \\
\frac{\partial w_{s}}{\partial x}
\end{array}\right\} \\
\left\{\begin{array}{l}
\gamma_{y z}^{(2)} \\
\gamma_{x z}^{(2)}
\end{array}\right\}=-3 c_{1}\left\{\begin{array}{l}
\frac{\partial w_{s}}{\partial y} \\
\frac{\partial w_{s}}{\partial x}
\end{array}\right\}
\end{gathered}
$$

\section{HSDT Constitutive Equations with Piezoelectric Layers}

The lamina constitutive equations of a $k^{\text {th }}$ piezoelectric lamina coupling the elastic, electric and piezoelectric field in material coordinate system (defined as $x_{1}, x_{2}, x_{3}$ ) are

$$
\begin{gathered}
\left\{\begin{array}{l}
\sigma_{1} \\
\sigma_{2} \\
\sigma_{6}
\end{array}\right\}^{k}=\left[\begin{array}{lll}
Q_{11} & Q_{12} & Q_{16} \\
Q_{12} & Q_{22} & Q_{26} \\
Q_{16} & Q_{26} & Q_{66}
\end{array}\right]^{k}\left\{\begin{array}{l}
\varepsilon_{1} \\
\varepsilon_{2} \\
\gamma_{6}
\end{array}\right\}^{k}-\left[\begin{array}{lll}
0 & 0 & e_{31} \\
0 & 0 & e_{32} \\
0 & 0 & 0
\end{array}\right]^{k}\left\{\begin{array}{l}
E_{1} \\
E_{2} \\
E_{3}
\end{array}\right\}^{k} \\
\left\{\begin{array}{l}
\sigma_{4} \\
\sigma_{5}
\end{array}\right\}^{k}=\left[\begin{array}{ll}
Q_{44} & Q_{45} \\
Q_{45} & Q_{55}
\end{array}\right]^{k}\left\{\begin{array}{l}
\gamma_{4} \\
\gamma_{5}
\end{array}\right\}^{k}-\left[\begin{array}{lll}
e_{14} & e_{24} & 0 \\
e_{15} & e_{25} & 0
\end{array}\right]^{k}\left\{\begin{array}{l}
E_{1} \\
E_{2} \\
E_{3}
\end{array}\right\}^{k}
\end{gathered}
$$




$$
\begin{gathered}
\left\{\begin{array}{l}
D_{1} \\
D_{2} \\
D_{3}
\end{array}\right\}^{k}=\left[\begin{array}{ccc}
0 & 0 & 0 \\
0 & 0 & 0 \\
e_{31} & e_{32} & 0
\end{array}\right]^{k}\left\{\begin{array}{l}
\varepsilon_{1} \\
\varepsilon_{2} \\
\gamma_{6}
\end{array}\right\}^{k}+\left[\begin{array}{cc}
e_{14} & e_{15} \\
e_{24} & e_{25} \\
0 & 0
\end{array}\right]^{k}\left\{\begin{array}{l}
\gamma_{4} \\
\gamma_{5}
\end{array}\right\}^{k} \\
+\left[\begin{array}{ccc}
\epsilon_{11} & 0 & 0 \\
0 & \epsilon_{22} & 0 \\
0 & 0 & \epsilon_{33}
\end{array}\right]^{k}\left\{\begin{array}{l}
E_{1} \\
E_{2} \\
E_{3}
\end{array}\right\}^{k}
\end{gathered}
$$

For non-piezoelectric layers, the terms corresponding to the electric field disappear. The above equations in laminate coordinate system are

$$
\begin{aligned}
& \left\{\begin{array}{c}
\sigma_{x} \\
\sigma_{y} \\
\tau_{x y}
\end{array}\right\}^{k}=\left[\begin{array}{lll}
\bar{Q}_{11} & \bar{Q}_{12} & \bar{Q}_{16} \\
\bar{Q}_{12} & \bar{Q}_{22} & \bar{Q}_{26} \\
\bar{Q}_{16} & \bar{Q}_{26} & \bar{Q}_{66}
\end{array}\right]^{k}\left\{\begin{array}{c}
\varepsilon_{x} \\
\varepsilon_{y} \\
\gamma_{x y}
\end{array}\right\}^{k}-\left[\begin{array}{ccc}
0 & 0 & \overline{\mathrm{e}}_{31} \\
0 & 0 & \overline{\mathrm{e}}_{32} \\
0 & 0 & \overline{\mathrm{e}}_{36}
\end{array}\right]^{k}\left\{\begin{array}{c}
E_{x} \\
E_{y} \\
E_{z}
\end{array}\right\}^{k} \\
& \left\{\begin{array}{l}
\tau_{y z} \\
\tau_{x z}
\end{array}\right\}^{k}=\left[\begin{array}{ll}
\bar{Q}_{44} & \bar{Q}_{45} \\
\bar{Q}_{45} & \bar{Q}_{55}
\end{array}\right]^{k}\left\{\begin{array}{l}
\gamma_{y z} \\
\gamma_{x z}
\end{array}\right\}^{k}+\left[\begin{array}{lll}
\bar{e}_{14} & \bar{e}_{24} & 0 \\
\bar{e}_{15} & \bar{e}_{25} & 0
\end{array}\right]^{k}\left\{\begin{array}{l}
E_{x} \\
E_{y} \\
E_{z}
\end{array}\right\}^{k} \\
& \left\{\begin{array}{l}
D_{x} \\
D_{y} \\
D_{z}
\end{array}\right\}^{k}=\left[\begin{array}{ccc}
0 & 0 & 0 \\
0 & 0 & 0 \\
\bar{e}_{31} & \overline{\mathrm{e}}_{32} & \overline{\mathrm{e}}_{36}
\end{array}\right]^{k}\left\{\begin{array}{c}
\varepsilon_{x} \\
\varepsilon_{y} \\
\gamma_{x y}
\end{array}\right\}^{k}+\left[\begin{array}{cc}
\bar{e}_{14} & \bar{e}_{15} \\
\bar{e}_{24} & \bar{e}_{25} \\
0 & 0
\end{array}\right]^{k}\left\{\begin{array}{l}
\gamma_{y z} \\
\gamma_{x z}
\end{array}\right\}^{k} \\
& +\left[\begin{array}{ccc}
\bar{\epsilon}_{11} & 0 & 0 \\
0 & \bar{\epsilon}_{22} & 0 \\
0 & 0 & \bar{\epsilon}_{33}
\end{array}\right]^{k}\left\{\begin{array}{l}
E_{x} \\
E_{y} \\
E_{z}
\end{array}\right\}^{k}
\end{aligned}
$$

The additional stress resultants are defined as

$$
\begin{aligned}
\left(P_{x}, P_{y}, P_{x y}\right) & =\int_{-h / 2}^{h / 2}\left(\sigma_{x}, \sigma_{y}, \tau_{x y}\right) z^{3} d z \\
\left(R_{x}, R_{y}\right) & =\int_{-h / 2}^{h / 2}\left(\tau_{x z}, \tau_{y z}\right) z^{2} d z
\end{aligned}
$$

where $\left(P_{x}, P_{y}, P_{x y}, R_{x}, R_{y}\right)$ are higher-order stress resultants.

The relation between the stress resultants and the strains are given by 


$$
\begin{aligned}
& \left\{\begin{array}{l}
\{N\} \\
\{M\} \\
\{P\}
\end{array}\right\}=\left[\begin{array}{lll}
{[A]} & {[B]} & {[E]} \\
{[B]} & {[D]} & {[F]} \\
{[E]} & {[F]} & {[H]}
\end{array}\right]\left\{\begin{array}{l}
\left\{\varepsilon^{(0)}\right\} \\
\left\{\varepsilon^{(1)}\right\} \\
\left\{\varepsilon^{(3)}\right\}
\end{array}\right\}-\left\{\begin{array}{l}
\left\{N^{P}\right\} \\
\left\{M^{P}\right\} \\
\left\{P^{P}\right\}
\end{array}\right\} \\
& \left\{\begin{array}{l}
\{Q\} \\
\{R\}
\end{array}\right\}=\left[\begin{array}{ll}
{[A]} & {[D]} \\
{[D]} & {[F]}
\end{array}\right]\left\{\begin{array}{l}
\left\{\gamma^{(0)}\right\} \\
\left\{\gamma^{(2)}\right\}
\end{array}\right\} \\
& \left\{P^{P}\right\}=\sum_{k=1}^{n}(\bar{Q})_{k} t_{k} \bar{Z}_{k}^{3}[d]^{T}{ }_{k}\{E\}_{k}=-\sum_{k=1}^{n}(\bar{Q})_{k} \bar{Z}_{k}^{3}[d]^{T}{ }_{k}\{\varphi\}_{k}
\end{aligned}
$$

The $[E],[F]$ and $[H]$ are higher-order stiffness coefficients. These coefficient matrices are calculated from

$$
\left(E_{i j}, F_{i j}, H_{i j}\right)=\sum_{k=1}^{n} \int_{z_{k-1}}^{z_{k}} \bar{Q}_{i j}^{k}\left(z^{3}, z^{4}, z^{6}\right) d z \text { for } i, j=1,2,6
$$

Shear stiffness coefficients are obtained as

$$
\left(A_{i j}, D_{i j}, F_{i j}\right)=\sum_{k=1}^{n} \int_{z_{k-1}}^{z_{k}} Q_{i j}^{k}\left(1, z^{2}, z^{4}\right) d z \text { for } i, j=4,5
$$

\subsection{Hamilton's principle}

The governing equation of motion is derived from Hamilton's principle, which is stated as

$$
\int_{t_{1}}^{t_{2}}(\delta U-\delta W-\delta T) d t=0
$$

The virtual strain energy $\delta U$ is due to mechanical and electrical strain energy components. Eq. (3.40) can be written as

$$
\int_{t_{1}}^{t_{2}}\left(\left(\delta U_{M}-\delta U_{E}\right)-\delta W-\delta T\right) d t=0
$$

where $\delta U_{M}$ is the virtual mechanical strain energy, $\delta U_{E}$ is the virtual electrical strain energy, $\delta W$ is the virtual work done, and $\delta T$ is the virtual kinetic energy of the system. 


\subsection{Virtual mechanical strain energy for the plate}

The virtual mechanical strain energy of a plate is given by

$$
\delta U_{M}=\iiint_{V}\left(\sigma_{x} \delta \varepsilon_{x}+\sigma_{y} \delta \varepsilon_{y}+\tau_{x y} \delta \gamma_{x y}+\tau_{x z} \delta \gamma_{x z}+\tau_{y z} \delta \gamma_{y z}\right) d V
$$

This can be written in terms of the stress resultants and virtual strains depending on the plate theory in consideration.

\subsubsection{Reduction of Plate Equations to Beam in a Consistent Manner for CLPT [Chandrasekaran (2000)]}

Combining the force and moment resultants in Eq. (3.16), the following equation is obtained.

$$
\left\{\begin{array}{c}
N_{x} \\
N_{y} \\
N_{x y} \\
M_{x} \\
M_{y} \\
M_{x y}
\end{array}\right\}=\left[\begin{array}{llllll}
A_{11} & A_{12} & A_{16} & B_{11} & B_{12} & B_{16} \\
A_{12} & A_{22} & A_{26} & B_{12} & B_{22} & B_{26} \\
A_{16} & A_{26} & A_{66} & B_{16} & B_{26} & B_{66} \\
B_{11} & B_{12} & B_{16} & D_{11} & D_{12} & D_{16} \\
B_{12} & B_{22} & B_{26} & D_{12} & D_{22} & D_{26} \\
B_{16} & B_{26} & B_{66} & D_{16} & D_{26} & D_{66}
\end{array}\right]\left\{\begin{array}{l}
\varepsilon_{x}^{(0)} \\
\varepsilon_{y}^{(0)} \\
\gamma_{x y}^{(0)} \\
\varepsilon_{x}^{(1)} \\
\varepsilon_{y}^{(1)} \\
\gamma_{x y}^{(1)}
\end{array}\right\}-\left\{\begin{array}{c}
N_{x}^{P} \\
N_{y}^{P} \\
N_{x y}^{P} \\
M_{x}^{P} \\
M_{y}^{P} \\
M_{x y}^{P}
\end{array}\right\}
$$

When $N_{y}$ and $M_{y}$ are set to zero. Eq. (3.43) becomes (upon reordering)

$$
\left\{\begin{array}{c}
N_{x} \\
N_{x y} \\
M_{x} \\
M_{x y} \\
0 \\
0
\end{array}\right\}=\left[\begin{array}{llllll}
A_{11} & A_{16} & B_{11} & B_{16} & A_{12} & B_{12} \\
A_{16} & A_{66} & B_{16} & B_{66} & A_{26} & B_{26} \\
B_{11} & B_{16} & D_{11} & D_{16} & B_{12} & D_{12} \\
B_{16} & B_{66} & D_{16} & D_{66} & B_{26} & D_{26} \\
A_{12} & A_{26} & B_{12} & B_{26} & A_{22} & B_{22} \\
B_{12} & B_{26} & D_{12} & D_{26} & B_{22} & D_{22}
\end{array}\right]\left\{\begin{array}{c}
\varepsilon_{x}^{(0)} \\
\gamma_{x y}^{(0)} \\
\varepsilon_{x}^{(1)} \\
\gamma_{x y}^{(1)} \\
\varepsilon_{y}^{(0)} \\
\varepsilon_{y}^{(1)}
\end{array}\right\}-\left\{\begin{array}{c}
N_{x}^{P} \\
N_{x y}^{P} \\
M_{x}^{P} \\
M_{x y}^{P} \\
N_{y}^{P} \\
M_{y}^{P}
\end{array}\right\}
$$


Introducing the notation $\left[\boldsymbol{S}^{\mathbf{1 1}}\right],\left[\boldsymbol{S}^{\mathbf{1 2}}\right],\left[\boldsymbol{S}^{\mathbf{2 1}}\right]$ and $\left[\boldsymbol{S}^{\mathbf{2 2}}\right]$ for the partitions in the $6 \times 6$ matrix of Eq. (3.44),

$$
\begin{aligned}
& \left\{\begin{array}{c}
N_{x} \\
N_{x y} \\
M_{x} \\
M_{x y} \\
0 \\
0
\end{array}\right\}=\left[\begin{array}{ll}
S^{11} & S^{12} \\
S^{21} & S^{22}
\end{array}\right]\left\{\begin{array}{c}
\varepsilon_{x}^{(0)} \\
\gamma_{x y}^{(0)} \\
\varepsilon_{x}^{(1)} \\
\gamma_{x y}^{(1)} \\
\varepsilon_{y}^{(0)} \\
\varepsilon_{y}^{(1)}
\end{array}\right\}-\left\{\begin{array}{c}
N_{x}^{P} \\
N_{x y}^{P} \\
M_{x}^{P} \\
M_{x y}^{P} \\
N_{y}^{P} \\
M_{y}^{P}
\end{array}\right\} \\
& {\left[S^{11}\right]=\left[\begin{array}{llll}
A_{11} & A_{16} & B_{11} & B_{16} \\
A_{16} & A_{66} & B_{16} & B_{66} \\
B_{11} & B_{16} & D_{11} & D_{16} \\
B_{16} & B_{66} & D_{16} & D_{66}
\end{array}\right]} \\
& {\left[S^{12}\right]=\left[\begin{array}{ll}
A_{12} & B_{12} \\
A_{26} & B_{26} \\
B_{12} & D_{12} \\
B_{26} & D_{26}
\end{array}\right]} \\
& {\left[S^{22}\right]=\left[\begin{array}{ll}
A_{22} & B_{22} \\
B_{22} & D_{22}
\end{array}\right]} \\
& {\left[S^{21}\right]=\left[S^{12}\right]^{T}}
\end{aligned}
$$

Introducing the notation $\left\{\{\}_{L}\right\}$ and $\left\{\{\}_{T}\right\}$ to represent the inplane quantities except in the $\mathrm{y}$ dierection and the ones in y direction. The piezoelectric stress resultants are seperated for the actuator and the sensor layers. The actuator and the sensor voltages $\left(\varphi_{a}, \varphi_{s}\right)$ are taken out for simplification purpose.

$$
\left\{\begin{array}{c}
N_{x} \\
N_{x y} \\
M_{x} \\
M_{x y} \\
0 \\
0
\end{array}\right\}=\left[\begin{array}{ll}
S^{11} & S^{12} \\
S^{21} & S^{22}
\end{array}\right]\left\{\begin{array}{l}
\left\{\epsilon_{L}\right\} \\
\left\{\epsilon_{T}\right\}
\end{array}\right\}+\left\{\begin{array}{l}
\left\{N^{P L}\right\} \\
\left\{N^{P T}\right\}
\end{array}\right\}_{a} \varphi_{a}+\left\{\begin{array}{l}
\left\{N^{P L}\right\} \\
\left\{N^{P T}\right\}
\end{array}\right\}_{S} \varphi_{S}
$$




$$
\begin{gathered}
\left\{\epsilon_{L}\right\}=\left\{\begin{array}{c}
\varepsilon_{x}^{(0)} \\
\gamma_{x y}^{(0)} \\
\varepsilon_{x}^{(1)} \\
\gamma_{x y}^{(1)}
\end{array}\right\},\left\{\epsilon_{T}\right\}=\left\{\begin{array}{c}
\varepsilon_{y}^{(0)} \\
\varepsilon_{y}^{(1)}
\end{array}\right\}, \\
\left\{N^{P L}\right\}_{a}=\frac{-1}{\varphi_{a}}\left\{\begin{array}{c}
N_{x}^{P} \\
N_{x y}^{P} \\
M_{x}^{P} \\
M_{x y}^{P}
\end{array}\right\}_{a},\left\{N^{P T}\right\}_{a}=\frac{-1}{\varphi_{a}}\left\{\begin{array}{l}
N_{y}^{P} \\
M_{y}^{P}
\end{array}\right\}_{a} \\
\left\{N^{P L}\right\}_{s}=\frac{-1}{\varphi_{s}}\left\{\begin{array}{c}
N_{x}^{P} \\
N_{x y}^{P} \\
M_{x}^{P} \\
M_{x y}^{P}
\end{array}\right\}_{s},\left\{N^{P T}\right\}_{s}=\frac{-1}{\varphi_{s}}\left\{\begin{array}{l}
N_{y}^{P} \\
M_{y}^{P}
\end{array}\right\}_{s}
\end{gathered}
$$

The subscripts ' $s$ ' and ' $a$ ' denote sensor and actuator respectively.

Eliminating the last two rows of Eq. (3.44),

$$
\left\{\begin{array}{c}
N_{x} \\
N_{x y} \\
M_{x} \\
M_{x y}
\end{array}\right\}=[\bar{S}]\left\{\epsilon_{L}\right\}+\left\{\bar{S}_{P}\right\}_{a} \varphi_{a}+\left\{\bar{S}_{P}\right\}_{s} \varphi_{s}
$$

where $[\bar{S}]=\left[S^{11}\right]-\left[S^{12}\right]\left[S^{22}\right]^{-1}\left[S^{21}\right]$

$$
\begin{aligned}
& \left\{\bar{S}_{P}\right\}_{a}=\left\{N^{P L}\right\}_{a}-\left[S^{12}\right]\left[S^{22}\right]^{-1}\left\{N^{P T}\right\}_{a} \\
& \left\{\bar{S}_{P}\right\}_{S}=\left\{N^{P L}\right\}_{S}-\left[S^{12}\right]\left[S^{22}\right]^{-1}\left\{N^{P T}\right\}_{S}
\end{aligned}
$$

The virtual mechanical strain energy expression of Eq. (3.42) for a beam becomes

$$
\delta U_{M}=b \int_{0}^{L}\left(N_{x} \delta \varepsilon_{x}^{(0)}+N_{x y} \delta \gamma_{x y}^{(0)}+M_{x} \delta \varepsilon_{x}^{(1)}+M_{x y} \delta \gamma_{x y}^{(1)}\right) d x
$$

where $b$ is the width of the beam and $L$ is the length of the beam.

The virtual quantities of the strains in Eq. (3.8) can be written as 


$$
\begin{aligned}
& \left\{\begin{array}{l}
\delta \varepsilon_{x x}^{(0)} \\
\delta \gamma_{x y}^{(0)}
\end{array}\right\}=\left\{\begin{array}{c}
\delta u_{o}^{\prime}+w^{\prime} \delta w^{\prime} \\
\delta \gamma_{o}
\end{array}\right\} \\
& \left\{\begin{array}{c}
\delta \varepsilon_{\mathrm{xx}}^{(1)} \\
\delta \gamma_{\mathrm{xy}}^{(1)}
\end{array}\right\}=\left\{\begin{array}{c}
-\delta w^{\prime \prime} \\
-2 \delta w^{\prime} y
\end{array}\right\}
\end{aligned}
$$

The ()$^{\prime},()^{y}$ represent the partial derivative with respect to $x$ and $y$, respectively and $\gamma_{o}$ is the inplane shear strain. The virtual strain energy is expressed as a function of displacements

$$
\begin{gathered}
\delta U_{M}=b \int_{0}^{L}\left(N_{x}\left(\delta u_{o}^{\prime}+w^{\prime} \delta w^{\prime}\right)+N_{x y} \delta \gamma_{o}+M_{x}\left(-\delta w^{\prime \prime}\right)\right. \\
\left.+M_{x y}\left(-2 \delta w^{\prime y}\right)\right) d x
\end{gathered}
$$

\subsubsection{Reduction of Plate Equations to Beam in a Consistent Manner for FSDT}

Combining the inplane stress results in Eq. (3.25), the following equation is obtained.

$$
\left\{\begin{array}{c}
N_{x} \\
N_{y} \\
N_{x y} \\
M_{x} \\
M_{y} \\
M_{x y}
\end{array}\right\}=\left[\begin{array}{llllll}
A_{11} & A_{12} & A_{16} & B_{11} & B_{12} & B_{16} \\
A_{12} & A_{22} & A_{26} & B_{12} & B_{22} & B_{26} \\
A_{16} & A_{26} & A_{66} & B_{16} & B_{26} & B_{66} \\
B_{11} & B_{12} & B_{16} & D_{11} & D_{12} & D_{16} \\
B_{12} & B_{22} & B_{26} & D_{12} & D_{22} & D_{26} \\
B_{16} & B_{26} & B_{66} & D_{16} & D_{26} & D_{66}
\end{array}\right]\left\{\begin{array}{c}
\varepsilon_{x}^{(0)} \\
\varepsilon_{y}^{(0)} \\
\gamma_{x y}^{(0)} \\
\varepsilon_{x}^{(1)} \\
\varepsilon_{y}^{(1)} \\
\gamma_{x y}^{(1)}
\end{array}\right\}-\left\{\begin{array}{c}
N_{x}^{P} \\
N_{y}^{P} \\
N_{x y}^{P} \\
M_{x}^{P} \\
M_{y}^{P} \\
M_{x y}^{P}
\end{array}\right\}
$$

Setting $N_{y}$ and $M_{y}$ to zero and rearranging the elements of Eq. (3.53)

$$
\left\{\begin{array}{c}
N_{x} \\
N_{x y} \\
M_{x} \\
M_{x y} \\
0 \\
0
\end{array}\right\}=\left[\begin{array}{llllll}
A_{11} & A_{16} & B_{11} & B_{16} & A_{12} & B_{12} \\
A_{16} & A_{66} & B_{16} & B_{66} & A_{26} & B_{26} \\
B_{11} & B_{16} & D_{11} & D_{16} & B_{12} & D_{12} \\
B_{16} & B_{66} & D_{16} & D_{66} & B_{26} & D_{26} \\
A_{12} & A_{26} & B_{12} & B_{26} & A_{22} & B_{22} \\
B_{12} & B_{26} & D_{12} & D_{26} & B_{22} & D_{22}
\end{array}\right]\left\{\begin{array}{c}
\varepsilon_{x}^{(0)} \\
\gamma_{x y}^{(0)} \\
\varepsilon_{x}^{(1)} \\
\gamma_{x y}^{(1)} \\
\varepsilon_{y}^{(0)} \\
\varepsilon_{y}^{(1)}
\end{array}\right\}-\left\{\begin{array}{c}
N_{x}^{P} \\
N_{x y}^{P} \\
M_{x}^{P} \\
M_{x y}^{P} \\
N_{y}^{P} \\
M_{y}^{P}
\end{array}\right\}
$$




$$
\left\{\begin{array}{c}
N_{x} \\
N_{x y} \\
M_{x} \\
M_{x y} \\
0 \\
0
\end{array}\right\}=\left[\begin{array}{ll}
S^{11} & S^{12} \\
S^{21} & S^{22}
\end{array}\right]\left\{\begin{array}{c}
\varepsilon_{x}^{(0)} \\
\gamma_{x y}^{(0)} \\
\varepsilon_{x}^{(1)} \\
\gamma_{x y}^{(1)} \\
\varepsilon_{y}^{(0)} \\
\varepsilon_{y}^{(1)}
\end{array}\right\}-\left\{\begin{array}{c}
N_{x}^{P} \\
N_{x y}^{P} \\
M_{x}^{P} \\
M_{x y}^{P} \\
N_{y}^{P} \\
M_{y}^{P}
\end{array}\right\}
$$

Reducing the plate formulation to beam for FSDT for inplane stresses is same as CLPT and the final equation is given as,

$$
\left\{\begin{array}{c}
N_{x} \\
N_{x y} \\
M_{x} \\
M_{x y}
\end{array}\right\}=[\bar{S}]\left\{\epsilon_{L}\right\}+\left\{\bar{S}_{P}\right\}_{a} \varphi_{a}+\left\{\bar{S}_{P}\right\}_{s} \varphi_{s}
$$

where $[\bar{S}]=\left[S^{11}\right]-\left[S^{12}\right]\left[S^{22}\right]^{-1}\left[S^{21}\right]$

$$
\begin{aligned}
& \left\{\bar{S}_{P}\right\}_{a}=\left\{N^{P L}\right\}_{a}-\left[S^{12}\right]\left[S^{22}\right]^{-1}\left\{N^{P T}\right\}_{a} \\
& \left\{\bar{S}_{P}\right\}_{s}=\left\{N^{P L}\right\}_{S}-\left[S^{12}\right]\left[S^{22}\right]^{-1}\left\{N^{P T}\right\}_{S}
\end{aligned}
$$

The transverse shearstrain in Eq. (3.25) can be written as

$$
\left\{\begin{array}{c}
0 \\
Q_{x}
\end{array}\right\}=K\left[\begin{array}{ll}
A_{44} & A_{45} \\
A_{45} & A_{55}
\end{array}\right]\left\{\begin{array}{c}
\gamma_{y z} \\
\gamma_{x z}
\end{array}\right\}
$$

Then Eq. (3.57) reduces to

$$
Q_{x}=K A^{*} \gamma_{x z}
$$

Where $A^{*}=\left(A_{55}-A_{45}{ }^{2} / A_{44}\right)$ and $K$ is the shear correction factor.

The correction factor $K$ is computed such that the strain energy due to the transverse shears is equal to the strain energy due to true transverse shears predicted by the three dimensional elasticity theory. For a rectangular beam it is taken as $5 / 6$.

The virtual strain energy expression for a beam becomes 


$$
\begin{gathered}
\delta U_{M}=b \int_{0}^{L}\left(N_{x} \delta \varepsilon_{x}^{(0)}+N_{x y} \delta \gamma_{x y}^{(0)}+M_{x} \delta \varepsilon_{x}^{(1)}+M_{x y} \delta \gamma_{x y}^{(1)}\right. \\
\left.+Q_{x} \delta \gamma_{x z}\right) d x
\end{gathered}
$$

The virtual quantities of the strains in Eq. (3.20) can be written as

$$
\begin{gathered}
\left\{\begin{array}{c}
\delta \varepsilon_{x x}^{(0)} \\
\delta \gamma_{x y}^{(0)}
\end{array}\right\}=\left\{\begin{array}{c}
\delta u_{o}^{\prime}+w_{b}^{\prime} \delta w_{b}^{\prime}+w_{s}^{\prime} \delta w_{s}^{\prime} \\
\delta \gamma_{o}
\end{array}\right\} \\
\left\{\begin{array}{l}
\delta \varepsilon_{\mathrm{xx}}^{(1)} \\
\delta \gamma_{\mathrm{xy}}^{(1)}
\end{array}\right\}=\left\{\begin{array}{c}
-\delta w_{b}^{\prime \prime} \\
-2 \delta w_{b}^{\prime y}
\end{array}\right\} \\
\left\{\delta \gamma_{x z}^{(0)}\right\}=\left\{\delta w_{s}^{\prime}\right\}
\end{gathered}
$$

Substituting the above expression in the strain energy expression [Eq. (3.59)], we get

$$
\begin{gathered}
\delta U_{M}=b \int_{0}^{L}\left(N_{x}\left(\delta u_{o}^{\prime}+w_{b}^{\prime} \delta w_{b}^{\prime}+w_{s}^{\prime} \delta w_{s}^{\prime}\right)+N_{x y} \delta \gamma_{o}+M_{x}\left(-\delta w_{b}^{\prime \prime}\right)\right. \\
\left.+M_{x y}\left(-2 \delta w_{b}^{\prime y}\right)+Q_{x} \delta w_{s}^{\prime}\right) d x
\end{gathered}
$$

\subsubsection{Reduction of Plate Equations to Beam in a Consistent Manner for HSDT (Nagappan, 2004)}

Setting $N_{y}, M_{y}$ and $P_{y}$ and rearranging the elements of inplane stress resultants of Eq. (3.36)

$$
\left\{\begin{array}{c}
N_{x} \\
N_{x y} \\
M_{x} \\
M_{x y} \\
P_{x} \\
P_{x y} \\
0 \\
0 \\
0
\end{array}\right\}=\left[\begin{array}{lllllllll}
A_{11} & A_{16} & B_{11} & B_{16} & E_{11} & E_{16} & A_{12} & B_{12} & E_{12} \\
A_{16} & A_{66} & B_{16} & B_{66} & E_{16} & E_{66} & A_{26} & B_{26} & E_{26} \\
B_{11} & B_{16} & D_{11} & D_{16} & F_{11} & F_{16} & B_{12} & D_{12} & F_{12} \\
B_{16} & B_{66} & D_{16} & D_{66} & F_{16} & F_{66} & B_{26} & D_{26} & F_{26} \\
E_{11} & E_{16} & F_{11} & F_{16} & H_{11} & H_{16} & E_{12} & F_{12} & H_{12} \\
E_{16} & E_{66} & F_{16} & F_{66} & H_{16} & H_{66} & E_{26} & F_{26} & H_{26} \\
A_{12} & A_{26} & B_{12} & B_{26} & E_{12} & E_{26} & A_{22} & B_{22} & E_{22} \\
B_{12} & B_{26} & D_{12} & D_{26} & F_{12} & F_{26} & B_{22} & D_{22} & F_{22} \\
E_{12} & E_{26} & F_{12} & F_{26} & H_{12} & H_{26} & E_{22} & F_{22} & H_{22}
\end{array}\right]\left\{\begin{array}{c}
\varepsilon_{x}^{(0)} \\
\gamma_{x y}^{(0)} \\
\varepsilon_{x}^{(1)} \\
\gamma_{x y}^{(1)} \\
\varepsilon_{x}^{(3)} \\
\gamma_{x y}^{(3)} \\
\varepsilon_{y}^{(0)} \\
\varepsilon_{y}^{(1)} \\
\varepsilon_{y}^{(3)}
\end{array}\right\}-\left\{\begin{array}{c}
N_{x}^{P} \\
N_{x y}^{P} \\
M_{x}^{P} \\
M_{x y}^{P} \\
P_{x}^{P} \\
P_{x y}^{P} \\
N_{y}^{P} \\
M_{y}^{P} \\
P_{y}^{P}
\end{array}\right\}
$$

Eliminating the last 3 rows, 


$$
\left\{\begin{array}{c}
N_{x} \\
N_{x y} \\
M_{x} \\
M_{x y} \\
P_{x} \\
P_{x y}
\end{array}\right\}=\left[\begin{array}{ll}
{\left[T^{11}\right]} & {\left[T^{12}\right]} \\
{\left[T^{21}\right]} & {\left[T^{22}\right]}
\end{array}\right]\left\{\begin{array}{l}
\left\{\epsilon_{L}\right\} \\
\left\{\epsilon_{T}\right\}
\end{array}\right\}+\left\{\begin{array}{l}
\left\{N^{P L}\right\} \\
\left\{N^{P T}\right\}
\end{array}\right\}_{a} \varphi_{a}+\left\{\begin{array}{l}
\left\{N^{P L}\right\} \\
\left\{N^{P T}\right\}
\end{array}\right\}_{S} \varphi_{s}
$$

where

$$
\begin{gathered}
{\left[T^{11}\right]=\left[\begin{array}{llllll}
A_{11} & A_{16} & B_{11} & B_{16} & E_{11} & E_{16} \\
A_{16} & A_{66} & B_{16} & B_{66} & E_{16} & E_{66} \\
B_{11} & B_{16} & D_{11} & D_{16} & F_{11} & F_{16} \\
B_{16} & B_{66} & D_{16} & D_{66} & F_{16} & F_{66} \\
E_{11} & E_{16} & F_{11} & F_{16} & H_{11} & H_{16} \\
E_{16} & E_{66} & F_{16} & F_{66} & H_{16} & H_{66}
\end{array}\right]} \\
{\left[T^{12}\right]=\left[\begin{array}{llll}
A_{12} & B_{12} & E_{12} \\
A_{26} & B_{26} & E_{26} \\
B_{12} & D_{12} & F_{12} \\
B_{26} & D_{26} & F_{26} \\
E_{12} & F_{12} & H_{12} \\
E_{26} & F_{26} & H_{26}
\end{array}\right]} \\
{\left[T^{21}\right]=\left[\begin{array}{lll}
T^{12} \\
]^{T}
\end{array}\right.} \\
{\left[T^{22}\right]=\left[\begin{array}{llll}
A_{22} & B_{22} & E_{22} \\
B_{22} & D_{22} & F_{22} \\
E_{22} & F_{22} & H_{22}
\end{array}\right]}
\end{gathered}
$$

$$
\left\{\epsilon^{L}\right\}=\left\{\begin{array}{c}
\varepsilon_{x}^{(0)} \\
\gamma_{x y}^{(0)} \\
\varepsilon_{x}^{(1)} \\
\gamma_{x y}^{(1)} \\
\varepsilon_{x}^{(3)} \\
\gamma_{x y}^{(3)}
\end{array}\right\} \text { and }\left\{\epsilon^{T}\right\}=\left\{\begin{array}{c}
\varepsilon_{y}^{(0)} \\
\varepsilon_{y}^{(1)} \\
\varepsilon_{y}^{(3)}
\end{array}\right\}
$$




$$
\begin{gathered}
\left\{N^{P L}\right\}_{a}=\frac{-1}{\varphi_{a}}\left\{\begin{array}{c}
N_{x}^{P} \\
N_{x y}^{P} \\
M_{x}^{P} \\
M_{x y}^{P} \\
P_{x}^{P} \\
P_{x y}^{P}
\end{array}\right\}_{a} \text { and }\left\{N^{P T}\right\}_{a}=\frac{-1}{\varphi_{a}}\left\{\begin{array}{c}
N_{y}^{P} \\
M_{y}^{P} \\
P_{y}^{P}
\end{array}\right\}_{a} \\
\left\{N^{P L}\right\}_{s}=\frac{-1}{\varphi_{s}}\left\{\begin{array}{c}
N_{x}^{P} \\
N_{x y}^{P} \\
M_{x}^{P} \\
M_{x y}^{P} \\
P_{x}^{P} \\
P_{x y}^{P}
\end{array}\right\}_{s}
\end{gathered}
$$

The reduced beam equations becomes

$$
\left\{\begin{array}{c}
N_{x} \\
N_{x y} \\
M_{x} \\
M_{x y} \\
P_{x} \\
P_{x y}
\end{array}\right\}=[\bar{T}]\left\{\epsilon^{L}\right\}+\left\{\bar{T}_{P}\right\}_{a} \varphi_{a}+\left\{\bar{T}_{P}\right\}_{s} \varphi_{s}
$$

Where $[\bar{T}]=\left[T^{11}\right]-\left[T^{12}\right]\left[T^{22}\right]^{-1}\left[T^{21}\right]$

$$
\begin{aligned}
& \left\{\bar{T}_{P}\right\}_{\boldsymbol{a}}=\left\{N^{P L}\right\}_{\boldsymbol{a}}-\left[T^{12}\right]\left[T^{22}\right]^{-1}\left\{N^{P T}\right\}_{\boldsymbol{a}} \\
& \left\{\bar{T}_{P}\right\}_{s}=\left\{N^{P L}\right\}_{\boldsymbol{s}}-\left[T^{12}\right]\left[T^{22}\right]^{-1}\left\{N^{P T}\right\}_{\boldsymbol{s}}
\end{aligned}
$$

Similarly, rearranging the elements of transverse shear resultants of Eq. (3.36) and setting $Q_{y}$ and $R_{y}$ to zero, we get

$$
\left\{\begin{array}{l}
Q_{x} \\
R_{x} \\
Q_{y} \\
R_{y}
\end{array}\right\}=\left[\begin{array}{llll}
A_{55} & D_{55} & A_{45} & D_{45} \\
D_{55} & F_{55} & D_{45} & F_{45} \\
A_{45} & D_{45} & A_{44} & D_{44} \\
D_{45} & F_{45} & D_{44} & F_{44}
\end{array}\right]\left\{\begin{array}{c}
\gamma_{x z}^{(0)} \\
\gamma_{x z}^{(2)} \\
\gamma_{y z}^{(0)} \\
\gamma_{y z}^{(2)}
\end{array}\right\}
$$

Substituting the kinematic relations in the above equation, 


$$
\left\{\begin{array}{l}
Q_{x} \\
R_{x} \\
Q_{y} \\
R_{y}
\end{array}\right\}=\left[\begin{array}{llll}
A_{55} & D_{55} & A_{45} & D_{45} \\
D_{55} & F_{55} & D_{45} & F_{45} \\
A_{45} & D_{45} & A_{44} & D_{44} \\
D_{45} & F_{45} & D_{44} & F_{44}
\end{array}\right]\left\{\begin{array}{c}
w_{s}^{\prime} \\
-3 c_{1} w_{s}^{\prime} \\
w_{s}^{y} \\
-3 c_{1} w_{s}^{y}
\end{array}\right\}
$$

Let

$D_{i j}^{*}=A_{i j}-6 c_{1} D_{i j}+9 c_{1}^{2} F_{i j}$

$Q_{x}^{*}=Q_{x}-3 c_{1} R_{x}$

$Q_{y}^{*}=Q_{y}-3 c_{1} R_{y}$

Eq. (3.67) can be rewritten as

$$
\left\{\begin{array}{l}
Q_{y}^{*} \\
Q_{x}^{*}
\end{array}\right\}=\left[\begin{array}{ll}
D_{44}^{*} & D_{45}^{*} \\
D_{45}^{*} & D_{55}^{*}
\end{array}\right]\left\{\begin{array}{c}
w_{s}^{y} \\
w_{s}^{\prime}
\end{array}\right\}
$$

Setting $Q_{y}^{*}=0$, Eq. (3.68) reduces to

$Q_{x}^{*}=D_{55}^{* *} w_{s}^{\prime}$

Where $D_{55}^{* *}=D_{55}^{*}-\frac{D_{45}^{* 2}}{D_{44}^{*}}$

The virtual mechanical strain energy expression becomes

$$
\begin{aligned}
\delta U_{M}=b \int_{0}^{L}( & N_{x} \delta \varepsilon_{x}^{(0)}+N_{x y} \delta \gamma_{x y}^{(0)}+M_{x} \delta \varepsilon_{x}^{(1)}+M_{x y} \delta \gamma_{x y}^{(1)}+P_{x} \delta \varepsilon_{x}^{(3)}+P_{x y} \delta \gamma_{x y}^{(3)} \\
& \left.+Q_{x}^{*} \delta \gamma_{x z}^{(0)}\right) d x
\end{aligned}
$$

The virtual quantities of the strains in Eq. (3.31) are 


$$
\begin{gathered}
\left\{\begin{array}{c}
\delta \varepsilon_{x x}^{(0)} \\
\delta \gamma_{x y}^{(0)}
\end{array}\right\}=\left\{\begin{array}{c}
\left.\delta u_{0}^{\prime}+w_{b}^{\prime} \delta w_{b}^{\prime}+w_{s}^{\prime} \delta w_{s}^{\prime}\right\} \\
\delta \gamma_{0}
\end{array}\right\} \\
\left\{\begin{array}{l}
\delta \varepsilon_{\mathrm{xx}}^{(1)} \\
\delta \gamma_{\mathrm{xy}}^{(1)}
\end{array}\right\}=\left\{\begin{array}{c}
-\delta w_{b}^{\prime \prime} \\
-2 \delta w_{b}^{\prime y}
\end{array}\right\} \\
\left\{\begin{array}{l}
\delta \varepsilon_{\mathrm{xx}}^{(3)} \\
\delta \gamma_{\mathrm{xy}}^{(3)}
\end{array}\right\}=c_{1}\left\{\begin{array}{l}
-\delta w_{s}^{\prime \prime} \\
-2 \delta w_{s}^{\prime y}
\end{array}\right\} \\
\left\{\delta \gamma_{x z}^{(0)}\right\}=\left\{\delta w_{s}^{\prime}\right\} \\
\left\{\delta \gamma_{x z}^{(2)}\right\}=-3 c_{1}\left\{\delta w_{s}^{\prime}\right\}
\end{gathered}
$$

Substituting the strain displacement relation in Eq. (3.69), we get the virtual strain energyexpression as

$$
\begin{aligned}
\delta U_{M}=b \int_{0}^{L}[ & N_{x}\left(\delta u_{0}^{\prime}+w_{b}^{\prime} \delta w_{b}^{\prime}+w_{s}^{\prime} \delta w_{s}^{\prime}\right)+N_{x y} \delta \gamma_{0}+M_{x}\left(-\delta w_{b}^{\prime \prime}\right) \\
& +M_{x y}\left(-2 \delta w_{b}^{\prime y}\right)+P_{x}\left(-c_{1} \delta w_{s}^{\prime \prime}\right)+P_{x y}\left(-2 c_{1} \delta w_{s}^{\prime y}\right) \\
& \left.+Q_{x}^{*} \delta w_{s}^{\prime}\right] d x
\end{aligned}
$$

\subsection{Virtual electrical strain energy of a composite plate}

The virtual electrical strain energy is given by

$$
\delta U_{E}=\iiint_{V} D \delta E d V
$$

As the electric field is applied only in the thickness direction, dielectric displacement is seen only in the $z$ direction. Therefore the above equation reduces to

$$
\delta U_{E}=\iiint_{V} D_{z} \delta E_{z} d V
$$

Splitting the volume integral into integral over thickness, length and width 


$$
\delta U_{E}=b \int_{0}^{L} \int_{-h / 2}^{h / 2} D_{z} \delta E_{z} d z d x
$$

Dielectric displacement in $z$ direction can be obtained from Eq. (3.12),

$$
D_{z}=\bar{e}_{31} \varepsilon_{x}+\bar{e}_{32} \varepsilon_{y}+\bar{e}_{36} \gamma_{x y}+\bar{\epsilon}_{33} E_{z}
$$

Substitute Eq. (3.75) in Eq. (3.74), and expressing electric field in terms of potential difference,

$$
\delta U_{E}=-b \int_{0}^{L} \int_{-h / 2}^{h / 2}\left(\bar{e}_{31} \varepsilon_{x}+\bar{e}_{32} \varepsilon_{y}+\bar{e}_{36} \gamma_{x y}-\bar{\epsilon}_{33} \frac{\varphi}{t_{p}}\right) \frac{\delta \varphi}{t_{p}} d Z
$$

\subsubsection{Virtual electrical strain energy of a composite beam using CLPT}

Expanding the strains in Eq. (3.76) in terms of membrane and bending strains

$$
\begin{gathered}
\delta U_{E}=-b \int_{0}^{L} \int_{-h / 2}^{h / 2}\left(\bar{e}_{31}\left(\varepsilon_{x}^{(0)}+z \varepsilon_{x}^{(1)}\right)+\bar{e}_{32}\left(\varepsilon_{y}^{(0)}+z \varepsilon_{y}^{(1)}\right)\right. \\
\left.+\bar{e}_{36}\left(\gamma_{x y}{ }^{(0)}+z \gamma_{x y}{ }^{(1)}\right)-\bar{\epsilon}_{33} \frac{\varphi}{t_{p}}\right) \frac{\delta \varphi}{t_{p}} d Z
\end{gathered}
$$

Further reducing Eq. (3.77) to one dimensional problem using partial plane stress approximation,

$$
\left.\delta U_{E}=-\frac{b}{t_{p}} \int_{0}^{L}\left(\mid D_{s \varphi}\right]\left\{\varepsilon^{L}\right\}-D_{\varphi} \varphi-\bar{\epsilon}_{33} \varphi\right) \delta \varphi
$$

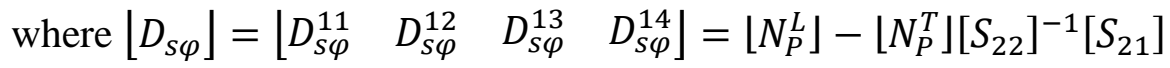

$$
D_{\varphi}=\left\lfloor N_{P}^{T}\right\rfloor\left[S_{22}\right]^{-1}\left\{N_{P}^{T}\right\}
$$

$\left\lfloor N_{P}^{T}\right\rfloor,\left\lfloor N_{P}^{L}\right\rfloor,\left[S_{21}\right]$ and $\left[S_{22}\right]^{-1}$ are from Eq. (3.46) and Eq. (3.48)

Expanding the terms 


$$
\begin{gathered}
\delta U_{E}=-\frac{b}{t_{p}} \int_{0}^{L}\left(D_{s \varphi}^{11} \varepsilon_{x}^{(0)}+D_{s \varphi}^{12} \gamma_{x y}^{(0)}+D_{s \varphi}^{13} \varepsilon_{x}^{(1)}+D_{s \varphi}^{14} \gamma_{x y}^{(1)}-D_{\varphi} \varphi\right. \\
\left.-\bar{\epsilon}_{33} \varphi\right) \delta \varphi
\end{gathered}
$$

Substituting the strain displacement relations into Eq. (3.79)

$$
\begin{gathered}
\delta U_{E}=-\frac{b}{t_{p}} \int_{0}^{L}\left(D_{s \varphi}^{11} u_{0}^{\prime}+D_{s \varphi}^{12} \gamma-D_{s \varphi}^{13} w^{\prime \prime}-2 D_{s \varphi}^{14} w_{y}^{\prime}-D_{\varphi} \varphi\right. \\
\left.-\bar{\epsilon}_{33} \varphi\right) \delta \varphi
\end{gathered}
$$

\subsubsection{Virtual electrical strain energy of a composite beam using FSDT:}

The virtual electrical strain energy is the same as for CLPT since there is no coupling between the transverse shear and piezoelectric terms.

\subsubsection{Virtual electrical strain energy of a composite beam using HSDT:}

Expanding the strains in Eq. (3.76)

$$
\begin{aligned}
\delta U_{E}=-b \int_{0}^{L} \int_{-h / 2}^{h / 2}\left(\bar{e}_{31}\left(\varepsilon_{x}^{(0)}+z \varepsilon_{x}^{(1)}+z^{3} \varepsilon_{x}^{(3)}\right)\right. \\
+\bar{e}_{32}\left(\varepsilon_{y}^{(0)}+z \varepsilon_{y}^{(1)}+z^{3} \varepsilon_{y}^{(3)}\right) \\
\left.+\bar{e}_{36}\left(\gamma_{x y}{ }^{(0)}+z \gamma_{x y}{ }^{(1)}+z^{3} \gamma_{x y}^{(3)}\right)-\bar{\epsilon}_{33} \frac{\varphi}{t_{p}}\right) \frac{\delta \varphi}{t_{p}} d Z
\end{aligned}
$$

Further reducing Eq. (3.81) to one dimensional problem using partial plane stress approximation,

$$
\delta U_{E}=-\frac{b}{t_{p}} \int_{0}^{L}\left(\left|D_{s \varphi}\right|\left\{\varepsilon^{L}\right\}-D_{\varphi} \varphi-\bar{\epsilon}_{33} \varphi\right) \delta \varphi
$$

Where $\left\lfloor D_{s \varphi}\right\rfloor=\left\lfloor\begin{array}{llllll}D_{S \varphi}^{11} & D_{S \varphi}^{12} & D_{S \varphi}^{13} & D_{S \varphi}^{14} & D_{S \varphi}^{15} & D_{S \varphi}^{16}\end{array}\right\rfloor=\left\lfloor N_{P}^{L}\right\rfloor-\left\lfloor N_{P}^{T}\right\rfloor\left[T_{22}\right]^{-1}\left[T_{21}\right]$

$$
D_{\varphi}=\left\lfloor N_{P}^{T}\right]\left[T_{22}\right]^{-1}\left\{N_{P}^{T}\right\}
$$


$\left\lfloor N_{P}^{T}\right\rfloor,\left\lfloor N_{P}^{L}\right\rfloor,\left[T_{21}\right]$ and $\left[T_{22}\right]^{-1}$ are from Eq. (3.64)

Expanding the terms

$$
\begin{gathered}
\delta U_{E}=-\frac{b}{t_{p}} \int_{0}^{L}\left(D_{s \varphi}^{11} \varepsilon_{x}^{(0)}+D_{s \varphi}^{12} \gamma_{x y}^{(0)}+D_{s \varphi}^{13} \varepsilon_{x}^{(1)}+D_{s \varphi}^{14} \gamma_{x y}^{(1)}+D_{s \varphi}^{15} \varepsilon_{x}^{(3)}+D_{s \varphi}^{16} \gamma_{x y}^{(3)}\right. \\
\left.\quad-D_{\varphi} \varphi-\bar{\epsilon}_{33} \varphi\right) \delta \varphi
\end{gathered}
$$

Substituting the strain displacement relations into Eq. (3.83)

$$
\begin{aligned}
\delta U_{E}=-\frac{b}{t_{p}} \int_{0}^{L} & \left(D_{s \varphi}^{11} u_{0}^{\prime}+D_{s \varphi}^{12} \gamma-D_{s \varphi}^{13} w_{b}^{\prime \prime}-2 D_{s \varphi}^{14} w_{b}^{\prime y}\right. \\
& \left.\quad-c_{1} D_{s \varphi}^{15} w_{s}^{\prime \prime}-2 c_{1} D_{s \varphi}^{16} w_{s}^{\prime y}-D_{\varphi} \varphi-\bar{\epsilon}_{33} \varphi\right) \delta \varphi
\end{aligned}
$$

\subsection{Virtual Kinetic energy of a composite beam [Nagappan (2004)]}

The virtual kinetic energy of a plate is given by

$$
\delta T=\iiint_{V} \rho[\dot{u} \delta \dot{u}+\dot{v} \delta \dot{v}+\dot{w} \delta \dot{w}] d V
$$

where $\rho$ is the mass density and $\left(^{*}\right)$ represents partial derivative with respect to time.

\subsubsection{Virtual Kinetic energy of a composite beam using CLPT:}

The virtual kinetic energy for the partial plane stress using CLPT is obtained by substituting the displacement field for CLPT in Eq. (3.85) as shown below

$$
\begin{aligned}
& \delta T=\iiint_{V} \rho\left[\left(\dot{u}_{o}-z \dot{w}^{\prime}\right)\left(\delta \dot{u}_{o}-z \delta \dot{w}^{\prime}\right)\right. \\
&\left.+\left(\dot{v}_{o}-z \dot{w}^{y}\right)\left(\delta \dot{v}_{o}-z \delta \dot{w}^{y}\right)+\dot{w} \delta \dot{w}\right] d V
\end{aligned}
$$

Expanding the terms inside the integral 


$$
\begin{array}{r}
\delta T=\iiint_{V} \rho\left(\dot{u}_{o} \delta \dot{u}_{o}-z \dot{u}_{o} \delta \dot{w}^{\prime}-z \dot{w}^{\prime} \delta \dot{u}_{o}+z^{2} \dot{w}^{\prime} \delta \dot{w}^{\prime}+\dot{v}_{o} \delta \dot{v}_{o}\right. \\
\left.-z \dot{v}_{o} \delta \dot{w}^{y}-z \dot{w}^{y} \delta \dot{v}_{o}+z^{2} \dot{w}^{y} \delta \dot{w}^{y}+\dot{w} \delta \dot{w}\right) d V
\end{array}
$$

$\delta T$ has a time integral when it is introduced in Hamilton's principle and Using partial plane stress approximation to reduce to one dimensional problem Eq. (3.87) becomes

$$
\begin{gathered}
\int_{t_{1}}^{t_{2}} \delta T=\int_{t_{1}}^{t_{2}} d t \iiint_{V} \rho\left(\dot{u}_{o} \delta \dot{u}_{o}-z \dot{u}_{o} \delta \dot{w}^{\prime}-z \dot{w}^{\prime} \delta \dot{u}_{o}+z^{2} \dot{w}^{\prime} \delta \dot{w}^{\prime}\right. \\
\left.+z^{2} \dot{w}^{y} \delta \dot{w}^{y}+\dot{w} \delta \dot{w}\right) d V
\end{gathered}
$$

Integrating by parts with respect to the time $t$ and denoting the boundary terms as $(\ldots)_{t_{1}}^{t_{2}}$,

$$
\begin{gathered}
-\int_{t_{1}}^{t_{2}} \delta T=\iiint_{V} \rho \int_{t_{1}}^{t_{2}}\left\{\left[\ddot{u}_{o} \delta u_{o}-z \ddot{u}_{o} \delta w^{\prime}+z^{2} \ddot{w}^{\prime} \delta w^{\prime}-z \ddot{w}^{\prime} \delta u_{o}+z^{2} \ddot{w}^{y} \delta w^{y}\right.\right. \\
\left.+\ddot{w} \delta w] d t+(\ldots)_{t_{1}}^{t_{2}}\right\} d V
\end{gathered}
$$

Neglect the boundary terms as they do not contribute to the inertia matrix. As the variational quantities do not have the time derivatives, they can be taken out of the time integral.

Therefore

$$
\begin{gathered}
-\delta T=\iiint_{V} \rho\left(\ddot{u}_{o} \delta u_{o}-z \ddot{u}_{o} \delta w^{\prime}+z^{2} \ddot{w}^{\prime} \delta w^{\prime}+z^{2} \ddot{w}^{y} \delta w^{y}\right. \\
\left.-z \ddot{w}^{\prime} \delta u_{o}+\ddot{w} \delta w\right) d V
\end{gathered}
$$

Splitting the volume integral into integral over thickness, length and width

$$
\begin{gathered}
-\delta T=b \int_{0}^{L} \int_{-h / 2}^{h / 2} \rho\left(\ddot{u}_{o} \delta u_{o}-z \ddot{u}_{o} \delta w^{\prime}+z^{2} \ddot{w}^{\prime} \delta w^{\prime}+z^{2} \ddot{w}^{y} \delta w^{y}\right. \\
\left.-z \ddot{w}^{\prime} \delta u_{o}+\ddot{w} \delta w\right) d z d x
\end{gathered}
$$


Expressing Eq. (3.91) in terms inertia coefficients, the virtual kinetic energy for the composite beam reduces to

$$
\begin{gathered}
-\delta T=b \int_{0}^{L}\left(I_{0} \ddot{u}_{o} \delta u_{o}-I_{1} \ddot{u}_{o} \delta w^{\prime}+I_{2} \ddot{w}^{\prime} \delta w^{\prime}+I_{2} \ddot{w}^{y} \delta w^{y}\right. \\
\left.-I_{1} \ddot{w}^{\prime} \delta u_{o}+I_{0} \ddot{w} \delta w\right) d x
\end{gathered}
$$

Where

$$
\begin{gathered}
I_{0}=\int_{-h / 2}^{h / 2} \rho d z \\
I_{1}=\int_{-h / 2}^{h / 2} \rho z d z \\
I_{2}=\int_{-h / 2}^{h / 2} \rho z^{2} d z
\end{gathered}
$$

\subsubsection{Virtual Kinetic energy of a composite beam for using FSDT:}

The virtual kinetic energy using FSDT is obtained by substituting the displacement field for FSDT in Eq. (3.85) as shown below

$$
\begin{gathered}
\delta T=\iiint_{V} \rho\left[\left(\dot{u}_{o}-z \dot{w}_{b}^{\prime}\right)\left(\delta \dot{u}_{o}-z \delta \dot{w}_{b}^{\prime}\right)+\left(\dot{v}_{o}-z \dot{w}_{b}^{y}\right)\left(\delta \dot{v}_{o}-z \delta \dot{w}_{b}^{y}\right)\right. \\
\left.+\left(\dot{w}_{b}+\dot{w}_{s}\right)\left(\delta \dot{w}_{b}+\delta \dot{w}_{s}\right)\right] d V
\end{gathered}
$$

Expanding the terms inside the integral 


$$
\begin{aligned}
\iiint_{V} \rho\left(\dot{u}_{o} \delta \dot{u}_{o}\right. & -z \dot{u}_{o} \delta \dot{w}_{b}^{\prime}-z \dot{w}_{b}^{\prime} \delta \dot{u}_{o}+z^{2} \dot{w}_{b}^{\prime} \delta \dot{w}_{b}^{\prime}+\dot{v}_{o} \delta \dot{v}_{o}-z \dot{v}_{o} \delta \dot{w}_{b}^{y} \\
& -z \dot{w}_{b}^{y} \delta \dot{v}_{o}+z^{2} \dot{w}_{b}^{y} \delta \dot{w}_{b}^{y}+\dot{w}_{b} \delta \dot{w}_{b}+\dot{w}_{b} \delta \dot{w}_{s}+\dot{w}_{s} \delta \dot{w}_{b} \\
& \left.+\dot{w}_{s} \delta \dot{w}_{s}\right) d V
\end{aligned}
$$

$\delta T$ has a time integral when it is introduced in Hamilton's principle and reducing to one dimensional problem, Eq. (3.95) becomes

$$
\begin{gathered}
\int_{t_{1}}^{t_{2}} \delta T=\int_{t_{1}}^{t_{2}} d t \\
\iiint_{V} \rho\left(\dot{u}_{o} \delta \dot{u}_{o}-z \dot{u}_{o} \delta \dot{w}_{b}^{\prime}-z \dot{w}_{b}^{\prime} \delta \dot{u}_{o}+z^{2} \dot{w}_{b}^{\prime} \delta \dot{w}_{b}^{\prime}\right. \\
+z^{2} \dot{w}_{b}^{y} \delta \dot{w}_{b}^{y}+\dot{w}_{b} \delta \dot{w}_{b}+\dot{w}_{b} \delta \dot{w}_{s}+\dot{w}_{s} \delta \dot{w}_{b} \\
\left.+\dot{w}_{S} \delta \dot{w}_{s}\right) d V
\end{gathered}
$$

Integrating by parts with respect to the time cordinate $t$ and denoting the boundary terms as $(\ldots)_{t_{1}}^{t_{2}}$,

$$
\begin{aligned}
-\int_{t_{1}}^{t_{2}} \delta T=\iiint_{V} \rho \int_{t_{1}}^{t_{2}}\left\{\left[\ddot{u}_{o} \delta u_{o}-z \ddot{u}_{o} \delta w_{b}^{\prime}+z^{2} \ddot{w}_{b}^{\prime} \delta w_{b}^{\prime}-z \ddot{w}_{b}^{\prime} \delta u_{o}\right.\right. \\
\left.+z^{2} \ddot{w}_{b}^{y} \delta w_{b}^{y}+\ddot{w}_{b} \delta w_{b}+\ddot{w}_{b} \delta w_{s}+\ddot{w}_{s} \delta w_{s}+\ddot{w}_{s} \delta w_{b}\right] d t \\
\left.-(\ldots)_{t_{1}}^{t_{2}}\right\} d V
\end{aligned}
$$

Neglect the boundary terms as they do not contribute to the inertia matrix. As the variational quantities do not have the time derivatives, they can be taken out of the time integral. Therefore 


$$
\begin{aligned}
-\delta T=\iiint_{V} \rho\left(\ddot{u}_{o} \delta u_{o}-z \ddot{u}_{o} \delta w_{b}^{\prime}+z^{2} \ddot{w}_{b}^{\prime} \delta w_{b}^{\prime}+z^{2} \ddot{w}_{b}^{y} \delta w_{b}^{y}\right. \\
-z \ddot{w}_{b}^{\prime} \delta u_{o}+\ddot{w}_{b} \delta w_{b}+\ddot{w}_{b} \delta w_{s}+\ddot{w}_{s} \delta w_{b} \\
\left.+\ddot{w}_{S} \delta w_{s}\right) d V
\end{aligned}
$$

Splitting the volume integral into integral over thickness, length and width

$$
\begin{gathered}
-\delta T=b \int_{-h / 2}^{h / 2} \int_{0}^{l} \rho\left(\ddot{u}_{o} \delta u_{o}-z \ddot{u}_{o} \delta w_{b}^{\prime}+z^{2} \ddot{w}_{b}^{\prime} \delta w_{b}^{\prime}+z^{2} \ddot{w}_{b}^{y} \delta w_{b}^{y}\right. \\
-z \ddot{w}_{b}^{\prime} \delta u_{o}+\ddot{w}_{b} \delta w_{b}+\ddot{w}_{b} \delta w_{s}+\ddot{w}_{s} \delta w_{b} \\
\left.+\ddot{w}_{s} \delta w_{s}\right) d z d x
\end{gathered}
$$

Expressing Eq. (3.99) in terms inertia coefficients, the virtual kinetic energy for the composite beam reduces to

$$
\begin{aligned}
-\delta T=b \int_{0}^{l} I_{0} \ddot{u}_{o} \delta u_{o}-I_{1} \ddot{u}_{o} \delta w_{b}^{\prime}+I_{2} \ddot{w}_{b}^{\prime} \delta w_{b}^{\prime}+I_{2} \ddot{w}_{b}^{y} \delta w_{b}^{y} \\
-I_{1} \ddot{w}_{b}^{\prime} \delta u_{o}+I_{0} \ddot{w}_{b} \delta w_{b}+I_{0} \ddot{w_{b}} \delta w_{s}+I_{0} \ddot{w}_{s} \delta w_{b} \\
+I_{0} \ddot{w}_{s} \delta w_{s} d x
\end{aligned}
$$

$I_{i}=\int_{-h / 2}^{h / 2} \rho z^{i} d z$ for $i=0,1,2$

where $I_{0}, I_{1}, I_{2}$ represent normal, coupled normal-rotary, rotary inertia coefficients.

\subsubsection{Virtual kinetic energy of a composite beam for partial plane stress using}

\section{HSDT}

The virtual kinetic energy for the partial plane stress using HSDT is obtained by substituting the displacement field for HSDT in Eq. (3.85) as shown below 


$$
\begin{aligned}
\delta T=\iiint_{V} \rho\left[\left(\dot{u}_{o}-z \dot{w}_{b}^{\prime}-c_{1} z^{3} \dot{w}_{s}^{\prime}\right)\left(\delta \dot{u}_{o}-z \delta \dot{w_{b}}-c_{1} z^{3} \delta \dot{w}_{s}^{\prime}\right)\right. \\
+\left(\dot{v}_{o}-z \dot{w}_{b}^{y}-c_{1} z^{3} \dot{w}_{s}^{y}\right)\left(\delta \dot{v}_{o}-z \delta \dot{w}_{b}^{y}-c_{1} z^{3} \delta \dot{w}_{s}^{y}\right) \\
\left.+\left(\dot{w}_{b}+\dot{w}_{s}\right)\left(\delta \dot{w}_{b}+\delta \dot{w}_{s}\right)\right] d V
\end{aligned}
$$

On neglecting $\dot{v}_{o}$ and $\delta \dot{v}_{o}$, Eq. (3.101) reduces to one dimensional formulation

$$
\begin{aligned}
& \delta T=\iiint_{V} \rho\left[\left(\dot{u}_{o}-z \dot{w}_{b}^{\prime}-c_{1} z^{3} \dot{w}_{s}^{\prime}\right) \delta \dot{u}_{o}\right. \\
&-\left(z \dot{u}_{o}-z^{2} \dot{w}_{b}^{\prime}-c_{1} z^{4} \dot{w}_{s}^{\prime}\right) \delta \dot{w}_{b}^{\prime} \\
&-c_{1}\left(z^{3} \dot{u}_{o}-z^{4} \dot{w}_{b}^{\prime}-c_{1} z^{6} \dot{w}_{s}^{\prime}\right) \delta \dot{w}_{s}^{\prime} \\
&+\left(z^{2} \dot{w}_{b}^{y}+c_{1} z^{4} \dot{w}_{s}^{y}\right) \delta \dot{w}_{b}^{y} \\
&+c_{1}\left(z^{4} \dot{w}_{b}^{y}+c_{1} z^{6} \dot{w}_{s}^{y}\right) \delta \dot{w}_{s}^{y}+\left(\dot{w}_{b}+\dot{w}_{s}\right) \delta \dot{w}_{b} \\
&\left.+\left(\dot{w}_{b}+\dot{w}_{s}\right) \delta \dot{w}_{s}\right] d V
\end{aligned}
$$

Applying the time integral and integrating by parts and denoting the boundary terms as $(\ldots)_{t_{1}}^{t_{2}}$

$$
\begin{aligned}
-\int_{t_{1}}^{t_{2}} \delta T d t= & \iiint_{V}\left\{\int _ { t _ { 1 } } ^ { t _ { 2 } } \rho \left[\left(\ddot{u}_{o}-z \ddot{w}_{b}^{\prime}-c_{1} z^{3} \ddot{w}_{s}^{\prime}\right) \delta u\right.\right. \\
& -\left(z \ddot{u}_{o}-z^{2} \dot{w}_{b}^{\prime}-c_{1} z^{4} \dot{w}_{s}^{\prime}\right) \delta w_{b}^{\prime} \\
& -c_{1}\left(z^{3} \ddot{u}_{o}-z^{4} \ddot{w}_{b}^{\prime}-c_{1} z^{6} \ddot{w}_{s}^{\prime}\right) \delta w_{s}^{\prime} \\
& +\left(z^{2} \ddot{w}_{b}^{y}+c_{1} z^{4} \ddot{w}_{s}^{y}\right) \delta w_{b}^{y} \\
& +c_{1}\left(z^{4} \ddot{w}_{b}^{y}+c_{1} z^{6} \ddot{w}_{s}^{y}\right) \delta w_{s}^{y}+\left(\ddot{w}_{b}+\ddot{w}_{s}\right) \delta w_{b} \\
& \left.\left.+\left(\ddot{w}_{b}+\ddot{w}_{s}\right) \delta w_{s}\right] d t+(\ldots)_{t_{1}}^{t_{2}}\right\} d V
\end{aligned}
$$

Neglect the boundary terms as they do not contribute to the inertia matrix. As the variational quantities do not have the time derivatives, they can be taken out of the time integral. 
Therefore,

$$
\begin{aligned}
-\delta T=\iiint_{V} \rho[ & \left(\ddot{u}_{o}-z \ddot{w}_{b}^{\prime}-c_{1} z^{3} \ddot{w}_{s}^{\prime}\right) \delta u \\
& -\left(z \ddot{u}_{o}-z^{2} \dot{w}_{b}^{\prime}-c_{1} z^{4} \dot{w}_{s}^{\prime}\right) \delta w_{b}^{\prime} \\
& -c_{1}\left(z^{3} \ddot{u}_{o}-z^{4} \ddot{w}_{b}^{\prime}-c_{1} z^{6} \ddot{w}_{s}^{\prime}\right) \delta w_{s}^{\prime} \\
& +\left(z^{2} \ddot{w}_{b}^{y}+c_{1} z^{4} \ddot{w}_{s}^{y}\right) \delta w_{b}^{y} \\
& +c_{1}\left(z^{4} \ddot{w}_{b}^{y}+c_{1} z^{6} \ddot{w}_{s}^{y}\right) \delta w_{s}^{y}+\left(\ddot{w}_{b}+\ddot{w}_{s}\right) \delta w_{b} \\
& \left.+\left(\ddot{w}_{b}+\ddot{w}_{s}\right) \delta w_{s}\right] d V
\end{aligned}
$$

Splitting the volume integral into integral over thickness, length and width

$$
\begin{aligned}
-\delta T=b \int_{0}^{L} \int_{-h / 2}^{h / 2} \rho\left[\left(\ddot{u}_{o}-z \ddot{w}_{b}^{\prime}-c_{1} z^{3} \ddot{w}_{s}^{\prime}\right) \delta u\right. \\
\quad-\left(z \ddot{u}_{o}-z^{2} \dot{w}_{b}^{\prime}-c_{1} z^{4} \dot{w}_{s}^{\prime}\right) \delta w_{b}^{\prime} \\
\quad-c_{1}\left(z^{3} \ddot{u}_{o}-z^{4} \ddot{w}_{b}^{\prime}-c_{1} z^{6} \ddot{w}_{s}^{\prime}\right) \delta w_{s}^{\prime} \\
+\left(z^{2} \ddot{w}_{b}^{y}+c_{1} z^{4} \ddot{w}_{s}^{y}\right) \delta w_{b}^{y} \\
+c_{1}\left(z^{4} \ddot{w}_{b}^{y}+c_{1} z^{6} \ddot{w}_{s}^{y}\right) \delta w_{s}^{y}+\left(\ddot{w}_{b}+\ddot{w}_{s}\right) \delta w_{b} \\
\left.+\left(\ddot{w}_{b}+\ddot{w}_{s}\right) \delta w_{s}\right] d z d x
\end{aligned}
$$

Eq. (3.105) can be reduced further by introducing the inertia coefficients,

$I_{i}=\int_{-h / 2}^{h / 2} \rho z^{i} d z$ for $i=0,1,2,3,4,5,6$

where $I_{0}, I_{1}, I_{2}$ represent normal, coupled normal-rotary, rotary inertia coefficients and others represent higher-order inertia coefficients. 


\subsection{Virtual work done on a composite plate}

The work is done on the composite plate due to mechanical and electrical forces. If $T$ is the surface traction in the $x_{i}$ direction, $F_{q}$ is the electric charge applied on the surface $\mathrm{S}$. The work done on the structure is given by

$$
W_{e}=\iint_{S}\left(T u_{i}-F_{q} \varphi\right) d S
$$

The virtual work done is given by

$$
\delta W_{e}=\iint_{S}\left(T_{i} \delta u_{i}-F_{q} \delta \varphi\right) d S
$$

In the present research no external forces act on the system. But the work done due to electrical charge is kept and used for deriving the actuator equations. 


\section{FINITE ELEMENT FORMULATION}

\subsection{Introduction}

Many engineering problems are represented mathematically by partial differential equations. It is not possible to obtain closed form solution in all situations; Numerical methods are used to arrive at approximate solutions in such cases. The closed form solution is valid at all points in a domain whereas the numerical methods give approximate solution at discrete points in the domain. The finite element analysis is one of the numerical methods for solving elliptical partial differential equations wherein the domain is discretized into finite elements. The finite element solution approaches exact solution with increasing the number of elements or increasing number of internal nodes or both. Based on this three types of finite element formulations are available, namely $h$-version, $p$-version, $h-p$ version. In the h-version the accuracy increases with increasing number of elements, in general. In p-version, the number of internal nodes are increased which in turn vary the order of the interpolation functions; In general higher the order of polynomial, higher is the accuracy. In the h-p version, both the number of elements and internal nodes are varied to increase the accuracy. In the present case, the h-p version is used to discretize the beam. Sreeram and Sivaneri (1997) conducted a convergence study on an isotropic moving beam problem and concluded that four elements with three internal nodes each produce an accurate solution. In the present research, the composite moving beam is divided into four elements with three internal nodes each [19], [20]. 


\subsection{Finite element shape functions}

The composite beam considered here is divided into 4 elements with 3 internal nodes each. Certain degrees of freedom for this beam obey $\mathrm{C}^{0}$ continuity while others satisfy $\mathrm{C}^{1}$ continuity; the corresponding shape functions are derived using Lagrangian and Hermitian polynomials, respectively. For the variables that satisfy $\mathrm{C}^{1}$ continuity, slope degrees of freedom are assumed only at the end nodes as the slope continuity is automatically assured at the internal nodes.

A natural coordinate $(\xi=[-1,1])$ is introduced with its origin at the center of the element. The transformation between the element coordinate $\left(x_{e}\right)$ and natural coordinate $(\xi)$ system is given by,

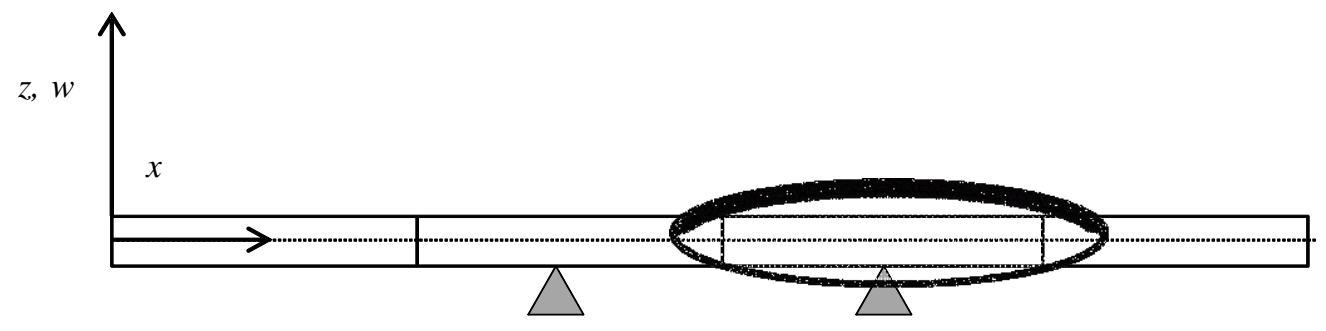

Figure 4.1 Finite element representation of a moving beam

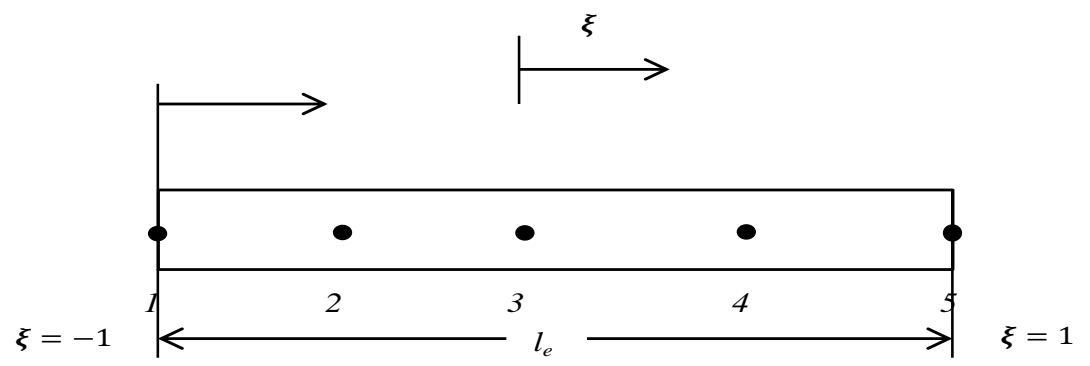

Figure 4.2 Representation of natural coordinate system of a finite element with three internal nodes 


$$
\begin{aligned}
& x_{e}=\frac{l_{e}}{2}(\xi+1) \\
& d x_{e}=\frac{l_{e}}{2} d \xi
\end{aligned}
$$

where $l_{e}$ is the length of the element. Depending on the composite plate theory used (CLPT, FSDT, or HSDT), the element variables differ in number and as indicated earlier, some of these variables follow $\mathrm{C}^{0}$ continuity and the rest $\mathrm{C}^{1}$ continuity. In the following the shape functions for $\mathrm{C}^{0}$ and $\mathrm{C}^{1}$ continuity are derived by taking one example $\left(u_{\mathrm{e}}\right)$ for $\mathrm{C}^{0}$ continuity and one example $(w)$ for $\mathrm{C}^{1}$ continuity. The axial, $u(\xi)$ and transverse, $w(\xi)$ degrees of freedom are represented as follows

$$
\begin{gathered}
u(\xi)=\sum_{i=0}^{4} a_{i} \xi^{i} \\
w(\xi)=\sum_{j=0}^{6} b_{j} \xi^{j}
\end{gathered}
$$

The above equations in matrix form are written as

$$
\begin{aligned}
& u(\xi)=\left\lfloor\xi^{i}\right\rfloor\left\{a_{i}\right\} \\
& w(\xi)=\left\lfloor\xi^{j}\right\rfloor\left\{b_{j}\right\}
\end{aligned}
$$

where $a_{i}$ and $b_{j}$ are to be determined.

To solve for $a_{i} s$ in Eq. (4.2), five equations are required and the axial degrees of freedom at the nodes of an element are considered. 


$$
\begin{array}{r}
u(-1)=u_{1} \\
u(-0.5)=u_{2} \\
u(0)=u_{3} \\
u(0.5)=u_{4} \\
u(1)=u_{5}
\end{array}
$$

On solving the above equations, $u(\xi)$ can be interpolated in terms of Lagrange polynomials Therefore,

$$
u(\xi)=\left\lfloor\begin{array}{lll}
H_{L 1} & \cdots & H_{L 5}
\end{array}\right\rfloor\left\{\begin{array}{c}
u_{1} \\
\vdots \\
u_{5}
\end{array}\right\}
$$

where $H_{L 1}(\xi), H_{L 2}(\xi)$, etc., are called Legrangian shape functions and are given by,

$$
\begin{gathered}
H_{L 1}=\frac{1}{6} \xi-\frac{1}{6} \xi^{2}-\frac{2}{3} \xi^{3}+\frac{2}{3} \xi^{4} \\
H_{L 2}=-\frac{4}{3} \xi+\frac{8}{3} \xi^{2}+\frac{4}{3} \xi^{3}-\frac{8}{3} \xi^{4} \\
H_{L 3}=1-5 \xi^{2}+4 \xi^{4} \\
H_{L 4}=\frac{4}{3} \xi+\frac{8}{3} \xi^{2}-\frac{4}{3} \xi^{3}-\frac{8}{3} \xi^{4} \\
H_{L 5}=-\frac{1}{6} \xi-\frac{1}{6} \xi^{2}+\frac{2}{3} \xi^{3}+\frac{2}{3} \xi^{4}
\end{gathered}
$$

Similarly, to solve for $b_{j} s$, in Eq. (4.3) seven equations are required and the transverse degrees of freedom at the nodes of an element are considered

$$
\begin{gathered}
w(-1)=w_{1} \\
\frac{l_{e}}{2} w^{\prime}(-1)=w_{1}{ }^{\prime} \\
w(-0.5)=w_{2}
\end{gathered}
$$




$$
\begin{gathered}
w(0)=w_{3} \\
w(0.5)=w_{4} \\
w(1)=w_{5} \\
\frac{l_{e}}{2} w^{\prime}(1)=w_{5}{ }^{\prime}
\end{gathered}
$$

On solving the above equations, $w(\xi)$ can be interpolated in terms of Hermite polynomials.

Therefore,

$$
w(\xi)=\left\lfloor\begin{array}{llll}
H_{1} & \cdots & \cdots & H_{7}
\end{array}\right\rfloor\left\{\begin{array}{c}
w_{1} \\
w_{1}^{\prime} \\
\vdots \\
w_{5} \\
w_{5}^{\prime}
\end{array}\right\}
$$

where $H_{1}(\xi), H_{2}(\xi)$, etc., are called Hermite shape functions and are given by

$$
\begin{gathered}
H_{1}=\frac{1}{9}\left(\frac{17}{4} \xi-5 \xi^{2}-\frac{79}{4} \xi^{3}+\frac{47}{2} \xi^{4}+11 \xi^{5}-14 \xi^{6}\right) \\
H_{2}=\frac{l_{e}}{6}\left(\frac{1}{4} \xi-\frac{1}{4} \xi^{2}-\frac{5}{4} \xi^{3}+\frac{5}{4} \xi^{4}+\xi^{5}-\xi^{6}\right) \\
H_{3}=\frac{16}{9}\left(-\xi+2 \xi^{2}+2 \xi^{3}-4 \xi^{4}-\xi^{5}+2 \xi^{6}\right) \\
H_{5}=\frac{16}{9}\left(\xi+2 \xi^{2}-2 \xi^{3}-4 \xi^{4}+\xi^{5}+2 \xi^{6}\right) \\
H_{6}=\frac{1}{9}\left(-\frac{17}{4} \xi-5 \xi^{2}+\frac{79}{4} \xi^{3}+\frac{47}{2} \xi^{4}-11 \xi^{5}-14 \xi^{6}\right) \\
H_{7}=\frac{l_{e}}{6}\left(\frac{1}{4} \xi+\frac{1}{4} \xi^{2}-\frac{5}{4} \xi^{3}-\frac{5}{4} \xi^{4}+\xi^{5}+\xi^{6}\right)
\end{gathered}
$$

\subsection{Element stiffness matrix formulation}

\subsubsection{Element Stiffness matrix for beam using CLPT}

The beam element using CLPT has the following degrees of freedom

At end nodes: $u, \gamma, w, w^{\prime}, w^{y}$ 
At internal nodes: $u, \gamma, w, w^{y}$

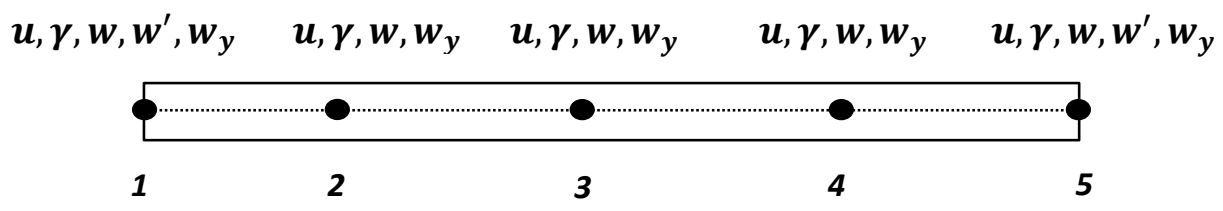

Figure 4.3 Element definition for reduced beam formulation using CLPT

The element has a total of 22 degrees of freedom. $u, \gamma$ and $w_{y}$ assume $C^{0}$ continuity and are represented by Lagrange shape functions. While the variable $w$ assumes $C^{1}$ continuity and is represented by Hermite shape functions.

$$
\begin{gathered}
u=\left\lfloor H_{L}\right\rfloor\left\{q_{u}\right\} \\
\gamma=\left\lfloor H_{L}\right\rfloor\left\{q_{\gamma}\right\} \\
w_{y}=\left\lfloor H_{L}\right\rfloor\left\{q_{w_{y}}\right\} \\
w=\lfloor H\rfloor\left\{q_{w}\right\}
\end{gathered}
$$

The stiffness matrix is obtained from the virtual mechanical strain energy equation Eq. (3.52) and the virtual electrical strain energy Eq. (3.80).

The virtual forms of the variables are,

$$
\begin{gathered}
\delta u=\left\lfloor\delta q_{u}\right\rfloor\left\{H_{L}\right\} \\
\delta \gamma=\left\lfloor\delta q_{\gamma}\right\rfloor\left\{H_{L}\right\} \\
\delta w_{y}=\left\lfloor\delta q_{w_{y}}\right\rfloor\left\{H_{L}\right\} \\
\delta w=\left\lfloor\delta q_{w}\right\rfloor\{H\}
\end{gathered}
$$

Substituting the above equations in the virtual mechanical strain energy equation and electrical strain energy expressions, we get, 


$$
\delta U=\left[\delta q_{e}\right]\left[K_{e}\right]\left\{q_{e}\right\}
$$

The stiffness matrix is formed by 36 sub matrices is obtained. The sub matrices for the elastic part are $\quad\left[K_{u u}\right],\left[K_{\gamma \gamma}\right],\left[K_{w w}\right],\left[K_{w_{y} w_{y}}\right],\left[K_{u \gamma}\right],\left[K_{u w}\right],\left[K_{u w_{y}}\right],\left[K_{\gamma w}\right],\left[K_{\gamma w_{y}}\right],\left[K_{w w_{y}}\right]$ with dimensions $5 \times 5,5 \times 5,7 \times 7,5 \times 5,5 \times 5,5 \times 7,5 \times 5,5 \times 7,5 \times 5$ and $7 \times 5$ each. The sub matrices for the piezoelectric sensor and actuator part are represented as $\left[K_{u \varphi_{a}}\right],\left[K_{\gamma \varphi_{a}}\right],\left[K_{w \varphi_{a}}\right],\left[K_{w^{y} \varphi_{a}}\right]$, $\left[K_{u \varphi_{s}}\right],\left[K_{\gamma \varphi_{s}}\right],\left[K_{w \varphi_{s}}\right],\left[K_{w^{y} \varphi_{s}}\right]$ with dimensions $5 \times 1,5 \times 1,7 \times 1,5 \times 1,5 \times 1,5 \times 1,7 \times 1$ and $5 \times 1$ respectively. The sub matrices for the electrical part are represented as $\left[K_{\varphi_{a} \varphi_{a}}\right]$ and $\left[K_{\varphi_{s} \varphi_{s}}\right]$ with dimensions $1 \times 1$ and $1 \times 1$ respectively.

The sub matrices are given by

$$
\begin{aligned}
& {\left[K_{u u}\right] }=b \int_{0}^{l_{e}} \bar{S}_{11}\left\{H_{L}^{\prime}\right\}\left\lfloor H_{L}^{\prime}\right\rfloor d x \\
& {\left[K_{u \gamma}\right] }=b \int_{0}^{l_{e}} \bar{S}_{12}\left\{H_{L}^{\prime}\right\}\left\lfloor H_{L}\right\rfloor d x \\
& {\left[K_{u w}\right] }=-b \int_{0}^{l_{e}} \bar{S}_{13}\left\{H_{L}^{\prime}\right\}\left\lfloor H^{\prime \prime}\right\rfloor d x \\
& {\left[K_{u w_{y}}\right] }=-2 b \int_{0}^{l_{e}} \bar{S}_{14}\left\{H_{L}^{\prime}\right\}\left\lfloor H_{L}^{\prime}\right\rfloor d x \\
& {\left[K_{\gamma \gamma}\right] }=b \int_{0}^{l_{e}} \bar{S}_{22}\left\{H_{L}\right\}\left\lfloor H_{L}\right\rfloor d x \\
& {\left[K_{\gamma w}\right] }=b \int_{0}^{l_{e}} \bar{S}_{23}\left\{H_{L}\right\}\left\lfloor H^{\prime \prime}\right\rfloor d x \\
& {\left[K_{\gamma w_{y}}\right] }=-2 b \int_{0}^{l_{e}} \bar{S}_{24}\left\{H_{L}\right\}\left\lfloor H_{L}^{\prime}\right\rfloor d x \\
& {\left[K_{w w}\right]=b \int_{0}^{l_{e}} \bar{S}_{33}\left\{H^{\prime \prime}\right\}\left\lfloor H^{\prime \prime}\right\rfloor d x+b \int_{0}^{l_{e}} N_{x}\left\{H^{\prime}\right\}\left\lfloor H^{\prime}\right\rfloor d x }
\end{aligned}
$$




$$
\begin{aligned}
& {\left[K_{w w_{y}}\right]=2 b \int_{0}^{l_{e}} \bar{S}_{34}\left\{H^{\prime \prime}\right\}\left[H_{L}^{\prime}\right\rfloor d x} \\
& {\left[K_{w w_{y}}\right]=2 b \int_{0}^{l_{e}} \bar{S}_{34}\left\{H^{\prime \prime}\right\}\left\lfloor H_{L}^{\prime}\right\rfloor d x} \\
& {\left[K_{w_{y} w_{y}}\right]=4 b \int_{0}^{l_{e}} \bar{S}_{34}\left\{H_{L}^{\prime}\right\}\left\lfloor H_{L}^{\prime}\right\rfloor d x} \\
& {\left[K_{u \varphi_{a}}\right]=b \int_{0}^{l_{e}} S_{P 1 a}\left\{H_{L}^{\prime}\right\} d x} \\
& {\left[K_{\gamma \varphi_{a}}\right]=b \int_{0}^{l_{e}} S_{P 2 a}\left\{H_{L}\right\} d x} \\
& {\left[K_{w \varphi_{a}}\right]=-b \int_{0}^{l_{e}} S_{P 3 a}\left\{H^{\prime \prime}\right\} d x} \\
& {\left[K_{w_{y} \varphi_{a}}\right]=-2 b \int_{0}^{l_{e}} S_{P 4 a}\left\{H_{L}^{\prime}\right\} d x} \\
& {\left[K_{u \varphi_{s}}\right]=b \int_{0}^{l_{e}} S_{P 1 s}\left\{H_{L}^{\prime}\right\} d x} \\
& {\left[K_{\gamma \varphi_{s}}\right]=b \int_{0}^{l_{e}} S_{P 2 s}\left\{H_{L}\right\} d x} \\
& {\left[K_{w \varphi_{s}}\right]=-b \int_{0}^{l_{e}} S_{P 3 s}\left\{H^{\prime \prime}\right\} d x} \\
& {\left[K_{w_{y} \varphi_{s}}\right]=-2 b \int_{0}^{l_{e}} S_{P 4 s}\left\{H_{L}^{\prime}\right\} d x} \\
& {\left[K_{\varphi_{a} \varphi_{a}}\right]=-\frac{b}{t_{k}} \int_{0}^{l_{e}}\left(\epsilon_{33}\right)_{a} d x-\frac{b}{t_{k}} \int_{0}^{l_{e}} D_{\varphi_{a}} d x} \\
& {\left[K_{\varphi_{s} \varphi_{s}}\right]=-\frac{b}{t_{k}} \int_{0}^{l_{e}}\left(\epsilon_{33}\right)_{s} d x-\frac{b}{t_{k}} \int_{0}^{l_{e}} D_{\varphi_{s}} d x}
\end{aligned}
$$


The complete element stiffness matrix takes the form

$$
\left[K_{e}\right]=\left[\begin{array}{cccccc}
{\left[K_{u u}\right]} & {\left[K_{u \gamma}\right]} & {\left[K_{u w}\right]} & {\left[K_{u w_{y}}\right]} & {\left[K_{u \varphi_{a}}\right]} & {\left[K_{u \varphi_{s}}\right]} \\
{\left[K_{u \gamma}\right]^{T}} & {\left[K_{\gamma \gamma}\right]} & {\left[K_{\gamma w}\right]} & {\left[K_{\gamma w_{y}}\right]} & {\left[K_{\gamma \varphi_{a}}\right]} & {\left[K_{\gamma \varphi_{s}}\right]} \\
{\left[K_{u w}\right]^{T}} & {\left[K_{\gamma w}\right]^{T}} & {\left[K_{w w}\right]} & {\left[K_{w w_{y}}\right]} & {\left[K_{w \varphi_{a}}\right]} & {\left[K_{w \varphi_{s}}\right]} \\
{\left[K_{u w_{y}}\right]^{T}} & {\left[K_{\gamma w_{y}}\right]^{T}} & {\left[K_{w w_{y}}\right]^{T}} & {\left[K_{w_{y} w_{y}}\right]} & {\left[K_{w_{y} \varphi_{a}}\right]} & {\left[K_{w_{y} \varphi_{s}}\right]} \\
{\left[K_{u \varphi_{a}}\right]^{T}} & {\left[K_{\gamma \varphi_{a}}\right]^{T}} & {\left[K_{w \varphi_{a}}\right]^{T}} & {\left[K_{w_{y} \varphi_{a}}\right]^{T}} & {\left[K_{\varphi_{a} \varphi_{a}}\right]} & {\left[K_{\varphi_{a} \varphi_{s}}\right]} \\
{\left[K_{u \varphi_{s}}\right]^{T}} & {\left[K_{\gamma \varphi_{s}}\right]^{T}} & {\left[K_{w \varphi_{s}}\right]^{T}} & {\left[K_{w_{y} \varphi_{s}}\right]^{T}} & {\left[K_{\varphi_{s} \varphi_{a}}\right]} & {\left[K_{\varphi_{s} \varphi_{s}}\right]}
\end{array}\right]
$$

The element stiffness matrix can be written as

$$
\left[K_{e}\right]=\left[\begin{array}{cc}
{\left[K_{s s}\right]} & {\left[K_{s \varphi}\right]} \\
{\left[K_{s \varphi}\right]^{T}} & {\left[K_{\varphi \varphi}\right]}
\end{array}\right]
$$

where $\left[K_{S S}\right],\left[K_{S \varphi}\right]$ and $\left[K_{\varphi \varphi}\right]$ represent the elastic, piezoelectric and electric stiffness matrices.

\subsubsection{Element Stiffness Matrix for beam using FSDT}

The beam element using FSDT has the following degrees of freedom

At the end nodes: $u, \gamma, w_{b}, w_{b}^{\prime}, w_{s}, w_{s}^{\prime}, w_{b}^{y}$

At the mid side nodes: $u, \gamma, w_{b}, w_{s}, w_{b}^{y}$

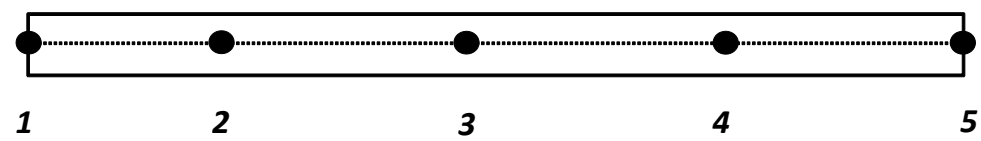

For nodes $(1,5): u, \gamma, w_{b}, w_{b}^{\prime}, w_{s}, w_{s}^{\prime}, w_{b}^{y}$

For nodes $(2,3,4): u, \gamma, w_{b}, w_{s}, w_{b}^{y}$

Figure 4.4 Element definition for reduced beam formulation using FSDT 
The element has 29 degrees of freedom. The variables $u, \gamma w_{b y}$ obey $C^{0}$ continuity and are represented by Lagrange interpolation functions while $w_{b}$ and $w_{s}$ obey $C^{1}$ continuity and are represented by Hermite functions.

$$
\begin{aligned}
\lfloor u\rfloor & =\left\lfloor H_{L}\right\rfloor\left\{q_{u}\right\} \\
\lfloor\gamma\rfloor & =\left\lfloor H_{L}\right\rfloor\left\{q_{\gamma}\right\} \\
\left\lfloor w_{b y}\right\rfloor & =\left\lfloor H_{L}\right\rfloor\left\{q_{w_{b}}\right\} \\
\left\lfloor w_{b}\right\rfloor & =\lfloor H\rfloor\left\{q_{w_{b}}\right\} \\
\left\lfloor w_{s}\right\rfloor & =\lfloor H\rfloor\left\{q_{w_{s}}\right\}
\end{aligned}
$$

The virtual forms of the above are

$$
\begin{gathered}
\lfloor\delta u\rfloor=\left\lfloor\delta q_{u}\right\rfloor\left\{H_{L}\right\} \\
\lfloor\delta \gamma\rfloor=\left\lfloor\delta q_{\gamma}\right\rfloor\left\{H_{L}\right\} \\
\left\lfloor\delta w_{b}\right\rfloor=\left\lfloor\delta q_{w_{b}}\right\rfloor\{H\} \\
\left\lfloor\delta w_{s}\right\rfloor=\left\lfloor\delta q_{w_{s}}\right\rfloor\{H\} \\
\left\lfloor\delta w_{b y}\right\rfloor=\left\lfloor\delta q_{w_{b}^{y}}\right\rfloor\left\{H_{L}\right\}
\end{gathered}
$$

Substituting the above eqs in Eq. (3.61) and Eq. (3.80), the virtual strain energy can be represented as

$$
\delta U=\left\lfloor\delta q_{e}\right\rfloor\left[K_{e}\right]\left\{q_{e}\right\}
$$

The stiffness matrix is composed of 49 sub matrices. The elastic sub matrices are represented as $\left[K_{u u}\right],\left[K_{\gamma \gamma}\right],\left[K_{w_{b} w_{b}}\right],\left[K_{w_{s} w_{s}}\right],\left[K_{w_{b}^{y} w_{b}^{y}}\right],\left[K_{u \gamma}\right],\left[K_{u w_{b}}\right],\left[K_{u w_{s}}\right],\left[K_{u w_{b y}}\right],\left[K_{\gamma w_{b}}\right],\left[K_{\gamma w_{s}}\right]$, $\left[K_{\gamma w_{b}^{y}}\right],\left[K_{w_{b} w_{s}}\right],\left[K_{w_{b} w_{b}^{y}}\right],\left[K_{w_{s} w_{b}^{y}}\right]$ with dimensions $5 \times 5,5 \times 5,7 \times 7,7 \times 7,5 \times 5,5 \times 5,5 \times 7,5 \times 7$, $5 \times 5,5 \times 7,5 \times 7,5 \times 5,7 \times 7,7 \times 5$ and $7 \times 5$ respectively. The piezoelectric sub matrices are are $\left[K_{u \varphi_{a}}\right]$, 
$\left[K_{\gamma \varphi_{a}}\right],\left[K_{w_{b} \varphi_{a}}\right],\left[K_{w_{s} \varphi_{a}}\right],\left[K_{w_{b}^{y} \varphi_{a}}\right],\left[K_{u \varphi_{s}}\right],\left[K_{\gamma \varphi_{s}}\right],\left[K_{w_{b} \varphi_{s}}\right],\left[K_{w_{s} \varphi_{s}}\right]$ and $\left[K_{w_{b}^{y} \varphi_{s}}\right]$. The electrical sub matrices are $\left[K_{\varphi_{a} \varphi_{a}}\right]$ and $\left[K_{\varphi_{s} \varphi_{s}}\right]$.

The sub matrices are given as

$$
\begin{aligned}
& {\left[K_{u u}\right]=b \int_{0}^{l_{e}} \bar{S}_{11}\left\{H_{L}^{\prime}\right\}\left[H_{L}^{\prime}\right\rfloor d x} \\
& {\left[K_{u \gamma}\right]=b \int_{0}^{l_{e}} \bar{S}_{12}\left\{H_{L}^{\prime}\right\}\left\lfloor H_{L}\right\rfloor d x} \\
& {\left[K_{u w_{b}}\right]=-b \int_{0}^{l_{e}} \bar{S}_{13}\left\{H_{L}^{\prime}\right\}\left\lfloor H^{\prime \prime}\right] d x} \\
& {\left[K_{u w_{s}}\right]=[0]} \\
& \left.\left[K_{u w_{b}^{y}}\right]=-2 b \int_{0}^{l_{e}} \bar{S}_{14}\left\{H_{L}^{\prime}\right\} \mid H_{L}^{\prime}\right] d x \\
& {\left[K_{\gamma \gamma}\right]=b \int_{0}^{l_{e}} \bar{S}_{22}\left\{H_{L}\right\}\left\lfloor H_{L}\right\rfloor d x} \\
& {\left[K_{\gamma w_{b}}\right]=b \int_{0}^{l_{e}} \bar{S}_{23}\left\{H_{L}\right\}\left[H^{\prime \prime}\right] d x} \\
& {\left[K_{\gamma w_{s}}\right]=[0]} \\
& {\left[K_{\gamma w_{b}^{y}}\right]=-2 b \int_{0}^{l_{e}} \bar{S}_{24}\left\{H_{L}\right\}\left[H_{L}^{\prime}\right] d x} \\
& \left.\left.\left[K_{w_{b} w_{b}}\right]=b \int_{0}^{l_{e}} \bar{S}_{33}\left\{H^{\prime \prime}\right\} \mid H^{\prime \prime}\right] d x+b \int_{0}^{l_{e}} N_{x}\left\{H^{\prime}\right\} \mid H^{\prime}\right] d x \\
& {\left[K_{w_{b} w_{s}}\right]=[0]} \\
& \left.\left[K_{w_{b} w_{b}^{y}}\right]=2 b \int_{0}^{l_{e}} \bar{S}_{34}\left\{H^{\prime \prime}\right\} \mid H_{L}^{\prime}\right] d x \\
& {\left[K_{w_{s} w_{s}}\right]=b \int_{0}^{l_{e}} A^{*}\left\{H^{\prime \prime}\right\}\left\lfloor H^{\prime \prime}\right\rfloor d x+b \int_{0}^{l_{e}} N_{x}\left\{H^{\prime}\right\}\left\lfloor H^{\prime}\right\rfloor d x}
\end{aligned}
$$




$$
\begin{aligned}
& {\left[K_{w_{b}^{y} w_{b}^{y}}\right]=4 b \int_{0}^{l_{e}} \bar{S}_{34}\left\{H_{L}^{\prime}\right\}\left\lfloor H_{L}^{\prime}\right\rfloor d x} \\
& {\left[K_{w_{s} w_{b y}}\right]=[0]} \\
& {\left[K_{u \varphi_{a}}\right]=b \int_{0}^{l_{e}} S_{P 1 a}\left\{H_{L}^{\prime}\right\} d x} \\
& {\left[K_{\gamma \varphi_{a}}\right]=b \int_{0}^{l_{e}} S_{P 2 a}\left\{H_{L}\right\} d x} \\
& {\left[K_{w_{b} \varphi_{a}}\right]=-b \int_{0}^{l_{e}} S_{P 3 a}\left\{H^{\prime \prime}\right\} d x} \\
& {\left[K_{w_{s} \varphi_{a}}\right]=[0]} \\
& {\left[K_{w_{b}^{y} \varphi_{a}}\right]=-2 b \int_{0}^{l_{e}} S_{P 4 a}\left\{H_{L}^{\prime}\right\} d x} \\
& {\left[K_{u \varphi_{s}}\right]=b \int_{0}^{l_{e}} S_{P 1 s}\left\{H_{L}^{\prime}\right\} d x} \\
& {\left[K_{\gamma \varphi_{s}}\right]=b \int_{0}^{l_{e}} S_{P 2 s}\left\{H_{L}\right\} d x} \\
& {\left[K_{w_{b} \varphi_{s}}\right]=-b \int_{0}^{l_{e}} S_{P 3 s}\left\{H^{\prime \prime}\right\} d x} \\
& {\left[K_{w_{s} \varphi_{a}}\right]=[0]} \\
& {\left[K_{w_{b}^{y}} \varphi_{s}\right]=-2 b \int_{0}^{l_{e}} S_{P 4 s}\left\{H_{L}^{\prime}\right\} d x} \\
& {\left[K_{\varphi_{a} \varphi_{a}}\right]=-\frac{b}{t_{k}} \int_{0}^{l_{e}}\left(\epsilon_{33}\right)_{a} d x-\frac{b}{t_{k}} \int_{0}^{l_{e}} D_{\varphi_{a}} d x} \\
& {\left[K_{\varphi_{s} \varphi_{s}}\right]=-\frac{b}{t_{k}} \int_{0}^{l_{e}}\left(\epsilon_{33}\right)_{s} d x-\frac{b}{t_{k}} \int_{0}^{l_{e}} D_{\varphi_{s}} d x}
\end{aligned}
$$

The element stiffness matrix is 


$$
\left[K_{e}\right]=\left[\begin{array}{ccccccc}
{\left[K_{u u}\right]} & {\left[K_{u \gamma}\right]} & {\left[K_{u w_{b}}\right]} & {\left[K_{u w_{s}}\right]} & {\left[K_{u w_{b}^{y}}\right]} & {\left[K_{u \varphi_{a}}\right]} & {\left[K_{u \varphi_{s}}\right]} \\
{\left[K_{u \gamma}\right]^{T}} & {\left[K_{\gamma \gamma}\right]} & {\left[K_{\gamma w_{b}}\right]} & {\left[K_{\gamma w_{s}}\right]} & {\left[K_{\gamma w_{b}^{y}}\right]} & {\left[K_{\gamma \varphi_{a}}\right]} & {\left[K_{\gamma \varphi_{s}}\right]} \\
{\left[K_{u w_{b}}\right]^{T}} & {\left[K_{\gamma w_{b}}\right]^{T}} & {\left[K_{w_{b} w_{b}}\right]} & {\left[K_{w_{b} w_{s}}\right]} & {\left[K_{w_{b} w_{b}^{y}}\right]} & {\left[K_{w_{b} \varphi_{a}}\right]} & {\left[K_{w_{b} \varphi_{s}}\right]} \\
{\left[K_{u w_{s}}\right]^{T}} & {\left[K_{\gamma w_{s}}\right]^{T}} & {\left[K_{w_{b} w_{s}}\right]^{T}} & {\left[K_{w_{s} w_{s}}\right]} & {\left[K_{w_{s} w_{b}^{y}}\right]} & {\left[K_{w_{s} \varphi_{a}}\right]} & {\left[K_{w_{s} \varphi_{s}}\right]} \\
{\left[K_{u w_{b}^{y}}\right]^{T}} & {\left[K_{\gamma w_{b}^{y}}\right]^{T}} & {\left[K_{w_{b} w_{b}^{y}}\right]^{T}} & {\left[K_{w_{s} w_{b}^{y}}\right]^{T}} & {\left[K_{w_{b}^{y} w_{b}^{y}}\right]} & {\left[K_{w_{b}^{y} \varphi_{a}}\right]} & {\left[K_{w_{b}^{y} \varphi_{s}}\right]} \\
{\left[K_{u \varphi_{a}}\right]^{T}} & {\left[K_{\gamma \varphi_{a}}\right]^{T}} & {\left[K_{w_{b} \varphi_{a}}\right]^{T}} & {\left[K_{w_{s} \varphi_{a}}\right]^{T}} & {\left[K_{w_{b}^{y} \varphi_{a}}\right]^{T}} & {\left[K_{\varphi_{a} \varphi_{a}}\right]} & {\left[K_{\varphi_{a} \varphi_{s}}\right]} \\
{\left[K_{u \varphi_{s}}\right]^{T}} & {\left[K_{\gamma \varphi_{s}}\right]^{T}} & {\left[K_{w_{b} \varphi_{s}}\right]^{T}} & {\left[K_{w_{s} \varphi_{s}}\right]^{T}} & {\left[K_{w_{b}^{y} \varphi_{s}}\right]^{T}} & {\left[K_{\varphi_{s} \varphi_{a}}\right]} & {\left[K_{\varphi_{s} \varphi_{s}}\right]}
\end{array}\right]
$$

The above equation can be written as

$$
\left[K_{e}\right]=\left[\begin{array}{cc}
{\left[K_{S S}\right]} & {\left[K_{s \varphi}\right]} \\
{\left[K_{S \varphi}\right]^{T}} & {\left[K_{\varphi \varphi}\right]}
\end{array}\right]
$$

where $\left[K_{s S}\right],\left[K_{s \varphi}\right]$ and $\left[K_{\varphi \varphi}\right]$ represent the elastic, piezoelectric and electric stiffness matrices.

\subsubsection{Element Stiffness matrix for reduced beam formulation using HSDT}

The beam element using HSDT has the following degrees of freedom

At end nodes for HSDT: $u, \gamma, w_{b}, w_{b}^{\prime}, w_{s}, w_{s}^{\prime}, w_{b}^{y}, w_{s}^{y}$

At mid side nodes for HSDT: $u, \gamma, w_{b}, w_{s}, w_{b}^{y}, w_{s}^{y}$

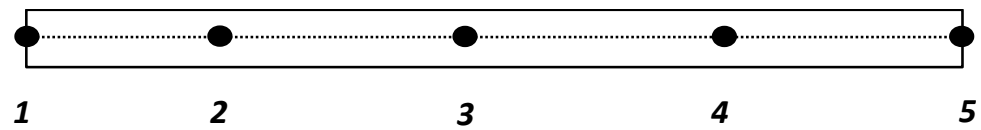

For nodes (1, 5): $u, \gamma, w_{b}, w_{b}^{\prime}, w_{s}, w_{s}^{\prime}, w_{b y}, w_{s y}$

For nodes $(2,3,4): u, \gamma, w_{b}, w_{s}, w_{b y}, w_{s y}$

Figure 4.5 Element degrees of freedom for beam formulation using HSDT 
The element has a total of 34 degrees of freedom; among which the variables $u, \gamma, w_{b y}$ and $w_{s y}$ assume $C^{0}$ continuity and are represented by Lagrange interpolation functions while $w_{b}$ and $w_{S}$ assume $C^{1}$ continuity and are represented by Hermite interpolation functions.

$$
\begin{gathered}
\lfloor u\rfloor=\left\lfloor H_{L}\right\rfloor\left\{q_{u}\right\} \\
\lfloor\gamma\rfloor=\left\lfloor H_{L}\right\rfloor\left\{q_{\gamma}\right\} \\
\left\lfloor w_{b}^{y}\right\rfloor=\left\lfloor H_{L}\right\rfloor\left\{q_{w_{b}^{y}}\right\} \\
\left\lfloor w_{b}\right\rfloor=\lfloor H\rfloor\left\{q_{w_{b}}\right\} \\
\left\lfloor w_{s}^{y}\right\rfloor=\left\lfloor H_{L}\right\rfloor\left\{q_{w_{s}^{y}}\right\} \\
\left\lfloor w_{s}\right\rfloor=\lfloor H\rfloor\left\{q_{w_{s}}\right\}
\end{gathered}
$$

And the virtual forms of the variables are,

$$
\begin{gathered}
\lfloor\delta u\rfloor=\left\lfloor\delta q_{u}\right\rfloor\left\{H_{L}\right\} \\
\lfloor\delta \gamma\rfloor=\left\lfloor\delta q_{\gamma}\right\rfloor\left\{H_{L}\right\} \\
\left\lfloor\delta w_{b}\right\rfloor=\left\lfloor\delta q_{w_{b}}\right\rfloor\{H\} \\
\left\lfloor\delta w_{s}\right\rfloor=\left\lfloor\delta q_{w_{s}}\right\rfloor\{H\} \\
\left\lfloor\delta w_{b}^{y}\right\rfloor=\left\lfloor\delta q_{w_{b}^{y}}\right\rfloor\left\{H_{L}\right\} \\
\left\lfloor\delta w_{s}^{y}\right\rfloor=\left\lfloor\delta q_{w_{s}^{y}}\right\rfloor\left\{H_{L}\right\}
\end{gathered}
$$

The stiffness matrix is obtained from the virtual mechanical strain energy equation Eq. (3.71) and the virtual electrical strain energy expression, Eq. (3.84).

$$
\delta U=\left\lfloor\delta q_{e}\right\rfloor\left[K_{e}\right]\left\{q_{e}\right\}
$$


The element stiffness matrix is partitioned into 64 sub matrices. The sub matrices for the elastic $\operatorname{part} \operatorname{are}\left[K_{u u}\right],\left[K_{\gamma \gamma}\right],\left[K_{w_{b} w_{b}}\right],\left[K_{w_{s} w_{s}}\right],\left[K_{w_{b}^{y} w_{b}^{y}}\right],\left[K_{w_{s}^{y} w_{s}^{y}}\right],\left[K_{u \gamma}\right],\left[K_{u w_{b}}\right],\left[K_{u w_{s}}\right],\left[K_{u w_{b}^{y}}\right]$, $\left[K_{u w_{s}^{y}}\right],\left[K_{\gamma w_{b}}\right],\left[K_{\gamma w_{s}}\right],\left[K_{\gamma w_{b y}}\right],\left[K_{\gamma w_{s}^{y}}\right],\left[K_{w_{b} w_{s}}\right],\left[K_{w_{b} w_{b}^{y}}\right],\left[K_{w_{b} w_{s}^{y}}\right],\left[K_{w_{s} w_{b}^{y}}\right],\left[K_{w_{s} w_{s}^{y}}\right]$ and $\left[K_{w_{b}^{y} w_{s}^{y}}\right]$ with dimensions $5 \times 5,5 \times 5,7 \times 7,7 \times 7,5 \times 5,5 \times 5,5 \times 5,5 \times 7,5 \times 7,5 \times 5,5 \times 5,5 \times 7$, $5 \times 7,5 \times 5,5 \times 5,7 \times 7,7 \times 5,7 \times 5,7 \times 5,7 \times 5,5 \times 5$ each. The sub matrices for the piezoelectric part $\operatorname{are}\left[K_{u \varphi_{a}}\right],\left[K_{\gamma \varphi_{a}}\right],\left[K_{w_{b} \varphi_{a}}\right],\left[K_{w_{s} \varphi_{a}}\right],\left[K_{w_{b}^{y} \varphi_{a}}\right],\left[K_{w_{s}^{y} \varphi_{a}}\right]$ and $\left[K_{u \varphi_{s}}\right],\left[K_{\gamma \varphi_{s}}\right],\left[K_{w_{b} \varphi_{s}}\right],\left[K_{w_{s} \varphi_{s}}\right]$ $\left[K_{w_{b}^{y} \varphi_{s}}\right],\left[K_{w_{s}^{y}} \varphi_{s}\right]$. The sub matrices for the electrical part are $\left[K_{\varphi_{a} \varphi_{a}}\right]$ and $\left[K_{\varphi_{S} \varphi_{s}}\right]$.

The assembled stiffness matrix is given by

$\left[K_{e}\right]=\left[\begin{array}{cccccccc}{\left[K_{u u}\right]} & {\left[K_{u \gamma}\right]} & {\left[K_{u w_{b}}\right]} & {\left[K_{u w_{s}}\right]} & {\left[K_{u w_{b}^{y}}\right]} & {\left[K_{u w_{s}^{y}}\right]} & {\left[K_{u \varphi_{a}}\right]} & {\left[K_{u \varphi_{s}}\right]} \\ {\left[K_{u \gamma}\right]^{T}} & {\left[K_{\gamma \gamma}\right]} & {\left[K_{\gamma w_{b}}\right]} & {\left[K_{\gamma w_{s}}\right]} & {\left[K_{\gamma w_{b}^{y}}\right]} & {\left[K_{\gamma w_{s}^{y}}\right]} & {\left[K_{\gamma \varphi_{a}}\right]} & {\left[K_{\gamma \varphi_{s}}\right]} \\ {\left[K_{u w_{b}}\right]^{T}} & {\left[K_{\gamma w_{b}}\right]^{T}} & {\left[K_{w_{b} w_{b}}\right]} & {\left[K_{w_{b} w_{s}}\right]} & {\left[K_{w_{b} w_{b}^{y}}\right]} & {\left[K_{w_{b} w_{s}^{y}}\right]} & {\left[K_{w_{b} \varphi_{a}}\right]} & {\left[K_{w_{b} \varphi_{s}}\right]} \\ {\left[K_{u w_{s}}\right]^{T}} & {\left[K_{\gamma w_{s}}\right]^{T}} & {\left[K_{w_{b} w_{s}}\right]^{T}} & {\left[K_{w_{s} w_{s}}\right]} & {\left[K_{w_{s} w_{b}^{y}}\right]} & {\left[K_{w_{s} w_{s}^{y}}\right]} & {\left[K_{w_{s} \varphi_{a}}\right]} & {\left[K_{w_{s} \varphi_{s}}\right]} \\ {\left[K_{u w_{b}^{y}}\right]^{T}} & {\left[K_{\gamma w_{b}^{y}}\right]^{T}} & {\left[K_{w_{b} w_{b}^{y}}\right]^{T}} & {\left[K_{w_{s} w_{b}^{y}}\right]^{T}} & {\left[K_{w_{b}^{y} w_{b}^{y}}\right]} & {\left[K_{w_{b}^{y} w_{s}^{y}}\right]} & {\left[K_{w_{b}^{y} \varphi_{a}}\right]} & {\left[K_{w_{b}^{y} \varphi_{s}}\right]} \\ {\left[K_{u w_{s}^{y}}\right]^{T}} & {\left[K_{\gamma w_{s}^{y}}\right]^{T}} & {\left[K_{w_{b} w_{s}^{y}}\right]^{T}} & {\left[K_{w_{s} w_{s}^{y}}\right]^{T}} & {\left[K_{w_{b}^{y} w_{s}^{y}}\right]^{T}} & {\left[K_{w_{s}^{y} w_{s}^{y}}\right]} & {\left[K_{w_{s}^{y} \varphi_{a}}\right]} & {\left[K_{w_{s}^{y} \varphi_{s}}\right]} \\ {\left[K_{u \varphi_{a}}\right]^{T}} & {\left[K_{\gamma \varphi_{a}}\right]^{T}} & {\left[K_{w_{b} \varphi_{a}}\right]^{T}} & {\left[K_{w_{s} \varphi_{a}}\right]^{T}} & {\left[K_{w_{b}^{y} \varphi_{a}}\right]^{T}} & {\left[K_{w_{s}^{y} \varphi_{a}}\right]^{T}} & {\left[K_{\varphi_{a} \varphi_{a}}\right]} & {\left[K_{\varphi_{a} \varphi_{s}}\right]} \\ {\left[K_{u \varphi_{s}}\right]^{T}} & {\left[K_{\gamma \varphi_{s}}\right]^{T}} & {\left[K_{w_{b} \varphi_{s}}\right]^{T}} & {\left[K_{w_{s} \varphi_{s}}\right]^{T}} & {\left[K_{w_{b}^{y} \varphi_{s}}\right]^{T}} & {\left[K_{w_{s}^{y} \varphi_{s}}\right]^{T}} & {\left[K_{\varphi_{s} \varphi_{a}}\right]} & {\left[K_{\varphi_{s} \varphi_{s}}\right]}\end{array}\right]$

The element stiffness matrix can be written as

$$
\left[K_{e}\right]=\left[\begin{array}{cc}
{\left[K_{S S}\right]} & {\left[K_{s \varphi}\right]} \\
{\left[K_{S \varphi}\right]^{T}} & {\left[K_{\varphi \varphi}\right]}
\end{array}\right]
$$

$\left[K_{S S}\right],\left[K_{s \varphi}\right]$ and $\left[K_{\varphi \varphi}\right]$ represent the elastic, piezoelectric and electric stiffness matrices. 


\subsection{Incremental stiffness matrix}

The incremental stiffness matrix is the time dependent part of the stiffness matrix. It enters the stiffness matrix formulation through the axial stress resultant term $N_{x}$ and is defined as $F_{x} / b$.

The axial force $F_{x}$ depends on the acceleration provided to the beam for axial motion and given by Eq. (3.5) as.

$$
a^{L}=-A \Omega^{2} \sin (\Omega t)
$$

The strain energy corresponding to the axial forces is given by

$$
\begin{gathered}
U_{F_{x}}=\frac{b}{2} \int_{0}^{l_{e}} N_{x} w^{\prime 2} d x \\
U_{F_{x}}=\frac{1}{2} \int_{0}^{l_{e}} F_{x} w^{\prime 2} d x
\end{gathered}
$$

The virtual strain energy due to axial forces is

$$
\delta U_{F_{x}}=\int_{0}^{l_{e}} F_{x} w^{\prime} \delta w^{\prime} d x
$$

Using Newton's second law, the axial force is expressed in terms of mass per unit length, $\gamma$ and acceleration, $a^{L}$ and thus

Eq. (4.32) becomes

$$
\delta U_{F_{x}}=-\int_{0}^{l_{e}} \gamma(L-x) a^{L} w^{\prime} \delta w^{\prime} d x
$$

Expressing $w^{\prime}$ in terms of Hermite interpolation functions, 


$$
\delta U_{F_{x}}=-\int_{0}^{l_{e}} \gamma(L-x) a^{L}\left\lfloor\delta q_{w}\right\rfloor\left\{H^{\prime}\right\}\left\lfloor H^{\prime}\right]\left\{q_{w}\right\} d x
$$

where $\left\{q_{w}\right\}$ and $\left[\delta q_{w}\right\rfloor$ are the transverse displacements and its variations.

The incremental stiffness matrix is obtained as

$$
\left[K_{F_{x}}\right]=-a^{L} \int_{0}^{l_{e}} \gamma(L-x)\left\{H^{\prime}\right\}\left\lfloor H^{\prime}\right\rfloor d x
$$

The above equation in non- dimensional form is written as

$$
\left[K_{F_{x}}\right]=-\frac{2 a^{L}}{l_{e}} \int_{-1}^{1} \gamma\left\{L-\left[x_{b}+\frac{l_{e}}{2}(1+\xi)\right]\right\}\left\{H^{\prime}(\xi)\right\}\left[H^{\prime}(\xi)\right] d \xi
$$

Equation (4.36) is added to the $\left[K_{w w}\right]$ term of the formulation using CLPT. In the formulation using FSDT and HSDT it enters the stiffness matrix through $\left[K_{w_{b} w_{b}}\right]$ and $\left[K_{w_{s} w_{s}}\right]$.

\subsection{Element mass matrix formulation}

Element Inertia matrix is obtained from virtual kinetic expression as

$$
-\delta T_{e}=\left\lfloor\delta q_{e}\right\rfloor\left[M_{e}\right]\left\{q_{e}\right\}
$$

where $\delta T_{e}$ is the element virtual kinetic energy and $\left[M_{e}\right]$ represents the element inertia matrix which depends on the composite plate theory under consideration.

\subsubsection{Element Inertia matrix using CLPT}

The inertia matrix for beam using CLPT is obtained substituting the Lagrangian interpolation functions for $u, \gamma$ and $w^{y}$ and Hermite interpolation functions for $w$ in Eq. (3.92). The obtained inertia matrix is composed of sub matrices and represented as 


$$
\left[M_{e}\right]=\left[\begin{array}{cccc}
{\left[m_{u u}\right]} & {\left[m_{u \gamma}\right]} & {\left[m_{u w}\right]} & {\left[m_{u w^{y}}\right]} \\
{\left[m_{u \gamma}\right]^{T}} & {\left[m_{\gamma \gamma}\right]} & {\left[m_{\gamma w}\right]} & {\left[m_{\gamma w^{y}}\right]} \\
{\left[m_{u w}\right]^{T}} & {\left[m_{\gamma w}\right]^{T}} & {\left[m_{w w}\right]} & {\left[m_{w w^{y}}\right]} \\
{\left[m_{u w^{y}}\right]^{T}} & {\left[m_{\gamma w^{y}}\right]^{T}} & {\left[m_{w w^{y}}\right]^{T}} & {\left[m_{w^{y} w^{y}}\right]}
\end{array}\right]
$$

The sub matrices are given by

$$
\begin{gathered}
{\left[M_{u u}\right]=b \int_{0}^{l_{e}} I_{0}\left\{H_{L}\right\}\left\lfloor H_{L}\right\rfloor d x_{e}} \\
{\left[M_{u w}\right]=-b \int_{0}^{l_{e}} I_{1}\left\{H_{L}\right\}\left[H^{\prime}\right] d x_{e}} \\
{\left[M_{w u}\right]=\left[M_{u w}\right]^{T}} \\
{\left[M_{w w}\right]=b \int_{0}^{l_{e}}\left(I_{0}\{H\}[H\rfloor+I_{2}\left\{H^{\prime}\right\}\left[H^{\prime}\right]\right) d x_{e}} \\
{\left[M_{w^{y}} w^{y}\right]=b \int_{0}^{l_{e}} I_{2}\left\{H_{L}\right\}\left[H_{L}\right] d x_{e}} \\
{\left[M_{u \gamma}\right]=\left[M_{\gamma \gamma}\right]=\left[M_{\gamma w}\right]=\left[M_{\gamma w^{y}}\right]=\left[M_{w w^{y}}\right]=\left[M_{u w^{y}}\right]=0}
\end{gathered}
$$

\subsubsection{Element Inertia matrix for beam using FSDT}

The element inertia matrix beam using FSDT is obtained substituting the Lagrangian interpolation functions for $u, \gamma$ and $w_{b}^{y}$ and Hermite interpolation functions for $w_{b}$ and $w_{s}$ in Eq. (3.100).

The obtained inertia matrix is composed of sub matrices and represented as 


$$
M_{e}=\left[\begin{array}{ccccc}
{\left[M_{u u}\right]} & {\left[M_{u \gamma}\right]} & {\left[M_{u w_{b}}\right]} & {\left[M_{u w_{s}}\right]} & {\left[M_{u w_{b}^{y}}\right]} \\
{\left[M_{u \gamma}\right]^{T}} & {\left[M_{\gamma \gamma}\right]} & {\left[M_{\gamma w_{b}}\right]} & {\left[M_{\gamma w_{s}}\right]} & {\left[M_{\gamma w_{b}^{y}}\right]} \\
{\left[M_{u w_{b}}\right]^{T}} & {\left[M_{\gamma w_{b}}\right]^{T}} & {\left[M_{w_{b} w_{b}}\right]} & {\left[M_{w_{b} w_{s}}\right]} & {\left[M_{w_{b} w_{b}^{y}}\right]} \\
{\left[M_{u w_{s}}\right]^{T}} & {\left[M_{\gamma w_{s}}\right]^{T}} & {\left[M_{w_{b} w_{s}}\right]^{T}} & {\left[M_{w_{s} w_{s}}\right]} & {\left[M_{w_{s} w_{b}^{y}}\right]} \\
{\left[M_{u w_{b}^{y}}\right]^{T}} & {\left[M_{\gamma w_{b}^{y}}\right]^{T}} & {\left[M_{w_{b} w_{b}^{y}}\right]^{T}} & {\left[M_{w_{s} w_{b}^{y}}\right]^{T}} & {\left[M_{w_{b} w_{b}^{y}}\right]}
\end{array}\right]
$$

The sub matrices are given by

$$
\begin{aligned}
& {\left[M_{u u}\right]=b \int_{0}^{l_{e}} I_{0}\left\{H_{L}\right\}\left\lfloor H_{L}\right\rfloor d x_{e}} \\
& \left.\left[M_{u w_{b}}\right]=-b \int_{0}^{l_{e}} I_{1}\left\{H_{L}\right\} \mid H^{\prime}\right] d x_{e} \\
& {\left[M_{w u}\right]=\left[M_{u w}\right]^{T}} \\
& {\left[M_{w_{b} w_{b}}\right]=b \int_{0}^{l_{e}}\left(I_{0}\{H\}\lfloor H\rfloor+I_{2}\left\{H^{\prime}\right\}\left[H^{\prime}\right]\right) d x_{e}} \\
& {\left[M_{w_{b} w_{s}}\right]=b \int_{0}^{l_{e}} I_{0}\{H\}\lfloor H\rfloor d x_{e}} \\
& {\left[M_{w_{s} w_{s}}\right]=b \int_{0}^{l_{e}} I_{0}\{H\}\lfloor H\rfloor d x_{e}} \\
& {\left[M_{w_{b} w_{b}}\right]=b \int_{0}^{l_{e}} I_{2}\left\{H_{L}\right\}\left[H_{L}\right] d x_{e}} \\
& {\left[M_{u \gamma}\right]=\left[M_{u w_{s}}\right]=\left[M_{u w_{b y}}\right]=\left[M_{\gamma \gamma}\right]=\left[M_{\gamma w_{b}}\right]=\left[M_{\gamma w_{b}^{y}}\right]=0} \\
& {\left[M_{\gamma w_{s}}\right]=\left[M_{w_{b} w_{b}^{y}}\right]=\left[M_{w_{s} w_{b}^{y}}\right]=0}
\end{aligned}
$$




\subsubsection{Element Inertia matrix for beam using HSDT}

The element inertia matrix for beam using HSDT is obtained substituting the Lagrangian interpolation functions for $u, \gamma, w_{b}^{y}$ and $w_{s}^{y}$ and Hermite interpolation functions for $w_{b}$ and $w_{s}$ in Eq. (3.105).

The obtained inertia matrix is composed of sub matrices and represented as

$$
\left[M_{e}\right]=\left[\begin{array}{cccccc}
{\left[M_{u u}\right]} & {\left[M_{u \gamma}\right]} & {\left[M_{u w_{b}}\right]} & {\left[M_{u w_{s}}\right]} & {\left[M_{u w_{b}^{y}}\right]} & {\left[M_{u w_{s}^{y}}\right]} \\
{\left[M_{u \gamma}\right]^{T}} & {\left[M_{\gamma \gamma}\right]} & {\left[M_{\gamma w_{b}}\right]} & {\left[M_{\gamma w_{s}}\right]} & {\left[M_{\gamma w_{b}^{y}}\right]} & {\left[M_{\gamma w_{s}^{y}}\right]} \\
{\left[M_{u w_{b}}\right]^{T}} & {\left[M_{\gamma w_{b}}\right]^{T}} & {\left[M_{w_{b} w_{b}}\right]} & {\left[M_{w_{b} w_{s}}\right]} & {\left[M_{w_{b} w_{b}^{y}}\right]} & {\left[M_{w_{b} w_{s}^{y}}\right]} \\
{\left[M_{u w_{s}}\right]^{T}} & {\left[M_{\gamma w_{s}}\right]^{T}} & {\left[M_{w_{b} w_{s}}\right]^{T}} & {\left[M_{w_{s} w_{s}}\right]} & {\left[M_{w_{s} w_{b}^{y}}\right]} & {\left[M_{w_{s} w_{s}^{y}}\right]} \\
{\left[M_{u w_{b}^{y}}\right]^{T}} & {\left[M_{\gamma w_{b}^{y}}\right]^{T}} & {\left[M_{w_{b} w_{b}^{y}}\right]^{T}} & {\left[M_{w_{s} w_{b}^{y}}\right]^{T}} & {\left[M_{w_{b}^{y} w_{b}^{y}}\right]} & {\left[M_{w_{b}^{y} w_{s}^{y}}\right]} \\
{\left[M_{u w_{s}^{y}}\right]^{T}} & {\left[M_{\gamma w_{s}^{y}}\right]^{T}} & {\left[M_{w_{b} w_{s}^{y}}\right]^{T}} & {\left[M_{w_{s} w_{s}^{y}}\right]^{T}} & {\left[M_{w_{s} w_{s}}\right]^{T}} & {\left[M_{w_{s} w_{s}^{y}}\right]}
\end{array}\right]
$$

The sub matrices are given by

$$
\begin{gathered}
{\left[M_{u u}\right]=b \int_{0}^{l_{e}} I_{0}\left\{H_{L}\right\}\left\lfloor H_{L}\right\rfloor d x_{e}} \\
{\left[M_{u w_{b}}\right]=-b \int_{0}^{l_{e}} I_{1}\left\{H_{L}\right\}\left\lfloor H^{\prime}\right\rfloor d x_{e}} \\
{\left[M_{u w_{s}}\right]=-b c_{1} \int_{0}^{l_{e}} I_{3}\left\{H_{L}\right\}\left\lfloor H^{\prime}\right\rfloor d x_{e}} \\
{\left[M_{w_{b} w_{b}}\right]=b \int_{0}^{l_{e}} I_{2}\left\{H^{\prime}\right\}\left\lfloor H^{\prime}\right\rfloor d x_{e}+b \int_{0}^{l_{e}} I_{0}\{H\}\lfloor H\rfloor d x_{e}} \\
{\left[M_{w_{b} w_{s}}\right]=b \int_{0}^{l_{e}} c_{1} I_{4}\left\{H^{\prime}\right\}\left\lfloor H^{\prime}\right\rfloor d x_{e}+b \int_{0}^{l_{e}} I_{0}\{H\}\lfloor H\rfloor d x_{e}}
\end{gathered}
$$




$$
\begin{aligned}
& {\left[M_{w_{s} w_{s}}\right]=b \int_{0}^{l_{e}} c_{1}^{2} I_{6}\left\{H^{\prime}\right\}\left\lfloor H^{\prime}\right\rfloor d x_{e}+b \int_{0}^{l_{e}} I_{0}\{H\}\lfloor H\rfloor d x_{e}} \\
& {\left[M_{w_{b} w_{b}^{y}}\right]=b \int_{0}^{l_{e}} I_{2}\left\{H_{L}\right\}\left\lfloor H_{L}\right\rfloor d x_{e}} \\
& {\left[M_{w_{b} w_{s}^{y}}\right]=b c_{1} \int_{0}^{l_{e}} I_{4}\left\{H_{L}\right\}\left\lfloor H_{L}\right\rfloor d x_{e}} \\
& {\left[M_{w_{s}} w_{s}^{y}\right]=b c_{1}^{2} \int_{0}^{l_{e}} I_{6}\left\{H_{L}\right\}\left\lfloor H_{L}\right\rfloor d x_{e}} \\
& {\left[M_{u \gamma}\right]=\left[M_{u w_{b}^{y}}\right]=\left[M_{u w_{s}^{y}}\right]=\left[M_{\gamma w_{b}}\right]=\left[M_{\gamma w_{s}}\right]=0} \\
& {\left[M_{\gamma w_{b}^{y}}\right]=\left[M_{\gamma w_{s}^{y}}\right]=\left[M_{w_{b} w_{b}^{y}}\right]=\left[M_{w_{b} w_{s}^{y}}\right]=\left[M_{w_{s} w_{b}^{y}}\right]=0}
\end{aligned}
$$

\subsection{Equation of motion}

The equation of motion is obtained as

$$
\left[\begin{array}{cc}
{\left[M_{s S}\right]} & 0 \\
0 & 0
\end{array}\right]\left\{\begin{array}{c}
\left\{\ddot{q_{s}}\right\} \\
\{\ddot{\varphi}\}
\end{array}\right\}+\left[\begin{array}{cc}
{\left[K_{s S}\right]} & {\left[K_{s \varphi}\right]} \\
{\left[K_{s \varphi}\right]^{T}} & {\left[K_{\varphi \varphi}\right]}
\end{array}\right]\left\{\begin{array}{c}
\left\{q_{s}\right\} \\
\{\varphi\}
\end{array}\right\}=\left\{\begin{array}{c}
\{Q\} \\
-\left\{F_{q}\right\}
\end{array}\right\}
$$

Where $\{Q\}$ and $\left\{F_{q}\right\}$ denote the structural force and the force due to electric charge, respectively.

Expanding Eq. (4.44),

$$
\begin{gathered}
{\left[M_{S S}\right]\left\{\ddot{q}_{s}\right\}+\left[K_{S S}\right]\left\{q_{s}\right\}+\left[K_{s \varphi}\right]\{\varphi\}=\{Q\}} \\
{\left[K_{s \varphi}\right]^{T}\left\{q_{s}\right\}+\left[K_{\varphi \varphi}\right]\{\varphi\}=-\left\{F_{q}\right\}} \\
\{\varphi\}=-\left[K_{\varphi \varphi}\right]^{-1}\left\{F_{q}\right\}-\left[K_{\varphi \varphi}\right]^{-1}\left[K_{s \varphi}\right]^{T}\left\{q_{s}\right\}
\end{gathered}
$$

i.e., 
Thus, Eq. (4.45) becomes:

$$
\left[M_{s S}\right]\left\{\ddot{q}_{s}\right\}+\left(\left[K_{S S}\right]-\left[K_{s \varphi}\right]\left[K_{\varphi \varphi}\right]^{-1}\left[K_{s \varphi}\right]^{T}\right)\left\{q_{s}\right\}=\{Q\}+\left[K_{s \varphi}\right]\left[K_{\varphi \varphi}\right]^{-1}\left\{F_{q}\right\}
$$

For the sensor layer the applied charge is 0 and thus $\left\{F_{q}\right\}$ is $\{0\}$.

The voltage developed at the sensor layer is obtained from Eq. (4.47)

$$
\left\{\varphi_{s}\right\}=-\left[K_{\varphi \varphi}\right]_{S}^{-1}\left[K_{s \varphi}\right]_{S}^{T}\left\{q_{s}\right\}
$$

The voltage supplied to the actuator is obtained by employing negative gain velocity feedback control as

$$
\left\{\varphi_{a}\right\}=-G\left\{\dot{\varphi}_{s}\right\}
$$

Writing Eq. (4.48) in terms actuator voltage,

$$
\left[M_{s S}\right]\left\{\ddot{q}_{s}\right\}+\left(\left[K_{s S}\right]-\left[K_{s \varphi}\right]\left[K_{\varphi \varphi}\right]^{-1}\left[K_{s \varphi}\right]^{\prime}\right)\left\{q_{s}\right\}=\{Q\}+\left[K_{s \varphi}\right] \varphi_{a}
$$

Therefore the equation of motion is

$$
\left[M_{e}\right]\left\{\ddot{q}_{s}\right\}+\left[C_{e}\right]\left\{\dot{q}_{s}\right\}+\left[\widehat{K}_{e}\right]\left\{q_{s}\right\}=0
$$

Where

$$
\begin{gathered}
{\left[C_{e}\right]=-G\left[K_{S \varphi}\right]\left[K_{\varphi \varphi}\right]_{S}^{-1}\left[K_{S \varphi}\right]_{S}^{T}} \\
{\left[\widehat{K}_{e}\right]=\left[K_{S S}\right]-\left[K_{S \varphi}\right]\left[K_{\varphi \varphi}\right]^{-1}\left[K_{s \varphi}\right]^{T}} \\
{\left[M_{e}\right]=\left[M_{S S}\right]}
\end{gathered}
$$

The element mass, damping and the stiffness matrices are assembled to form Global mass, damping and stiffness matrices respectively.

Therefore the global equation of motion is

$$
[M]\left\{\ddot{q}_{s}\right\}+[C]\left\{\dot{q}_{s}\right\}+[K]\left\{q_{s}\right\}=0
$$




\subsection{Lagrange Multipliers}

In the present work, constraints at the support locations of the moving beam are applied via Lagrange multiplier approach. In the case of moving beams, the support's location changes with time and doesn't fall at the nodes every time. In such cases, Lagrange multiplier approach is suitable to apply the constraints.

The total potential energy of the beam is given by

$$
\Delta_{P}=\delta U-\delta T-\delta W
$$

Where $\delta U, \delta T, \delta W$ are the virtual strain energy, virtual kinetic energy and virtual external work done on the beam. On introducing the Lagrange multipliers, the above equation is modified to

$$
\bar{\Delta}_{P}=(\delta U-\delta T-\delta W)+\delta(\ldots)
$$

The terms in the second parenthesis depends on the composite plate theory applied.

For reduced beam formulation using CLPT, the constraints are applied on $w$ and $w_{y}$

$$
\bar{\Delta}_{P}=(\delta U-\delta T-\delta W)+\delta\left(\left.\lambda_{1} w\right|_{x_{s 1}}+\left.\lambda_{2} w_{y}\right|_{x_{s 1}}+\left.\lambda_{3} w\right|_{x_{s 2}}+\left.\lambda_{4} w_{y}\right|_{x_{s 2}}\right)
$$

For reduced beam formulation using FSDT, the constraints are applied on $w_{b}, w_{s}, w_{b}^{y}$

$$
\begin{aligned}
\bar{\Delta}_{P}=(\delta U-\delta T & -\delta W) \\
& +\delta\left(\left.\lambda_{1} w_{b}\right|_{x_{s 1}}+\left.\lambda_{2} w_{s}\right|_{x_{s 1}}+\left.\lambda_{3} w_{b}^{y}\right|_{x_{s 1}}+\left.\lambda_{4} w_{b}\right|_{x_{s 2}}+\left.\lambda_{5} w_{s}\right|_{x_{s 2}}\right. \\
& \left.+\left.\lambda_{6} w_{b}^{y}\right|_{x_{s 2}}+\left.\lambda_{7} w_{s}^{y}\right|_{x_{s 2}}\right)
\end{aligned}
$$

For reduced beam formulation using HSDT, the constraints are applied on $w_{b}, w_{s}, w_{b}^{y}, w_{s}^{y}$

$$
\begin{aligned}
\bar{\Delta}_{P}=(\delta U-\delta T & -\delta W) \\
& +\delta\left(\left.\lambda_{1} w_{b}\right|_{x_{s 1}}+\left.\lambda_{2} w_{s}\right|_{x_{s 1}}+\left.\lambda_{3} w_{b}^{y}\right|_{x_{s 1}}+\left.\lambda_{4} w_{s}^{y}\right|_{x_{s 1}}+\left.\lambda_{5} w_{b}\right|_{x_{s 2}}\right. \\
& \left.+\left.\lambda_{6} w_{s}\right|_{x_{s 2}}+\left.\lambda_{7} w_{b}^{y}\right|_{x_{s 2}}+\left.\lambda_{8} w_{s}^{y}\right|_{x_{s 2}}\right)
\end{aligned}
$$


where $\lambda_{i}$ are the Lagrange multipliers corresponding to the constraints at the two supports. For a non-moving beam, Lagrange multipliers are applied to inertia, damping and stiffness matrices to constrain acceleration, velocity and displacement at the supports.

The modified stiffness, inertia and damping matrices are given by

$$
\begin{aligned}
{[K] } & =\left[\begin{array}{cc}
{[K]} & {\left[K_{\lambda}\right]} \\
{\left[K_{\lambda}^{T}\right]} & 0
\end{array}\right] \\
{[M] } & =\left[\begin{array}{cc}
{[M]} & {\left[M_{\lambda}\right]} \\
{\left[M_{\lambda}^{T}\right]} & 0
\end{array}\right] \\
{[C] } & =\left[\begin{array}{cc}
{[C]} & {\left[C_{\lambda}\right]} \\
{\left[C_{\lambda}^{T}\right]} & 0
\end{array}\right]
\end{aligned}
$$

where $\left[K_{\lambda}\right],\left[M_{\lambda}\right]$ and $\left[C_{\lambda}\right]$ are the Lagrange multiplier matrices. Lagrange multiplier matrices for stiffness, mass and inertia matrix are same but represented with different symbols.

For a moving beam, only displacements are constrained at the supports and Lagrange multipliers are applied to the stiffness matrix. Velocities and accelerations are not constrained.

The modified stiffness, inertia and damping matrices are

$$
\begin{gathered}
{[K]=\left[\begin{array}{cc}
{[K]} & {\left[K_{\lambda}\right]} \\
{\left[K_{\lambda}^{T}\right]} & {[0]}
\end{array}\right]} \\
{[M]=\left[\begin{array}{ll}
{[M]} & {[0]} \\
{[0]} & {[0]}
\end{array}\right]} \\
{[C]=\left[\begin{array}{ll}
{[C]} & {[0]} \\
{[0]} & {[0]}
\end{array}\right]}
\end{gathered}
$$

The Lagrange multipliers method works well if the number of constraints is much less than the total degrees of freedom of the structure. The Lagrange multiplier method avoids the additional step of determining the reaction forces. For a simply supported beam subjected to a uniformly distributed load, Lagrange multiplier values give the reactions at the supports. 


\section{NUMERICAL METHODS}

Numerical techniques used in this research are presented in this chapter. Gauss quadrature is used to perform intergration of elemental stiffness, inertia and damping matrices. Newmark's implicit time integration method is used to find out the time dependent damped response of the structure. Boundary and initial conditions for beams formulated using different plate theories is presented here.

\subsection{Gauss quadrature}

The Gauss quadrature is used for the numerical integration of element stiffness, inertia and damping matrices and load vectors in the spatial coordinate. It has been proven [19], [20] that the gauss quadrature is more effective for integration when applying finite element method. For exact integration of a polynomial of order (2n-1), Gauss quadrature requires $n$ eually spaced sampling points. The integration scheme can be represented as

$$
\int_{-1}^{1} f(x) d x=\sum_{j=1}^{n} w_{j} f\left(a_{j}\right)
$$

Table 5.1shows the sampling points and their corresponding weights used in the present work.

Table 5.1 Gauss quadrature sampling points and weights

\begin{tabular}{|l|l|}
\hline \multicolumn{1}{|c|}{ Sampling points } & Weights \\
\hline \pm 0.9491079123 & 0.1294849661 \\
\hline \pm 0.7415311855 & 0.0797053914 \\
\hline \pm 0.4058451513 & 0.3813005050 \\
\hline 0.0000000000 & 0.4179591836 \\
\hline
\end{tabular}




\subsection{Time integration scheme}

In general, the response in the time domain is evaluated using two common methods, namely direct integration and mode superposition techniques.

In Direct integration methods, additional transformation of equations using the eigenvectors is not required. It is based on the following assumptions:

a. The equation of equilibrium is satisfied at discrete time intervals, $\Delta t$

b. The displacements, velocities, and accelerations vary within each interval.

In the present study, direct integration methods are considered with focus on the Newmark method. This is an extension of the linear acceleration method. This method is unconditionally stable and more accurate [19] than other direct integration methods. It is an implicit integration scheme as the equilibrium is considered at $t+\Delta t$. Therefore the equilibrium equation is written as

$$
[M]\left\{\ddot{q}_{t+\Delta t}\right\}+[C]\left\{\dot{q}_{t+\Delta t}\right\}+[K]\left\{q_{t+\Delta t}\right\}=\{Q\}
$$

Where $[M],[C]$, and $[K]$ are the global inertia, damping, and stiffness matrices and $\{Q\}$ is the global load vector.

The displacements and velocities at time $t+\Delta t$ are assumed as

$$
\begin{gathered}
\left\{\dot{q}_{t+\Delta t}\right\}=\left\{\dot{q}_{t}\right\}+\left[(1-\delta)\left\{\ddot{q}_{t}\right\}+\delta\left\{\ddot{q}_{t+\Delta t}\right\}\right] \Delta t \\
\left\{q_{t+\Delta t}\right\}=\left\{q_{t}\right\}+\left\{\dot{q}_{t}\right\} \Delta t+\left[\left(\frac{1}{2}-\alpha\right)\left\{\ddot{q}_{t}\right\}+\alpha\left\{\ddot{q}_{t+\Delta t}\right\}\right] \Delta t^{2}
\end{gathered}
$$


where $\alpha$ and $\delta$ are Newmark parameters. Setting $\alpha=\frac{1}{4}$ and $\delta=\frac{1}{2}$ reduces the Newmark method to the constant acceleration method which is unconditionally stable and accurate.

\subsubsection{Step by step procedure for the Newmark method}

\section{Initial Calculations}

1. Form the global stiffness matrix $[K]$, inertia matrix $[M]$ and damping matrix $[C]$.

2. Initialize $U_{0}, \dot{U}_{0}$ and $\ddot{U}_{0}$.

3. Select time step size $\Delta t$, parameters $\alpha$ and $\delta$, and calculate the integration constants.

$$
\begin{aligned}
& \delta \geq 0.50 ; \text { and } \alpha \geq 0.25(0.5+\delta)^{2} \\
& a_{0}=\frac{1}{\alpha \Delta t^{2}} ; \quad a_{1}=\frac{\delta}{\alpha \Delta t} ; \quad a_{2}=\frac{1}{\alpha \Delta t} ; \quad a_{3}=\frac{1}{2 \alpha}-1 \\
& a_{4}=\frac{\delta}{\alpha}-1 ; \quad a_{5}=\frac{\Delta t}{2}\left(\frac{\delta}{\alpha}-2\right) ; \quad a_{6}=\Delta t(1-\delta) ; \quad a_{7}=\delta \Delta t
\end{aligned}
$$

\section{For each time step}

1. Add the time dependent part of stiffness matrix.

2. Form effective stiffness matrix $[\widehat{K}]=[K]+a_{0}[M]+a_{1}[C]$

3. Triangularize $\widehat{\mathrm{K}}$.

4. Calculate the effective loads at time $t+\Delta t$

$$
\left\{\hat{Q}_{t+\Delta t}\right\}=\left\{Q_{t+\Delta t}\right\}+[M]\left(a_{0}\left\{q_{t}\right\}+a_{2}\{\dot{q}\}_{t}+a_{3}\left\{\ddot{q}_{t}\right\}\right)+[C]\left(a_{1}\left\{q_{t}\right\}+a_{4}\left\{\dot{q}_{t}\right\}+a_{5}\left\{\ddot{q}_{t}\right\}\right)
$$

5. Calculate the displacements at $\mathrm{t}+\Delta \mathrm{t}$

6. Calculate the velocities and accelerations at time $\mathrm{t}+\Delta \mathrm{t}$.

$$
\begin{gathered}
\left\{\ddot{q}_{t+\Delta t}\right\}=a_{0}\left(\left\{q_{t+\Delta t}\right\}-\left\{q_{t}\right\}\right)-a_{2}\left\{\dot{q}_{t}\right\}-a_{3}\left\{\ddot{q}_{t}\right\} \\
\left\{\dot{q}_{t+\Delta t}\right\}=\left\{\dot{q}_{t}\right\}+a_{6}\left\{\ddot{q}_{t}\right\}+a_{7}\left\{\ddot{q}_{t+\Delta t}\right\}
\end{gathered}
$$




\subsection{Boundary and initial conditions}

\subsubsection{Boundary conditions}

The boundary conditions considered in this research are given below

\subsubsection{Formulation based on CLPT}

In the formulation using CLPT, $u, \gamma, w$, and $w^{y}$ are the degrees of freedom at each node with $w$ having slope continuity at the end nodes. The boundary conditions for the two common support conditions are

Fixed support : $u=w=w^{\prime}=w^{y}=0$

Hinged support: $u=w=w^{y}=0$

\subsubsection{Formulation based on FSDT}

In the formulation using FSDT, $u, \gamma, w_{b}, w_{s}, w_{b}^{y}$ are the degrees of freedom at each nodewith $w_{b}, w_{s}$ having slope continuity at the end nodes. The boundary conditions for two common supports are

Fixed support: $u=w_{b}=w_{b}^{\prime}=w_{b}^{y}=w_{s}=w_{s}^{\prime}=0$

Hinged support: $u=w_{b}=w_{b}^{y}=w_{s}=0$

\subsubsection{Formulaltion based on HSDT}

In the formulation using HSDT, $u, \gamma, w_{b}, w_{s}, w_{b}^{y}, w_{s}^{y}$ are the degrees of freedom at each node with $w_{b}, w_{s}$ havin slope continuity at the end nodes. The boundary conditions at different supports are

Fixed support: $u=w_{b}=w_{b}^{\prime}=w_{b}^{y}=w_{s}=w_{s}^{\prime}=w_{s}^{y}=0$

Hinged support: $u=w_{b}=w_{b}^{y}=w_{s}=w_{s}^{y}=0$ 


\subsubsection{Initial conditions}

The $1^{\text {st }}$ mode shape in bending $(w)$ of the overhung beam placed symmetrically over the supports with tip deflection normalized to $0.005 \mathrm{~m}$ at the left and the right tips is taken as the initial condition for the formulation using CLPT, FSDT and HSDT. 


\section{RESULTS AND DISCUSSIONS}

In this chapter the response of a composite moving beam equipped with piezoelectric sensor and actuator layers is presented. Several verification examples are judiciously chosen. The results for the moving beam are presented in terms of tip deflections for CLPT, FSDT and HSDT.

\subsection{Verification of Composite moving beam}

The time dependet analysis of an initially deformed composite overhanging beam (Figuure 4.1) oscillating axially relative to the fixed supports is performed using the formulation proposed in the previous chapters. The finite element formulation is verified for all three plate theories. The geometric and material properties of the beam are taken from Nagappan (2000).

The geometric properties of the overhang beam under consideration are as follows:

Beam length $(L)=1.0 \mathrm{~m}$

Beam height $(h)=0.06 \mathrm{~m}$

Beam width $(b)=0.05 \mathrm{~m}$

Distance between the supports $(d)=0.25 L$

Initial distance of the left end of the beam from the first support, $S_{1},\left(X_{0}\right)=0.375 \mathrm{~L}$

Amplitude of axial rigid body motion $(A)=0.05 \mathrm{~m}$

Lay-ups considered: $[0 / \pm 45]$

The ply properties of the graphite-epoxy composite are:
$E_{1}=144.8 \mathrm{GPa}$
$G_{23}=3.448 \mathrm{GPa}$
$E_{2}=9.653 \mathrm{GPa}$
$\rho=1389.227 \mathrm{Kg} / \mathrm{m}^{3}$
$G_{12}=G_{13}=4.137 \mathrm{GPa}$
$v_{12}=0.3$ 
Overhanging beam is discritized into four elements with three internal nodes per each element. The beam given an initial deformation at time $t=0$ corresponding to the first bending mode shape of the beam normalized to have tip deflection of $0.005 \mathrm{~m}$ at the left and right end of the beam respectively. The beam is given an axial osciallation of $20 \mathrm{rad} / \mathrm{s}$. Newmark's time integration technique with a time step size of $2.5 \mathrm{E}-6$ is used to find out the time dependent response of the beam.

The left tip deflections of the overhanging moving beam using CLPT, FSDT and HSDT formulations is shown Figures 6.1-6.3. 


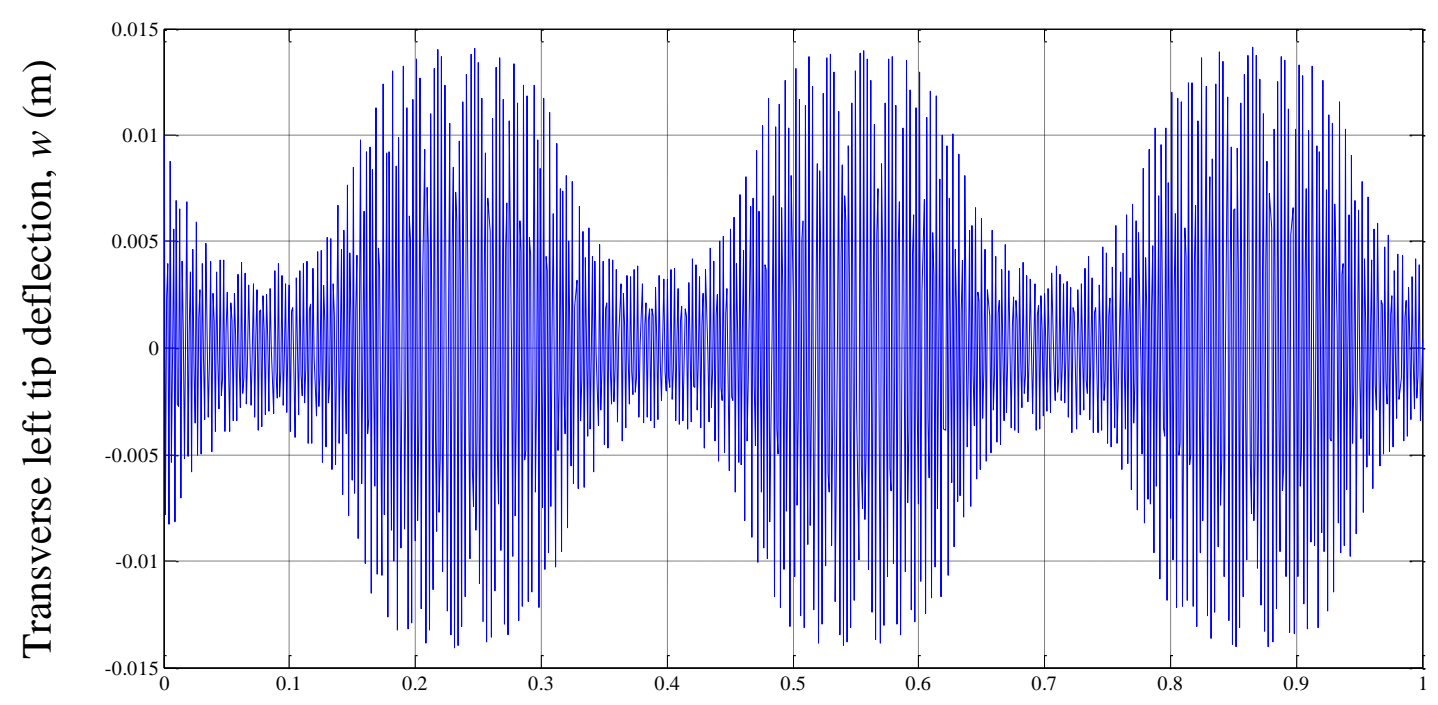

Time (s)

Figure 6.1 Transverse tip deflection at the left end, $w$, CLPT, $\Omega=20 \mathrm{rad} / \mathrm{s},[0 / \pm 45]_{\mathrm{s}}$ 


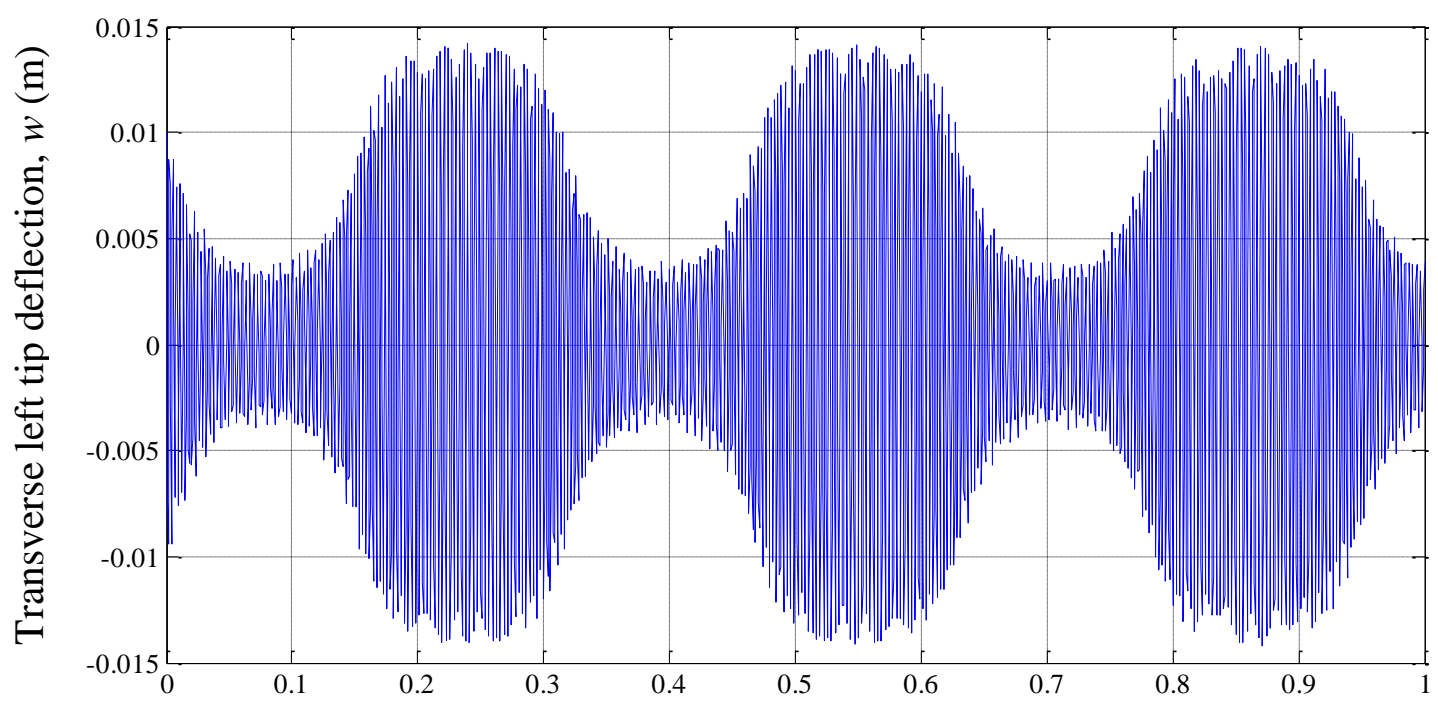

Time (s)

Figure 6.2 Transverse tip deflection at the left end, $w$, FSDT, $\Omega=20 \mathrm{rad} / \mathrm{s},[0 / \pm 45]_{\mathrm{s}}$ 


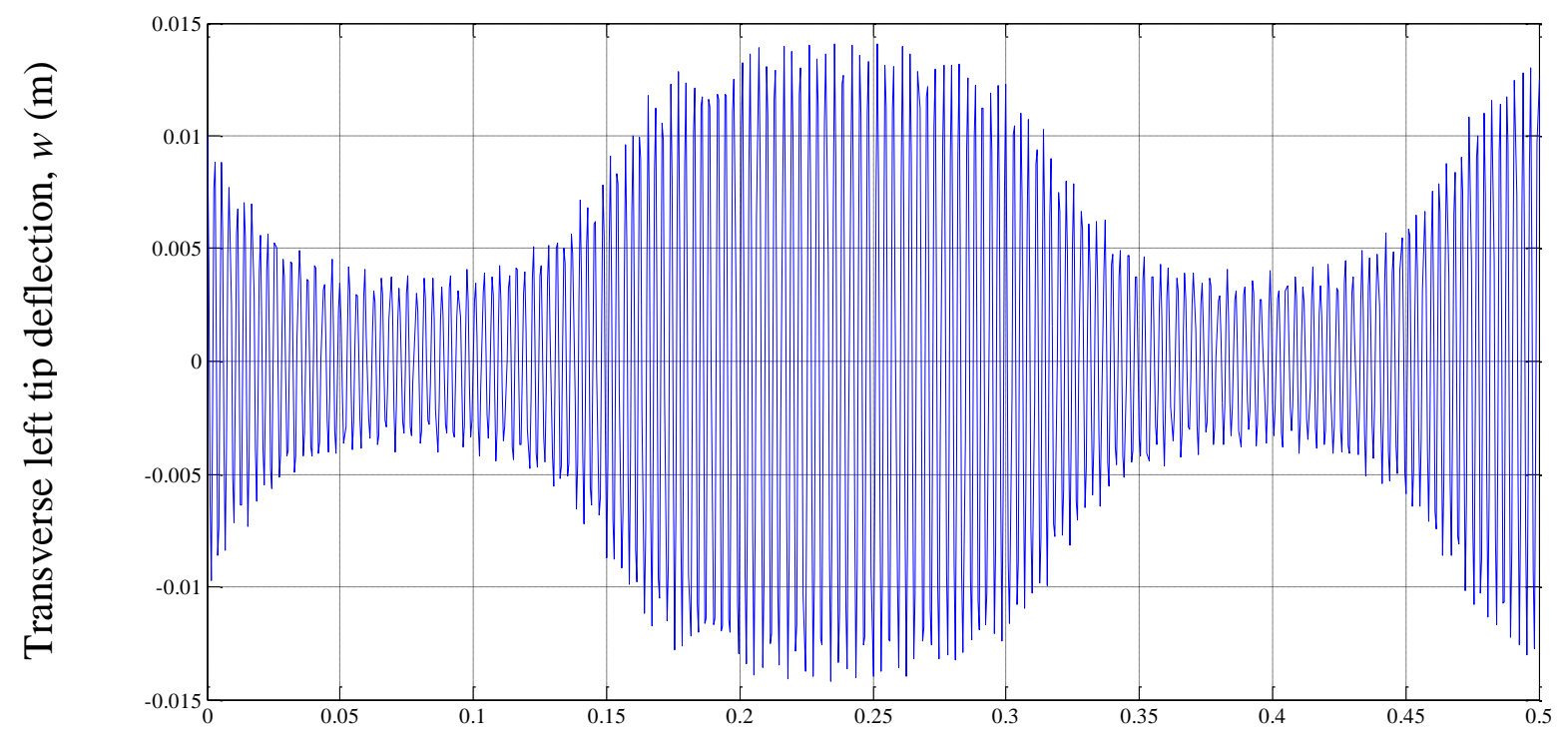

Time (s)

Figure 6.3 Transverse tip deflection at the left end, $w$, HSDT, $\Omega=20 \mathrm{rad} / \mathrm{s},[0 / \pm 45]_{\mathrm{s}}$ 


\subsection{Verification of piezoelectric formulation for a non moving beam}

The piezoelectric composite beam formultaion developed in the previous chapters is verfied by modeling a cantilever beam considered by Balamurugan and Narayanan (2002) as shown in Figure 6.4. Note that the top piezoelectric layer serves as the sensor while the bottom one as the actuator. An impulse load of $0.2 \mathrm{~N}$ is applied at the free end of the cantilever beam for $1 \mathrm{~ms}$. Piezoelectric control is applied after $0.5 \mathrm{~s}$ to distinguish between damped and undamped responses. A constant-voltage negative-velocity feedback control is used to communicate between sensors and actuators. Newmark's time integration technique is used to find the time dependent response of the model. A time step size of 2.5E-6 is used for the Newmark's method.

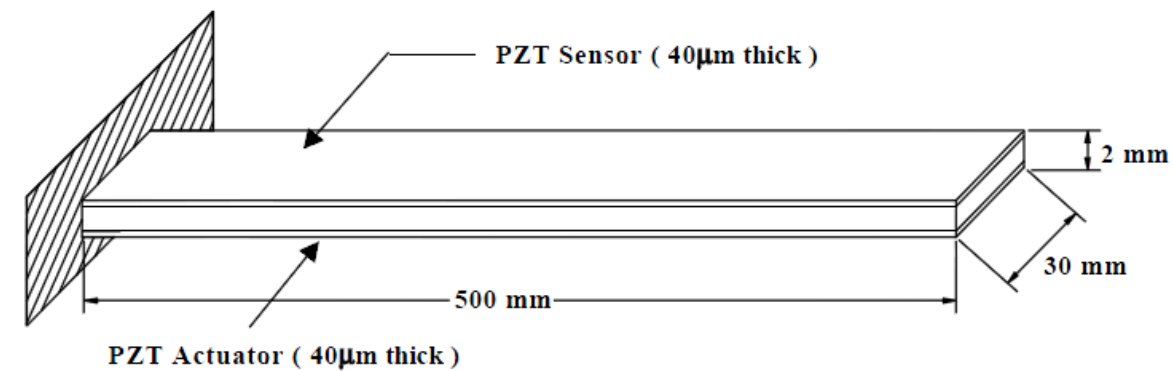

Figure 6.4 Cantilever beam with piezoelectric sensors and actuators [Balamurugan and Narayanan (2002)]

The material properties used are shown in Table 6.1

Table 6.1 Material properties [Balamurugan and Narayanan (2002)]

\begin{tabular}{|c|c|c|}
\hline Property name & Steel & PZT \\
\hline Young's modulus (-GPa-) & 210 & 139 \\
\hline Poisson's ratio & 0.3 & 0.3 \\
\hline Density $\left(-\mathrm{kg} / \mathrm{m}^{3}-\right)$ & 7850 & 7500 \\
\hline
\end{tabular}


The time dependent response of the tip of the cantilever beam is presented in Figure 6.1. The solid line represents the present results while the discrete points are from the reference and it is observed that they match very closely. 


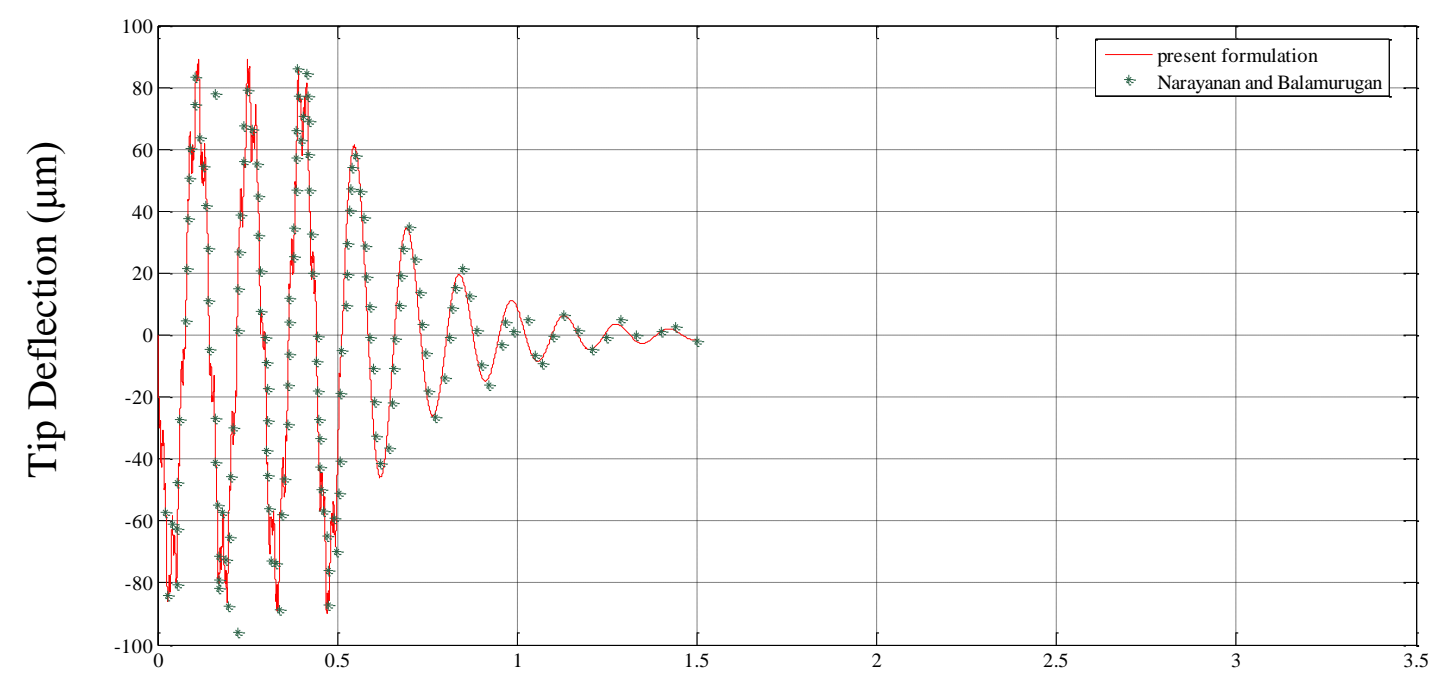

Time (s)

Figure 6.5 Tip deflection of a cantilever beam with piezoelectric layers on the top and bottom 


\subsection{Composite moving beam}

Controlled time response of a laminated composite moving beam attached with piezoelectric sensors and actuators is presented in this section. Results are produced for the moving beam formulated using different plate theories; CLPT, FSDT and HSDT. A parametric study is conducted for two different axial frequencies and different laminate lay ups. The material properties of the composite and the piezoelectric materials used in this research are presented in Table 6.2 and the geometric properties in Table 6.3. Note that the inherent material damping of the composite material is not taken into account in the present research.

Table 6.2 Material properties for the composite moving beam

\begin{tabular}{|c|c|c|}
\hline Property & $\begin{array}{c}\text { Carbon- epoxy [Barbero } \\
(2010)]\end{array}$ & PZT [Ha et al (1992)] \\
\hline$E_{1}(\mathrm{GPa})$ & 142 & 63 \\
\hline$E_{2}(\mathrm{GPa})$ & 10.3 & 63 \\
\hline$G_{12}(\mathrm{GPa})$ & 7.2 & 24.2 \\
\hline$G_{13}(\mathrm{GPa})$ & 7.2 & 24.2 \\
\hline$G_{23}(\mathrm{GPa})$ & 6 & 20 \\
\hline$v_{12}$ & 0.27 & 0.3 \\
\hline$d_{31}(\mathrm{~m} / \mathrm{V})$ & - & $2.54 \mathrm{E}-10$ \\
\hline$d_{32}(\mathrm{~m} / \mathrm{V})$ & - & $2.54 \mathrm{E}-10$ \\
\hline$\epsilon_{33}(\mathrm{~F} / \mathrm{m})$ & - & $1.53 \mathrm{E}-8$ \\
\hline
\end{tabular}

Table 6.3 Geometric properties of the composite moving beam

\begin{tabular}{|l|c|}
\hline \multicolumn{1}{|c|}{ Property } & Value \\
\hline Length, $L(\mathrm{~m})$ & 1 \\
\hline Width, $b(\mathrm{~m})$ & 0.05 \\
\hline Total thickness of carbon epoxy layers, $(\mathrm{m})$ & 0.06 \\
\hline Thickness of piezoelectric layer $(\mathrm{m})$ & $0.1 \mathrm{E}-3$ \\
\hline Distance between the supports, $\underline{d}(\mathrm{~m})$ & 0.25 \\
\hline Initial distance from the left end of the support $\mathrm{S}_{1}, X_{0}$ & $0.375 L$ \\
\hline Amplitude of axial rigid body motion, $A(\mathrm{~m})$ & 0.05 \\
\hline Frequency of axial motion, $\Omega(\mathrm{rad} / \mathrm{sec})$ & 10,20 \\
\hline Lay ups considered & {$[0 / \pm 20],[0 / \pm 45]$} \\
& {$[0 / \pm 45]_{\mathrm{s}}$} \\
\hline
\end{tabular}




\subsubsection{Moving beam simulation using CLPT}

Piezo electric sensors and actuators layers are affixed onto the top and bottom of the laminate. The beam given an initial deformation at time $t=0$ corresponding to the first bending mode shape of the beam normalized to have tip deflection of $0.005 \mathrm{~m}$ at the left and right end of the beam respectively. The beam is subjected to an axial motion

$$
X_{A}(t)=-X_{0}+A \sin (\Omega t)
$$

The piezoelectric sensor and actuator layers communicate with each other through a constant-gain negative-velocity feed back control, which means the gain is kept constant through out the response period. This combination provides an active damping effect to control the vibrations. The gains are selected such that the vibration amplitude of the left end of the beam reduces to the initial delfection by the end of 1 second.

The time response of the beam is obtained using Newmark's time integration scheme. A parametric study is conducted to select the time step size such that numerical divergence is avoided. A time step size of 2.5E-6 s is selected. The results are plotted over a time interval of 1 s. To have a distinction between the damped and undamped resonse, the damping is applied from $0.4 \mathrm{~s}$. The transvrse tip deflectionright ends of $[0 / \pm 45]$ beam with an axial oscillation frequency of $10 \mathrm{rad} / \mathrm{s}$ is shown in Figures 6.6 and 6.7.The response of beam shows a beat-like phenomenon, similar to that observed by Sivaneri and Nagappan (2012). The axial diaplacement $(u)$ at the left

end of the beam is fixed.. The axial diaplacement at the right end is shown in Figure 6.8. This also shows a beat-like phenomenon but at a lower level. It can be seen in all the cases that the amplitude of the peak goes down with the inclusion of damping. The effect of damping can be 
varied by varying the gains, depending on the requirement. Table 6.6 presents the transverse deflection of the left end of the beam.

For the same beam with axial frequency of $20 \mathrm{rad} / \mathrm{s}$, the transverse tip deflections and the axial displacements are shown in Figures 6.9 and 6.10, respectively. Similar beat-like phenomenon is seen but with a smaller beat period due to increased axial frequency. Table 6.7 presents the tip deflection at the left end of the beam. The gain applied to this beam to have the specified damping is higher than the previous case.

The response of the beam with $[0 / \pm 20]$ lay up with an axial frequency of $10 \mathrm{rad} / \mathrm{s}$ and 20 $\mathrm{rad} / \mathrm{s}$ is presented next. Similar beat-like phenomenon is seen with higher frequeny of response due to increased stiffness of the beam. The tip deflections at the right and left end of the beam are shown in Figure 6.12, figure 6.13, Figure 6.15 and Figure 6.16. The axial deflection at the right and left end of the beam is shown in Figure 6.14and Figure 6.17. Table 6.8 and Table 6.9 present the tip deflections at the left end of the beam for [0/ \pm 20$]$ lay up with axial frequencies 10 $\mathrm{rad} / \mathrm{s}$ and $20 \mathrm{rad} / \mathrm{s}$. The stiffness of this beam is much higher than the [0/ \pm 45$]$ beam and the axial deflection is much lower. Higher gains are required to suppress the amplitude.

The response of a symmetric beam with $[0 / \pm 45]_{\mathrm{s}}$ lay up with an axial frequency of 20 $\mathrm{rad} / \mathrm{s}$ is shown next. The transverse tip deflections for the right and the left end of the beam are shown in Figure 6.18 and Figure 6.19. Table 6.10 presents the tip deflection at the left end of the beam. The axial deflection at the right end is shown in Figure 6.20 and the amplitude is near zero until damping is introduced, as expected for a symmetric laminate. Axial deflections due to the inertial forces generated by the rigid-body axial motion are negligible for symmetric and unsymmetric laminates. 
The gains required for suppressing the additional amplitutde due to the axial motion of the beam for all the cases considered is presented in Table 6.4 and the magnification factors for the tip deflections due to axial oscillation for all the different laminate lay ups is summarized in Table 6.5.

Table 6.4 Feedback gains supplied to the beams

\begin{tabular}{|c|c|c|}
\hline Lay up & $\begin{array}{c}\text { Axial frequency, } \Omega \\
(\mathrm{rad} / \mathrm{s})\end{array}$ & Gain, $\mathrm{G}$ \\
\hline$[0 / \pm 20]$ & 10 & 0.22 \\
\hline$[0 / \pm 20]$ & 20 & 0.38 \\
\hline$[0 / \pm 45]$ & 10 & 0.018 \\
\hline$[0 / \pm 45]$ & 20 & 0.033 \\
\hline$[0 / \pm 45]_{\mathrm{s}}$ & 20 & 0.09 \\
\hline
\end{tabular}

Table 6.5 Magnification factors for the moving beams with CLPT formulation

\begin{tabular}{|c|c|c|c|c|}
\hline Lay up & frequency & $w_{\max }^{\text {static }}$ & $w_{\max }^{\text {moving }}$ & $\begin{array}{c}\text { Mag. factor } \\
=\frac{w_{\text {max }}^{\text {max }}}{w_{\text {maxi }}}\end{array}$ \\
\hline$[0 / \pm 20]$ & 10 & 0.005 & 0.006922 & 1.3844 \\
\hline$[0 / \pm 20]$ & 20 & 0.005 & 0.0070343 & 1.4069 \\
\hline$[0 / \pm 45]$ & 10 & 0.005 & 0.006858 & 1.3716 \\
\hline$[0 / \pm 45]$ & 20 & 0.005 & 0.0069703 & 1.3941 \\
\hline$[0 / \pm 45]_{\mathrm{s}}$ & 20 & 0.005 & 0.0070245 & 1.4049 \\
\hline
\end{tabular}




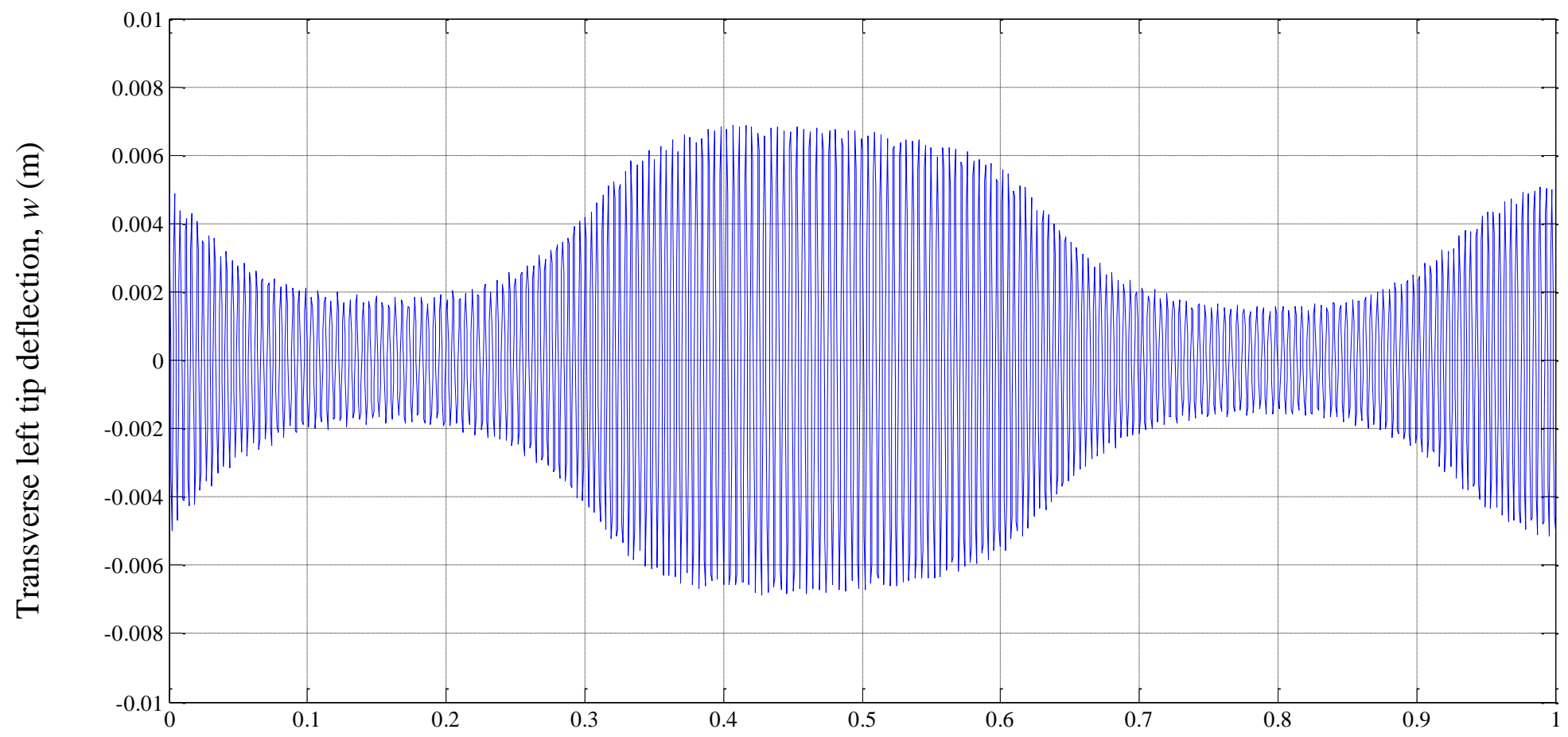

Time (s)

Figure 6.6 Transverse tip deflection at the left end, $w$, CLPT, $\Omega=10 \mathrm{rad} / \mathrm{s},[0 / \pm 45]$ 


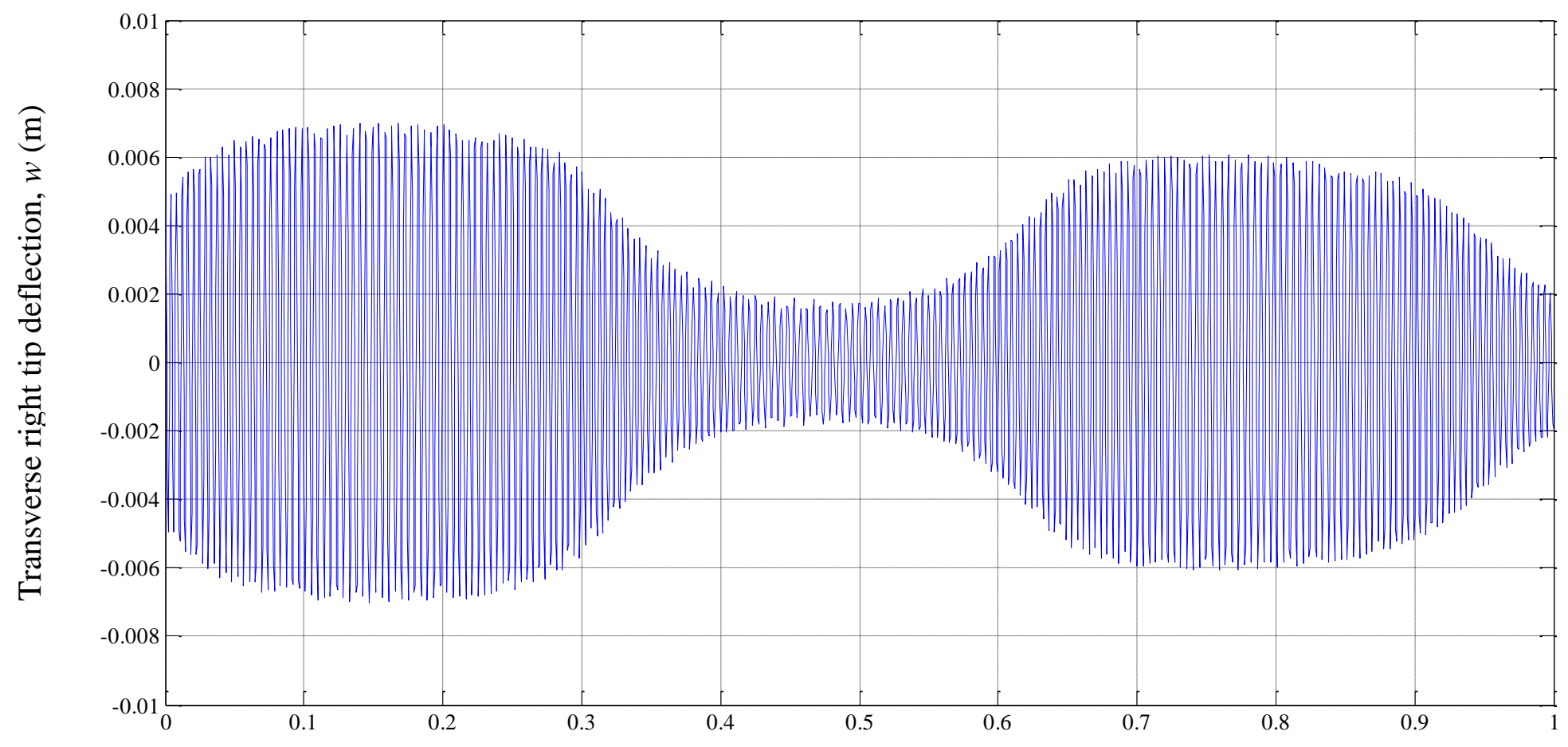

Time (s)

Figure 6.7 Transverse tip deflection at the right end, $w$, CLPT, $\Omega=10 \mathrm{rad} / \mathrm{s},[0 / \pm 45]$ 


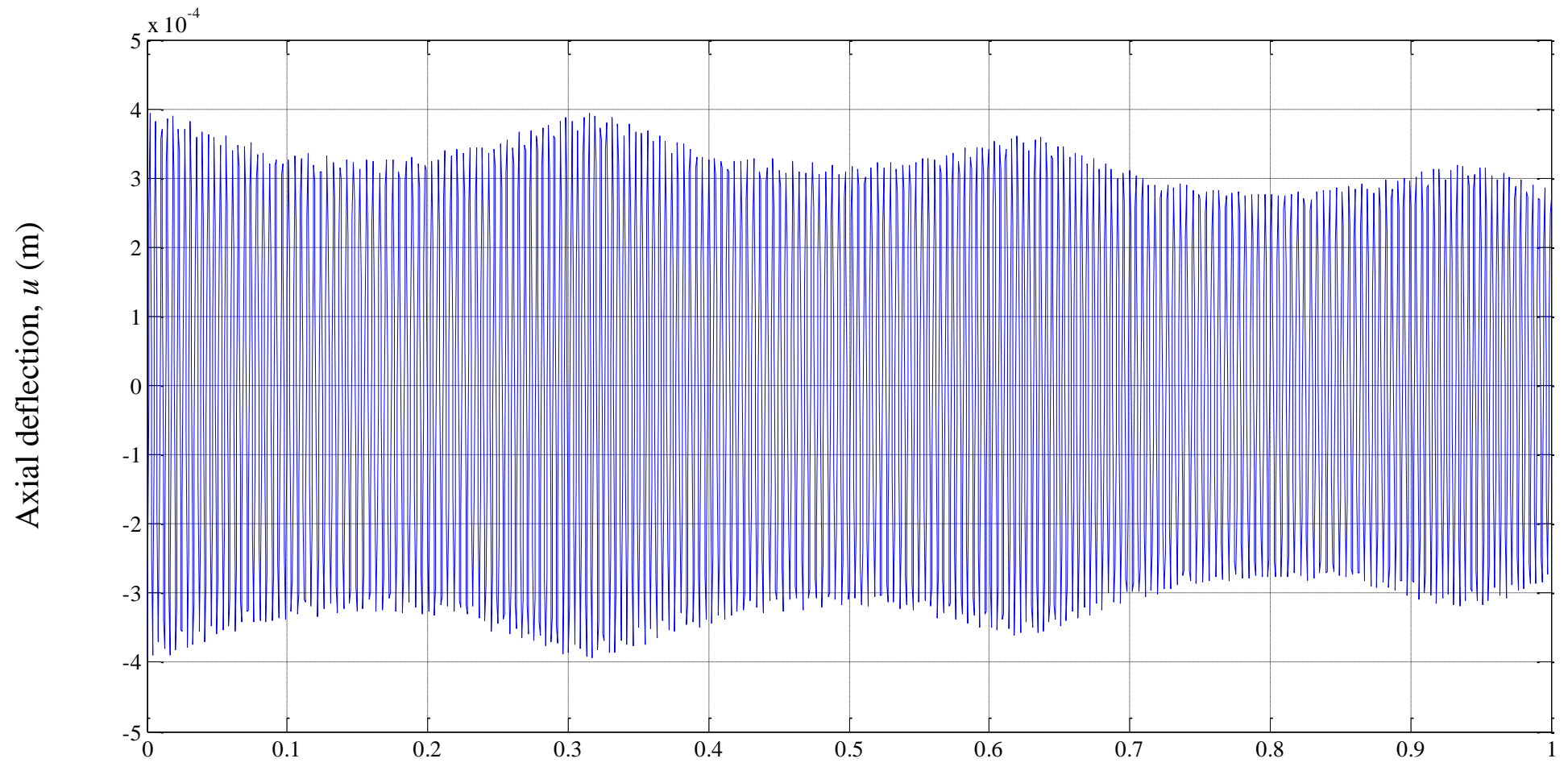

Time (s)

Figure 6.8 Axial deflection at the right end, $u$, CLPT, $\Omega=10 \mathrm{rad} / \mathrm{s},[0 / \pm 45]$ 
Table 6.6 Transverse tip deflection at the left end, $w$, CLPT, $\Omega=10 \mathrm{rad} / \mathrm{s},[0 / \pm 45]$

\begin{tabular}{|c|c|c|c|c|c|c|r|}
\hline$t(\mathrm{~s})$ & $w(\mathrm{~m})$ & $t(\mathrm{~s})$ & $w(\mathrm{~m})$ & $t(\mathrm{~s})$ & $w(\mathrm{~m})$ & $t(\mathrm{~s})$ & \multicolumn{1}{|c|}{$w(\mathrm{~m})$} \\
\hline 0.000000 & 0.005000 & 0.245500 & 0.002581 & 0.495000 & 0.006730 & 0.740500 & -0.001676 \\
\hline 0.016500 & 0.004282 & 0.256500 & -0.002786 & 0.502000 & -0.006717 & 0.756500 & 0.001643 \\
\hline 0.020500 & 0.004058 & 0.267000 & 0.003076 & 0.513500 & 0.006602 & 0.763500 & -0.001632 \\
\hline 0.031000 & -0.003679 & 0.277500 & -0.003267 & 0.525000 & -0.006619 & 0.770500 & 0.001617 \\
\hline 0.041500 & 0.003193 & 0.288000 & 0.003588 & 0.532000 & 0.006467 & 0.784500 & 0.001583 \\
\hline 0.054500 & 0.002826 & 0.298500 & -0.004114 & 0.541000 & 0.006444 & 0.791500 & -0.001567 \\
\hline 0.063000 & 0.002608 & 0.309000 & 0.004602 & 0.552000 & -0.006374 & 0.803500 & 0.001578 \\
\hline 0.074000 & -0.002485 & 0.319000 & -0.005206 & 0.563000 & 0.006221 & 0.819500 & -0.001606 \\
\hline 0.083000 & -0.002273 & 0.329500 & 0.005526 & 0.576000 & 0.006100 & 0.826500 & 0.001650 \\
\hline 0.098500 & 0.002120 & 0.335500 & -0.005839 & 0.582500 & -0.005940 & 0.833500 & -0.001682 \\
\hline 0.107500 & 0.002042 & 0.346000 & 0.006157 & 0.591000 & -0.005839 & 0.847000 & -0.001794 \\
\hline 0.114500 & -0.002037 & 0.356500 & -0.006281 & 0.601500 & 0.005585 & 0.856000 & -0.001906 \\
\hline 0.121500 & 0.001995 & 0.369500 & -0.006543 & 0.616000 & -0.005129 & 0.865000 & -0.002009 \\
\hline 0.135500 & 0.001915 & 0.371500 & 0.006591 & 0.620000 & -0.004927 & 0.876000 & 0.002084 \\
\hline 0.142500 & -0.001891 & 0.389000 & 0.006751 & 0.630500 & 0.004389 & 0.882500 & -0.002262 \\
\hline 0.156500 & -0.001861 & 0.398000 & 0.006839 & 0.640500 & -0.003899 & 0.897500 & 0.002498 \\
\hline 0.163500 & 0.001849 & 0.407000 & 0.006856 & 0.651000 & 0.003450 & 0.908000 & -0.002839 \\
\hline 0.170500 & -0.001839 & 0.416000 & 0.006859 & 0.661500 & -0.003094 & 0.918500 & 0.003228 \\
\hline 0.189000 & -0.001891 & 0.427500 & -0.006873 & 0.672000 & 0.002854 & 0.929000 & -0.003468 \\
\hline 0.198000 & -0.001928 & 0.439000 & 0.006838 & 0.680500 & 0.002580 & 0.935000 & 0.003808 \\
\hline 0.205000 & 0.002049 & 0.446000 & -0.006834 & 0.693500 & 0.002325 & 0.949500 & -0.004314 \\
\hline 0.212000 & -0.002130 & 0.453000 & 0.006824 & 0.700000 & -0.002143 & 0.953500 & -0.004357 \\
\hline 0.228000 & 0.002213 & 0.460000 & -0.006815 & 0.711000 & 0.002059 & 0.968000 & 0.004726 \\
\hline 0.236500 & 0.002325 & 0.474000 & -0.006789 & 0.720000 & 0.001942 & 0.978500 & -0.004966 \\
\hline 0.000000 & 0.005000 & 0.481000 & 0.006768 & 0.736000 & -0.001750 & 0.987000 & -0.005105 \\
\hline
\end{tabular}




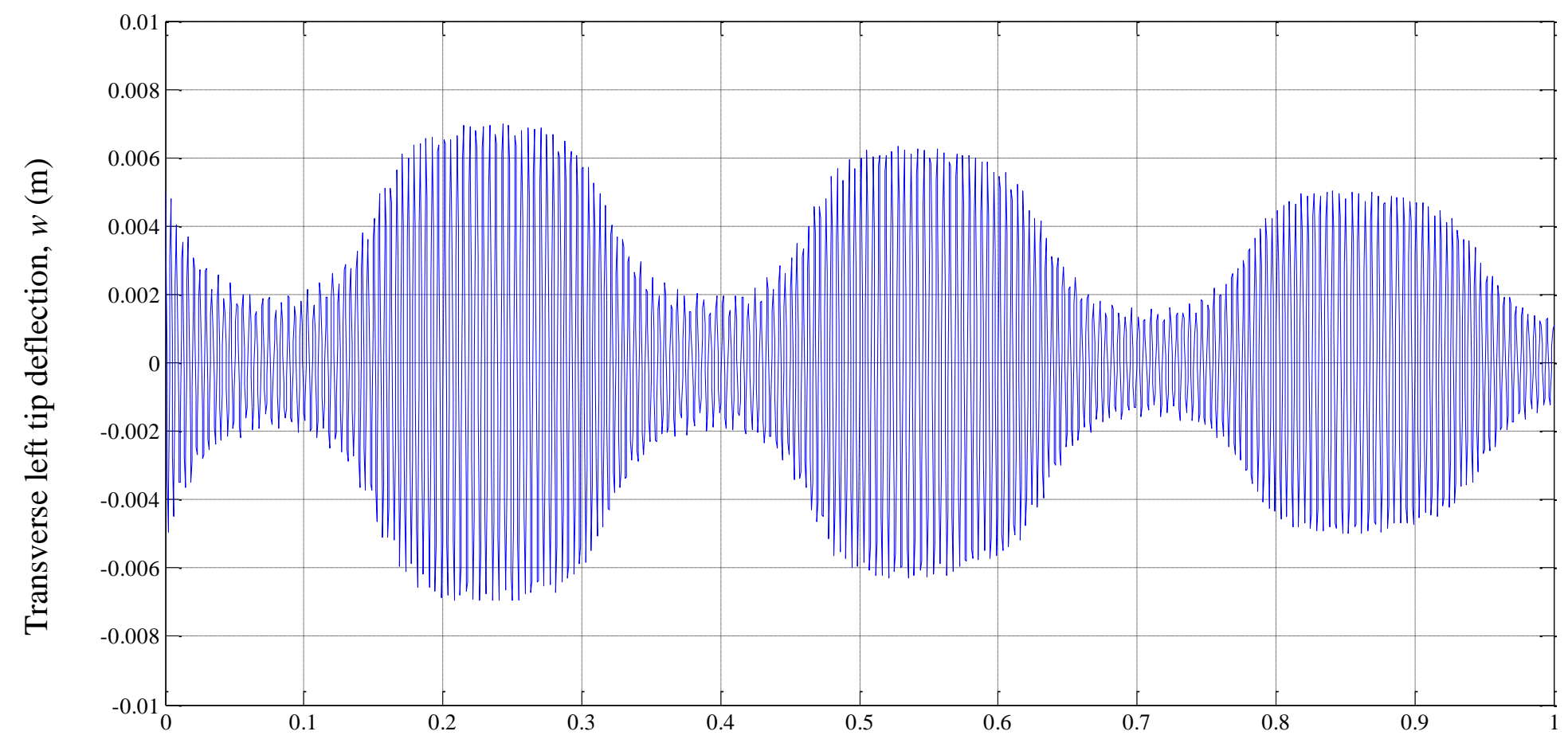

Time (s)

Figure 6.9 Transverse tip deflection at the left end, $w$, CLPT, $\Omega=20 \mathrm{rad} / \mathrm{s},[0 / \pm 45]$ 


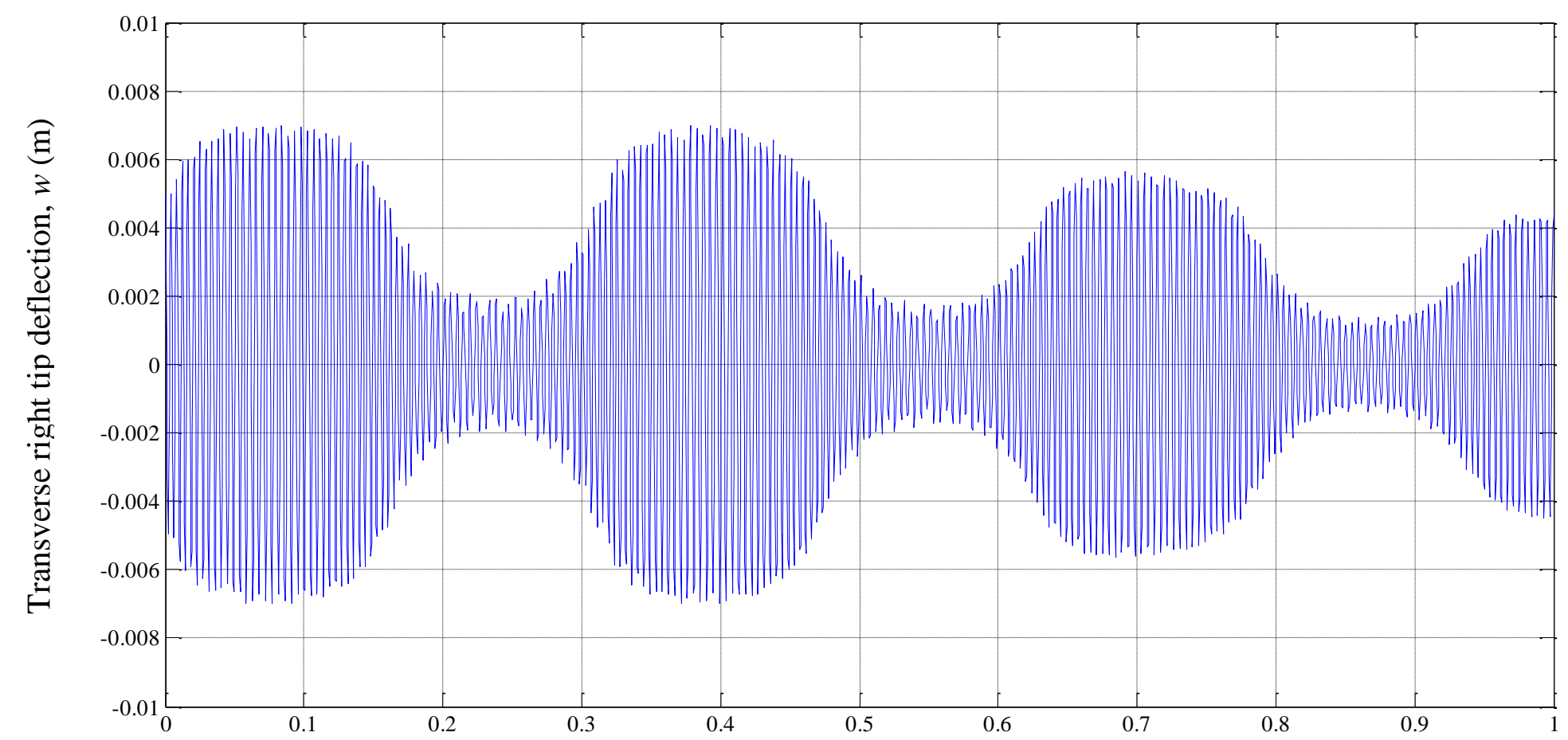

Time (s)

Figure 6.10 Transverse tip deflection at the right end, $w$, CLPT, $\Omega=20 \mathrm{rad} / \mathrm{s},[0 / \pm 45]$ 


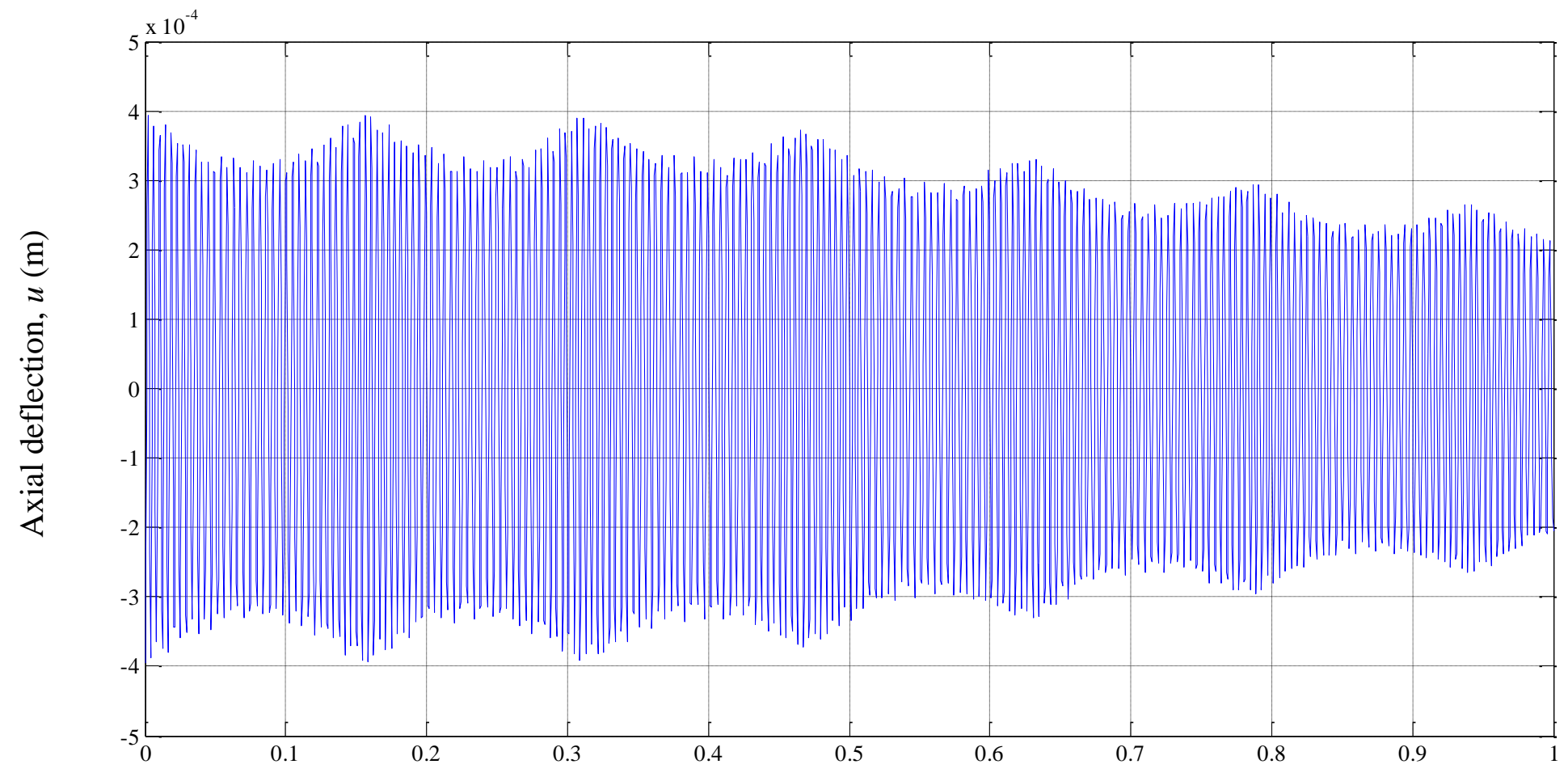

Time (s)

Figure 6.11 Axial deflection at the right end, $u, C L P T, \Omega=20 \mathrm{rad} / \mathrm{s},[0 / \pm 45]$ 
Table 6.7 Transverse tip deflection at the left end, $w$, CLPT, $\Omega=20 \mathrm{rad} / \mathrm{s},[0 / \pm 45]$

\begin{tabular}{|c|c|c|c|c|r|r|r|}
\hline$t(\mathrm{~s})$ & $w(\mathrm{~m})$ & $t(\mathrm{~s})$ & $w(\mathrm{~m})$ & $t(\mathrm{~s})$ & $w(\mathrm{~m})$ & $t(\mathrm{~s})$ & \multicolumn{1}{|c|}{$w(\mathrm{~m})$} \\
\hline 0.000000 & 0.005000 & 0.243000 & 0.006970 & 0.494500 & -0.005991 & 0.746500 & 0.001830 \\
\hline 0.016500 & 0.003692 & 0.254500 & -0.006957 & 0.505500 & 0.006225 & 0.757500 & -0.002188 \\
\hline 0.020500 & 0.003062 & 0.261500 & 0.006867 & 0.512000 & -0.006221 & 0.768500 & 0.002613 \\
\hline 0.038000 & 0.002584 & 0.270500 & 0.006889 & 0.528000 & 0.006334 & 0.778500 & -0.003147 \\
\hline 0.047000 & 0.002359 & 0.281500 & -0.006702 & 0.535000 & -0.006277 & 0.789000 & 0.003903 \\
\hline 0.054000 & -0.002179 & 0.290000 & -0.006283 & 0.549000 & -0.006267 & 0.799500 & -0.004322 \\
\hline 0.061000 & 0.002010 & 0.303000 & -0.005820 & 0.556000 & 0.006258 & 0.805500 & 0.004610 \\
\hline 0.075000 & 0.001903 & 0.311000 & -0.005069 & 0.563000 & -0.006212 & 0.818500 & 0.004954 \\
\hline 0.088500 & 0.001943 & 0.321500 & 0.004039 & 0.570000 & 0.006098 & 0.827500 & 0.004947 \\
\hline 0.095500 & -0.002031 & 0.331500 & -0.003397 & 0.583500 & 0.005995 & 0.836500 & 0.004992 \\
\hline 0.109500 & -0.002181 & 0.342500 & 0.002972 & 0.592500 & 0.005889 & 0.841000 & 0.005023 \\
\hline 0.118500 & -0.002502 & 0.351000 & 0.002491 & 0.605500 & 0.005560 & 0.855000 & 0.004982 \\
\hline 0.129500 & 0.002867 & 0.360000 & 0.002355 & 0.614000 & 0.005207 & 0.862000 & -0.004987 \\
\hline 0.138000 & 0.003280 & 0.376000 & -0.002099 & 0.620000 & -0.004754 & 0.876000 & -0.004958 \\
\hline 0.142000 & 0.003792 & 0.383000 & 0.002040 & 0.630500 & 0.004148 & 0.883000 & 0.004890 \\
\hline 0.158500 & 0.005117 & 0.390000 & -0.001985 & 0.643000 & 0.003075 & 0.892000 & 0.004819 \\
\hline 0.169000 & -0.005968 & 0.409000 & -0.001956 & 0.655500 & 0.002478 & 0.901000 & 0.004677 \\
\hline 0.179500 & 0.006364 & 0.418000 & -0.002065 & 0.666500 & -0.002031 & 0.910000 & 0.004571 \\
\hline 0.181500 & -0.006581 & 0.424500 & 0.002204 & 0.673000 & 0.001808 & 0.925000 & -0.004206 \\
\hline 0.199000 & -0.006864 & 0.433500 & 0.002495 & 0.682000 & 0.001699 & 0.931000 & 0.003891 \\
\hline 0.208000 & -0.006967 & 0.442500 & 0.002850 & 0.696000 & 0.001617 & 0.941500 & -0.003495 \\
\hline 0.215000 & 0.006963 & 0.455000 & 0.003490 & 0.703000 & -0.001589 & 0.954000 & -0.002556 \\
\hline 0.226500 & -0.006936 & 0.469500 & -0.004634 & 0.717000 & -0.001577 & 0.960000 & 0.002256 \\
\hline 0.236000 & -0.006959 & 0.478000 & -0.005143 & 0.724000 & 0.001613 & 0.970500 & -0.001742 \\
\hline 0.000000 & 0.005000 & 0.484000 & 0.005674 & 0.738000 & 0.001710 & 0.988500 & -0.001449 \\
\hline
\end{tabular}




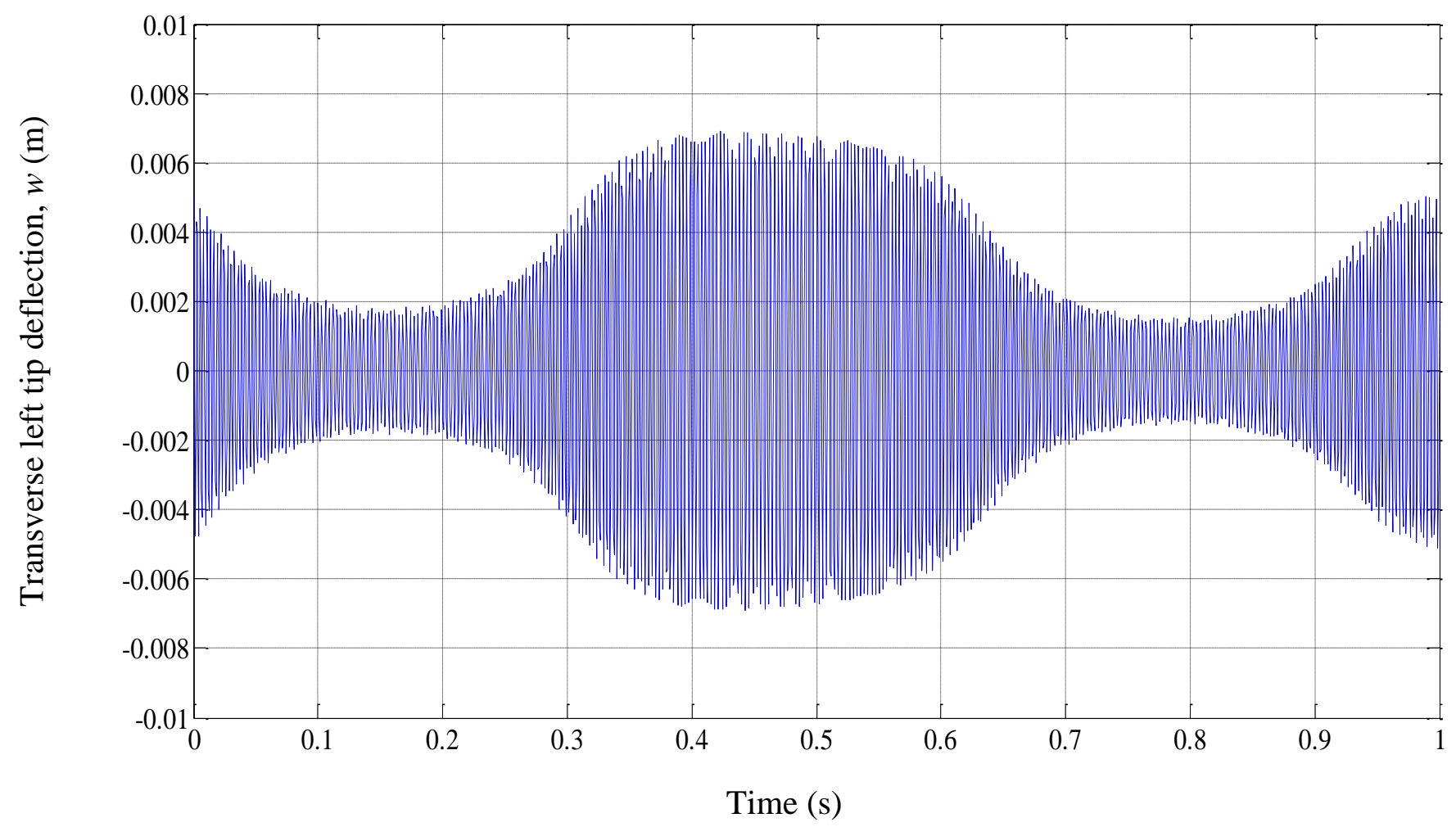

Figure 6.12 Transverse tip deflection at the left end, $w, C L P T, \Omega=10 \mathrm{rad} / \mathrm{s},[0 / \pm 20]$ 


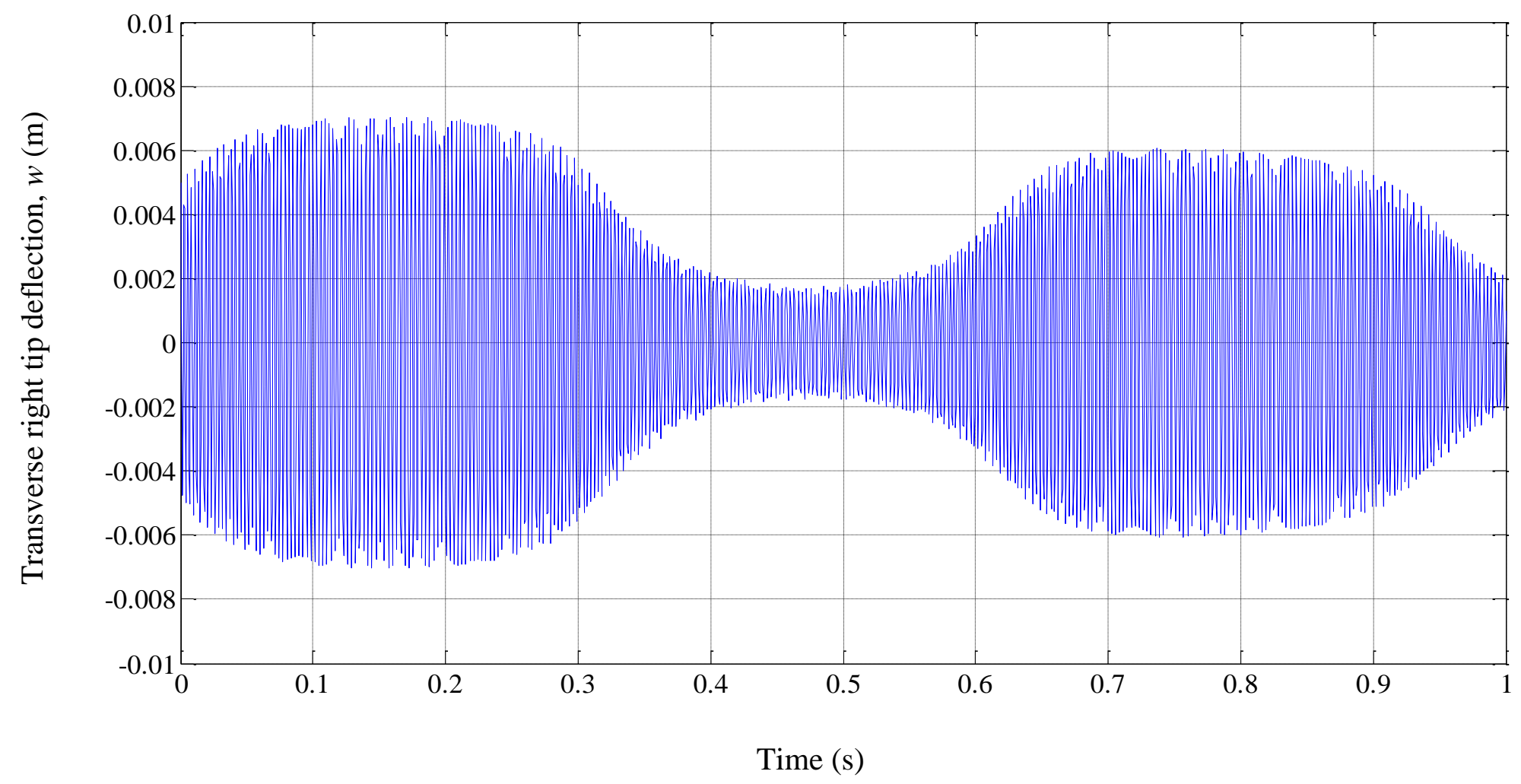

Figure 6.13 Transverse tip deflection at the right end, $w$, CLPT, $\Omega=10 \mathrm{rad} / \mathrm{s},[0 / \pm 20]$ 


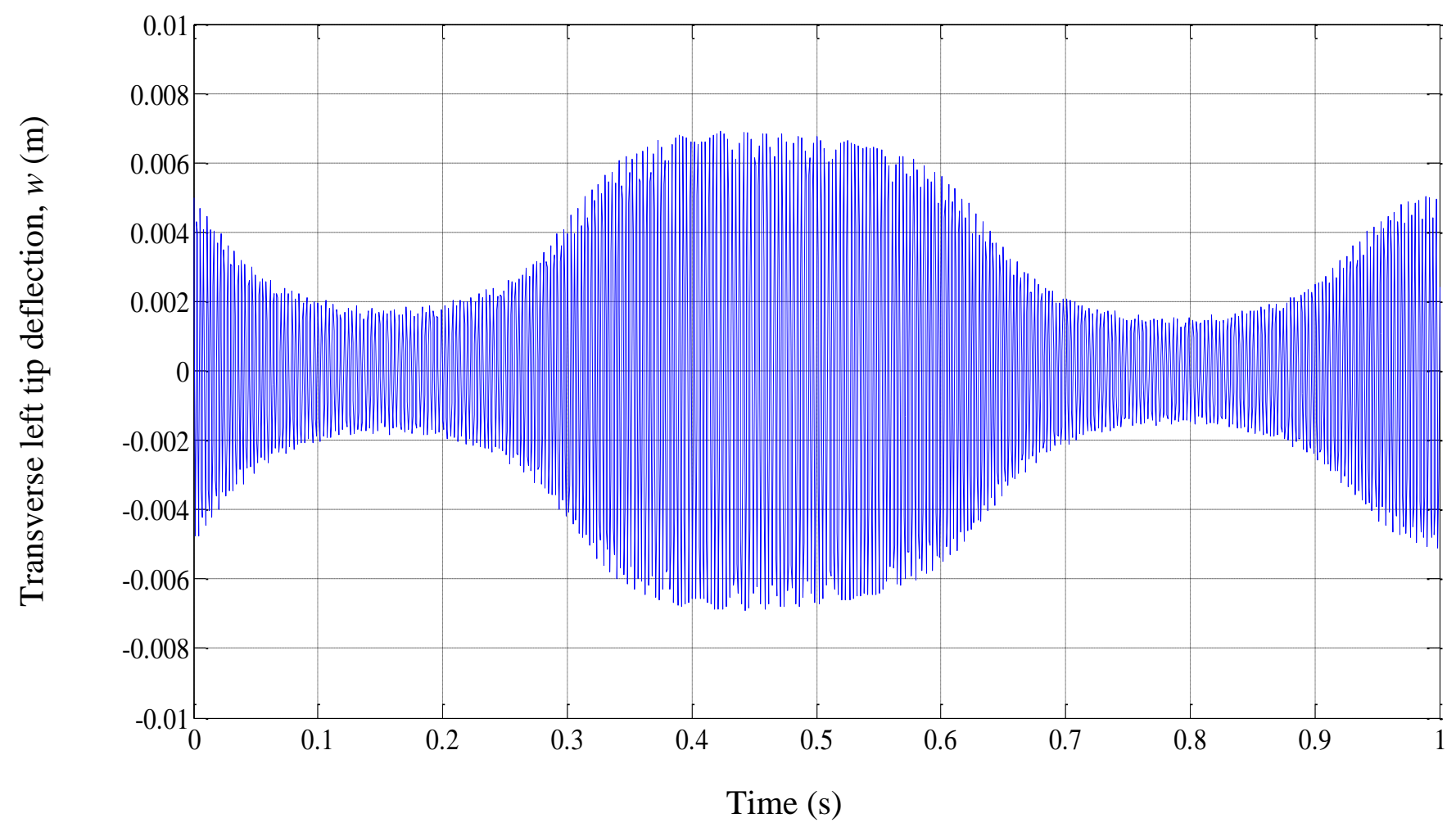

Figure 6.14 Transverse tip deflection at the left end, $w, C L P T, \Omega=10 \mathrm{rad} / \mathrm{s},[0 / \pm 20]$ 
Table 6.8 Transverse tip deflection at the left end, $w$, CLPT, $\Omega=10 \mathrm{rad} / \mathrm{s},[0 / \pm 20]$

\begin{tabular}{|c|c|c|c|c|c|c|c|}
\hline$t(\mathrm{~s})$ & $w(\mathrm{~m})$ & $t(\mathrm{~s})$ & $w(\mathrm{~m})$ & $t(\mathrm{~s})$ & $w(\mathrm{~m})$ & $t(\mathrm{~s})$ & $w(\mathrm{~m})$ \\
\hline 0.000000 & 0.005000 & 0.254000 & -0.002683 & 0.500500 & 0.006758 & 0.758500 & 0.001618 \\
\hline 0.011000 & 0.004460 & 0.267000 & 0.002943 & 0.519000 & 0.006568 & 0.760000 & -0.001524 \\
\hline 0.020500 & -0.004002 & 0.279500 & -0.003268 & 0.525000 & 0.006635 & 0.772500 & -0.001571 \\
\hline 0.033000 & 0.003457 & 0.286500 & 0.003623 & 0.531000 & 0.006529 & 0.785000 & -0.001525 \\
\hline 0.040000 & -0.003265 & 0.299000 & -0.004173 & 0.546000 & 0.006467 & 0.799000 & 0.001552 \\
\hline 0.050000 & 0.002759 & 0.308500 & 0.004666 & 0.550500 & -0.006411 & 0.803500 & -0.001530 \\
\hline 0.060000 & -0.002656 & 0.319500 & 0.005219 & 0.568000 & -0.006196 & 0.817500 & 0.001595 \\
\hline 0.074500 & -0.002392 & 0.329000 & -0.005597 & 0.578000 & 0.006091 & 0.822000 & -0.001528 \\
\hline 0.080500 & -0.002264 & 0.334500 & -0.005812 & 0.586500 & 0.005929 & 0.836000 & 0.001664 \\
\hline 0.091000 & 0.002106 & 0.347000 & 0.006166 & 0.593500 & -0.005769 & 0.846500 & -0.001747 \\
\hline 0.101500 & -0.002047 & 0.354000 & -0.006294 & 0.600500 & 0.005612 & 0.858500 & -0.001842 \\
\hline 0.110500 & -0.001938 & 0.362500 & -0.006461 & 0.611500 & 0.005248 & 0.869000 & 0.001922 \\
\hline 0.126000 & -0.001857 & 0.372500 & 0.006657 & 0.622500 & 0.004850 & 0.879000 & -0.001995 \\
\hline 0.130500 & 0.001877 & 0.388500 & -0.006739 & 0.632000 & -0.004348 & 0.882000 & -0.002197 \\
\hline 0.144500 & -0.001810 & 0.391500 & -0.006807 & 0.643000 & -0.003882 & 0.899000 & -0.002395 \\
\hline 0.157000 & -0.001841 & 0.402000 & 0.006589 & 0.650000 & 0.003571 & 0.909000 & 0.002733 \\
\hline 0.169500 & -0.001758 & 0.418500 & -0.006856 & 0.661000 & 0.003150 & 0.914500 & 0.002963 \\
\hline 0.171000 & 0.001840 & 0.423000 & 0.006922 & 0.676500 & -0.002667 & 0.927000 & -0.003339 \\
\hline 0.189500 & 0.001870 & 0.438500 & 0.006574 & 0.683500 & 0.002388 & 0.936500 & 0.003737 \\
\hline 0.194000 & -0.001785 & 0.443000 & -0.006899 & 0.691000 & -0.002141 & 0.947500 & 0.004145 \\
\hline 0.208000 & 0.002035 & 0.458500 & -0.006858 & 0.702500 & -0.002126 & 0.958500 & 0.004448 \\
\hline 0.218500 & -0.002108 & 0.460000 & 0.006851 & 0.713000 & 0.001889 & 0.969500 & 0.004783 \\
\hline 0.229000 & 0.002240 & 0.472500 & 0.006840 & 0.729500 & -0.001758 & 0.975000 & 0.004864 \\
\hline 0.235000 & 0.002341 & 0.486500 & -0.006809 & 0.734000 & 0.001724 & 0.989000 & 0.005010 \\
\hline 0.241000 & 0.002365 & 0.499000 & -0.006671 & 0.740000 & 0.001711 & 0.999000 & -0.005117 \\
\hline
\end{tabular}




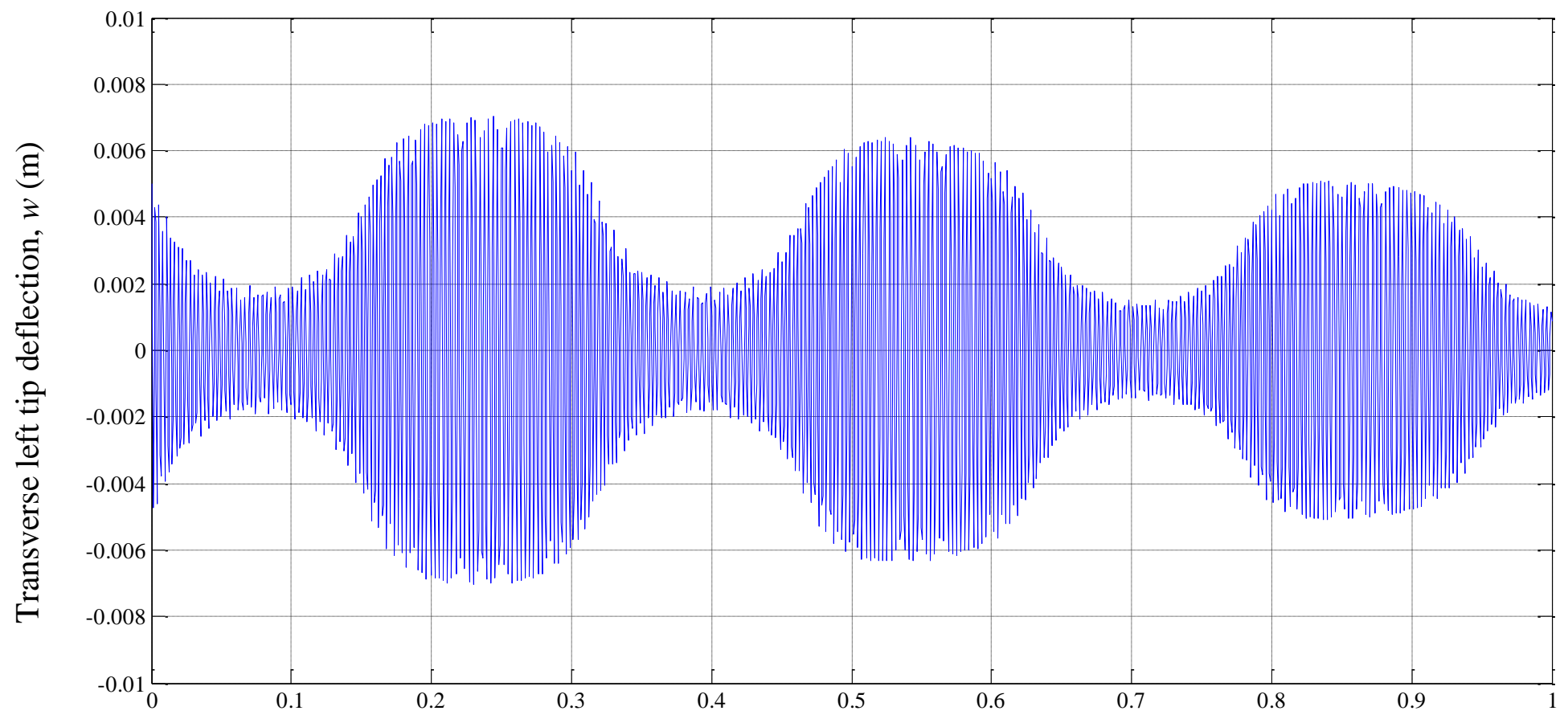

Time (s)

Figure 6.15 Transverse Tip deflection at the left end, $w$, CLPT, $\Omega=20 \mathrm{rad} / \mathrm{sec},[0 / \pm 20]$ 


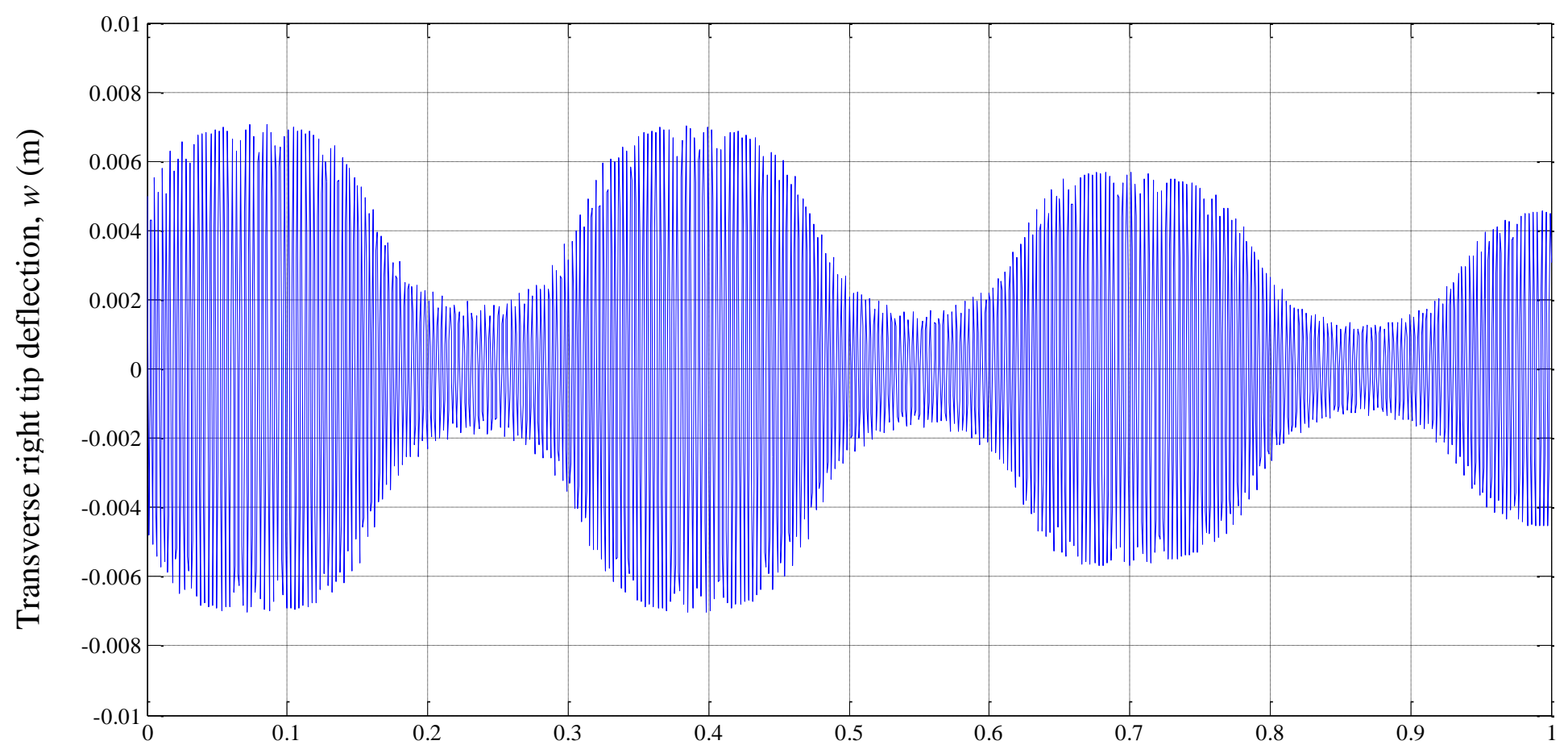

Time (s)

Figure 6.16 Transverse tip deflection at the right end, $w$, CLPT, $\Omega=20 \mathrm{rad} / \mathrm{s},[0 / \pm 20]$ 


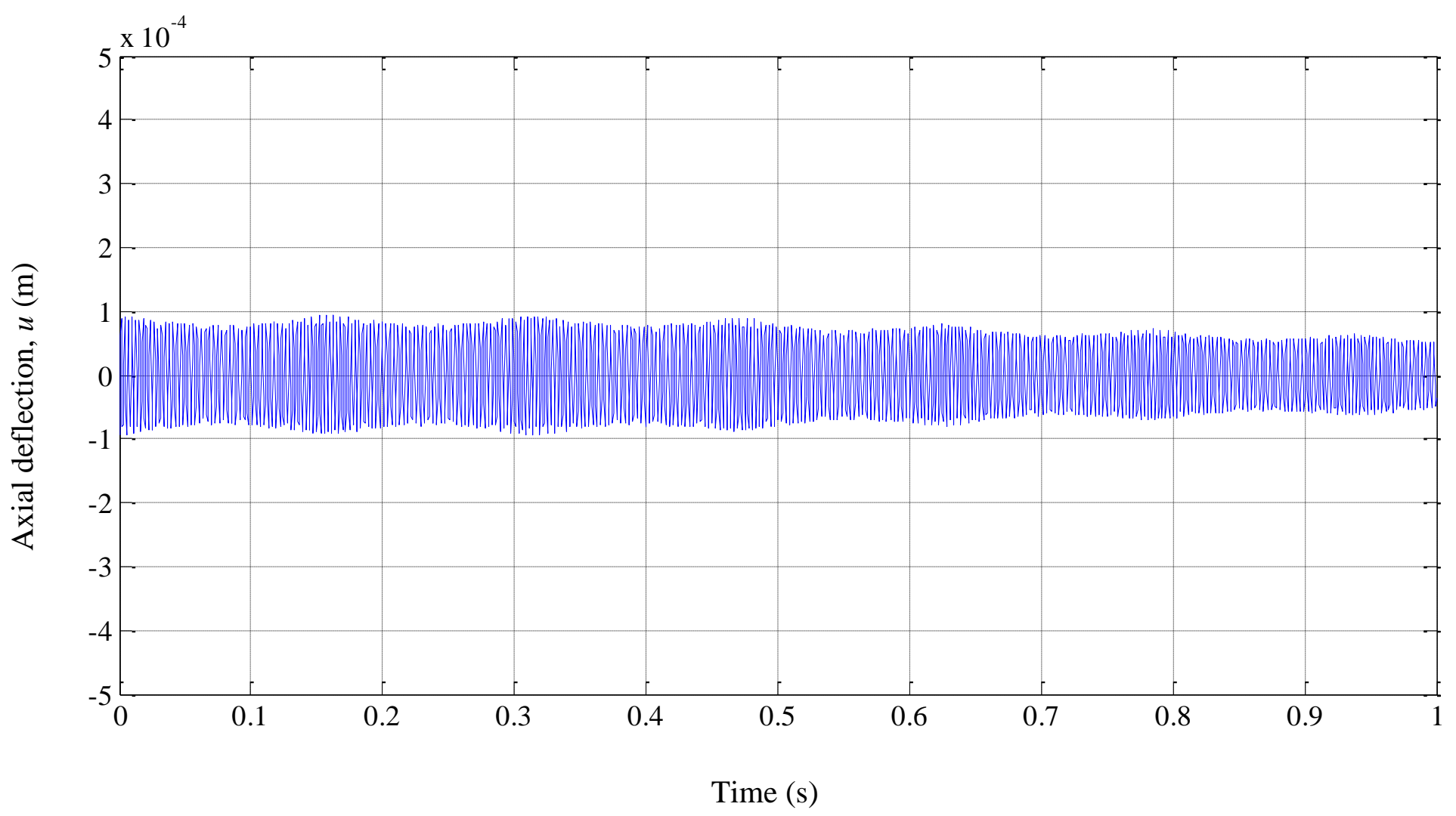

Figure 6.17 Axial deflection at the right end, $u, C L P T, \Omega=20 \mathrm{rad} / \mathrm{s},[0 / \pm 20]$ 
Table 6.9 Transverse tip deflection at the left end, $w$, CLPT, $\Omega=20 \mathrm{rad} / \mathrm{s},[0 / \pm 20]$

\begin{tabular}{|c|c|c|c|c|c|c|r|}
\hline$t(\mathrm{~s})$ & $w(\mathrm{~m})$ & $t(\mathrm{~s})$ & $w(\mathrm{~m})$ & $t(\mathrm{~s})$ & $w(\mathrm{~m})$ & $t(\mathrm{~s})$ & $w(\mathrm{~m})$ \\
\hline 0.000000 & 0.005000 & 0.258000 & -0.006979 & 0.507500 & -0.006275 & 0.758500 & 0.002119 \\
\hline 0.011000 & 0.004031 & 0.262500 & 0.006976 & 0.519500 & -0.006322 & 0.768500 & -0.002661 \\
\hline 0.022000 & 0.003066 & 0.273000 & -0.006855 & 0.524000 & 0.006390 & 0.779500 & -0.003254 \\
\hline 0.035000 & -0.002566 & 0.280500 & 0.006557 & 0.530000 & 0.006195 & 0.786500 & 0.003858 \\
\hline 0.041000 & -0.002363 & 0.290500 & -0.006385 & 0.542500 & 0.006382 & 0.796000 & -0.004480 \\
\hline 0.051500 & 0.002148 & 0.303000 & 0.005973 & 0.556500 & -0.006316 & 0.808500 & 0.004701 \\
\hline 0.060500 & 0.001872 & 0.310000 & -0.005358 & 0.569000 & -0.006059 & 0.811500 & 0.004854 \\
\hline 0.070000 & 0.001946 & 0.321000 & -0.004232 & 0.575000 & -0.006175 & 0.824500 & -0.005051 \\
\hline 0.088500 & 0.001923 & 0.330500 & 0.003630 & 0.581000 & -0.006021 & 0.835000 & 0.005084 \\
\hline 0.096500 & -0.001876 & 0.340500 & -0.002688 & 0.590000 & -0.005971 & 0.841000 & 0.005073 \\
\hline 0.107000 & 0.002181 & 0.352000 & -0.002495 & 0.604500 & -0.005649 & 0.856500 & 0.005057 \\
\hline 0.117500 & -0.002410 & 0.362500 & 0.002201 & 0.611500 & 0.005412 & 0.869000 & 0.005013 \\
\hline 0.123500 & -0.002552 & 0.373000 & -0.002006 & 0.622500 & 0.004719 & 0.870500 & -0.005014 \\
\hline 0.139000 & 0.003448 & 0.387000 & 0.001925 & 0.632000 & -0.003842 & 0.886000 & -0.004943 \\
\hline 0.147500 & 0.004136 & 0.399500 & 0.001910 & 0.640500 & -0.003202 & 0.890500 & 0.004899 \\
\hline 0.157000 & -0.005138 & 0.409000 & 0.001850 & 0.650000 & 0.002515 & 0.902500 & 0.004779 \\
\hline 0.168000 & -0.005964 & 0.419500 & -0.002060 & 0.663000 & -0.002079 & 0.910000 & -0.004473 \\
\hline 0.175000 & 0.006224 & 0.428500 & -0.002196 & 0.673500 & 0.001782 & 0.920000 & 0.004377 \\
\hline 0.182000 & -0.006524 & 0.434500 & -0.002425 & 0.684000 & -0.001619 & 0.931000 & 0.004028 \\
\hline 0.196500 & -0.006859 & 0.449000 & -0.002988 & 0.698000 & 0.001516 & 0.942000 & 0.003427 \\
\hline 0.207000 & 0.006944 & 0.456000 & 0.003465 & 0.702500 & -0.001451 & 0.951500 & -0.002676 \\
\hline 0.211500 & -0.006992 & 0.467000 & 0.004421 & 0.712000 & -0.001509 & 0.960000 & -0.002299 \\
\hline 0.228500 & 0.006979 & 0.476500 & -0.005298 & 0.726000 & 0.001514 & 0.970000 & 0.001675 \\
\hline 0.230000 & -0.007037 & 0.487500 & -0.005823 & 0.735000 & 0.001644 & 0.980000 & -0.001592 \\
\hline 0.244000 & 0.007034 & 0.496000 & -0.006049 & 0.747000 & 0.001872 & 0.990500 & 0.001380 \\
\hline
\end{tabular}




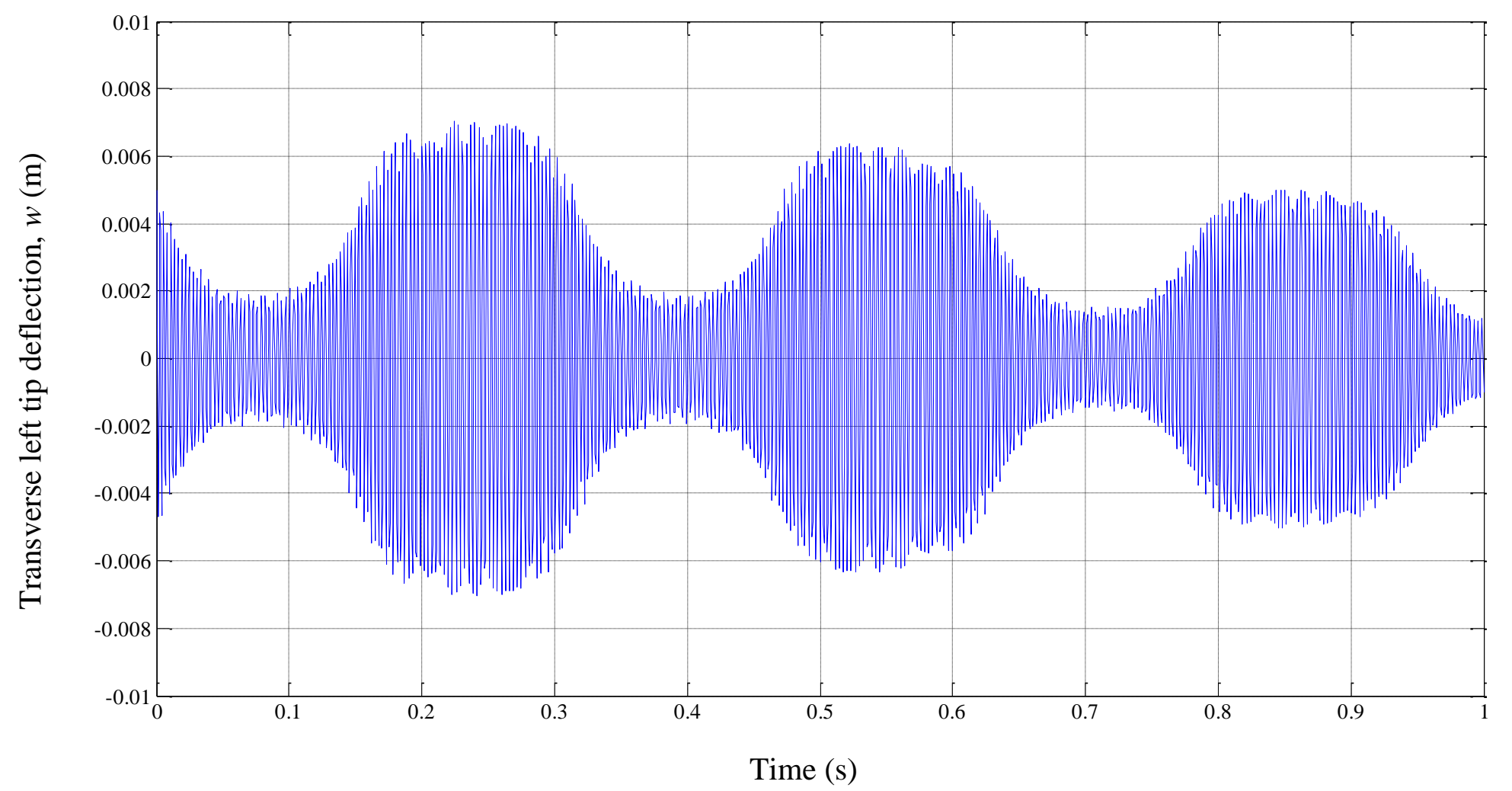

Figure 6.18 Transverse tip deflection at the left end, $w$, CLPT, $\Omega=20 \mathrm{rad} / \mathrm{s},[0 / \pm 45]_{\mathrm{s}}$ 


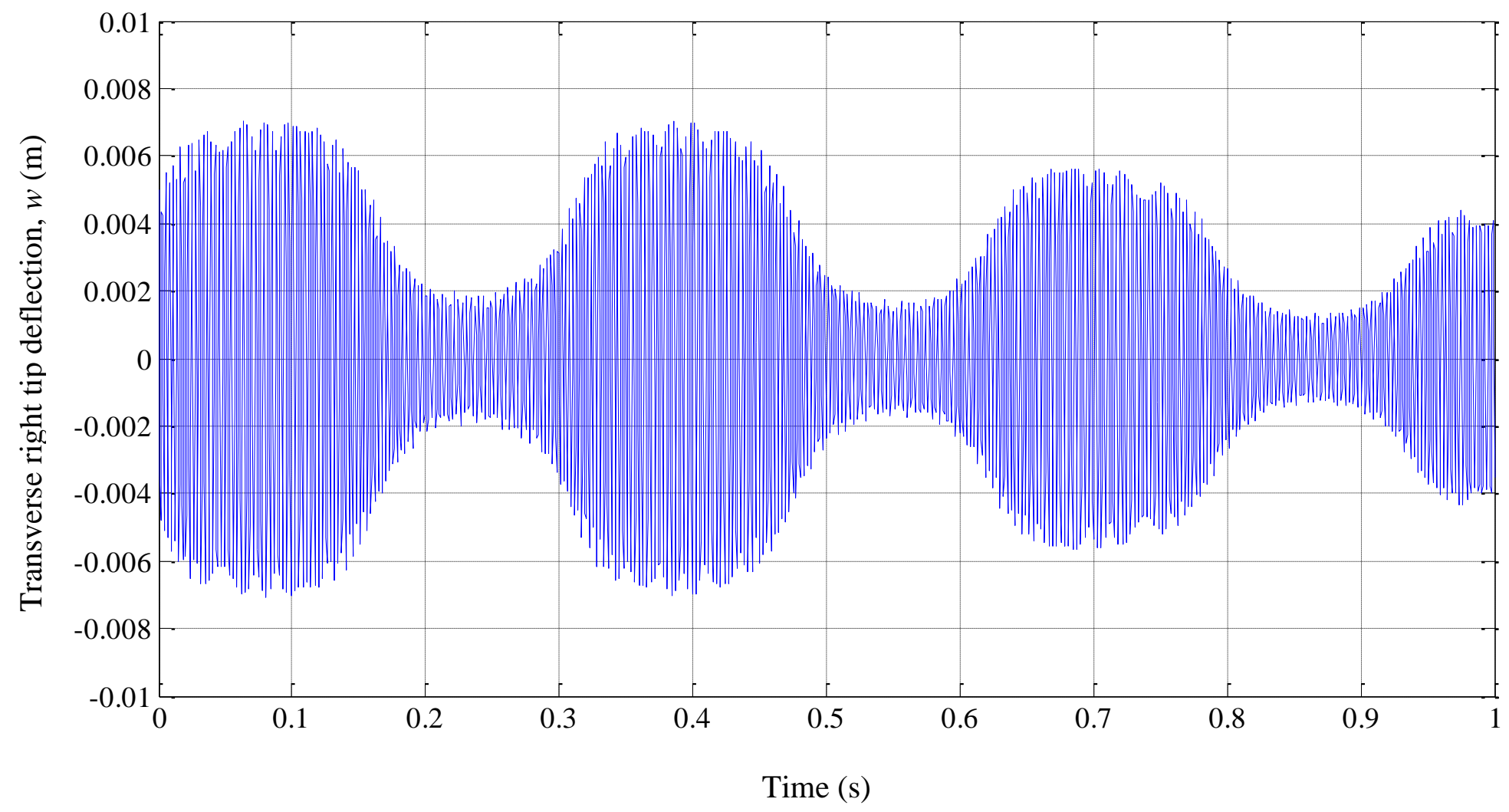

Figure 6.19 Transverse tip deflection at the right end, $w$, CLPT, $\Omega=20 \mathrm{rad} / \mathrm{s},[0 / \pm 45]_{\mathrm{s}}$ 


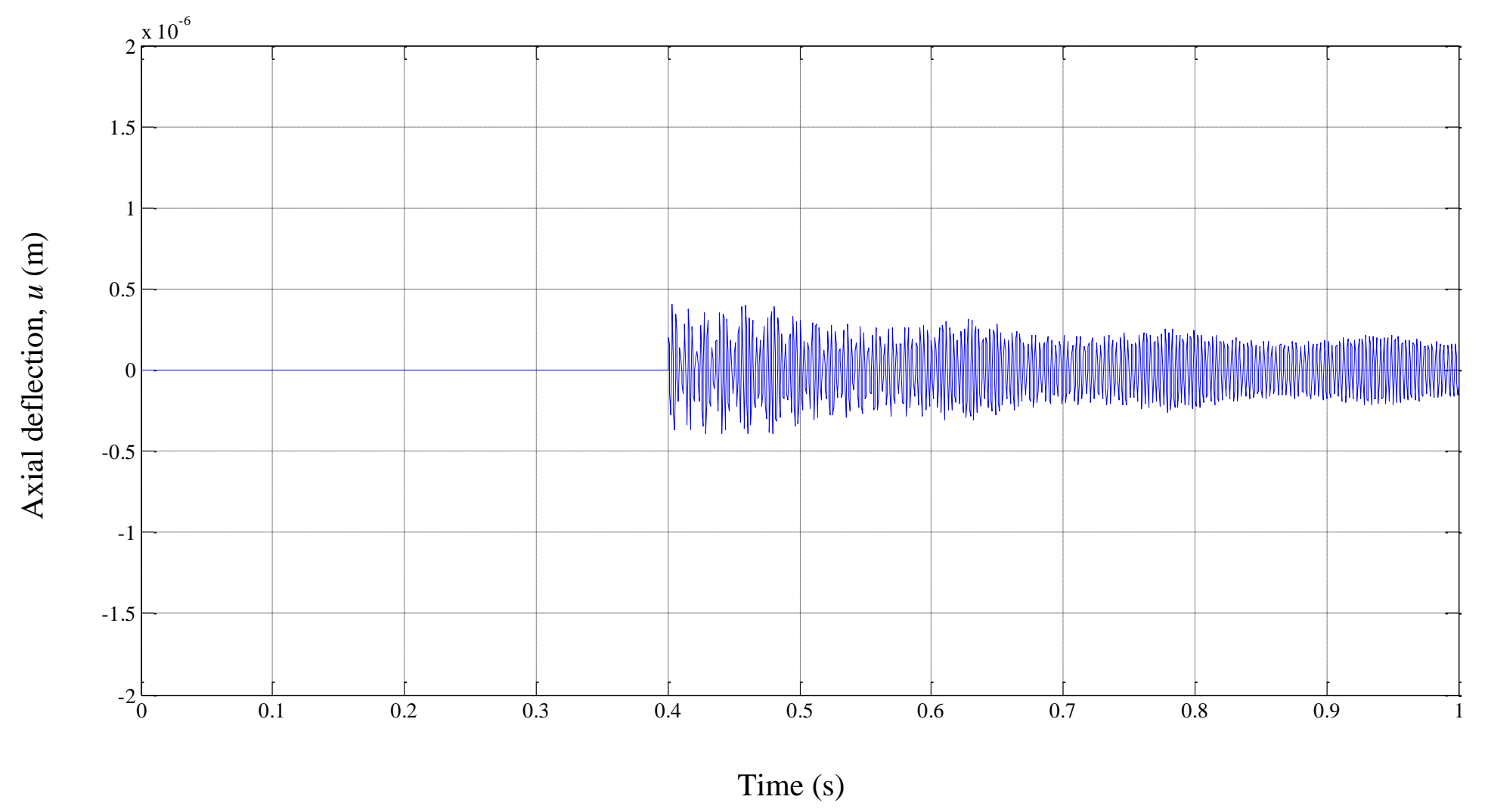

Figure 6.20 Axial deflection at the right end, $u, C L P T, \Omega=20 \mathrm{rad} / \mathrm{s},[0 / \pm 45]_{\mathrm{s}}$ 
Table 6.10 Transverse tip deflection at the left end, $w, C L P T, \Omega=20 \mathrm{rad} / \mathrm{s},[0 / \pm 45]_{\mathrm{s}}$

\begin{tabular}{|c|r|r|r|r|r|r|r|}
\hline$t(\mathrm{~s})$ & $w(\mathrm{~m})$ & $t(\mathrm{~s})$ & $w(\mathrm{~m})$ & $t(\mathrm{~s})$ & $w(\mathrm{~m})$ & \multicolumn{1}{c|}{$t(\mathrm{~s})$} & \multicolumn{1}{c|}{$w(\mathrm{~m})$} \\
\hline 0.000000 & 0.005000 & 0.258500 & 0.006944 & 0.500000 & -0.006042 & 0.753000 & 0.002072 \\
\hline 0.011000 & 0.004034 & 0.260000 & -0.006990 & 0.517500 & -0.006331 & 0.768500 & -0.002663 \\
\hline 0.020500 & -0.003187 & 0.270500 & 0.006890 & 0.522000 & 0.006351 & 0.779500 & -0.003207 \\
\hline 0.033500 & 0.002661 & 0.288000 & 0.006603 & 0.531000 & 0.006111 & 0.786500 & 0.003862 \\
\hline 0.041000 & -0.002082 & 0.295000 & -0.006344 & 0.545000 & -0.006337 & 0.796000 & -0.004431 \\
\hline 0.050000 & -0.001995 & 0.302000 & 0.005947 & 0.559000 & 0.006260 & 0.808500 & 0.004710 \\
\hline 0.065000 & -0.002017 & 0.311500 & -0.005174 & 0.560500 & -0.006205 & 0.818500 & -0.004919 \\
\hline 0.077500 & -0.001873 & 0.322500 & -0.004316 & 0.577500 & 0.005772 & 0.820000 & 0.004914 \\
\hline 0.082000 & 0.001866 & 0.333500 & -0.003346 & 0.582000 & -0.005759 & 0.839500 & -0.004795 \\
\hline 0.096000 & -0.002049 & 0.340500 & 0.002906 & 0.598000 & 0.005714 & 0.848500 & -0.005032 \\
\hline 0.106500 & 0.002114 & 0.353500 & -0.002298 & 0.606500 & 0.005517 & 0.850000 & 0.005009 \\
\hline 0.117000 & -0.002409 & 0.364000 & 0.002148 & 0.613500 & -0.005199 & 0.864000 & -0.004993 \\
\hline 0.128500 & -0.002651 & 0.373000 & 0.001884 & 0.620500 & 0.004618 & 0.879500 & -0.004909 \\
\hline 0.138500 & 0.003183 & 0.382500 & 0.001966 & 0.630000 & -0.003955 & 0.881000 & 0.004937 \\
\hline 0.145500 & -0.003971 & 0.399500 & -0.001941 & 0.641000 & -0.003055 & 0.890000 & 0.004663 \\
\hline 0.156500 & -0.004850 & 0.404000 & 0.001873 & 0.652000 & -0.002555 & 0.906500 & -0.004689 \\
\hline 0.166000 & 0.005694 & 0.418000 & -0.002071 & 0.665000 & 0.002073 & 0.918000 & -0.004442 \\
\hline 0.178500 & -0.006404 & 0.428500 & 0.002285 & 0.671000 & 0.001887 & 0.925000 & 0.004200 \\
\hline 0.187000 & -0.006671 & 0.434500 & 0.002303 & 0.681500 & -0.001685 & 0.930500 & 0.003940 \\
\hline 0.190000 & -0.006530 & 0.448500 & 0.002867 & 0.690500 & -0.001585 & 0.941500 & 0.003353 \\
\hline 0.204500 & -0.006483 & 0.457000 & 0.003585 & 0.704500 & 0.001523 & 0.951000 & -0.002663 \\
\hline 0.218500 & 0.006674 & 0.468000 & 0.004373 & 0.717000 & 0.001513 & 0.963500 & 0.002122 \\
\hline 0.224500 & 0.007023 & 0.479000 & 0.005197 & 0.721500 & -0.001549 & 0.972000 & 0.001775 \\
\hline 0.238500 & -0.006951 & 0.484500 & 0.005686 & 0.736500 & -0.001497 & 0.982500 & -0.001396 \\
\hline 0.241500 & -0.007035 & 0.498500 & 0.006129 & 0.748500 & -0.001825 & 0.994000 & -0.001210 \\
\hline
\end{tabular}




\subsubsection{Moving beam simulation using FSDT}

In this section, the response of the moving beam with FSDT formulation is shown. The laminate lay ups and the axial oscillation frequencies are kept same as those used in CLPT. For the sake of comparison, the gains supplied to reduce the amplitude are same as the ones used for CLPT. It is obseved that for a certain gain, the amplitude reduces more for the beam with FSDT formulation. The left end of the axial displacement is fixed as before. The tip deflections for the left and right end and the axial deflection at the right end of the beam with $[0 / \pm 45]$ lay up and axial oscillation frequency of $10 \mathrm{rad} / \mathrm{s}$ is shown in Figures $6.21-6.27$. The tip deflections at the left end of the beam are presented in Table 6.12. The bending and shear components of the transverse deflection are shown separately and the total transverse deflection is also shown. A similar beat-like phenomenon is observed with slightly different amplitudes.

The response of the beam with [0/ \pm 45$]$ lay up with axial oscillation frequency of $20 \mathrm{rad} / \mathrm{s}$ is shown from Figures $6.28-6.34$. The transverse deflections at the left end of the beam are presented in Table 6.13. The beat period is smaller with increased axial frequency. The response of the beam with $[0 / \pm 20]$ with axial frequencies $10 \mathrm{rad} / \mathrm{sec}$ and $20 \mathrm{rad} / \mathrm{sec}$ is presented from Figures $6.35-6.48$. Tables 6.14 and 6.15 present the tip deflections at the left end of the beam. The results for the $[0 / \pm 45]_{\mathrm{s}}$ laminate beam with axial oscillation frequency of $20 \mathrm{rad} / \mathrm{s}$ is shown in Figures $6.49-6.55$. Table 6.16 presents the tip deflections at the left end of the beam. The magnification factors for the tip deflections due to the axial oscillation for all the different laminate lay ups are summarized in Table 6.11 . 
Table 6.11 Magnification factors for the moving beams with FSDT formulation

\begin{tabular}{|c|c|c|c|c|}
\hline Lay up & frequency & $w_{\max }^{\text {static }}$ & $w_{\max }^{\text {moving }}$ & $\begin{array}{c}\text { Mag. factor } \\
=\frac{w_{\text {moving }}^{\text {movic }}}{w_{\text {mati }}}\end{array}$ \\
\hline$[0 / \pm 20]$ & 10 & 0.005 & 0.006929 & 1.3858 \\
\hline$[0 / \pm 20]$ & 20 & 0.005 & 0.007021 & 1.4042 \\
\hline$[0 / \pm 45]$ & 10 & 0.005 & 0.006907 & 1.3814 \\
\hline$[0 / \pm 45]$ & 20 & 0.005 & 0.0070814 & 1.4163 \\
\hline$[0 / \pm 45]_{\mathrm{s}}$ & 20 & 0.005 & 0.0070133 & 1.4066 \\
\hline
\end{tabular}




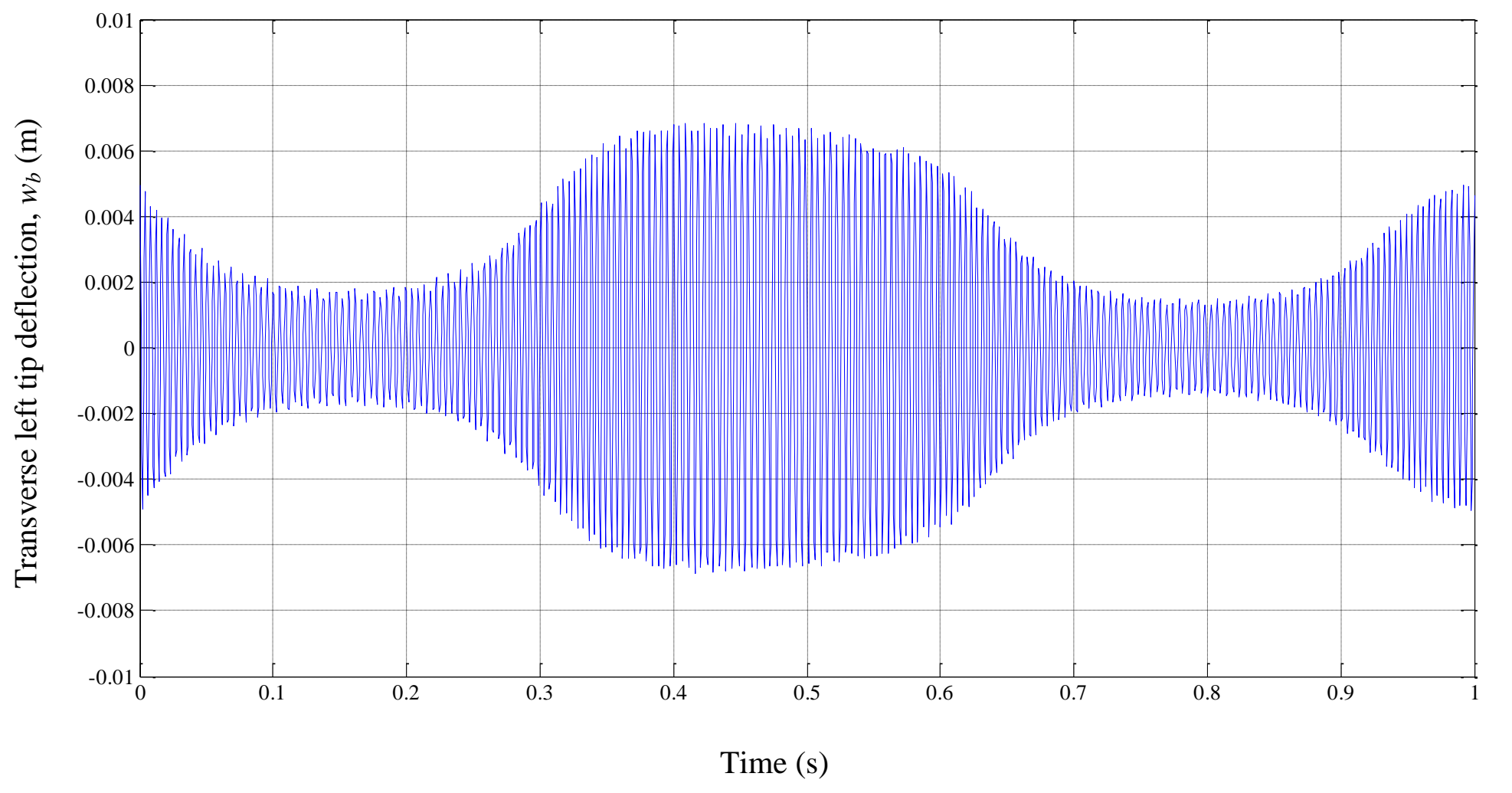

Figure 6.21 Transverse tip deflection at the left end, $w_{b}$, FSDT, $\Omega=10 \mathrm{rad} / \mathrm{s},[0 / \pm 45]$ 


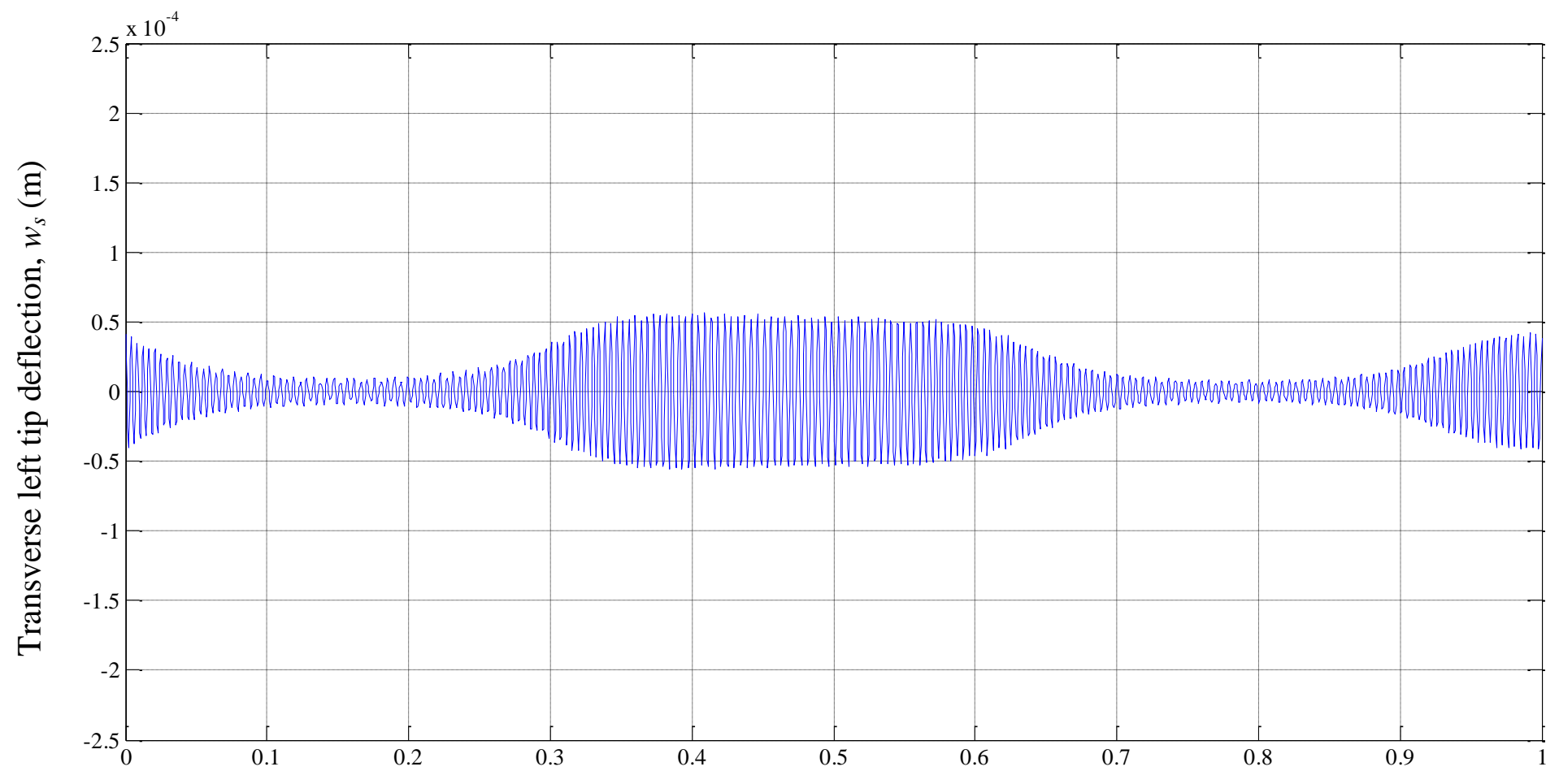

Time (s)

Figure 6.22 Transverse tip deflection at the left end, $w_{s}$, FSDT, $\Omega=10 \mathrm{rad} / \mathrm{s},[0 / \pm 45]$ 


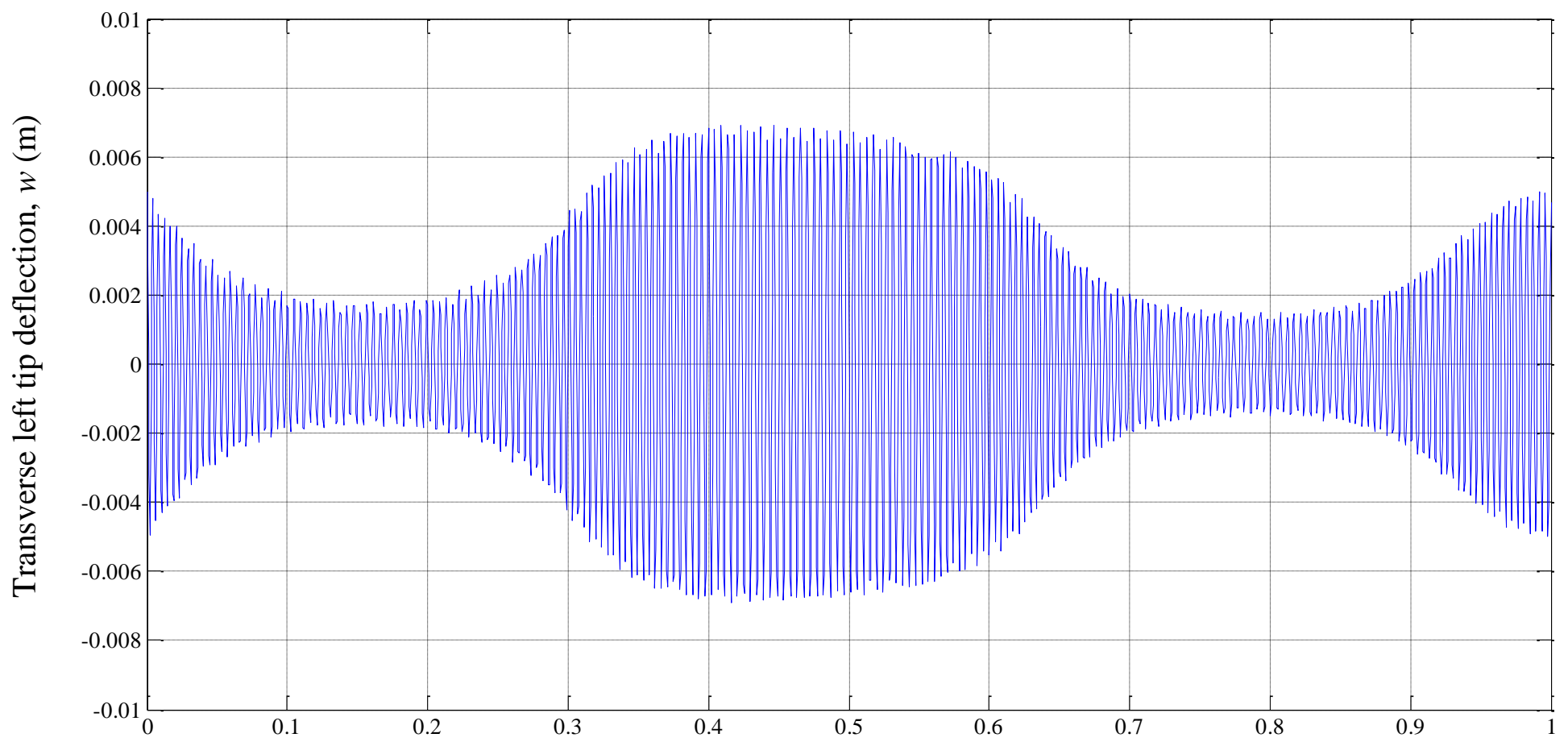

Time (s)

Figure 6.23 Transverse tip deflection at the left end, $w$, FSDT, $\Omega=10 \mathrm{rad} / \mathrm{s},[0 / \pm 45]$ 


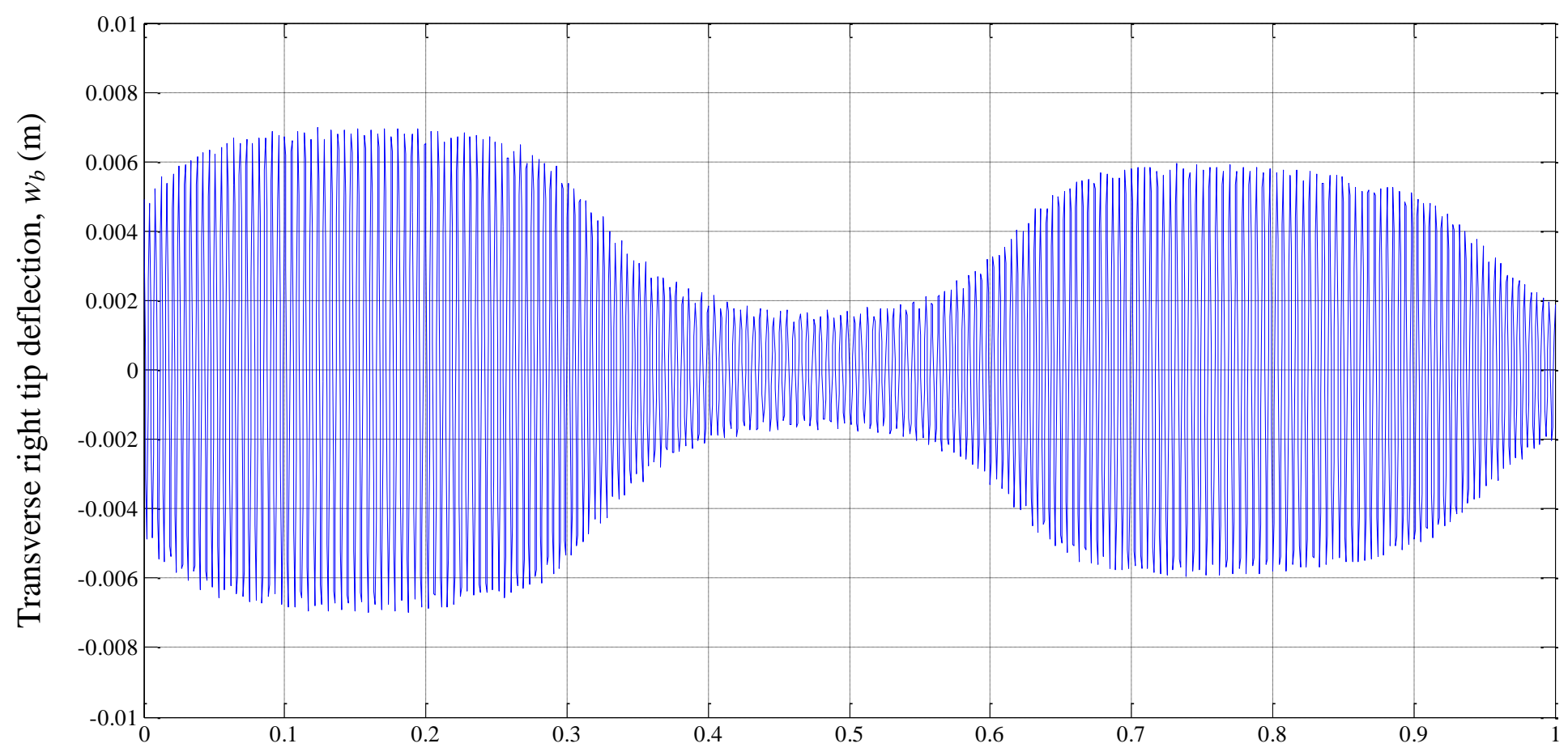

Time (s)

Figure 6.24 Transverse tip deflection at the right end, $w_{b}$, FSDT, $\Omega=10 \mathrm{rad} / \mathrm{s},[0 / \pm 45]$ 


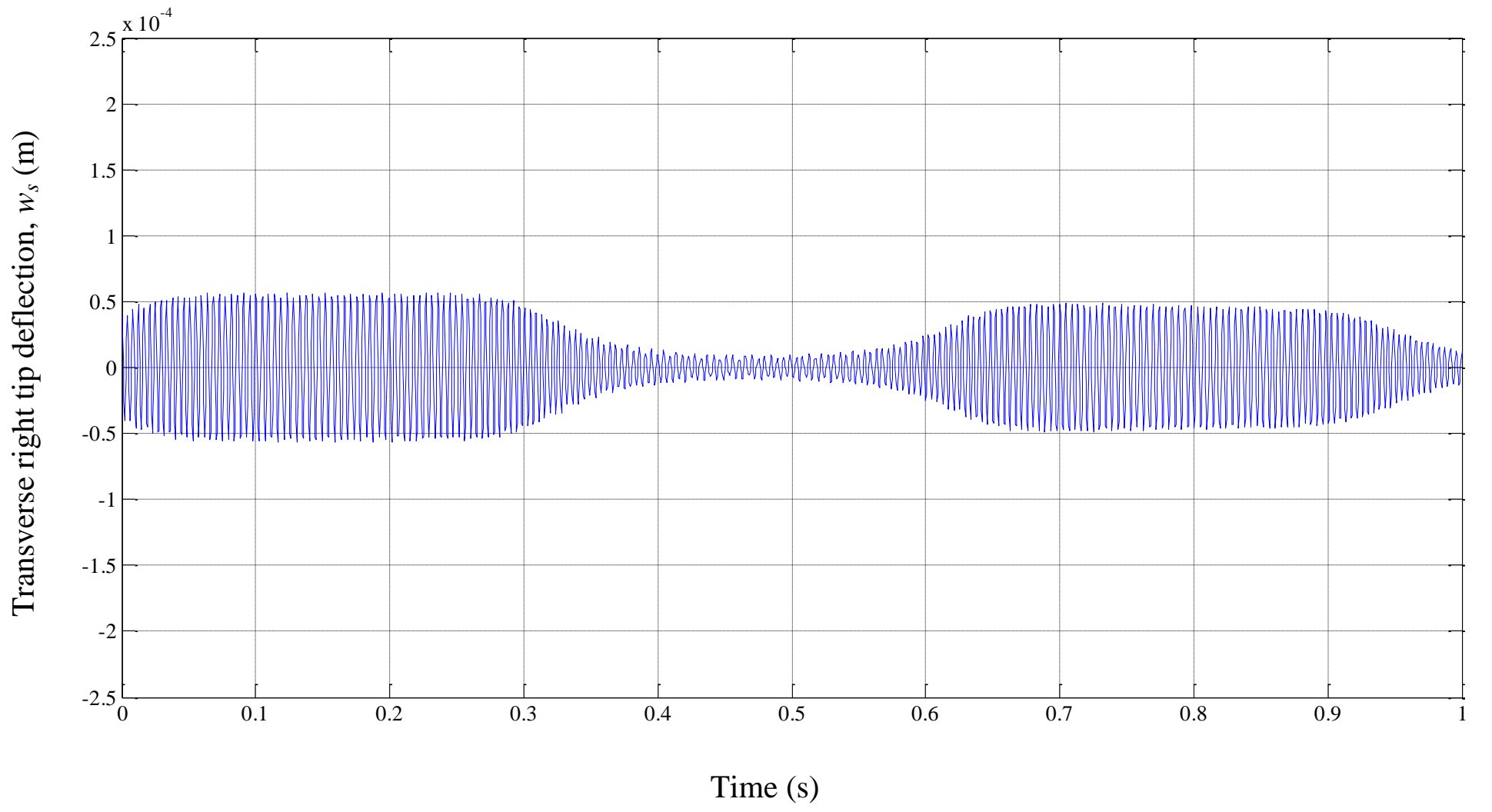

Figure 6.25 Transverse tip deflection at the right end, $w_{s}$, FSDT, $\Omega=10 \mathrm{rad} / \mathrm{s},[0 / \pm 45]$ 


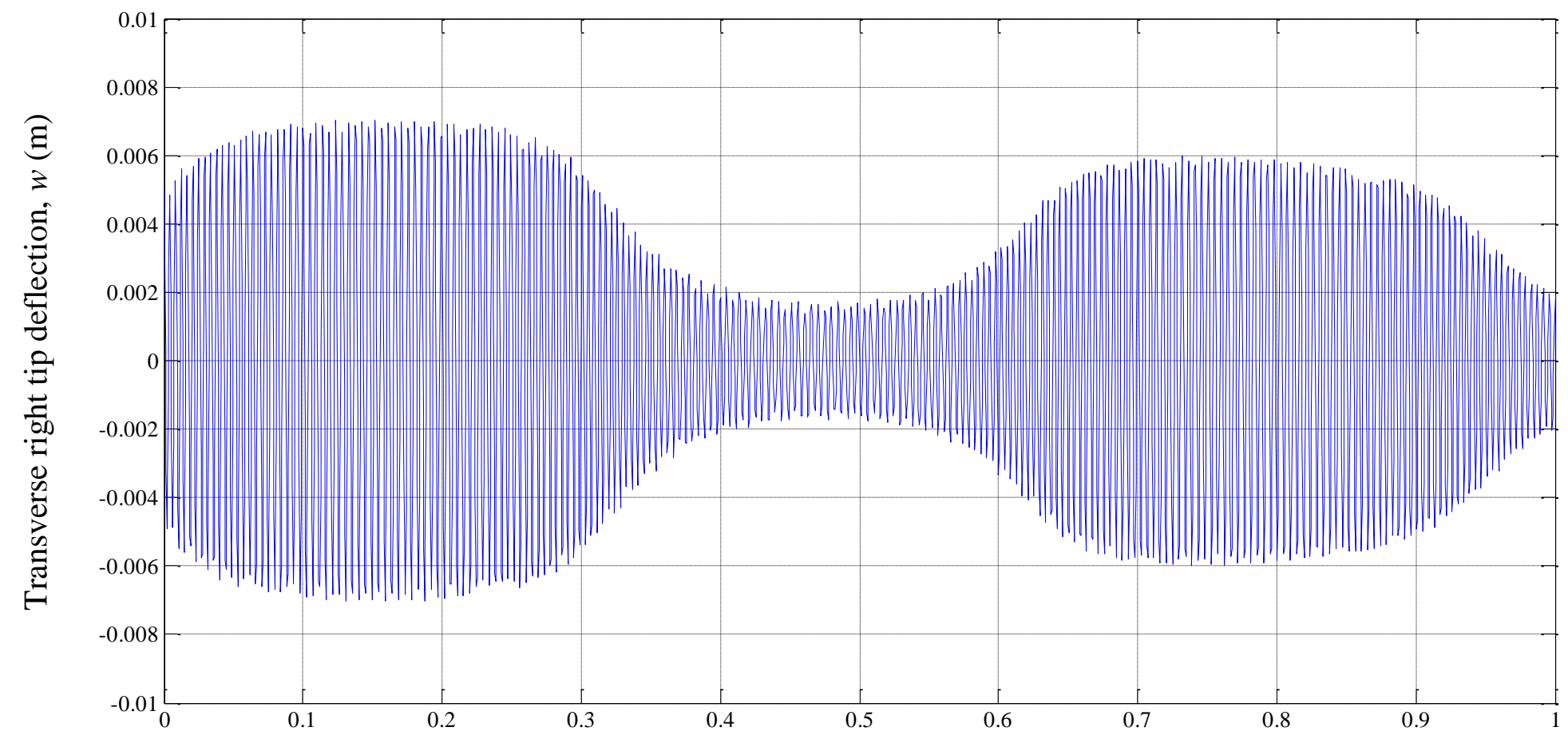

Time (s)

Figure 6.26 Transverse tip deflection at the right end, $w$, FSDT, $\Omega=10 \mathrm{rad} / \mathrm{s},[0 / \pm 45]$ 


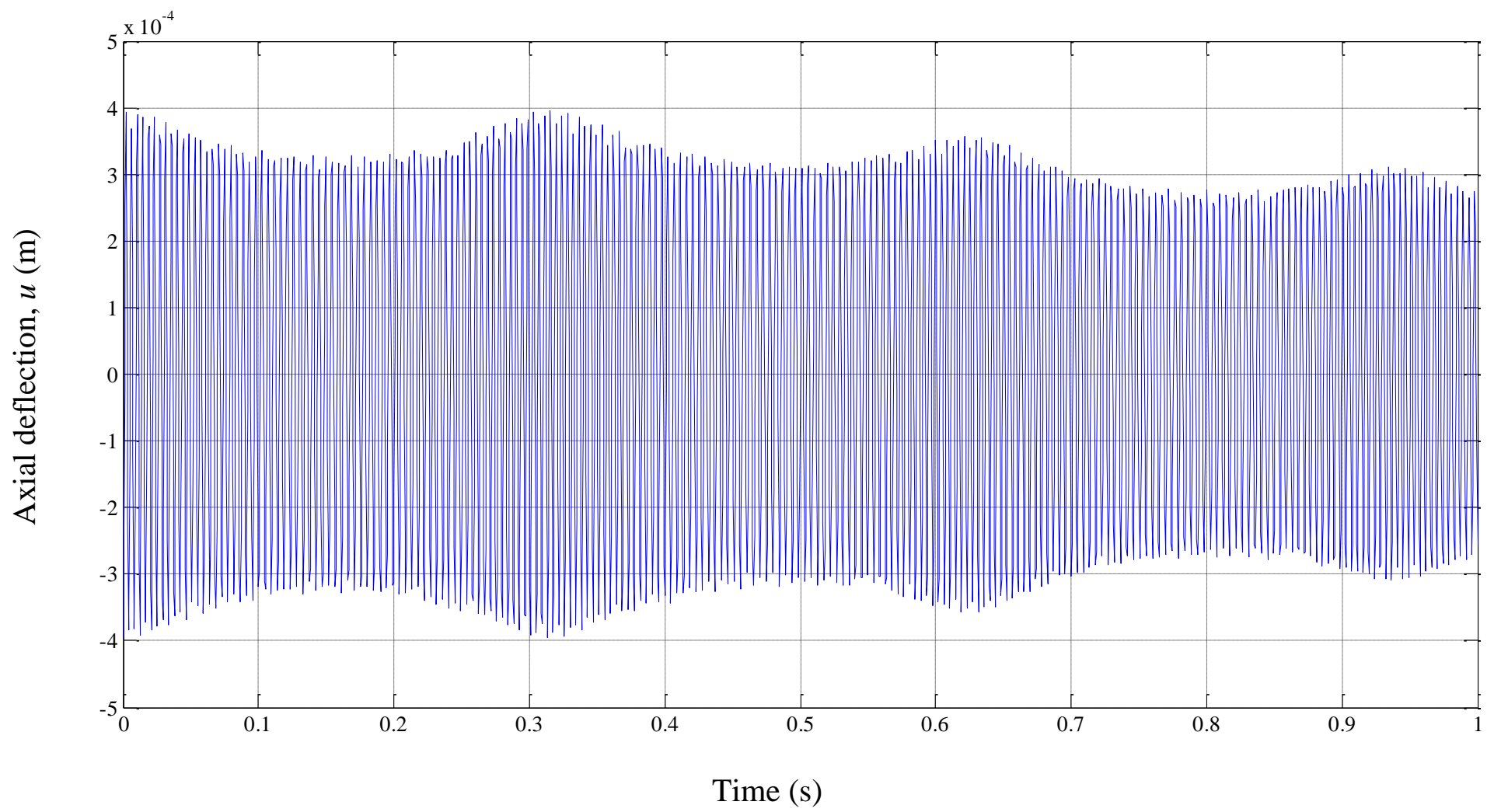

Figure 6.27 Axial deflection at the right end, $u, F S D T, \Omega=10 \mathrm{rad} / \mathrm{s},[0 / \pm 45]$ 
Table 6.12 Transverse tip deflection at the left end, $w$, FSDT, $\Omega=10 \mathrm{rad} / \mathrm{s},[0 / \pm 45]$

\begin{tabular}{|c|c|c|c|c|c|c|r|}
\hline$t(\mathrm{~s})$ & $w(\mathrm{~m})$ & $t(\mathrm{~s})$ & $w(\mathrm{~m})$ & $t(\mathrm{~s})$ & $w(\mathrm{~m})$ & $t(\mathrm{~s})$ & $w(\mathrm{~m})$ \\
\hline 0.000000 & 0.005000 & 0.258500 & 0.002590 & 0.503500 & 0.006722 & 0.750500 & 0.001557 \\
\hline 0.010500 & -0.004290 & 0.260500 & -0.002848 & 0.510500 & -0.006678 & 0.765000 & 0.001517 \\
\hline 0.021000 & 0.003979 & 0.273500 & -0.003232 & 0.524500 & -0.006588 & 0.772000 & -0.001521 \\
\hline 0.031500 & -0.003489 & 0.288500 & 0.003670 & 0.531500 & 0.006548 & 0.786000 & -0.001423 \\
\hline 0.046500 & 0.003047 & 0.299000 & -0.004232 & 0.543000 & -0.006455 & 0.793500 & 0.001484 \\
\hline 0.059500 & 0.002676 & 0.303000 & -0.004528 & 0.552000 & -0.006380 & 0.800500 & -0.001515 \\
\hline 0.068500 & 0.002484 & 0.317500 & 0.005169 & 0.561000 & -0.006302 & 0.814500 & -0.001476 \\
\hline 0.070500 & -0.002377 & 0.328000 & -0.005517 & 0.572000 & 0.006150 & 0.824000 & -0.001507 \\
\hline 0.086500 & 0.002194 & 0.336500 & -0.005940 & 0.583000 & -0.005951 & 0.838000 & -0.001637 \\
\hline 0.095500 & 0.002112 & 0.347000 & 0.006269 & 0.591500 & -0.005825 & 0.845000 & 0.001664 \\
\hline 0.102500 & -0.001969 & 0.353500 & -0.006267 & 0.600000 & -0.005513 & 0.854000 & 0.001696 \\
\hline 0.112000 & -0.001876 & 0.360000 & 0.006496 & 0.610500 & 0.005273 & 0.865500 & -0.001801 \\
\hline 0.126000 & -0.001859 & 0.373000 & 0.006675 & 0.621000 & -0.004896 & 0.874500 & -0.001957 \\
\hline 0.133000 & 0.001828 & 0.388500 & -0.006698 & 0.631500 & 0.004248 & 0.885500 & 0.002119 \\
\hline 0.140000 & -0.001776 & 0.393000 & -0.006777 & 0.640000 & 0.003852 & 0.896500 & -0.002347 \\
\hline 0.154500 & -0.001769 & 0.409000 & 0.006891 & 0.654500 & -0.003395 & 0.907000 & 0.002653 \\
\hline 0.161500 & 0.001807 & 0.416000 & -0.006914 & 0.663000 & -0.002839 & 0.918000 & -0.002823 \\
\hline 0.175500 & 0.001733 & 0.423000 & 0.006892 & 0.671500 & -0.002673 & 0.924000 & 0.003230 \\
\hline 0.183000 & -0.001810 & 0.430000 & -0.006878 & 0.680000 & -0.002407 & 0.936500 & 0.003718 \\
\hline 0.197000 & -0.001860 & 0.446500 & 0.006907 & 0.691000 & 0.002197 & 0.949000 & 0.004085 \\
\hline 0.206000 & -0.001892 & 0.453500 & -0.006840 & 0.700000 & 0.002045 & 0.959500 & -0.004407 \\
\hline 0.215500 & -0.001990 & 0.465500 & 0.006829 & 0.711500 & -0.001868 & 0.968000 & -0.004707 \\
\hline 0.222500 & 0.002145 & 0.475000 & 0.006839 & 0.720500 & -0.001802 & 0.978500 & 0.004785 \\
\hline 0.231500 & 0.002268 & 0.487000 & -0.006741 & 0.736500 & 0.001669 & 0.985000 & -0.004917 \\
\hline 0.249500 & 0.002582 & 0.494000 & 0.006746 & 0.743500 & -0.001631 & 0.998000 & -0.004990 \\
\hline
\end{tabular}




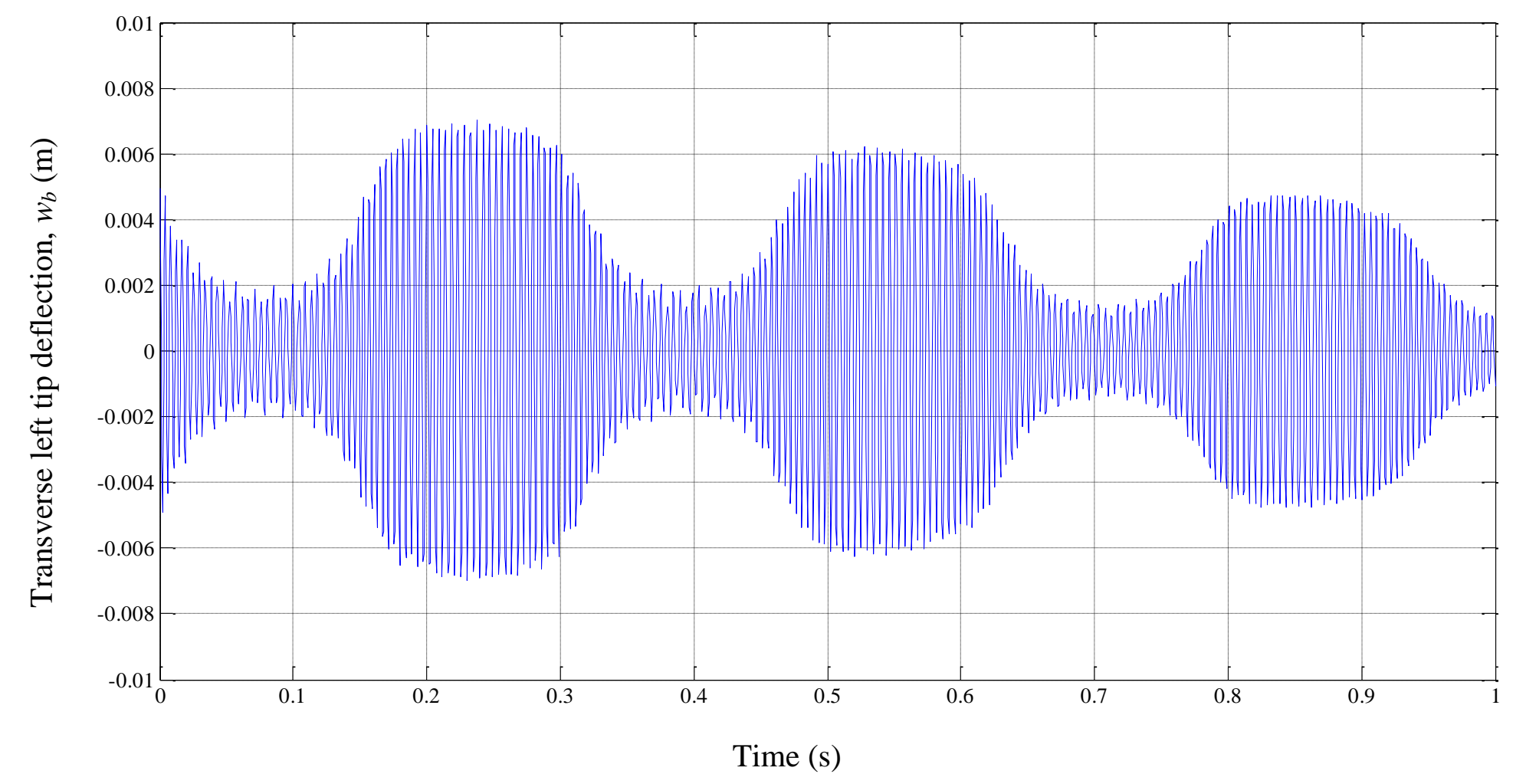

Figure 6.28 Transverse tip deflection at the left end, $w_{b}$, FSDT, $\Omega=20 \mathrm{rad} / \mathrm{s},[0 / \pm 45]$ 


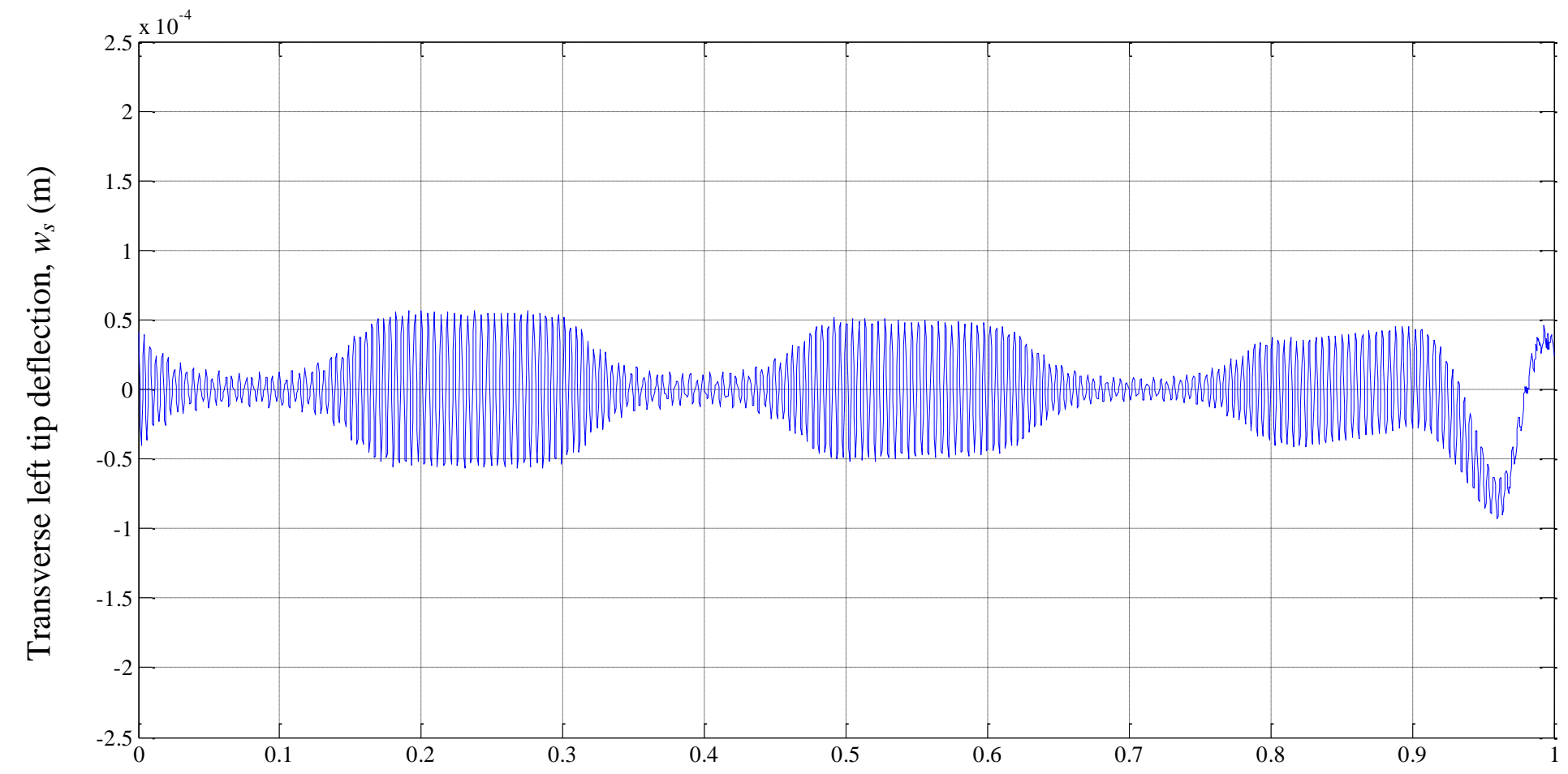

Time (s)

Figure 6.29 Transverse tip deflection at the left end, $w_{s}$, FSDT, $\Omega=20 \mathrm{rad} / \mathrm{s},[0 / \pm 45]$ 


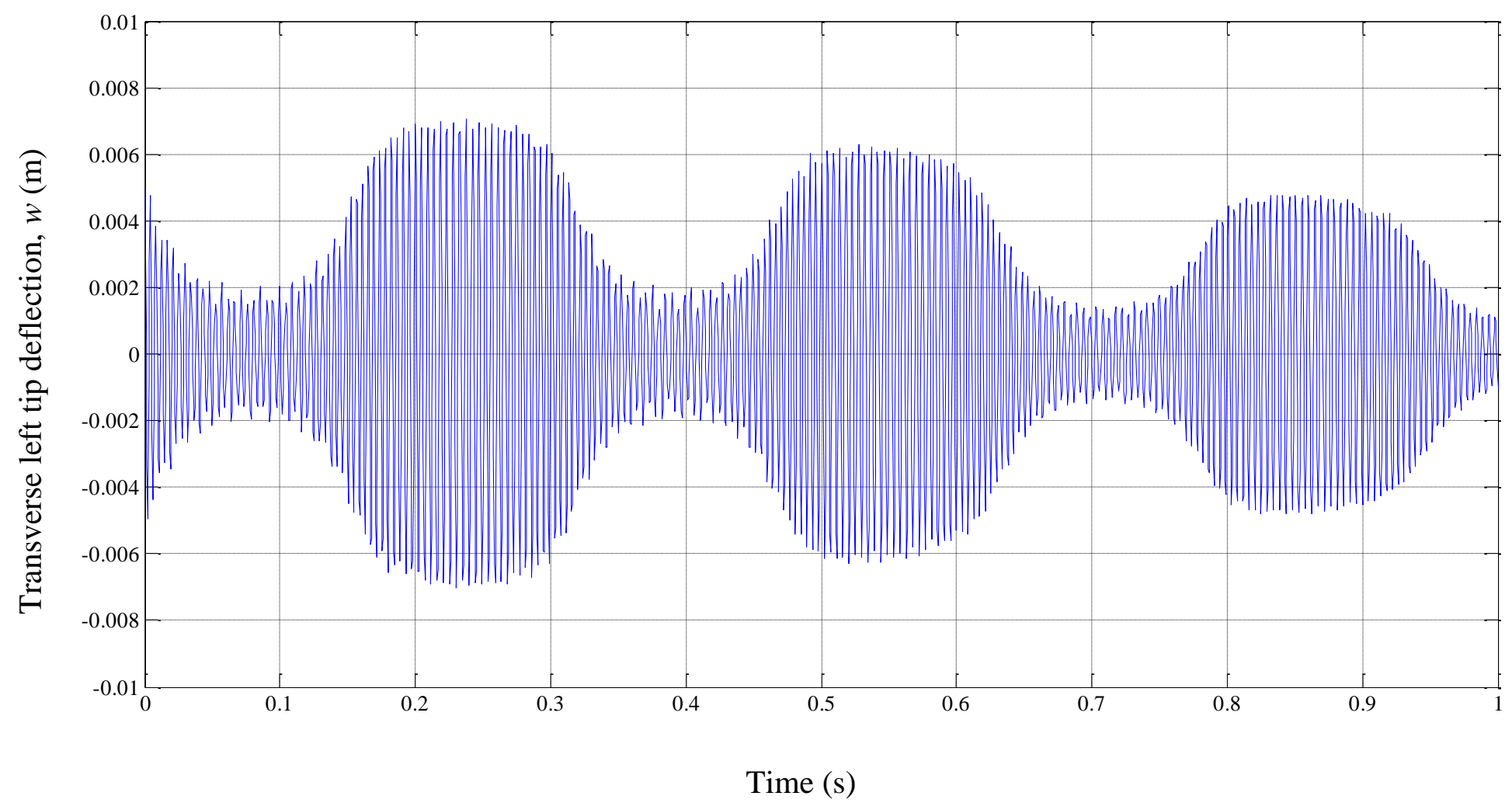

Figure 6.30 Transverse tip deflection at the left end, $w$, FSDT, $\Omega=20 \mathrm{rad} / \mathrm{s},[0 / \pm 45]$ 


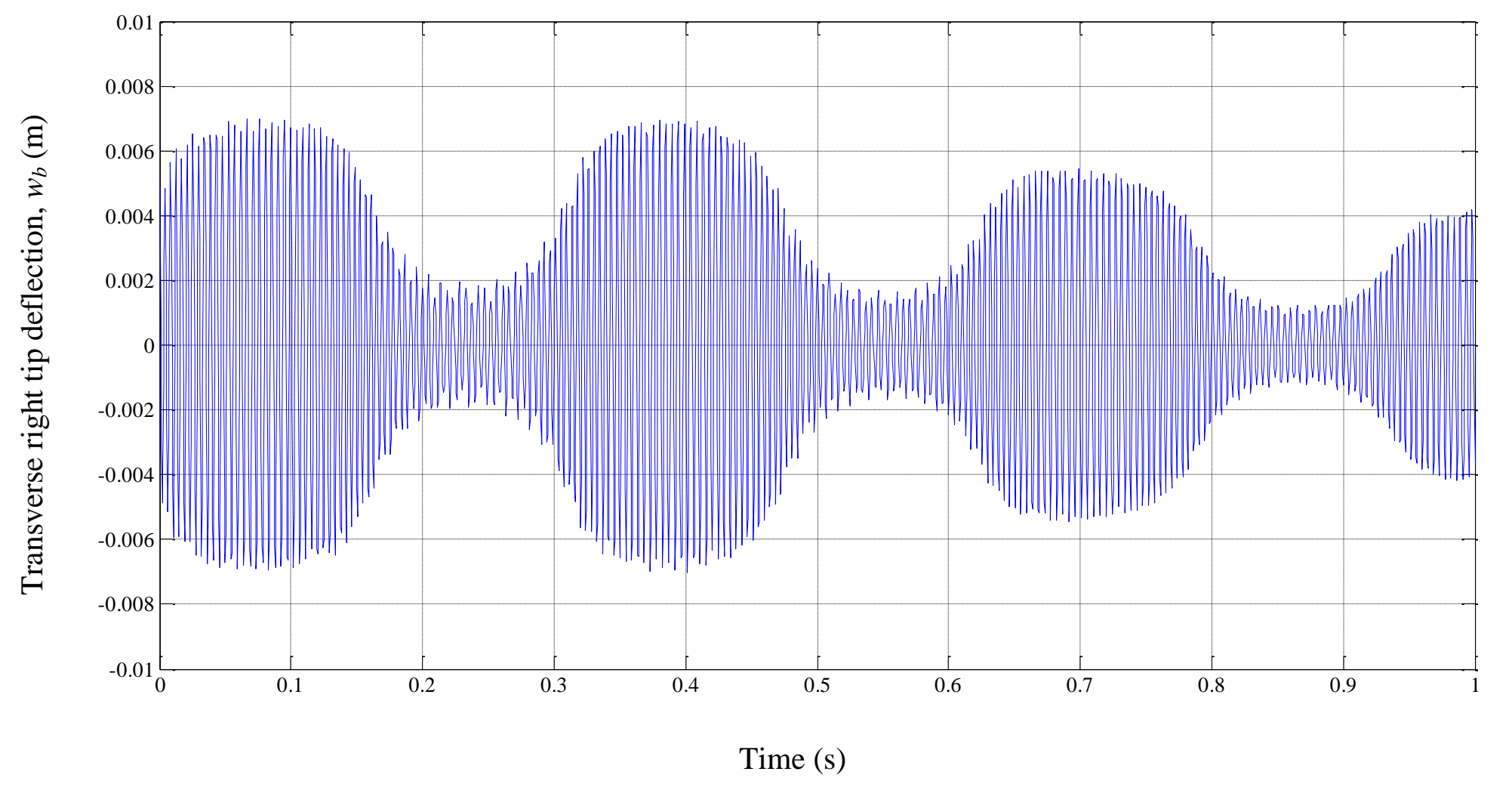

Figure 6.31 Transverse tip deflection at the right end, $w_{b}$, FSDT, $\Omega=20 \mathrm{rad} / \mathrm{s},[0 / \pm 45]$ 


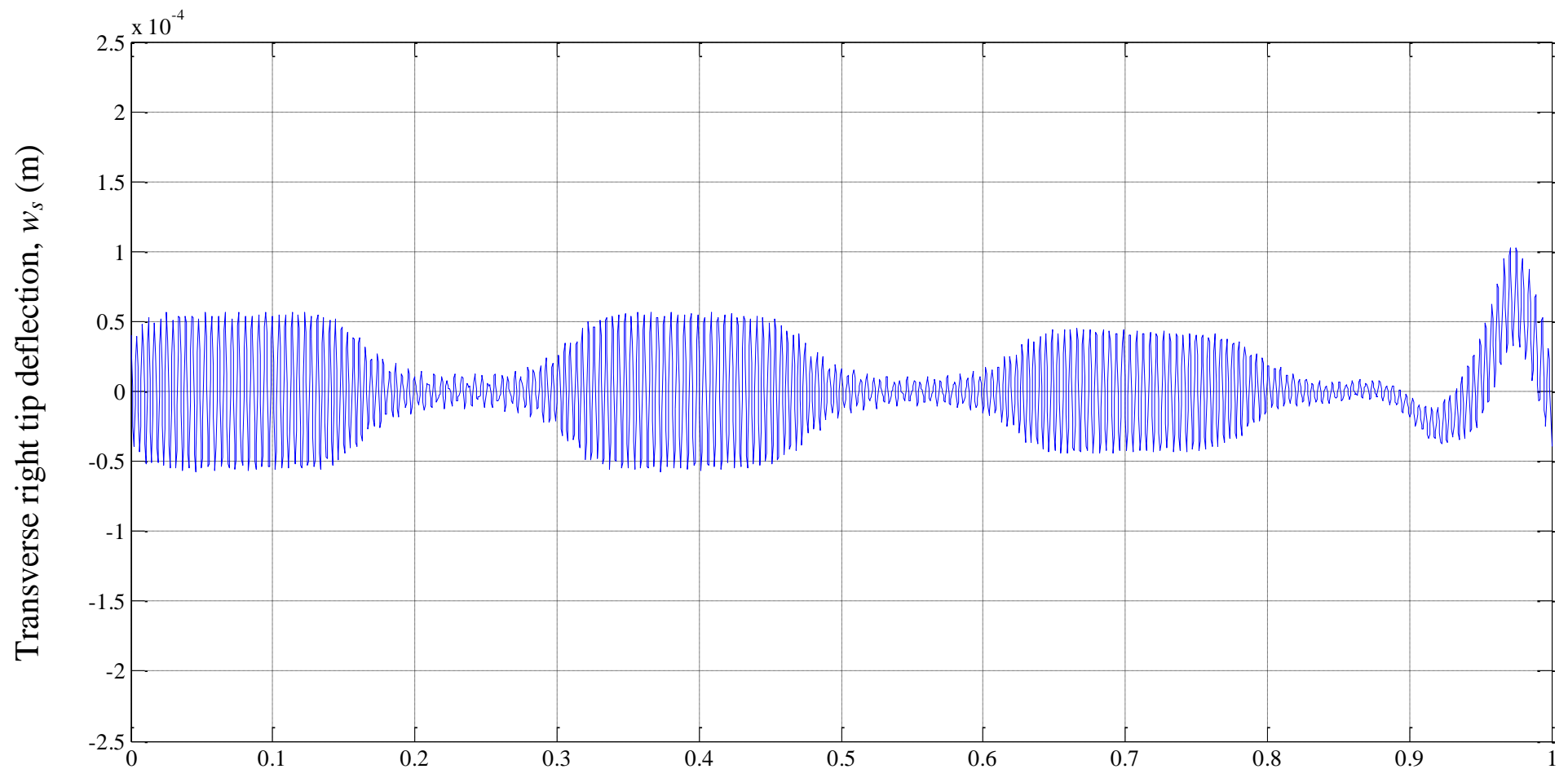

Time (s)

Figure 6.32 Transverse tip deflection at the right end, $w_{s}$, FSDT, $\Omega=20 \mathrm{rad} / \mathrm{s},[0 / \pm 45]$ 


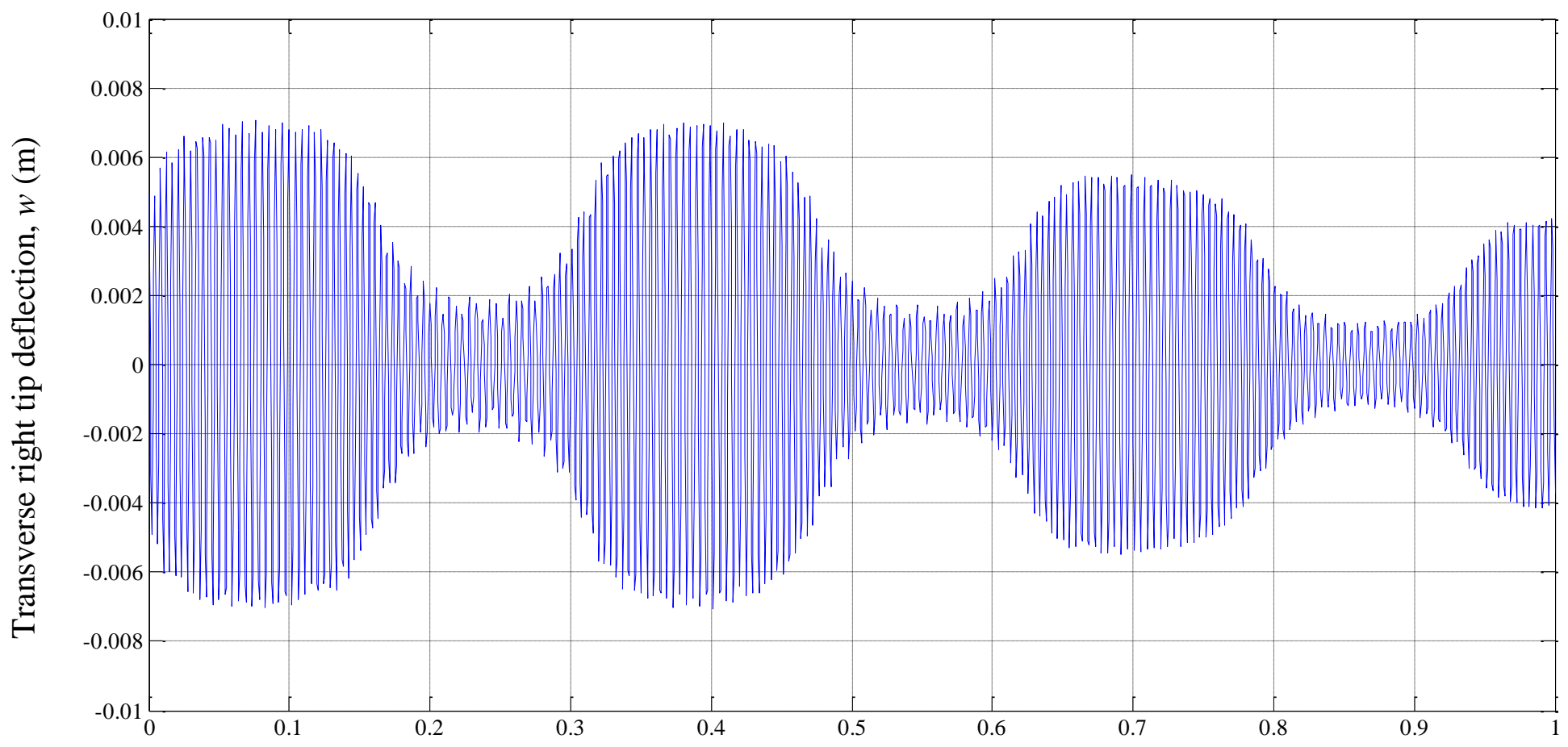

Time (s)

Figure 6.33 Transverse tip deflection at the right end, $w$, FSDT, $\Omega=20 \mathrm{rad} / \mathrm{s},[0 / \pm 45]$ 


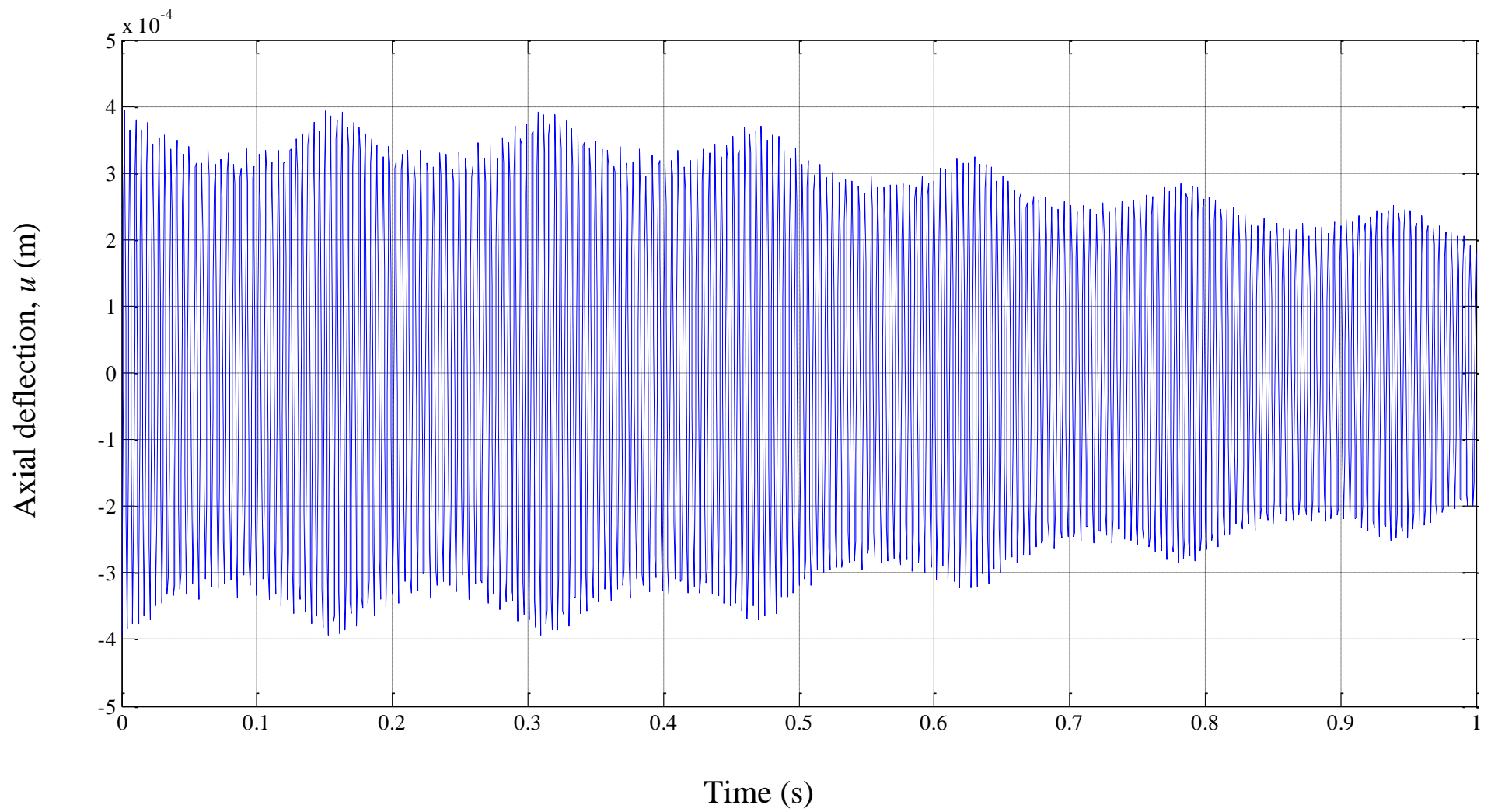

Figure 6.34 Axial deflection at the right end, $u$, FSDT, $\Omega=20 \mathrm{rad} / \mathrm{s},[0 / \pm 45]$ 
Table 6.13 Transverse tip deflection at the left end, $w$, FSDT, $\Omega=20 \mathrm{rad} / \mathrm{s},[0 / \pm 45]$

\begin{tabular}{|c|c|c|c|c|c|c|r|}
\hline$t(\mathrm{~s})$ & $w(\mathrm{~m})$ & $t(\mathrm{~s})$ & $w(\mathrm{~m})$ & $t(\mathrm{~s})$ & $w(\mathrm{~m})$ & $t(\mathrm{~s})$ & \multicolumn{1}{|c|}{$w(\mathrm{~m})$} \\
\hline 0.000000 & 0.005000 & 0.256500 & 0.006904 & 0.502500 & -0.006161 & 0.758500 & 0.002053 \\
\hline 0.010500 & -0.003586 & 0.268000 & -0.006903 & 0.513500 & 0.006167 & 0.769500 & -0.002613 \\
\hline 0.021000 & 0.003201 & 0.275000 & 0.006854 & 0.520500 & -0.006300 & 0.778000 & -0.002913 \\
\hline 0.030000 & 0.002712 & 0.286000 & -0.006701 & 0.534500 & -0.006240 & 0.788500 & 0.003819 \\
\hline 0.041000 & -0.002391 & 0.290500 & -0.006318 & 0.544000 & -0.006253 & 0.799000 & -0.004229 \\
\hline 0.050000 & -0.002149 & 0.301000 & 0.006029 & 0.556000 & 0.006193 & 0.809500 & 0.004548 \\
\hline 0.064000 & -0.002036 & 0.311500 & -0.005379 & 0.563000 & -0.006124 & 0.816000 & -0.004692 \\
\hline 0.078500 & -0.001963 & 0.320000 & -0.004079 & 0.572500 & -0.006064 & 0.825000 & -0.004803 \\
\hline 0.085500 & 0.002025 & 0.330500 & 0.003599 & 0.588500 & 0.005833 & 0.836500 & 0.004767 \\
\hline 0.092500 & -0.002050 & 0.341000 & -0.002805 & 0.597500 & 0.005730 & 0.843500 & -0.004784 \\
\hline 0.109000 & 0.002137 & 0.350000 & -0.002388 & 0.602000 & 0.005432 & 0.850500 & 0.004776 \\
\hline 0.118000 & 0.002360 & 0.361500 & 0.002193 & 0.610500 & 0.005305 & 0.862500 & -0.004793 \\
\hline 0.127000 & 0.002804 & 0.375500 & 0.002054 & 0.621000 & -0.004731 & 0.872000 & -0.004703 \\
\hline 0.138000 & -0.003368 & 0.382500 & -0.001951 & 0.631500 & 0.003652 & 0.881500 & -0.004670 \\
\hline 0.148500 & 0.004096 & 0.397000 & -0.001925 & 0.640000 & 0.003232 & 0.893000 & 0.004547 \\
\hline 0.159000 & -0.004823 & 0.404000 & 0.001996 & 0.650500 & -0.002496 & 0.900000 & -0.004542 \\
\hline 0.169500 & 0.005902 & 0.411000 & -0.002004 & 0.661500 & 0.002036 & 0.913500 & -0.004256 \\
\hline 0.178000 & 0.006190 & 0.429500 & -0.002164 & 0.670500 & 0.001734 & 0.920000 & 0.004222 \\
\hline 0.180000 & -0.006564 & 0.438500 & -0.002517 & 0.680000 & 0.001575 & 0.930500 & -0.003844 \\
\hline 0.191000 & 0.006802 & 0.449500 & 0.003002 & 0.696000 & -0.001494 & 0.941000 & 0.003123 \\
\hline 0.200000 & 0.006914 & 0.458000 & 0.003465 & 0.703000 & 0.001424 & 0.951500 & -0.002643 \\
\hline 0.218500 & 0.006984 & 0.468500 & -0.004149 & 0.717500 & 0.001438 & 0.960000 & -0.002178 \\
\hline 0.228000 & 0.006942 & 0.479000 & 0.005262 & 0.724500 & -0.001501 & 0.973000 & -0.001682 \\
\hline 0.237500 & 0.007081 & 0.489500 & -0.005801 & 0.738500 & -0.001611 & 0.982000 & -0.001374 \\
\hline 0.247000 & 0.006966 & 0.491500 & 0.006016 & 0.747500 & -0.001752 & 0.991000 & -0.001204 \\
\hline
\end{tabular}




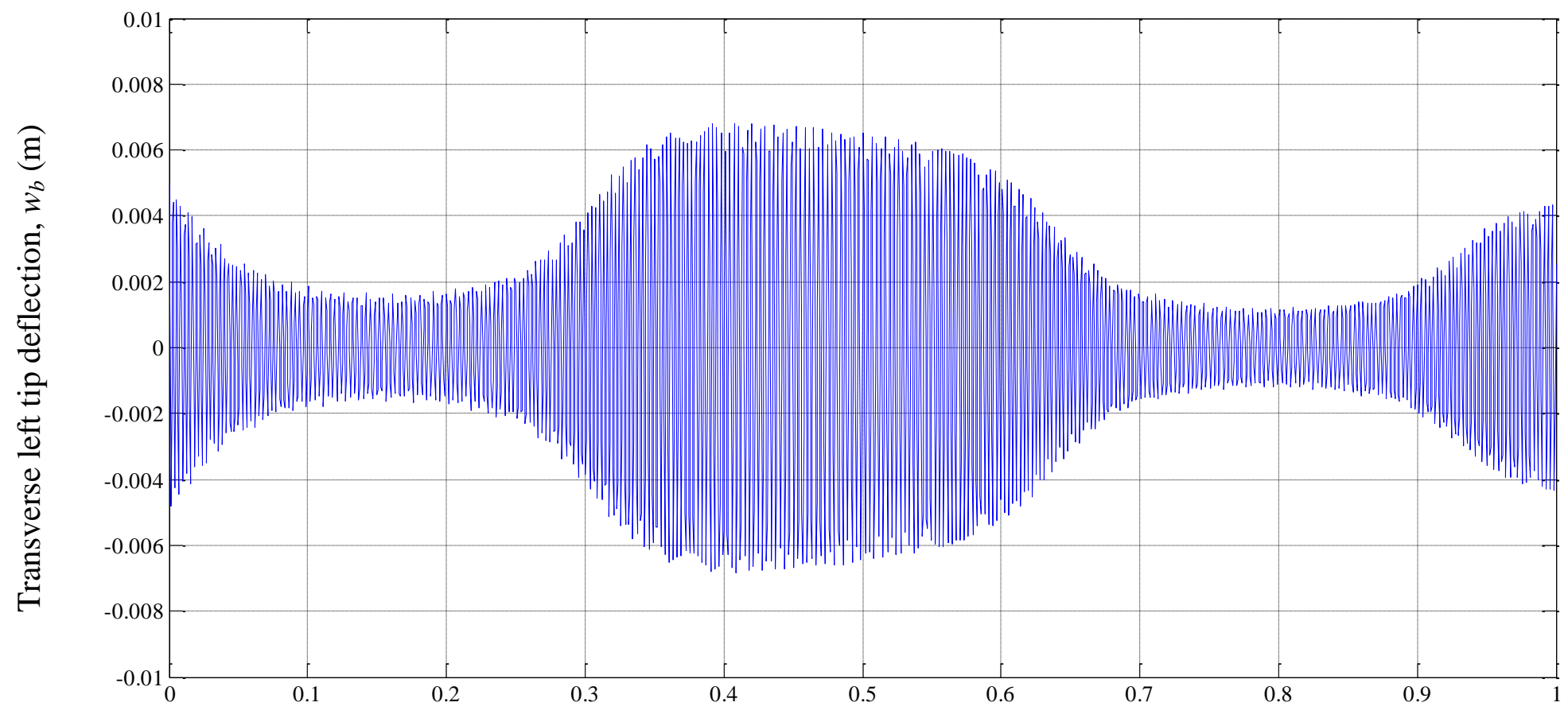

Time (s)

Figure 6.35 Transverse tip deflection at the left end, $w_{b}$, FSDT, $\Omega=10 \mathrm{rad} / \mathrm{s},[0 / \pm 20]$ 


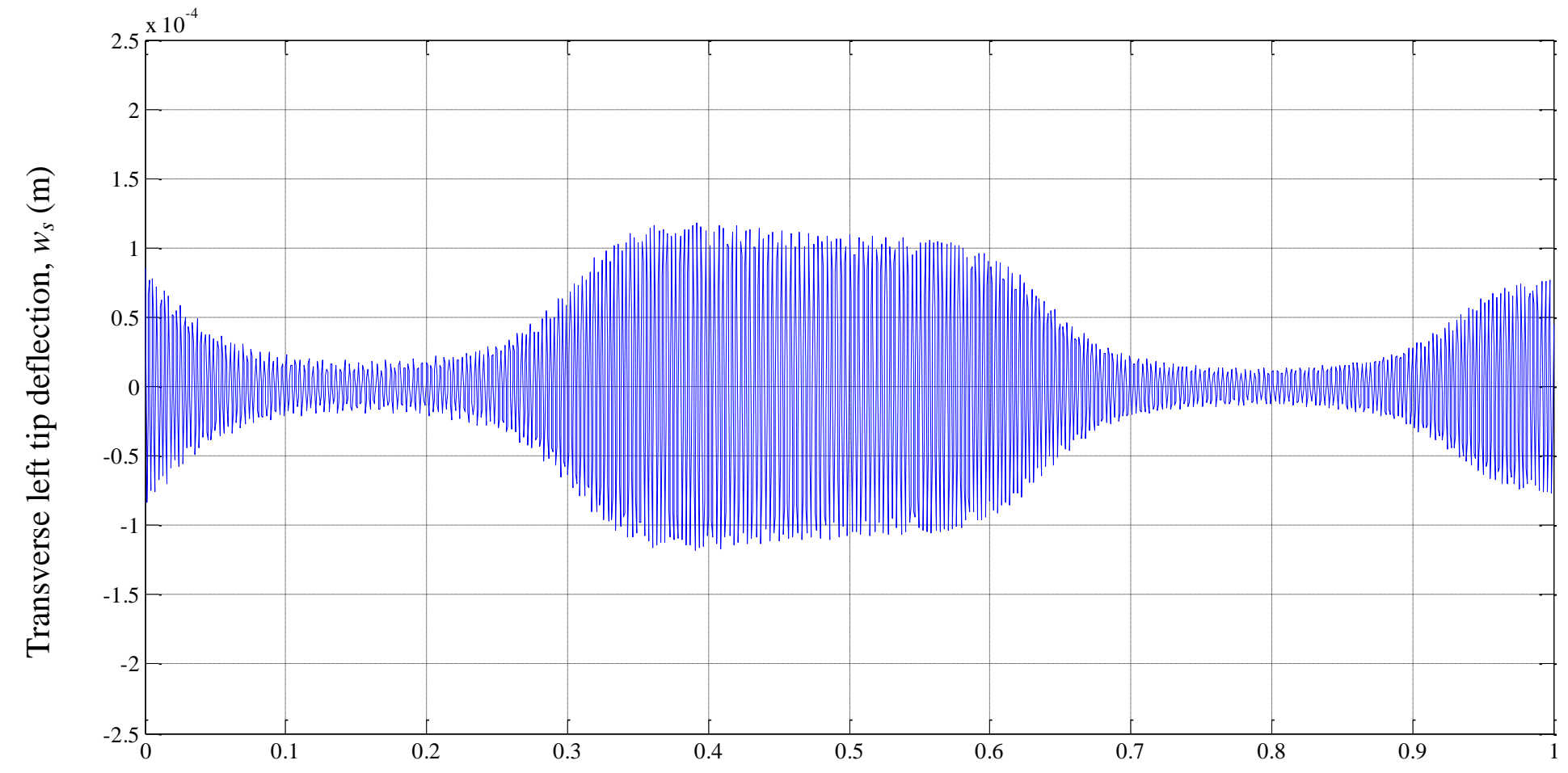

Time (s)

Figure 6.36 Transverse tip deflection at the left end, $w_{s}$, FSDT, $\Omega=10 \mathrm{rad} / \mathrm{s},[0 / \pm 20]$ 


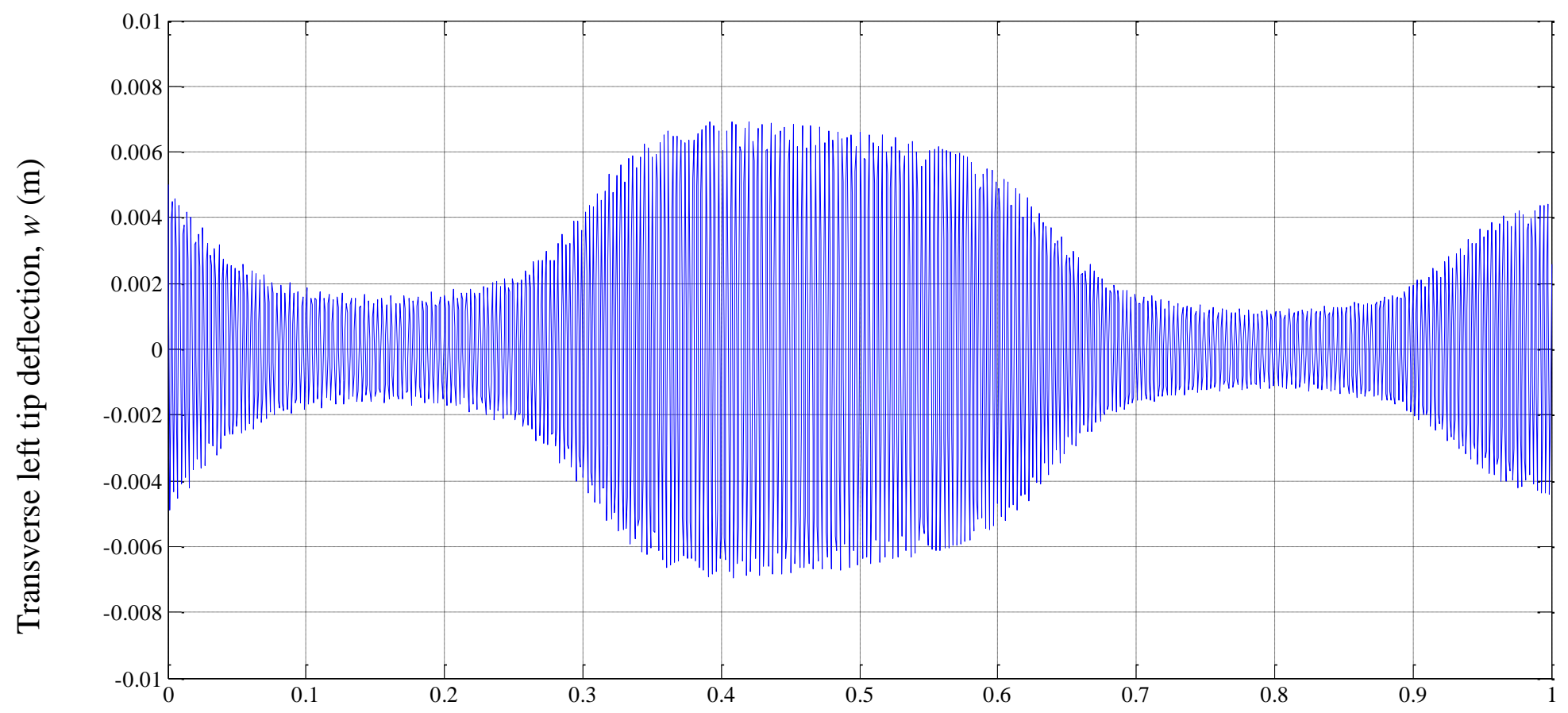

Time (s)

Figure 6.37 Transverse tip deflection at the left end, $w$, FSDT, $\Omega=10 \mathrm{rad} / \mathrm{s},[0 / \pm 20]$ 


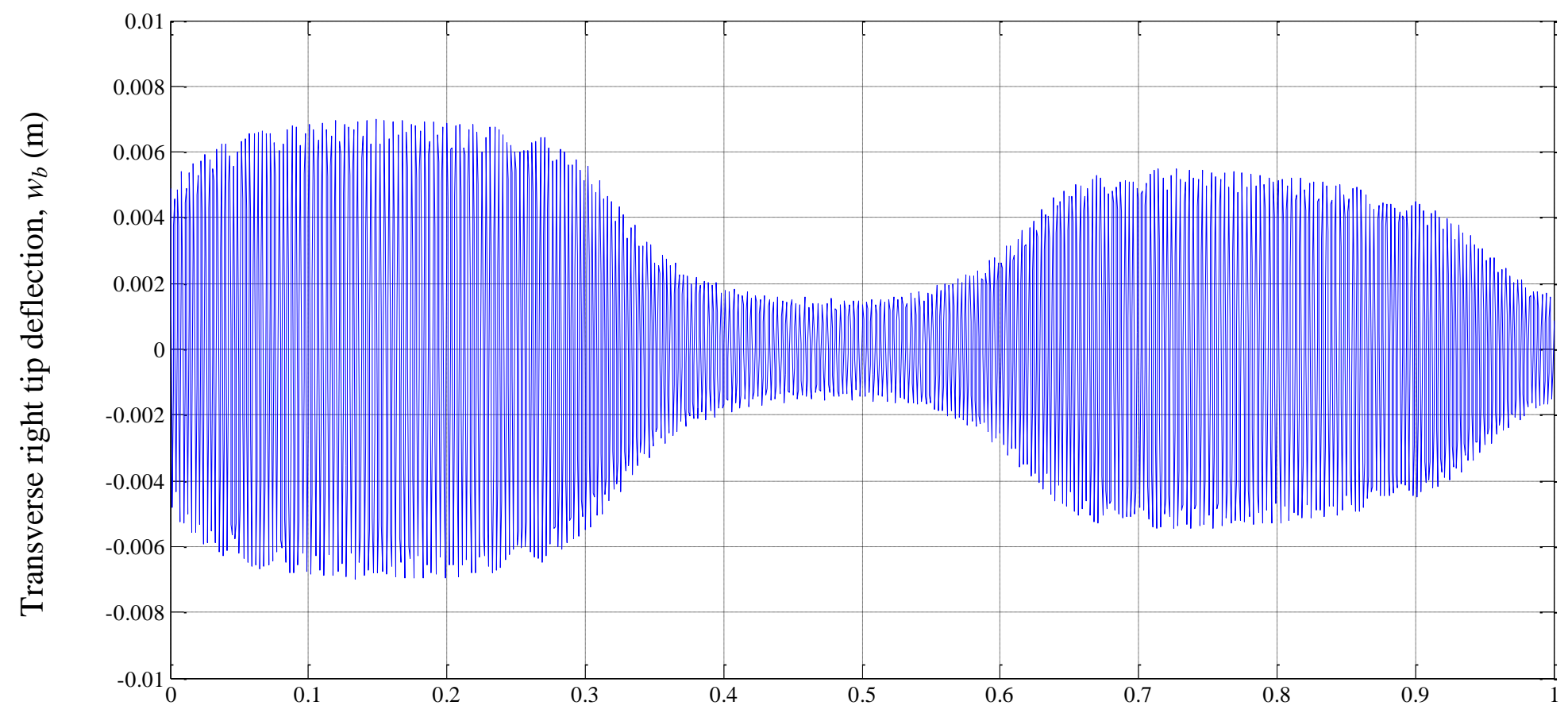

Time (s)

Figure 6.38 Transverse tip deflection at the right end, $w_{b}$, FSDT, $\Omega=10 \mathrm{rad} / \mathrm{s},[0 / \pm 20]$ 


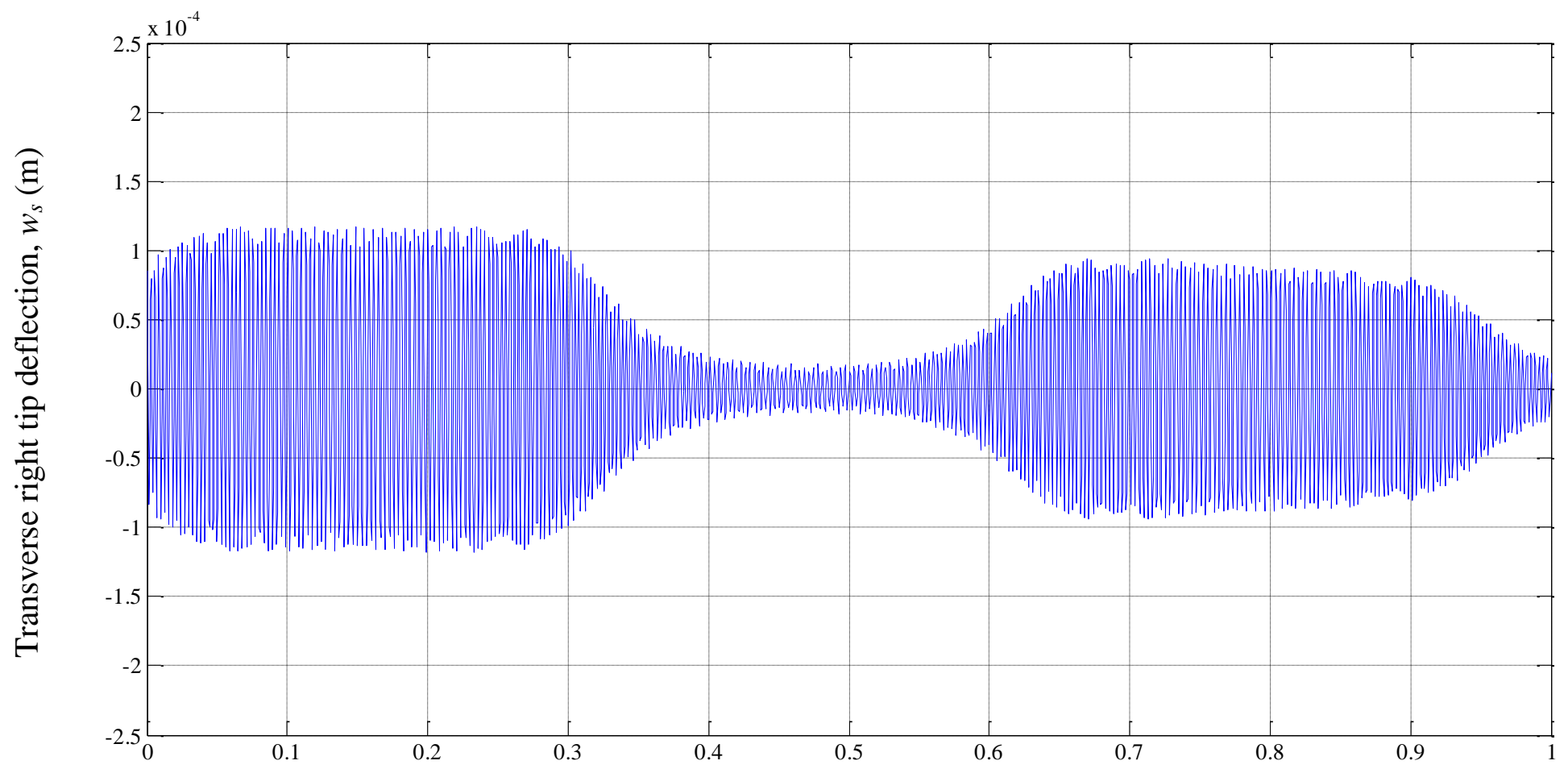

Time (s)

Figure 6.39 Transverse tip deflection at the right end, $w_{s}$, FSDT, $\Omega=10 \mathrm{rad} / \mathrm{s},[0 / \pm 20]$ 


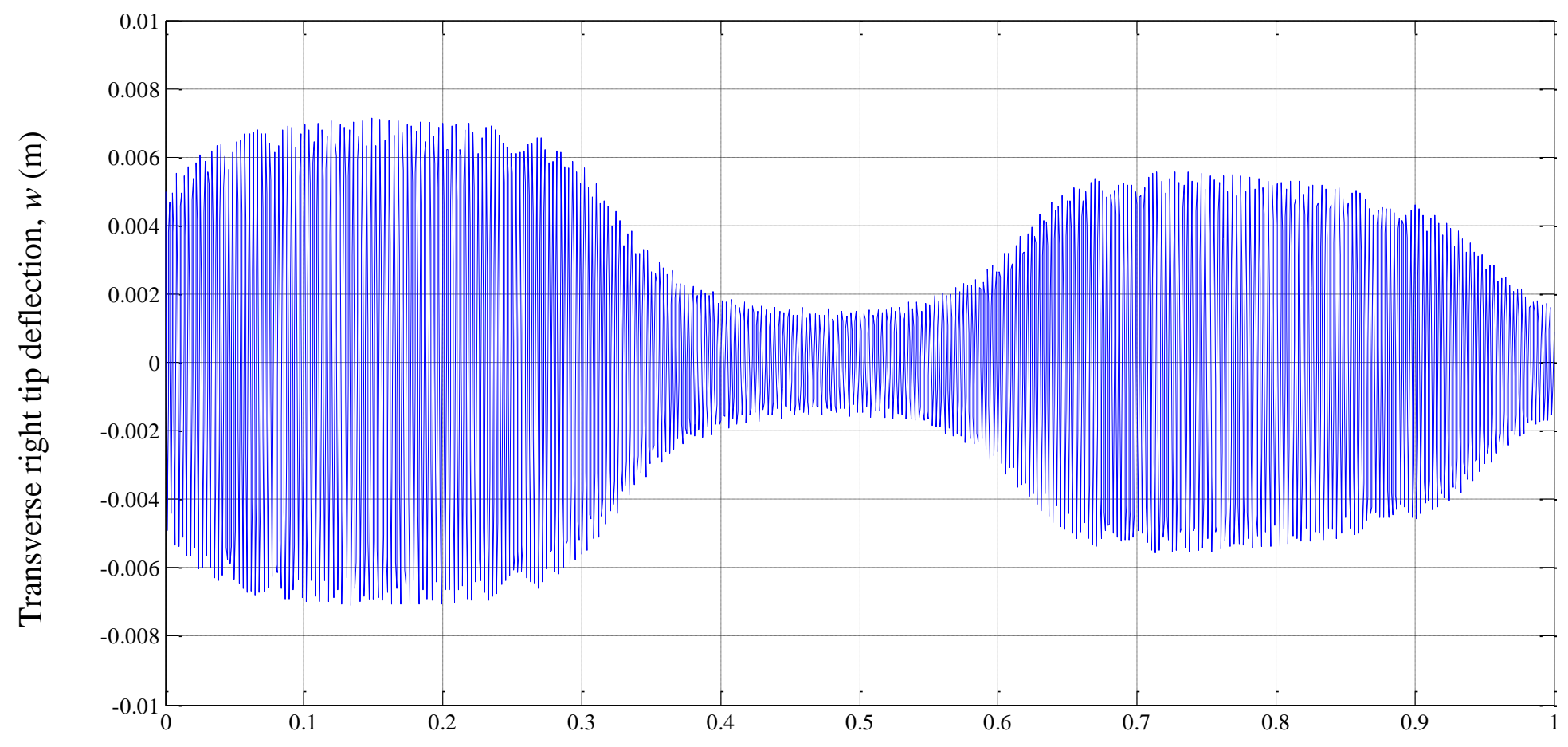

Time (s)

Figure 6.40 Transverse tip deflection at the right end, $w$, FSDT, $\Omega=10 \mathrm{rad} / \mathrm{s},[0 / \pm 20]$ 


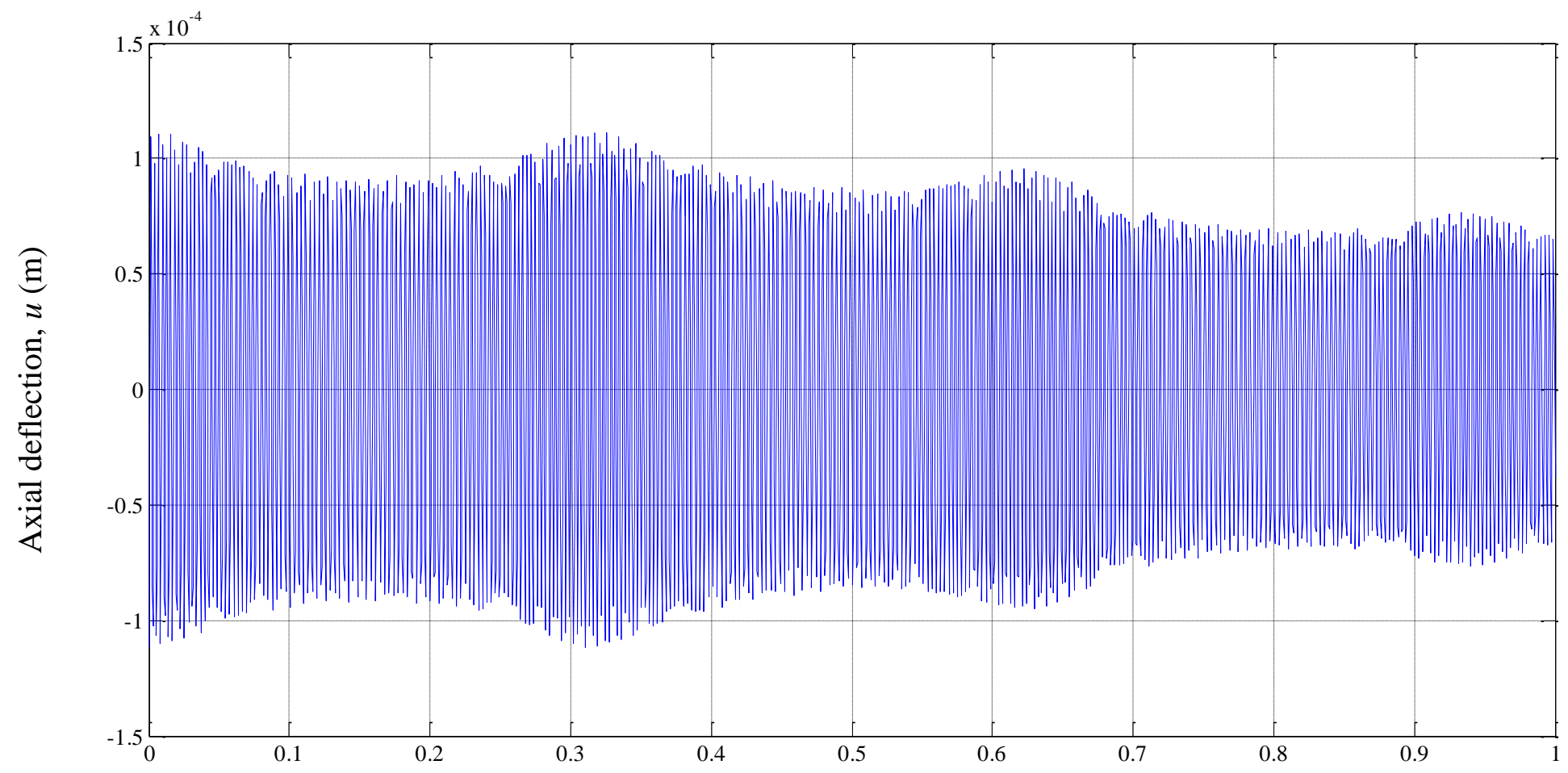

Time (s)

Figure 6.41 Axial deflection at the right end, $u$, FSDT, $\Omega=10 \mathrm{rad} / \mathrm{s},[0 / \pm 20]$ 
Table 6.14 Transverse tip deflection at the left end, $w$, FSDT, $\Omega=10 \mathrm{rad} / \mathrm{s},[0 / \pm 20]$

\begin{tabular}{|c|c|c|c|c|c|c|c|}
\hline$t(\mathrm{~s})$ & $w(\mathrm{~m})$ & $t(\mathrm{~s})$ & $w(\mathrm{~m})$ & $t(\mathrm{~s})$ & $w(\mathrm{~m})$ & $t(\mathrm{~s})$ & $w(\mathrm{~m})$ \\
\hline 0.000000 & 0.005000 & 0.258500 & 0.002383 & 0.500500 & 0.006616 & 0.755500 & 0.001254 \\
\hline 0.015500 & -0.004225 & 0.266000 & -0.002765 & 0.516500 & 0.006512 & 0.760500 & -0.001280 \\
\hline 0.025500 & 0.003679 & 0.273500 & 0.002989 & 0.526000 & 0.006443 & 0.775000 & 0.001225 \\
\hline 0.035500 & -0.003203 & 0.285000 & 0.003483 & 0.537000 & -0.006342 & 0.781500 & 0.001195 \\
\hline 0.040000 & 0.002756 & 0.295000 & -0.004008 & 0.540000 & -0.006193 & 0.796000 & -0.001208 \\
\hline 0.054500 & 0.002595 & 0.309000 & -0.004644 & 0.557000 & 0.006167 & 0.809000 & -0.001198 \\
\hline 0.062000 & -0.002444 & 0.319000 & 0.005346 & 0.560000 & 0.006073 & 0.810500 & 0.001235 \\
\hline 0.071000 & -0.002090 & 0.327500 & 0.005591 & 0.572000 & 0.005976 & 0.825000 & -0.001279 \\
\hline 0.088000 & 0.002013 & 0.334500 & -0.005920 & 0.581000 & 0.005691 & 0.836000 & 0.001298 \\
\hline 0.099000 & -0.001813 & 0.344500 & 0.006253 & 0.592500 & 0.005500 & 0.847000 & -0.001344 \\
\hline 0.100500 & 0.001881 & 0.359000 & 0.006527 & 0.605500 & -0.005190 & 0.859500 & -0.001471 \\
\hline 0.111500 & -0.001795 & 0.360500 & -0.006622 & 0.612500 & 0.004895 & 0.865500 & -0.001423 \\
\hline 0.126000 & 0.001690 & 0.377000 & 0.006379 & 0.621000 & 0.004628 & 0.879500 & 0.001592 \\
\hline 0.137000 & -0.001598 & 0.389000 & 0.006811 & 0.631000 & -0.004096 & 0.887000 & -0.001660 \\
\hline 0.142000 & 0.001671 & 0.392000 & 0.006905 & 0.645000 & -0.003445 & 0.898500 & -0.001912 \\
\hline 0.156500 & -0.001646 & 0.409000 & -0.006954 & 0.655000 & 0.002967 & 0.904500 & -0.002151 \\
\hline 0.161500 & 0.001641 & 0.418500 & -0.006881 & 0.666500 & 0.002566 & 0.916000 & -0.002467 \\
\hline 0.176000 & -0.001691 & 0.420000 & 0.006929 & 0.671000 & -0.002244 & 0.926000 & 0.002804 \\
\hline 0.185500 & -0.001614 & 0.436000 & 0.006889 & 0.684000 & 0.001948 & 0.938500 & -0.003172 \\
\hline 0.190500 & 0.001741 & 0.444000 & -0.006833 & 0.691500 & -0.001841 & 0.948500 & 0.003662 \\
\hline 0.206500 & 0.001821 & 0.452000 & 0.006842 & 0.705500 & 0.001587 & 0.957000 & 0.003849 \\
\hline 0.217500 & -0.001923 & 0.465000 & 0.006789 & 0.711500 & 0.001635 & 0.965500 & 0.004058 \\
\hline 0.228500 & 0.001917 & 0.471500 & 0.006749 & 0.724000 & 0.001467 & 0.977000 & 0.004220 \\
\hline 0.236000 & -0.002151 & 0.486000 & -0.006702 & 0.735000 & -0.001396 & 0.988500 & 0.004210 \\
\hline 0.249500 & 0.002151 & 0.492500 & -0.006625 & 0.746000 & 0.001356 & 0.999000 & -0.004417 \\
\hline
\end{tabular}




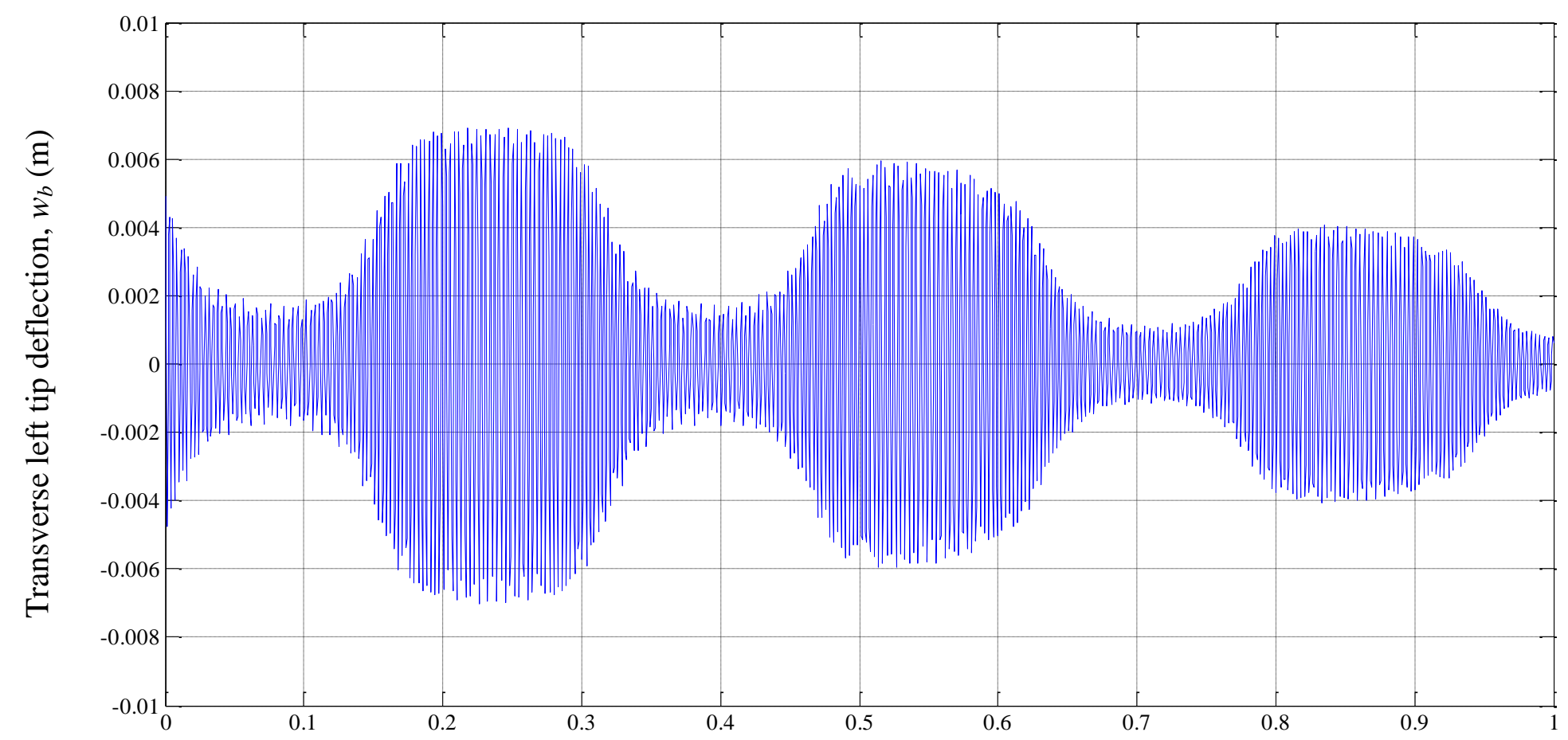

Time (s)

Figure 6.42 Transverse tip deflection at the left end, $w_{b}$, FSDT, $\Omega=20 \mathrm{rad} / \mathrm{s},[0 / \pm 20]$ 


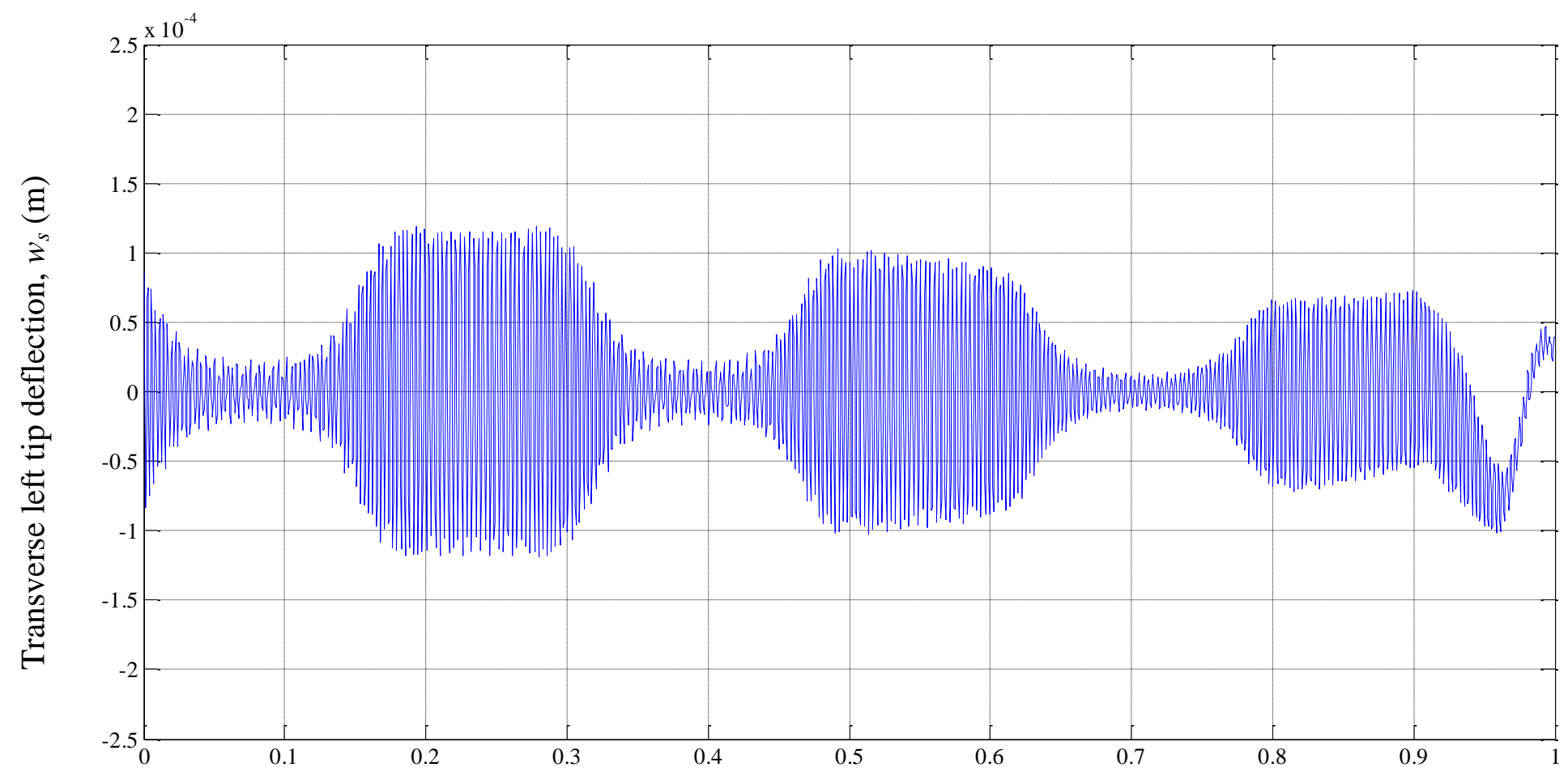

Time (s)

Figure 6.43 Transverse tip deflection at the left end, $w_{s}$, FSDT, $\Omega=20 \mathrm{rad} / \mathrm{s},[0 / \pm 20]$ 


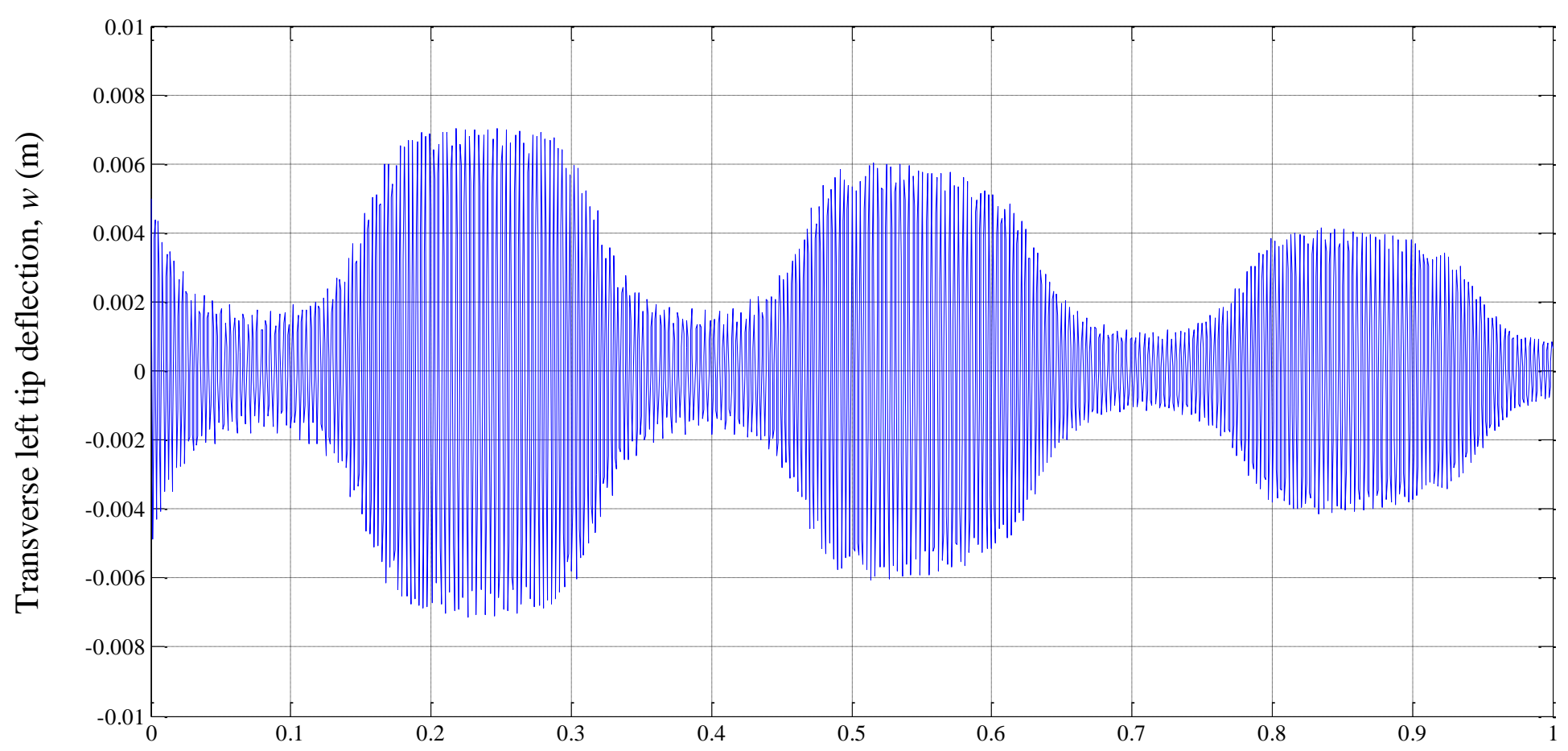

Time (s)

Figure 6.44 Transverse tip deflection at the left end, $w$, FSDT, $\Omega=20 \mathrm{rad} / \mathrm{s},[0 / \pm 20]$ 


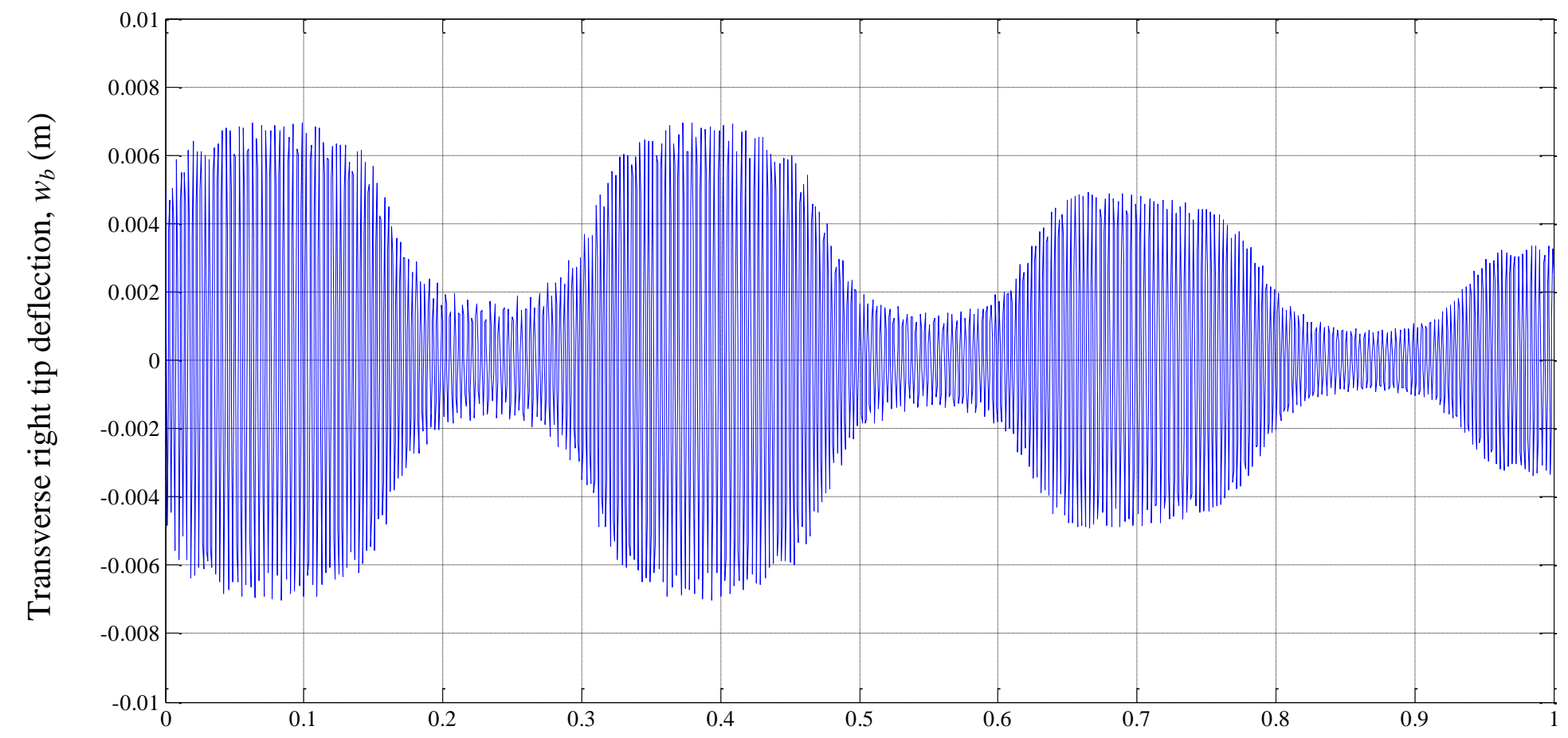

Time (s)

Figure 6.45 Transverse tip deflection at the right end, $w_{b}$, FSDT, $\Omega=20 \mathrm{rad} / \mathrm{s},[0 / \pm 20]$ 


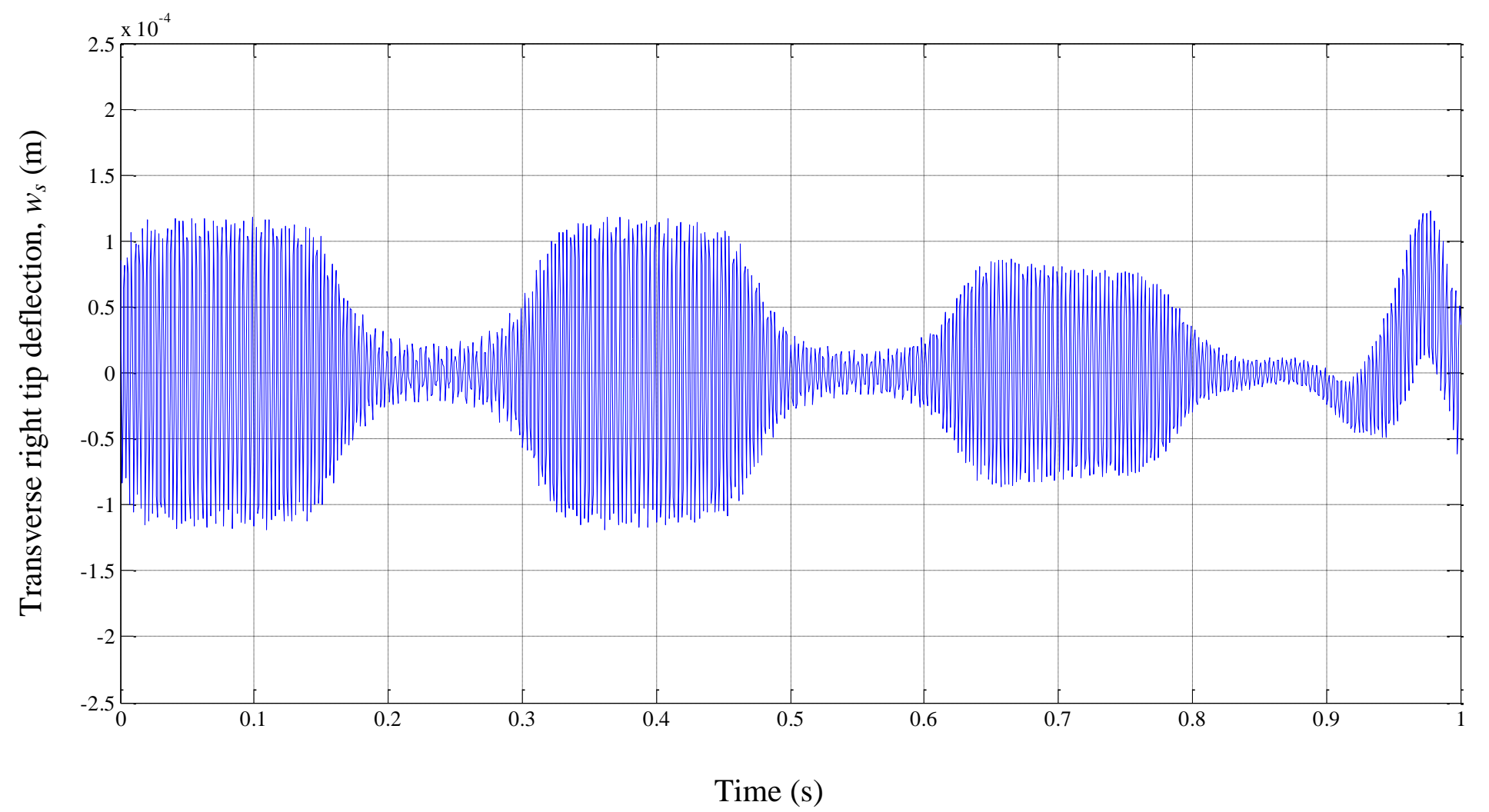

Figure 6.46 Transverse tip deflection at the right end, $w_{s}$, FSDT, $\Omega=20 \mathrm{rad} / \mathrm{s},[0 / \pm 20]$ 


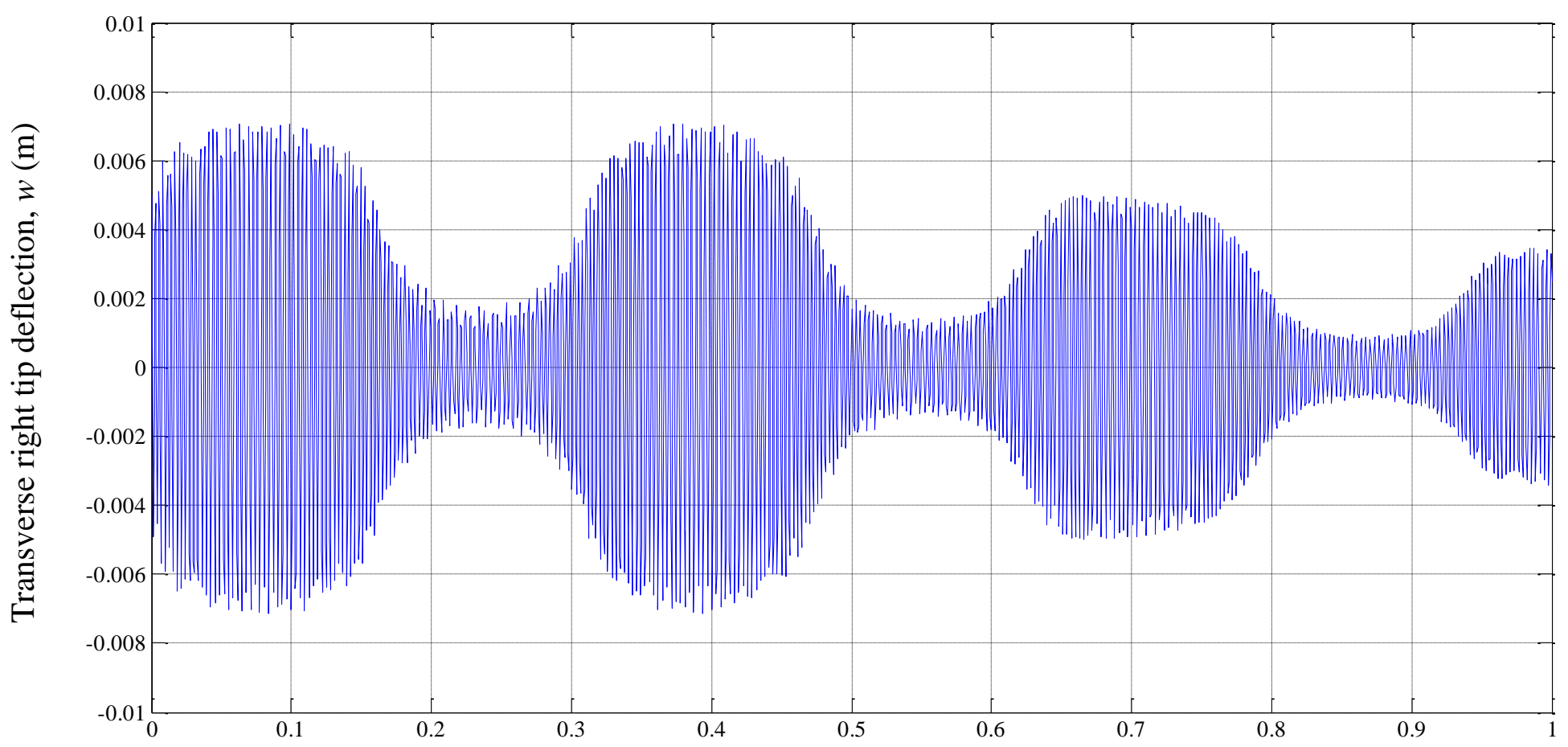

Time (s)

Figure 6.47 Transverse tip deflection at the right end, $w$, FSDT, $\Omega=20 \mathrm{rad} / \mathrm{s},[0 / \pm 20]$ 


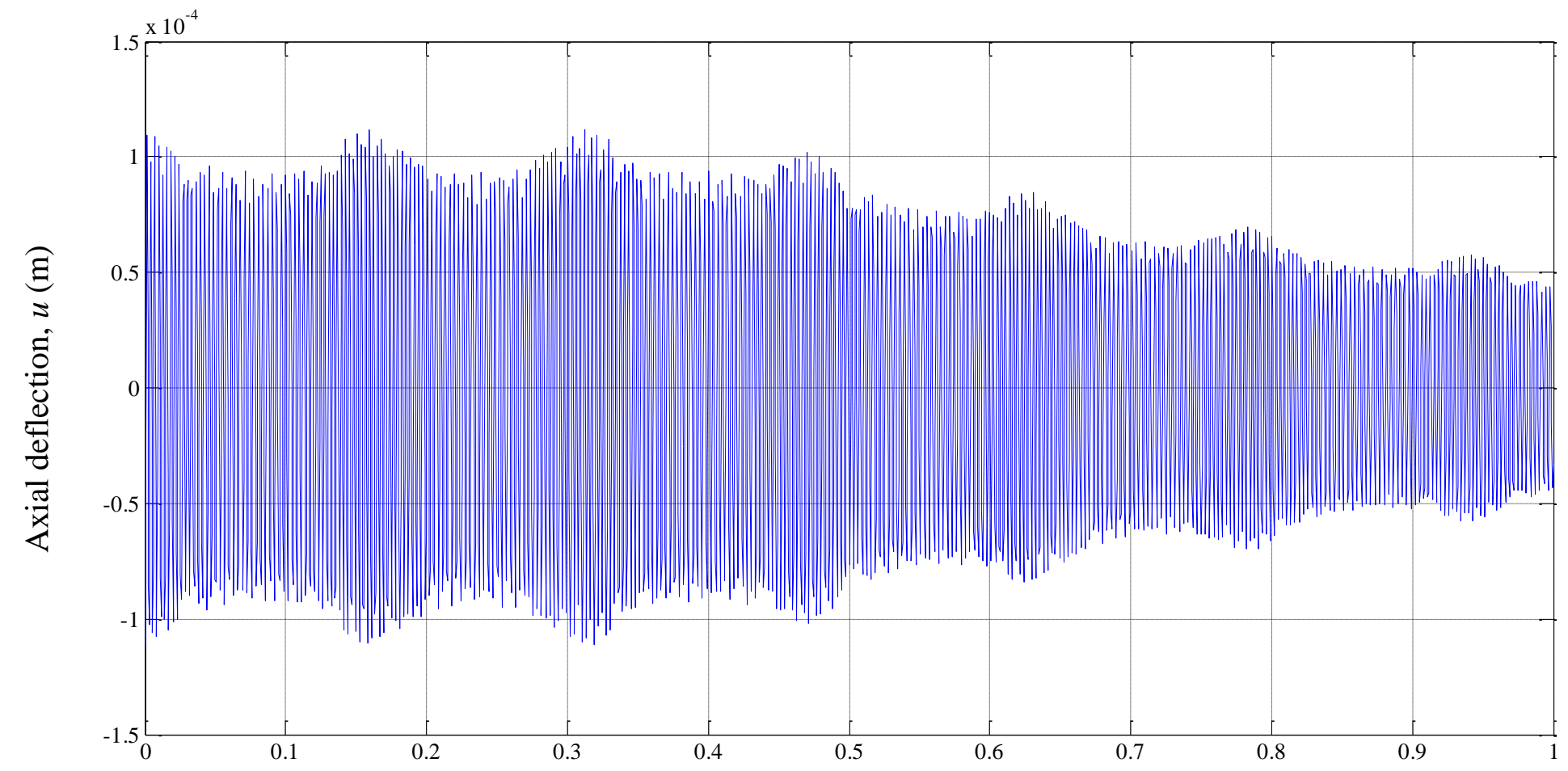

Time (s)

Figure 6.48 Axial deflection at the right end, $u, F S D T, \Omega=20 \mathrm{rad} / \mathrm{s},[0 / \pm 20]$ 
Table 6.15 Transverse tip deflection at the left end, $w$, FSDT, $\Omega=20 \mathrm{rad} / \mathrm{s},[0 / \pm 20]$

\begin{tabular}{|c|c|c|c|c|c|c|c|}
\hline$t(\mathrm{~s})$ & $w(\mathrm{~m})$ & $t(\mathrm{~s})$ & $w(\mathrm{~m})$ & $t(\mathrm{~s})$ & $w(\mathrm{~m})$ & $t(\mathrm{~s})$ & $w(\mathrm{~m})$ \\
\hline 0.000000 & 0.005000 & 0.253500 & 0.006999 & 0.509500 & 0.005637 & 0.756000 & 0.001621 \\
\hline 0.010000 & -0.003498 & 0.261500 & -0.007044 & 0.514000 & -0.006054 & 0.763500 & -0.001875 \\
\hline 0.023000 & 0.002872 & 0.278500 & 0.006892 & 0.526500 & -0.006044 & 0.775000 & -0.002440 \\
\hline 0.030500 & -0.002293 & 0.280000 & -0.006873 & 0.534500 & 0.006005 & 0.789000 & -0.003278 \\
\hline 0.045500 & -0.002099 & 0.290500 & 0.006461 & 0.541000 & 0.005953 & 0.799000 & 0.003822 \\
\hline 0.056500 & 0.001930 & 0.303500 & -0.006022 & 0.555500 & -0.005918 & 0.800500 & -0.003818 \\
\hline 0.061500 & -0.001814 & 0.310500 & 0.005226 & 0.562000 & -0.005782 & 0.815000 & -0.004007 \\
\hline 0.071000 & -0.001799 & 0.320500 & -0.004230 & 0.570000 & 0.005774 & 0.821000 & -0.003963 \\
\hline 0.085500 & 0.001728 & 0.330500 & 0.003351 & 0.581000 & -0.005626 & 0.833500 & -0.004143 \\
\hline 0.090500 & -0.001821 & 0.340500 & -0.002582 & 0.593500 & -0.005244 & 0.844500 & 0.004102 \\
\hline 0.106500 & -0.001985 & 0.354000 & 0.002084 & 0.601000 & 0.005097 & 0.851000 & 0.004112 \\
\hline 0.119000 & -0.002094 & 0.365000 & -0.001943 & 0.612500 & 0.004841 & 0.865500 & -0.004049 \\
\hline 0.125000 & -0.002452 & 0.370000 & 0.001847 & 0.622500 & -0.004342 & 0.872000 & -0.003996 \\
\hline 0.139500 & -0.002801 & 0.381000 & -0.001843 & 0.631000 & -0.003588 & 0.880000 & 0.003953 \\
\hline 0.144000 & 0.003697 & 0.395500 & 0.001756 & 0.641000 & 0.002571 & 0.897500 & -0.003796 \\
\hline 0.159500 & -0.005108 & 0.400500 & -0.001837 & 0.652500 & 0.002048 & 0.900500 & -0.003741 \\
\hline 0.168000 & -0.006129 & 0.416500 & -0.001842 & 0.661500 & 0.001730 & 0.918500 & -0.003348 \\
\hline 0.178000 & 0.006534 & 0.427500 & 0.002072 & 0.670500 & 0.001274 & 0.924500 & -0.003421 \\
\hline 0.185500 & -0.006744 & 0.433500 & 0.002145 & 0.680000 & 0.001352 & 0.933000 & -0.003071 \\
\hline 0.193000 & 0.006900 & 0.448500 & 0.002763 & 0.691000 & -0.001196 & 0.941500 & -0.002646 \\
\hline 0.208500 & 0.006919 & 0.457500 & 0.003170 & 0.705500 & 0.001123 & 0.950000 & -0.002210 \\
\hline 0.210000 & -0.007036 & 0.468500 & 0.004114 & 0.710500 & -0.001156 & 0.961500 & -0.001612 \\
\hline 0.226000 & -0.007127 & 0.478500 & -0.005147 & 0.725000 & 0.001196 & 0.970500 & -0.001149 \\
\hline 0.232500 & -0.007082 & 0.488500 & 0.005623 & 0.736000 & -0.001251 & 0.982500 & -0.000997 \\
\hline 0.245500 & -0.007120 & 0.491500 & 0.005822 & 0.748500 & -0.001456 & 0.990000 & 0.000902 \\
\hline
\end{tabular}




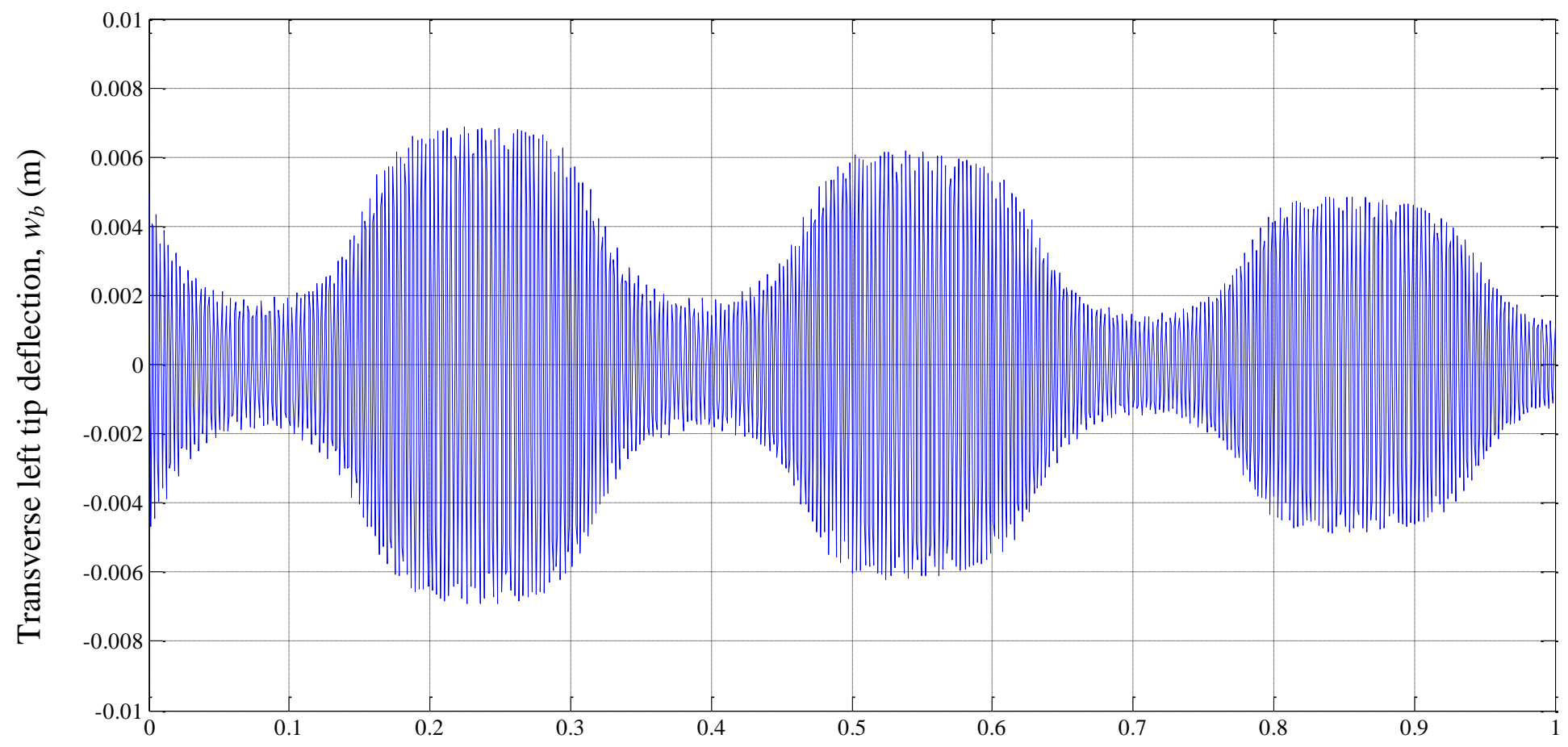

Time (s)

Figure 6.49 Transverse tip deflection at the left end, $w_{b}$, FSDT, $\Omega=20 \mathrm{rad} / \mathrm{s},[0 / \pm 45]_{\mathrm{s}}$ 


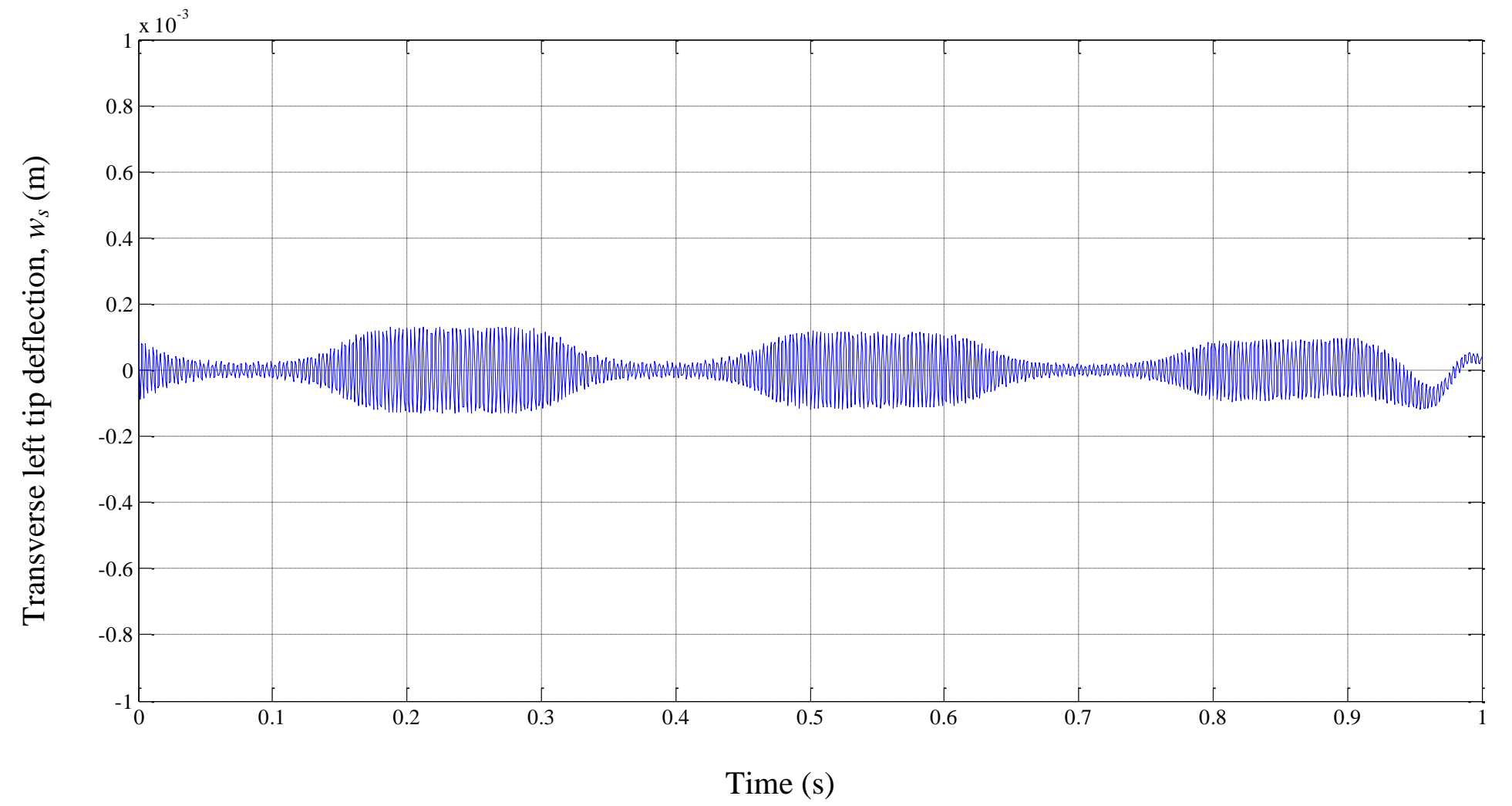

Figure 6.50 Transverse tip deflection at the left end, $w_{s}$, FSDT, $\Omega=20 \mathrm{rad} / \mathrm{s},[0 / \pm 45]_{s}$ 


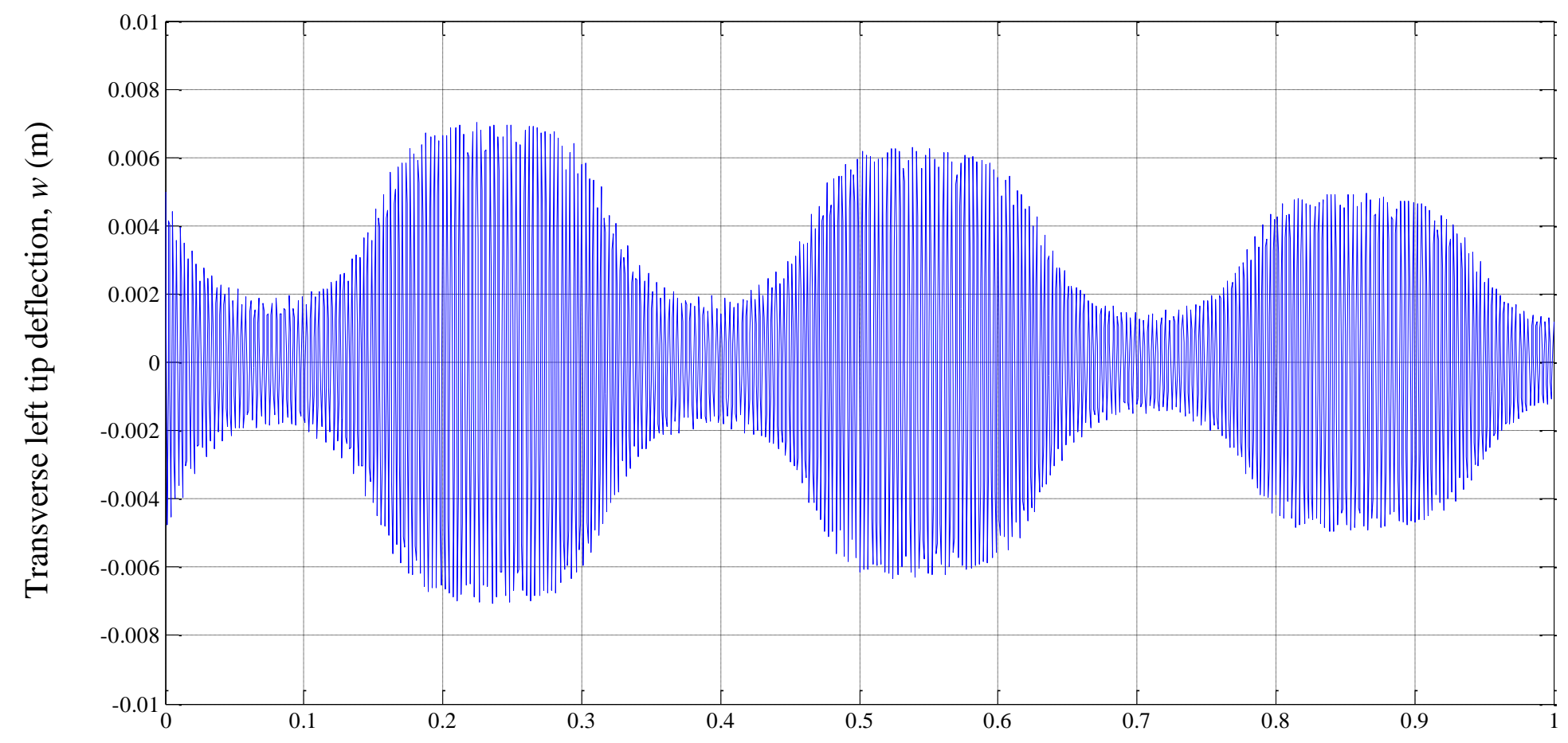

Time (s)

Figure 6.51 Transverse tip deflection at the left end, $w$, FSDT, $\Omega=20 \mathrm{rad} / \mathrm{s},[0 / \pm 45]_{\mathrm{s}}$ 


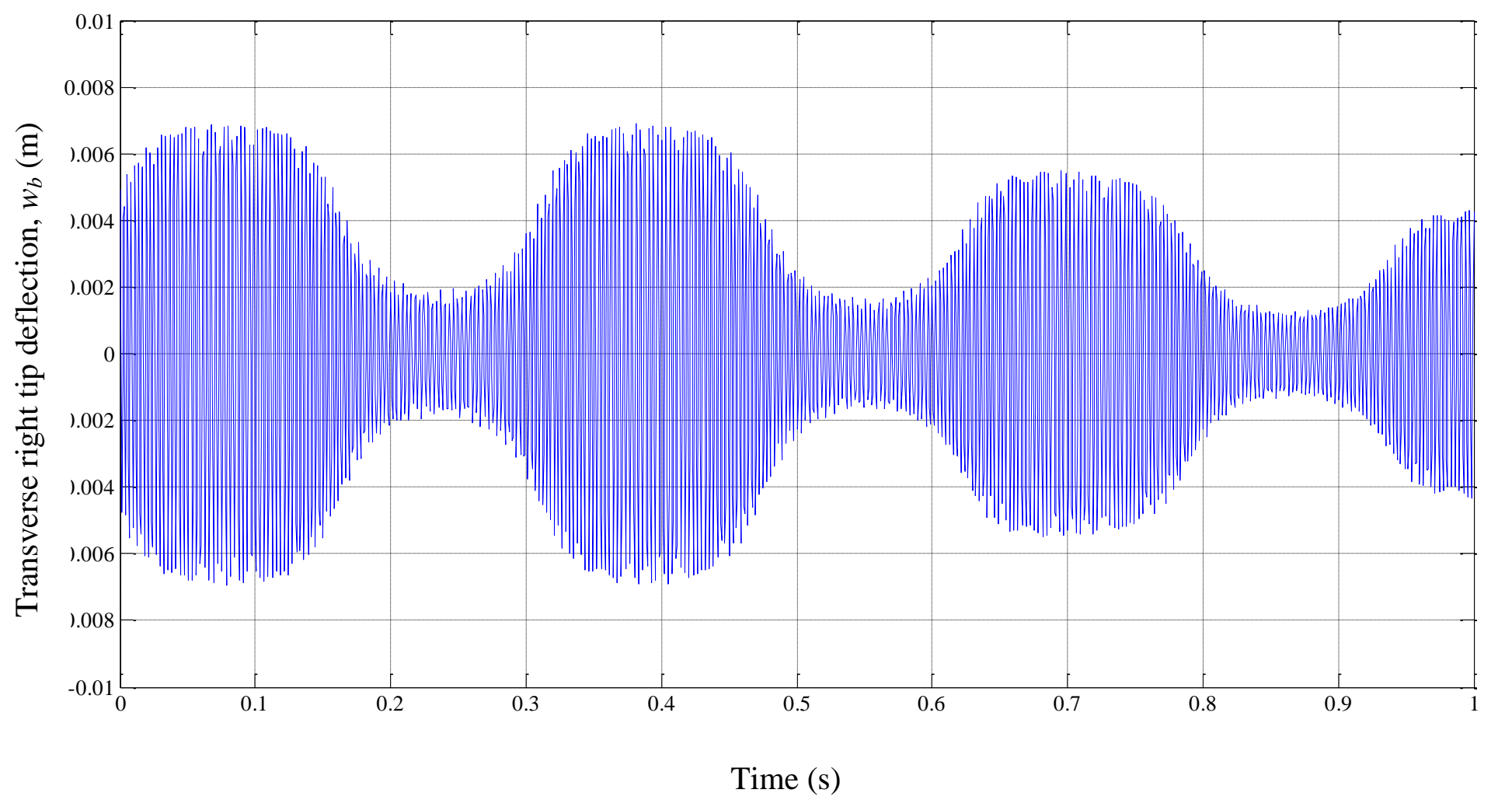

Figure 6.52 Transverse tip deflection at the right end, $w_{b}$, FSDT, $\Omega=20 \mathrm{rad} / \mathrm{s},[0 / \pm 45]_{\mathrm{s}}$ 


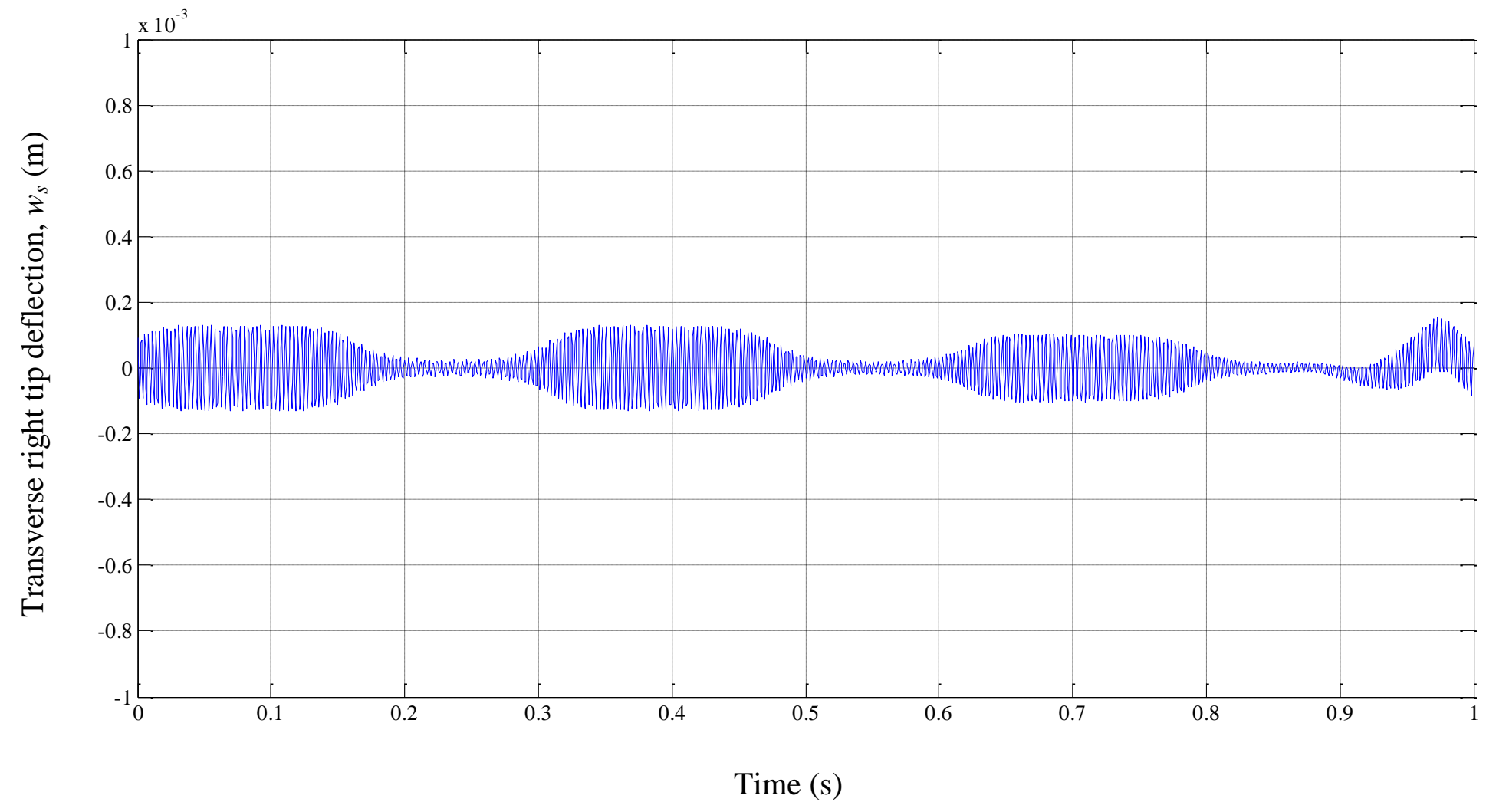

Figure 6.53 Transverse tip deflection at the right end, $w_{s}$, FSDT, $\Omega=20 \mathrm{rad} / \mathrm{s},[0 / \pm 45]_{\mathrm{s}}$ 


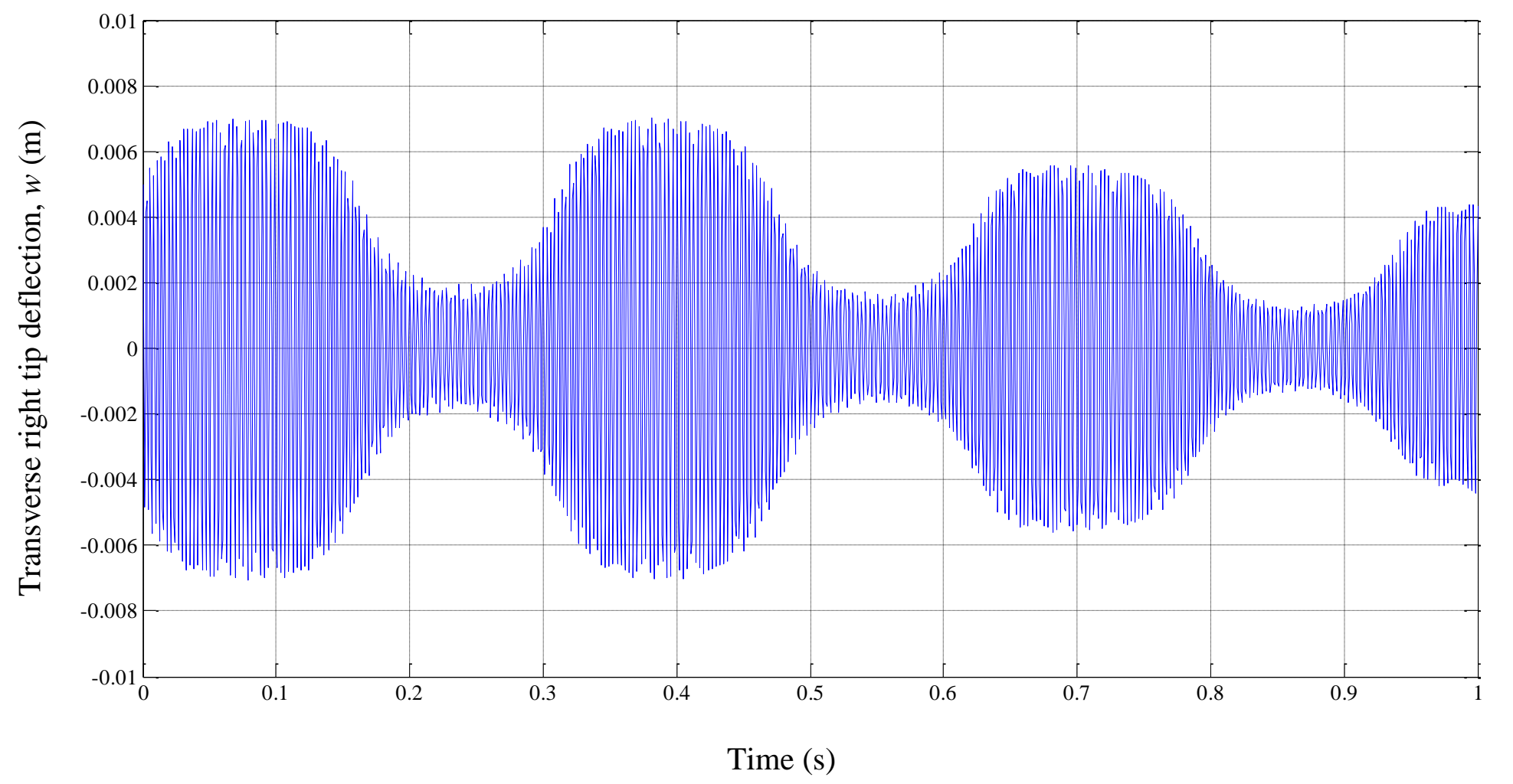

Figure 6.54 Transverse tip deflection at the right end, $w_{s}$, FSDT, $\Omega=20 \mathrm{rad} / \mathrm{s},[0 / \pm 45]_{s}$ 


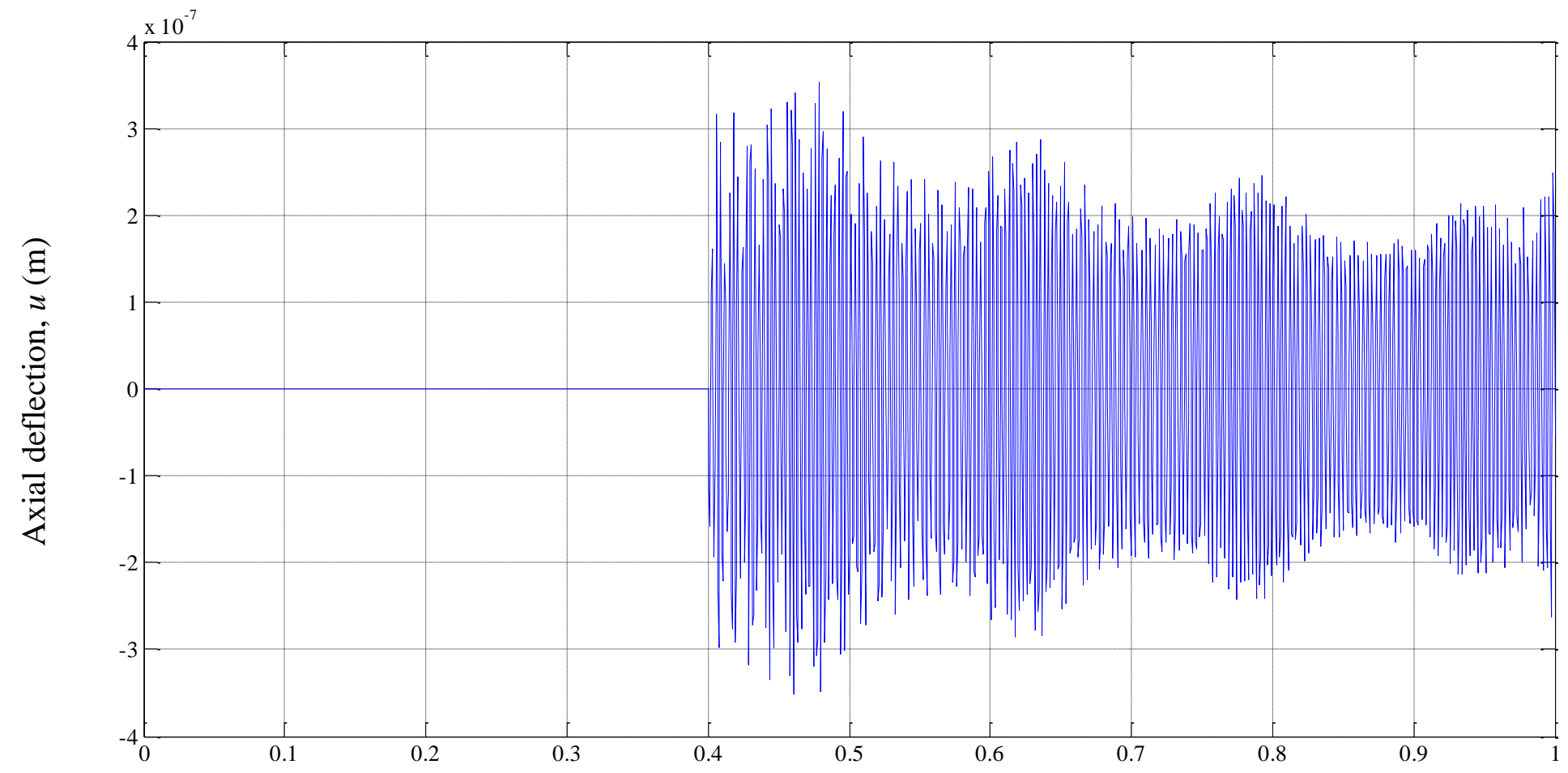

Time (s)

Figure 6.55 Axial deflection at the right end, $u$, FSDT, $\Omega=20 \mathrm{rad} / \mathrm{s},[0 / \pm 45]_{\mathrm{s}}$ 
Table 6.16 Transverse tip deflection at the left end, $w$, FSDT, $\Omega=20 \mathrm{rad} / \mathrm{s},[0 / \pm 45]_{\mathrm{s}}$

\begin{tabular}{|c|c|c|c|c|c|c|c|}
\hline$t(\mathrm{~s})$ & $w(\mathrm{~m})$ & $t(\mathrm{~s})$ & $w(\mathrm{~m})$ & $t(\mathrm{~s})$ & $w(\mathrm{~m})$ & $t(\mathrm{~s})$ & $w(\mathrm{~m})$ \\
\hline 0.000000 & 0.005000 & 0.259000 & 0.006805 & 0.502000 & 0.006175 & 0.752500 & -0.001994 \\
\hline 0.011000 & 0.003952 & 0.263500 & -0.006980 & 0.518500 & -0.006124 & 0.769500 & -0.002483 \\
\hline 0.021000 & -0.003266 & 0.274000 & 0.006765 & 0.524500 & -0.006340 & 0.779500 & 0.003284 \\
\hline 0.035500 & -0.002542 & 0.280000 & 0.006770 & 0.538500 & 0.006311 & 0.789000 & -0.003873 \\
\hline 0.041500 & -0.002306 & 0.294500 & 0.006396 & 0.540000 & -0.006295 & 0.797500 & -0.004418 \\
\hline 0.052000 & 0.002160 & 0.301500 & -0.005959 & 0.551000 & 0.006240 & 0.804500 & 0.004631 \\
\hline 0.066000 & -0.001904 & 0.314000 & 0.005156 & 0.562000 & -0.006233 & 0.814500 & -0.004836 \\
\hline 0.075500 & -0.001855 & 0.321000 & -0.004098 & 0.576000 & 0.006059 & 0.820500 & -0.004731 \\
\hline 0.089500 & 0.001973 & 0.333500 & 0.003423 & 0.582000 & 0.006027 & 0.838500 & -0.004939 \\
\hline 0.099000 & 0.001936 & 0.342000 & 0.002836 & 0.592500 & -0.005858 & 0.841500 & -0.004953 \\
\hline 0.109500 & -0.002232 & 0.354000 & 0.002351 & 0.607000 & -0.005506 & 0.854000 & -0.004929 \\
\hline 0.115500 & -0.002352 & 0.360000 & 0.002201 & 0.615500 & -0.005163 & 0.865000 & 0.004936 \\
\hline 0.127500 & -0.002788 & 0.370500 & -0.002068 & 0.621000 & -0.004632 & 0.876000 & -0.004854 \\
\hline 0.136000 & -0.003253 & 0.380000 & -0.001959 & 0.633500 & 0.003705 & 0.880500 & 0.004692 \\
\hline 0.144500 & -0.003926 & 0.394000 & 0.001942 & 0.640500 & -0.003020 & 0.894500 & -0.004752 \\
\hline 0.157000 & 0.004895 & 0.408000 & -0.001948 & 0.654500 & -0.002335 & 0.900500 & -0.004675 \\
\hline 0.169500 & -0.005877 & 0.417500 & -0.002065 & 0.660500 & -0.002173 & 0.911000 & 0.004449 \\
\hline 0.176500 & 0.006243 & 0.428000 & 0.002275 & 0.672500 & -0.001758 & 0.921000 & -0.004338 \\
\hline 0.188000 & 0.006727 & 0.434000 & 0.002450 & 0.683000 & 0.001656 & 0.931000 & 0.003767 \\
\hline 0.194000 & 0.006654 & 0.448500 & 0.002968 & 0.697000 & -0.001485 & 0.940500 & -0.003437 \\
\hline 0.206000 & 0.006881 & 0.458500 & -0.003547 & 0.706500 & -0.001481 & 0.951500 & -0.002615 \\
\hline 0.210500 & -0.006976 & 0.465500 & 0.004342 & 0.716000 & -0.001439 & 0.960000 & -0.002273 \\
\hline 0.226000 & -0.007032 & 0.476500 & 0.005261 & 0.720500 & 0.001520 & 0.971500 & -0.001748 \\
\hline 0.235500 & -0.007046 & 0.489000 & -0.005688 & 0.736000 & 0.001654 & 0.980500 & -0.001418 \\
\hline 0.248000 & -0.007037 & 0.499000 & 0.005966 & 0.746500 & -0.001847 & 0.991000 & 0.001348 \\
\hline
\end{tabular}




\subsubsection{Moving beam simulation using HSDT}

The response of the beam formulated using HSDT is presented in this section. All the five cases presented for CLPT and FSDT are considered here. The gains that are supplied in CLPT formulation are used for HSDT. It is observed that damping effect is less compared to the CLPT and the amplitudes are higher than the initial deflection by the end of 1 second.

The response of the $[0 / \pm 45]$ beam with axial oscillation frequencies of $10 \mathrm{rad} / \mathrm{s}$ and 20 $\mathrm{rad} / \mathrm{s}$ are shown in Figures 6.56 - 6.69. Tables 6.18 and 6.19 present the tip deflections at the left end of the beam. The responses of the $[0 / \pm 20]$ beam with axial frequencies of $10 \mathrm{rad} / \mathrm{s}$ and 20 $\mathrm{rad} / \mathrm{s}$ are shown from Figures $6.70-6.83$. Tables 6.20 and 6.21 present the tip deflections at the left end of the beam. The response of $[0 / \pm 45]_{\mathrm{s}}$ beam with an axial oscillation frequency of 20 $\mathrm{rad} / \mathrm{s}$ is shown in Figures $6.84-6.90$. Table 6.22 presents the tip deflection at the left end of the beam. In HSDT the transverse displacements are composed of bending and shear components. The bending and shear components of the transverse tip displacements at the right and the left end are shown separately for all the cases.

The magnification factors for the tip deflection due to the axial oscillation of the beam are presented in Table 6.17. It is observed that the magnification factors range between 40 and 43\% and are higher than the corresponding CLPT and FSDT cases.

Table 6.17 Magnification factors for the moving beams with HSDT formulation

\begin{tabular}{|c|c|c|c|c|}
\hline Lay up & frequency & $w_{\max }^{\text {static }}$ & $w_{\max }^{\text {moving }}$ & $\begin{array}{c}\text { Mag. factor } \\
\text { moving } \\
=\frac{w_{\max }^{\text {sax }}}{w_{\max }^{\text {static }}}\end{array}$ \\
\hline$[0 / \pm 20]$ & 10 & 0.005 & 0.007017 & 1.4034 \\
\hline$[0 / \pm 20]$ & 20 & 0.005 & 0.007139 & 1.4278 \\
\hline$[0 / \pm 45]$ & 10 & 0.005 & 0.007002 & 1.4004 \\
\hline$[0 / \pm 45]$ & 20 & 0.005 & 0.007069 & 1.4138 \\
\hline$[0 / \pm 45]_{\mathrm{s}}$ & 20 & 0.005 & 0.007048 & 1.4066 \\
\hline
\end{tabular}




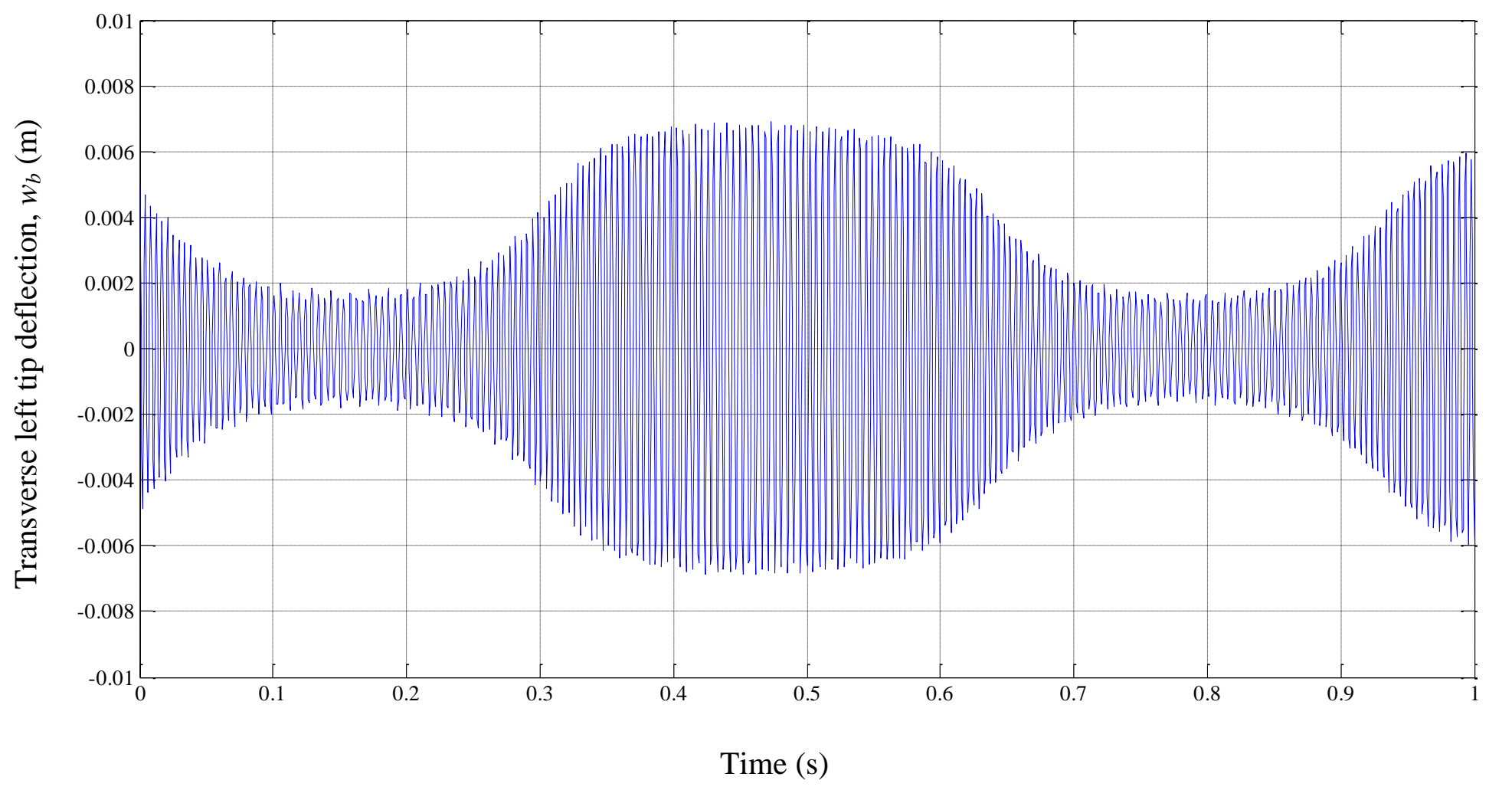

Figure 6.56 Transverse tip deflection at the left end, $w_{b}, \mathrm{HSDT}, \Omega=10 \mathrm{rad} / \mathrm{s},[0 / \pm 45]$ 


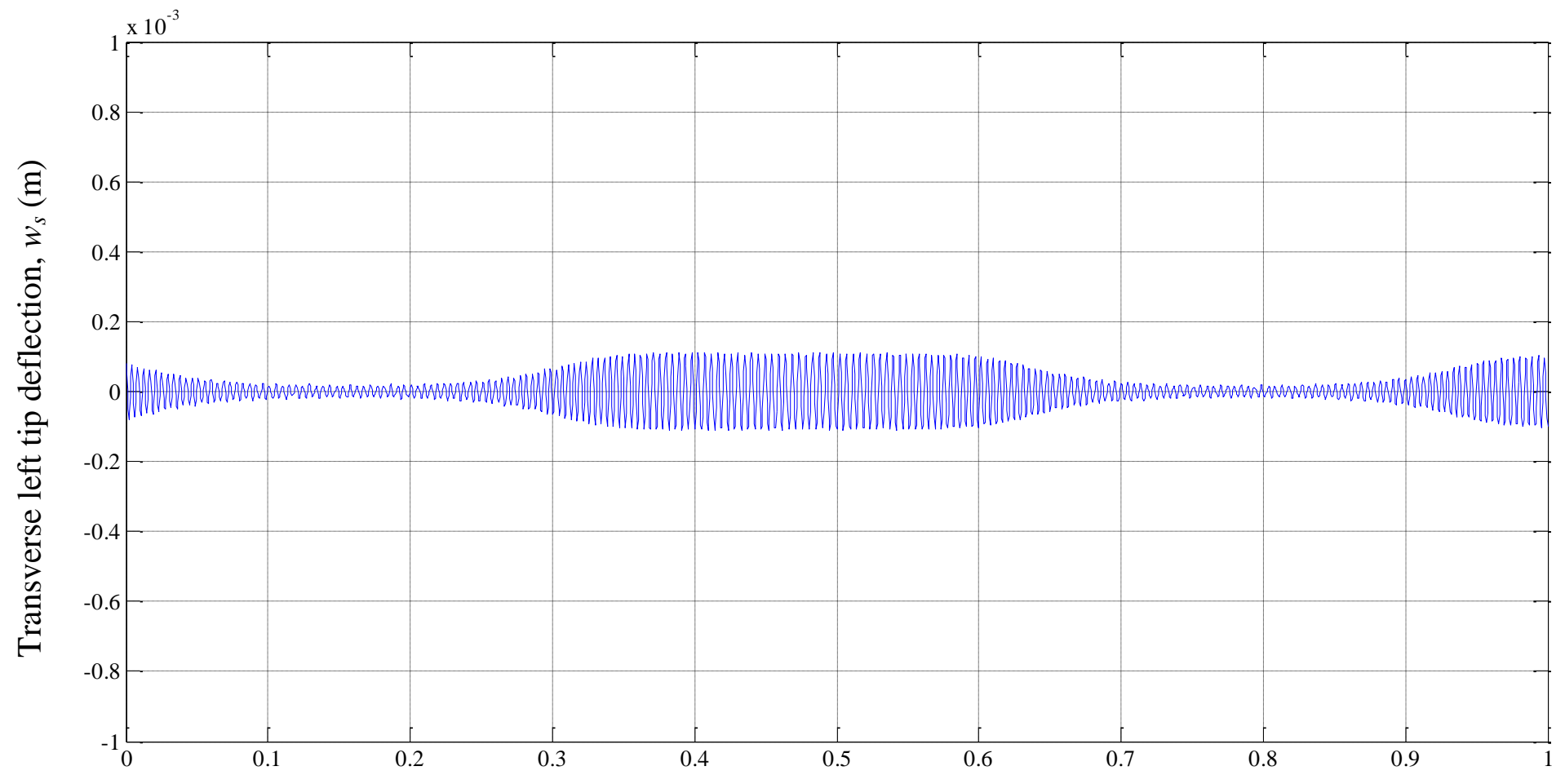

Time (s)

Figure 6.57 Transverse tip deflection at the left end, $w_{s}, \mathrm{HSDT}, \Omega=10 \mathrm{rad} / \mathrm{s},[0 / \pm 45]$ 


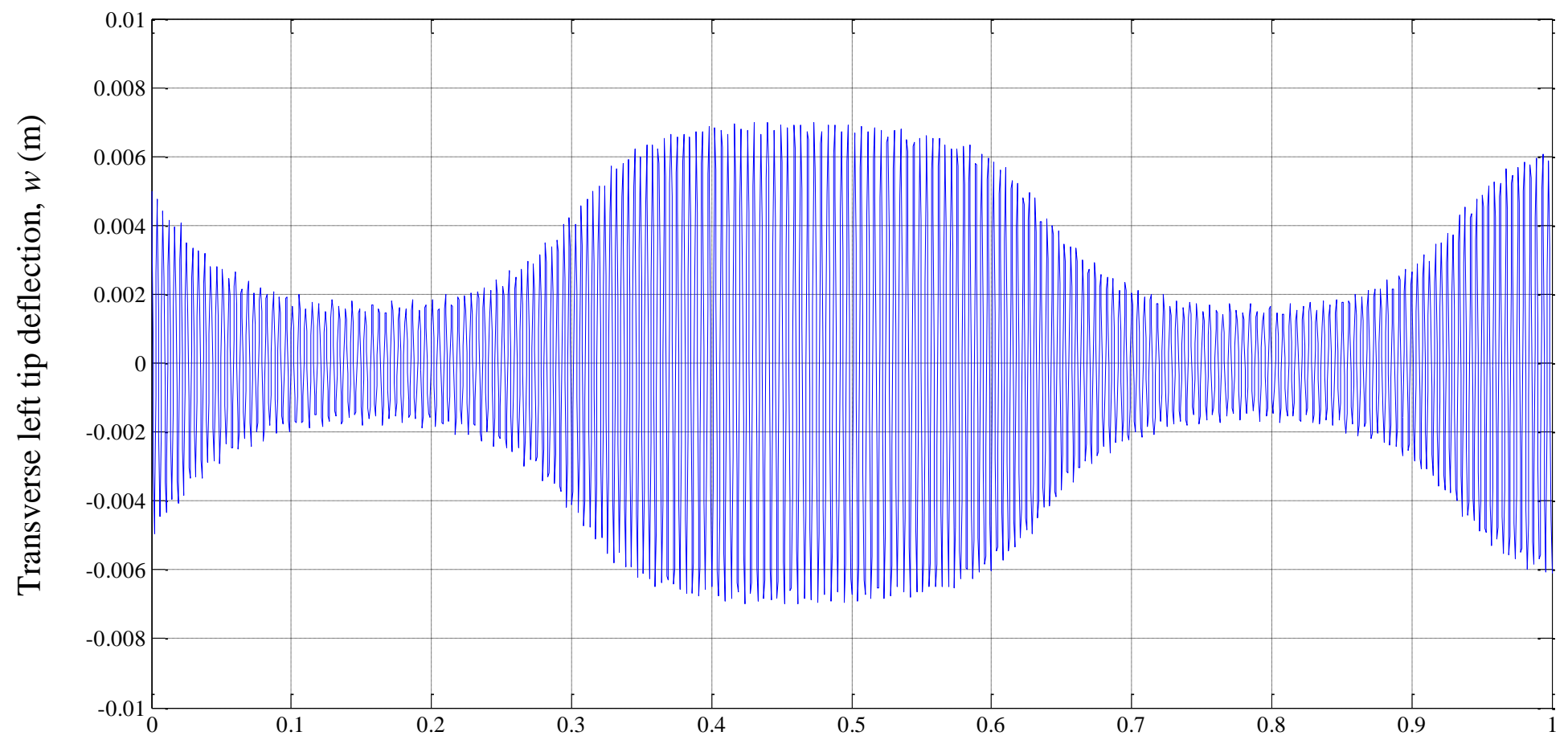

Time (s)

Figure 6.58 Transverse tip deflection at the left end, $w$, HSDT, $\Omega=10 \mathrm{rad} / \mathrm{s},[0 / \pm 45]$ 


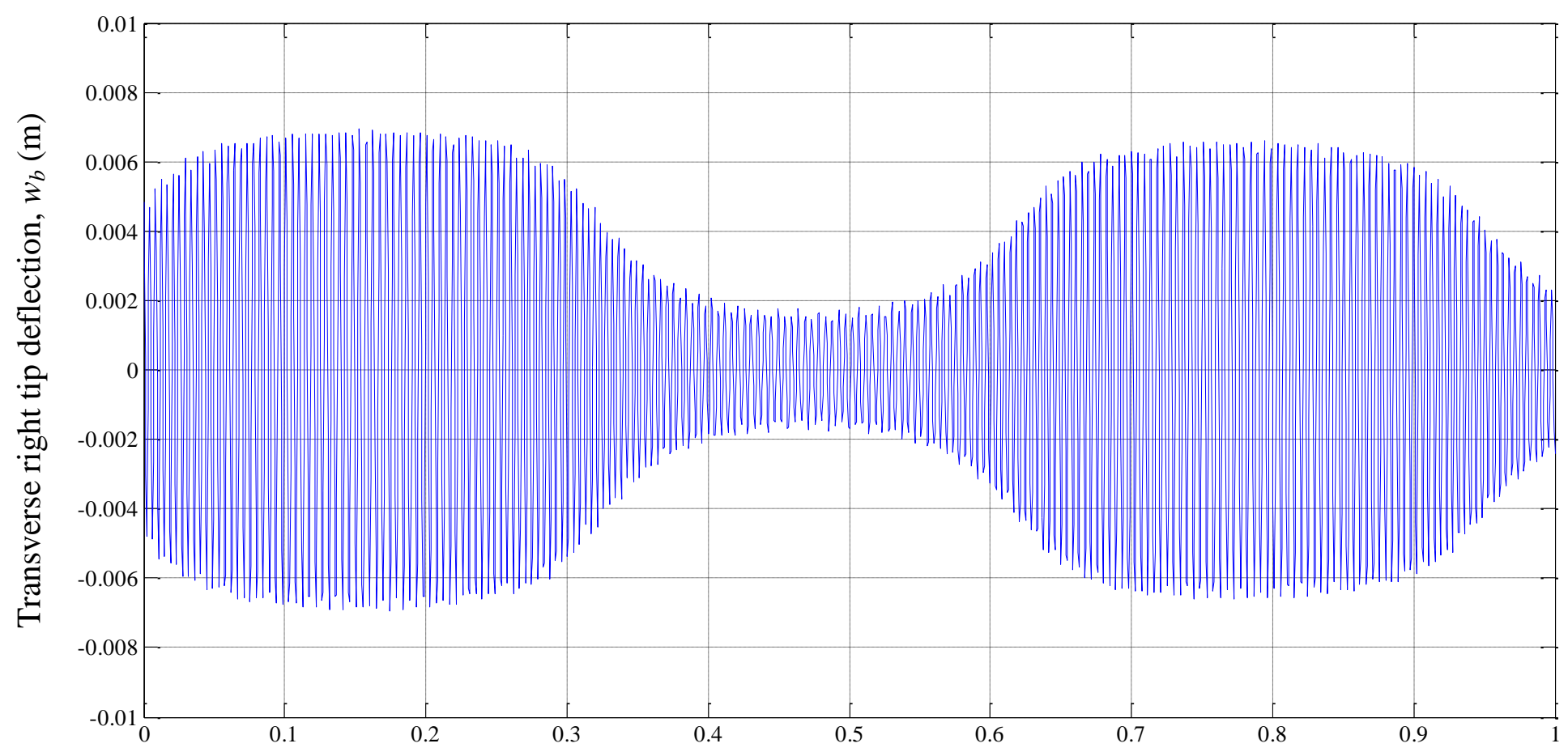

Time (s)

Figure 6.59 Transverse tip deflection at the right end, $w_{b}$, HSDT, $\Omega=10 \mathrm{rad} / \mathrm{s},[0 / \pm 45]$ 


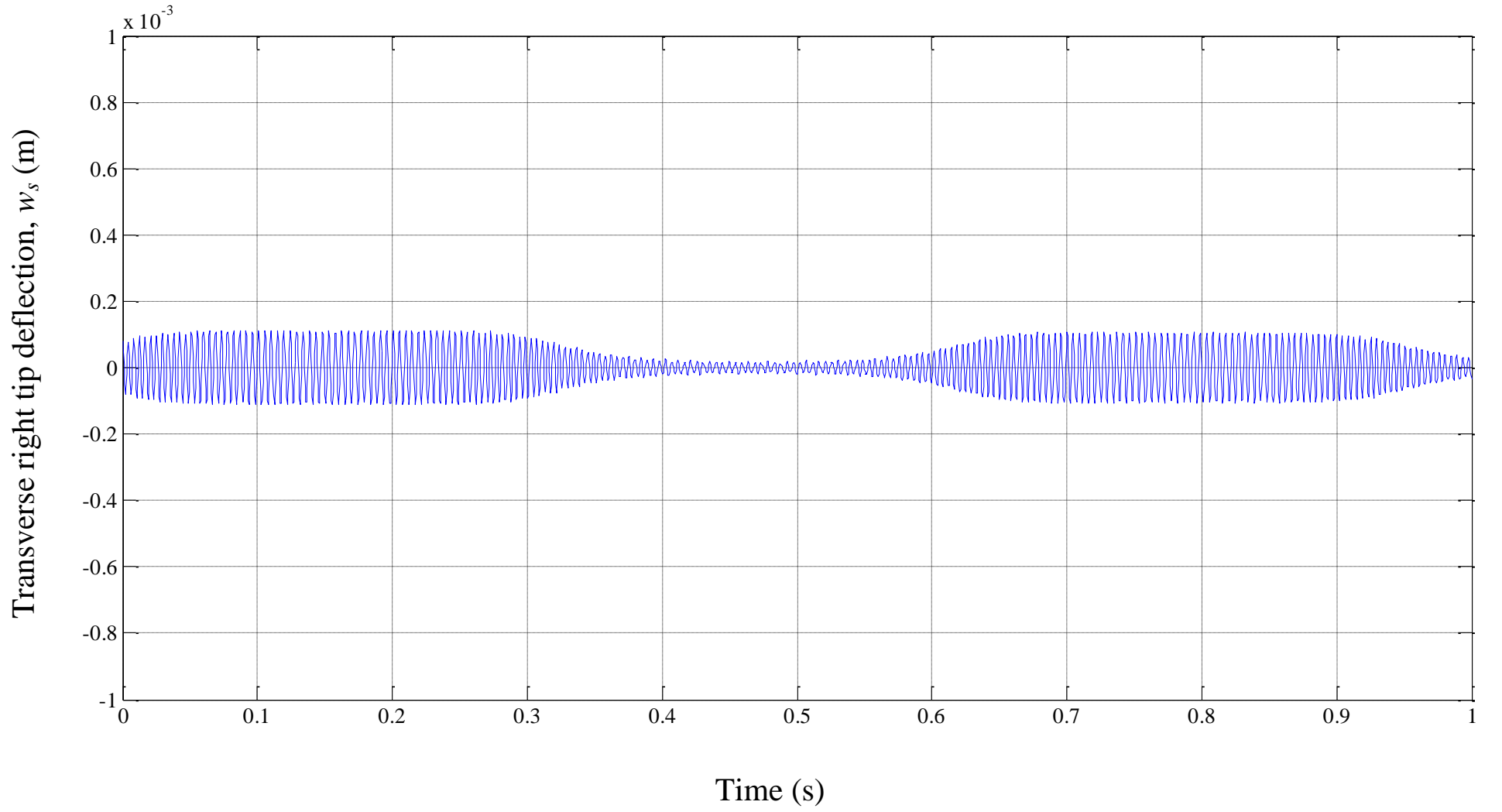

Figure 6.60 Transverse tip deflection at the right end, $w_{s}, \mathrm{HSDT}, \Omega=10 \mathrm{rad} / \mathrm{s},[0 / \pm 45]$ 


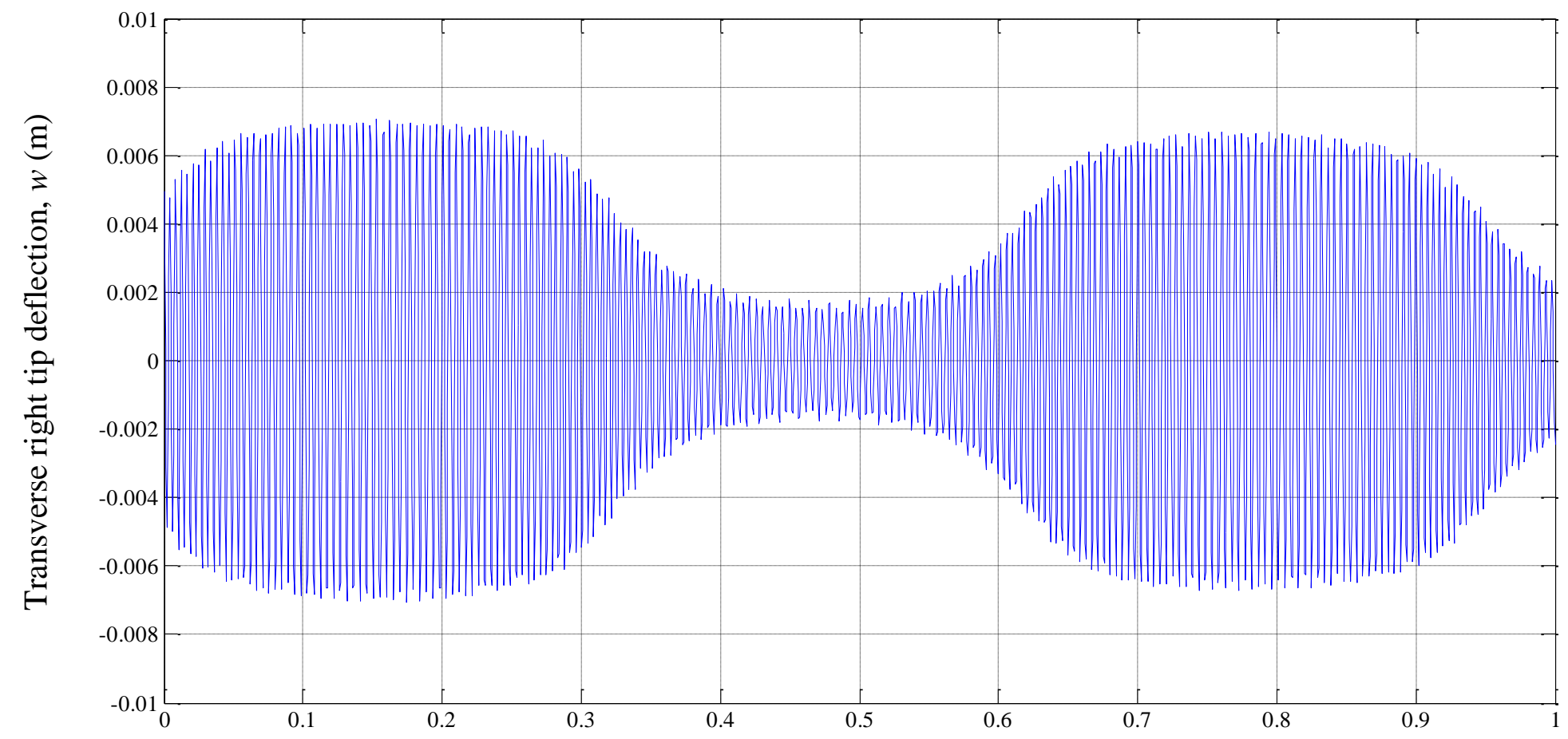

Time (s)

Figure 6.61 Transverse tip deflection at the right end, $w$, HSDT, $\Omega=10 \mathrm{rad} / \mathrm{s},[0 / \pm 45]$ 


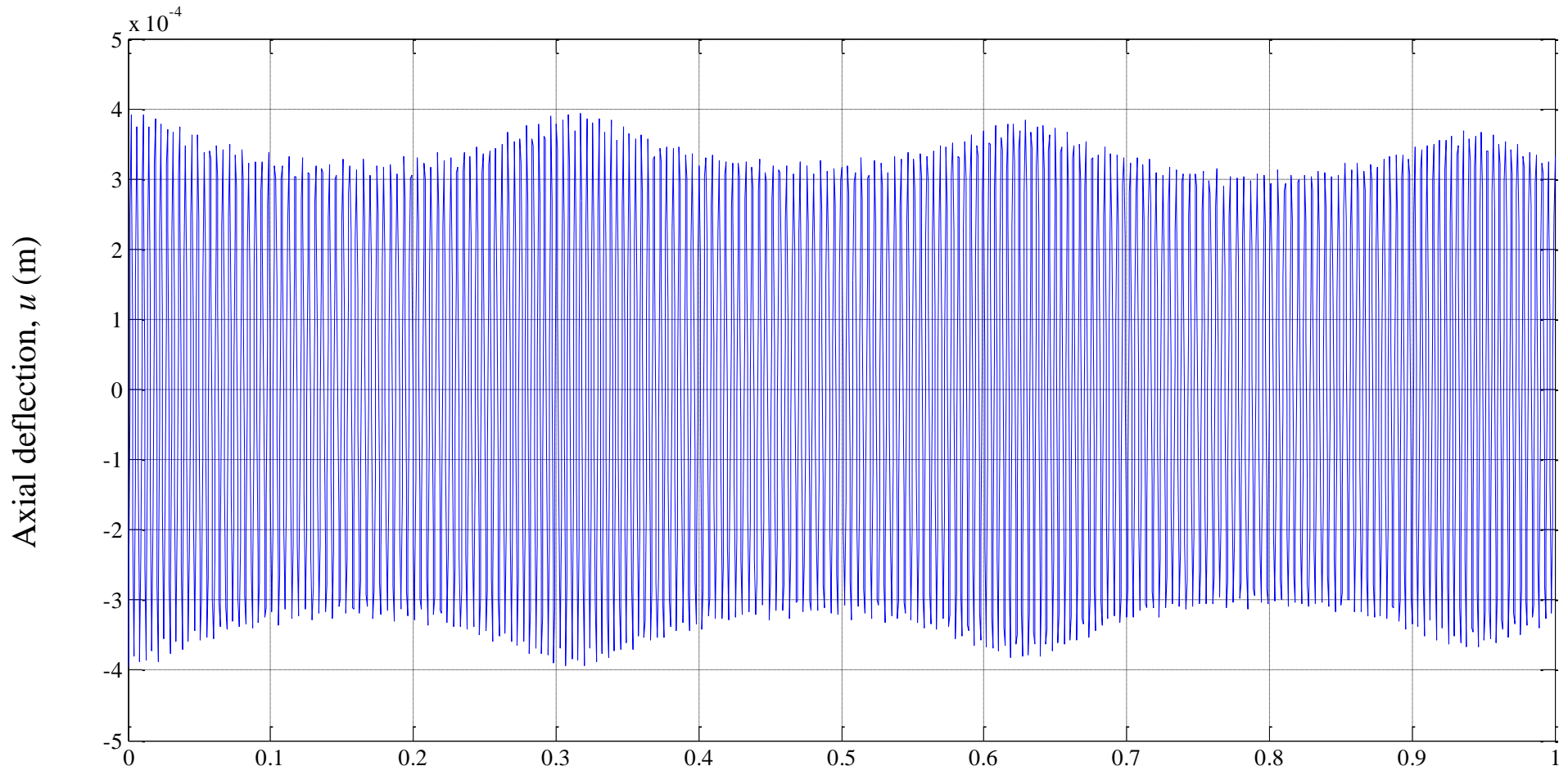

Time (s)

Figure 6.62 Axial deflection at the right end, $u$, HSDT, $\Omega=10 \mathrm{rad} / \mathrm{s},[0 / \pm 45]$ 
Table 6.18 Transverse tip deflection at the left end, $w$, HSDT, $\Omega=10 \mathrm{rad} / \mathrm{s},[0 / \pm 45]$

\begin{tabular}{|c|c|c|c|c|c|c|c|}
\hline$t(\mathrm{~s})$ & $w(\mathrm{~m})$ & $t(\mathrm{~s})$ & $w(\mathrm{~m})$ & $t(\mathrm{~s})$ & $w(\mathrm{~m})$ & $t(\mathrm{~s})$ & $w(\mathrm{~m})$ \\
\hline 0.000000 & 0.005000 & 0.255500 & 0.002694 & 0.504500 & -0.006897 & 0.756000 & 0.001677 \\
\hline 0.010500 & -0.004326 & 0.266500 & -0.002977 & 0.514000 & -0.006835 & 0.763000 & -0.001741 \\
\hline 0.021000 & 0.004052 & 0.279500 & -0.003430 & 0.523500 & -0.006814 & 0.770000 & 0.001717 \\
\hline 0.036000 & -0.003354 & 0.288000 & -0.003485 & 0.535000 & 0.006796 & 0.784500 & 0.001710 \\
\hline 0.049000 & -0.002905 & 0.298500 & 0.004210 & 0.542000 & -0.006791 & 0.791500 & -0.001685 \\
\hline 0.051000 & 0.002741 & 0.309000 & -0.004736 & 0.551000 & -0.006640 & 0.806000 & -0.001736 \\
\hline 0.060000 & 0.002636 & 0.319500 & 0.005127 & 0.562500 & 0.006541 & 0.813000 & 0.001725 \\
\hline 0.071000 & -0.002413 & 0.328000 & 0.005738 & 0.573500 & -0.006509 & 0.827500 & 0.001780 \\
\hline 0.080000 & -0.002264 & 0.338500 & -0.005931 & 0.584500 & 0.006327 & 0.836500 & 0.001795 \\
\hline 0.098500 & -0.002013 & 0.347000 & -0.006233 & 0.593000 & 0.006082 & 0.846000 & 0.001845 \\
\hline 0.105500 & 0.002005 & 0.353500 & 0.006343 & 0.601500 & 0.005833 & 0.853000 & -0.002027 \\
\hline 0.112500 & -0.001891 & 0.366500 & 0.006541 & 0.610000 & 0.005665 & 0.862000 & -0.002111 \\
\hline 0.129000 & 0.001852 & 0.371000 & 0.006653 & 0.620500 & -0.005055 & 0.871000 & -0.002197 \\
\hline 0.136000 & -0.001757 & 0.389000 & 0.006722 & 0.631000 & 0.004781 & 0.889000 & -0.002413 \\
\hline 0.143500 & 0.001798 & 0.398000 & 0.006886 & 0.641500 & -0.004133 & 0.895500 & 0.002719 \\
\hline 0.150500 & -0.001808 & 0.409500 & -0.006903 & 0.650000 & -0.003704 & 0.908500 & 0.003147 \\
\hline 0.165000 & -0.001801 & 0.416500 & 0.006952 & 0.660500 & 0.003345 & 0.919000 & -0.003555 \\
\hline 0.172000 & 0.001809 & 0.423500 & -0.006987 & 0.673500 & 0.002934 & 0.927500 & -0.003773 \\
\hline 0.186500 & 0.001861 & 0.430500 & 0.006974 & 0.684500 & -0.002617 & 0.938000 & 0.004516 \\
\hline 0.193500 & -0.001890 & 0.440000 & 0.006988 & 0.695500 & 0.002335 & 0.948500 & -0.004870 \\
\hline 0.203000 & -0.001851 & 0.452000 & -0.006990 & 0.707000 & -0.002152 & 0.957000 & -0.005279 \\
\hline 0.217000 & -0.002056 & 0.461500 & -0.007000 & 0.716000 & -0.002068 & 0.967500 & 0.005652 \\
\hline 0.226000 & -0.002080 & 0.473500 & 0.007002 & 0.723000 & 0.001980 & 0.976000 & 0.005690 \\
\hline 0.235500 & -0.002252 & 0.485500 & -0.006926 & 0.732000 & 0.001814 & 0.982500 & -0.005976 \\
\hline 0.246500 & 0.002438 & 0.495000 & -0.006953 & 0.741500 & 0.001763 & 0.995500 & -0.006077 \\
\hline
\end{tabular}




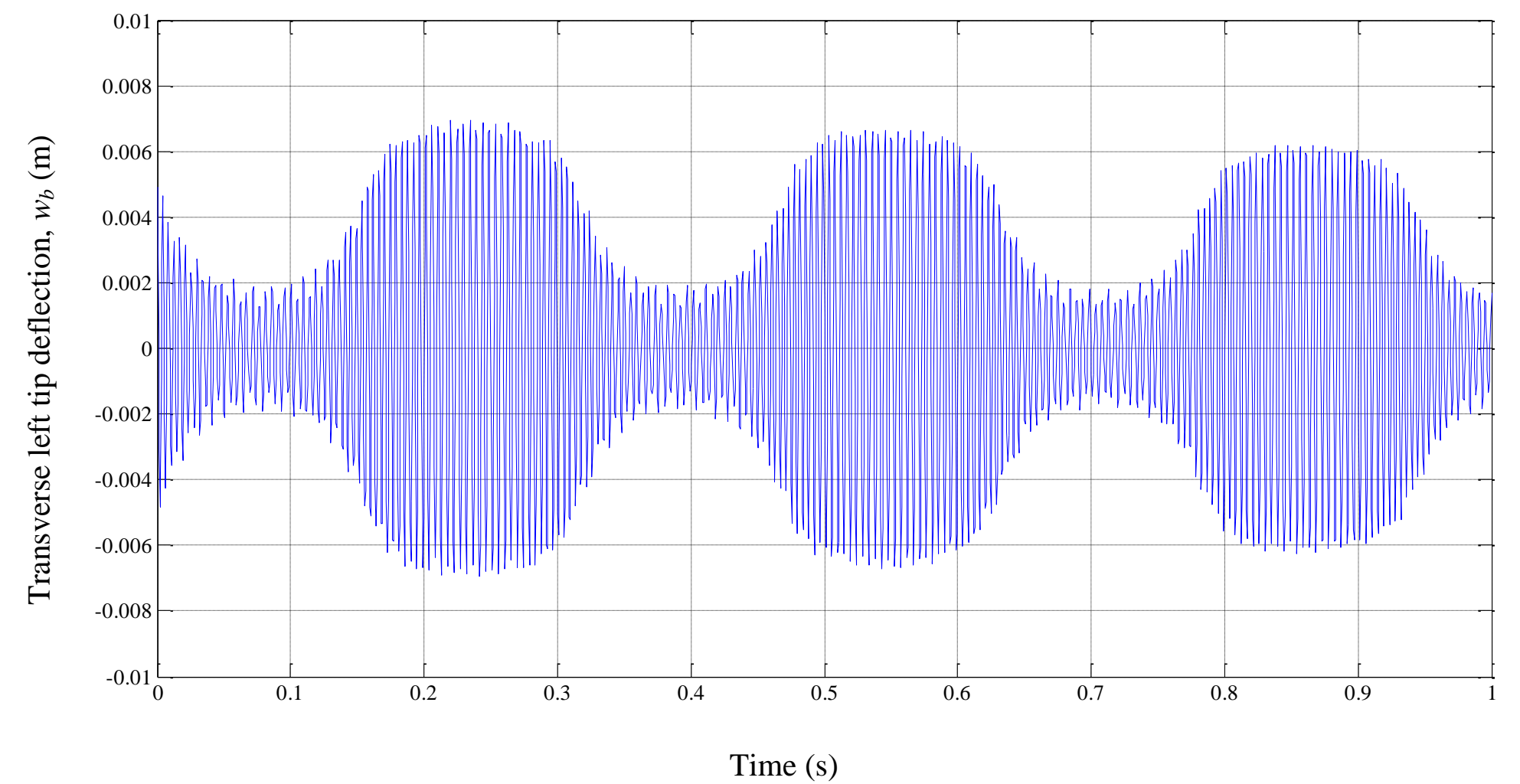

Figure 6.63 Transverse tip deflection at the left end, $w_{b}$, HSDT, $\Omega=20 \mathrm{rad} / \mathrm{s},[0 / \pm 45]$ 


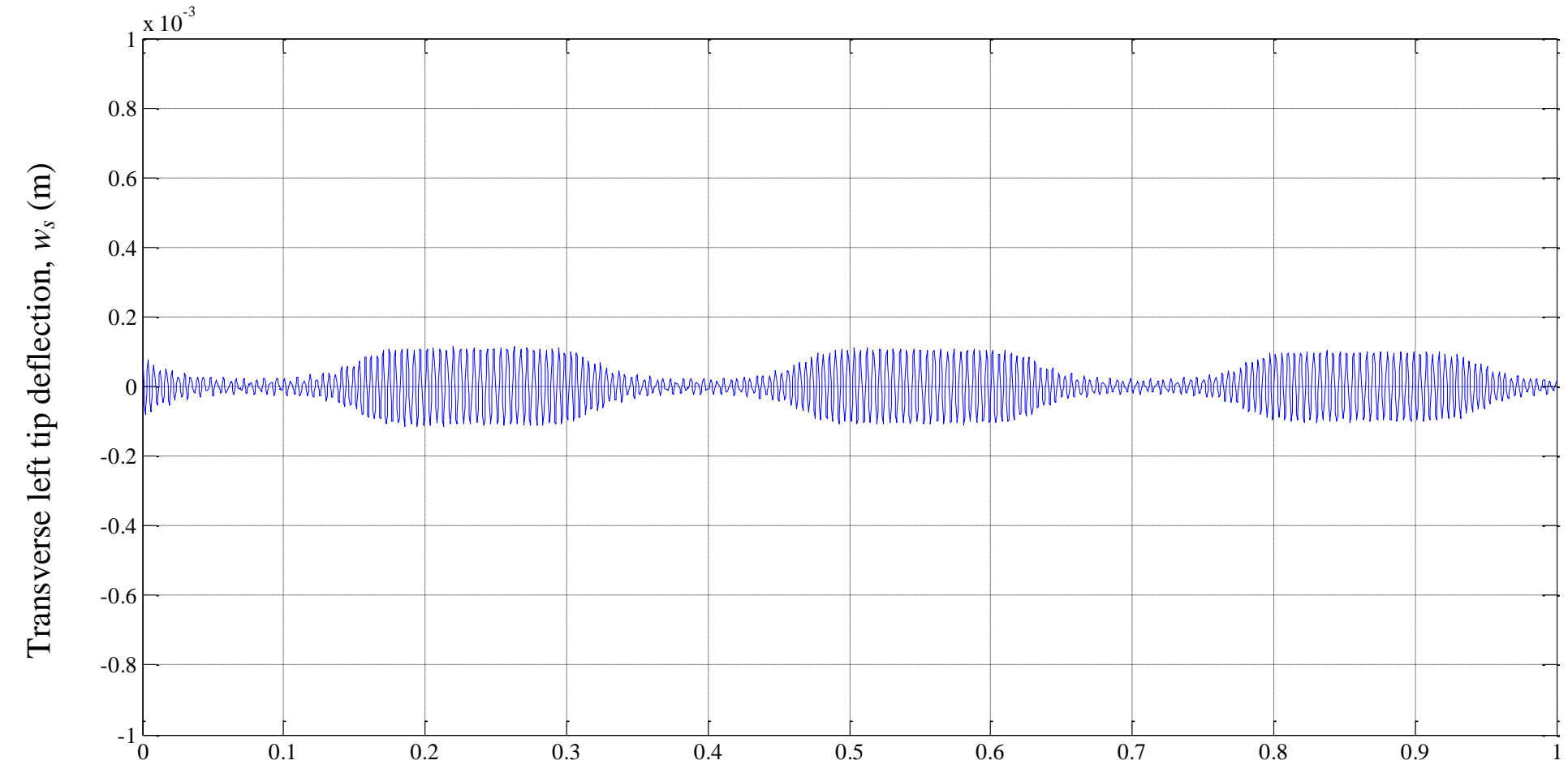

Time (s)

Figure 6.64 Transverse tip deflection at the left end, $w_{s}$, HSDT, $\Omega=20 \mathrm{rad} / \mathrm{s},[0 / \pm 45]$ 


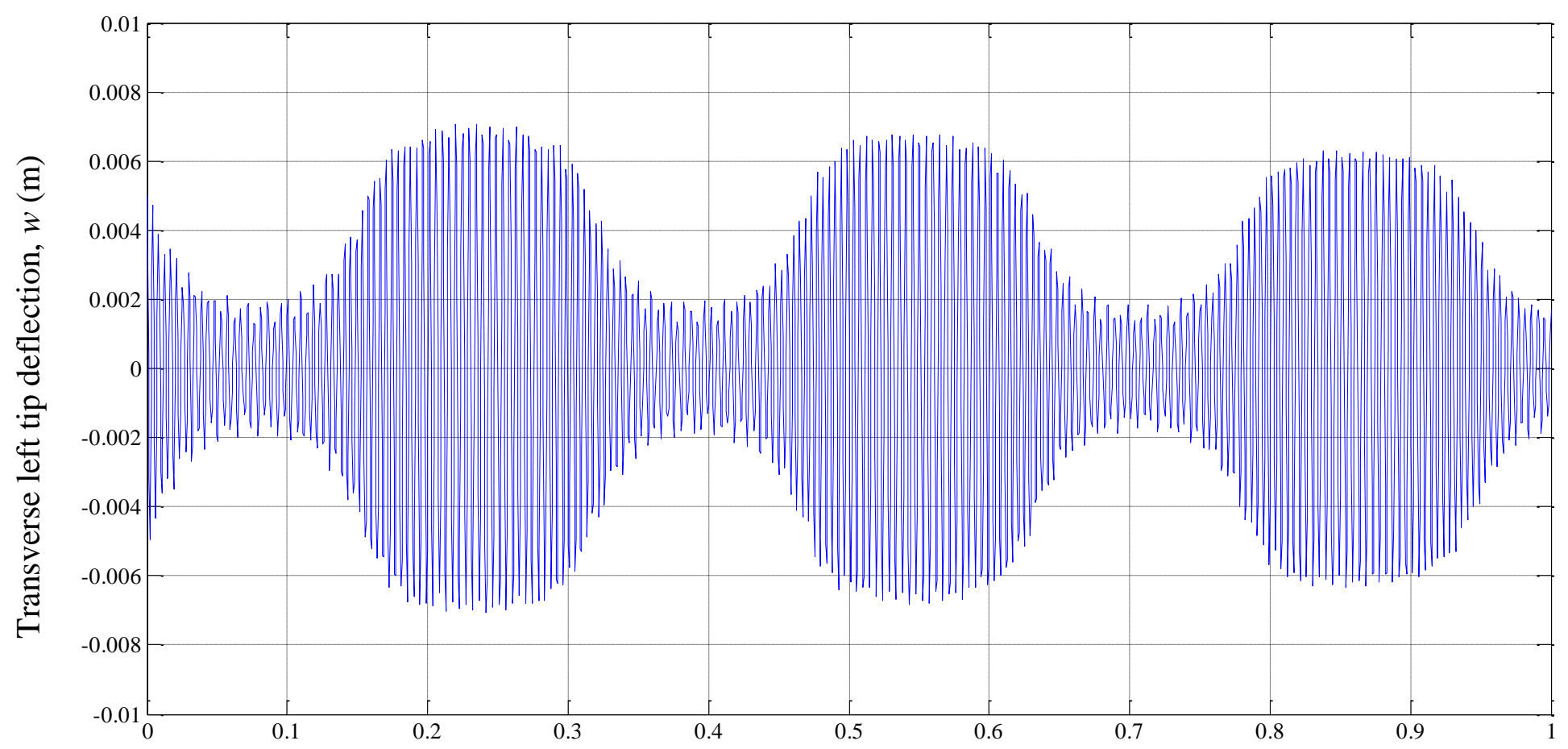

Time (s)

Figure 6.65 Transverse tip deflection at the left end, $w$, HSDT, $\Omega=20 \mathrm{rad} / \mathrm{s},[0 / \pm 45]$ 


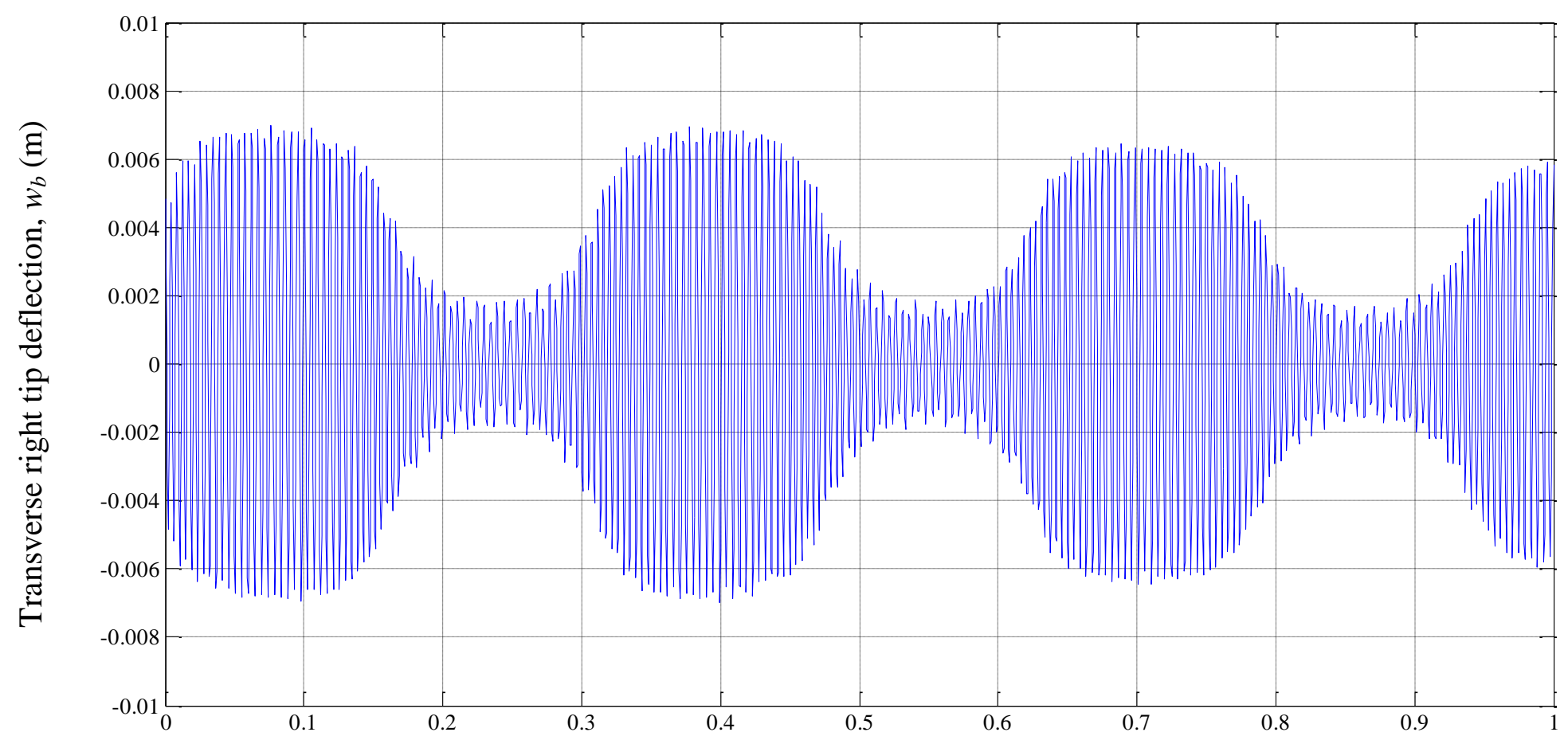

Time (s)

Figure 6.66 Transverse tip deflection at the right end, $w_{b}$, HSDT, $\Omega=20 \mathrm{rad} / \mathrm{s},[0 / \pm 45]$ 


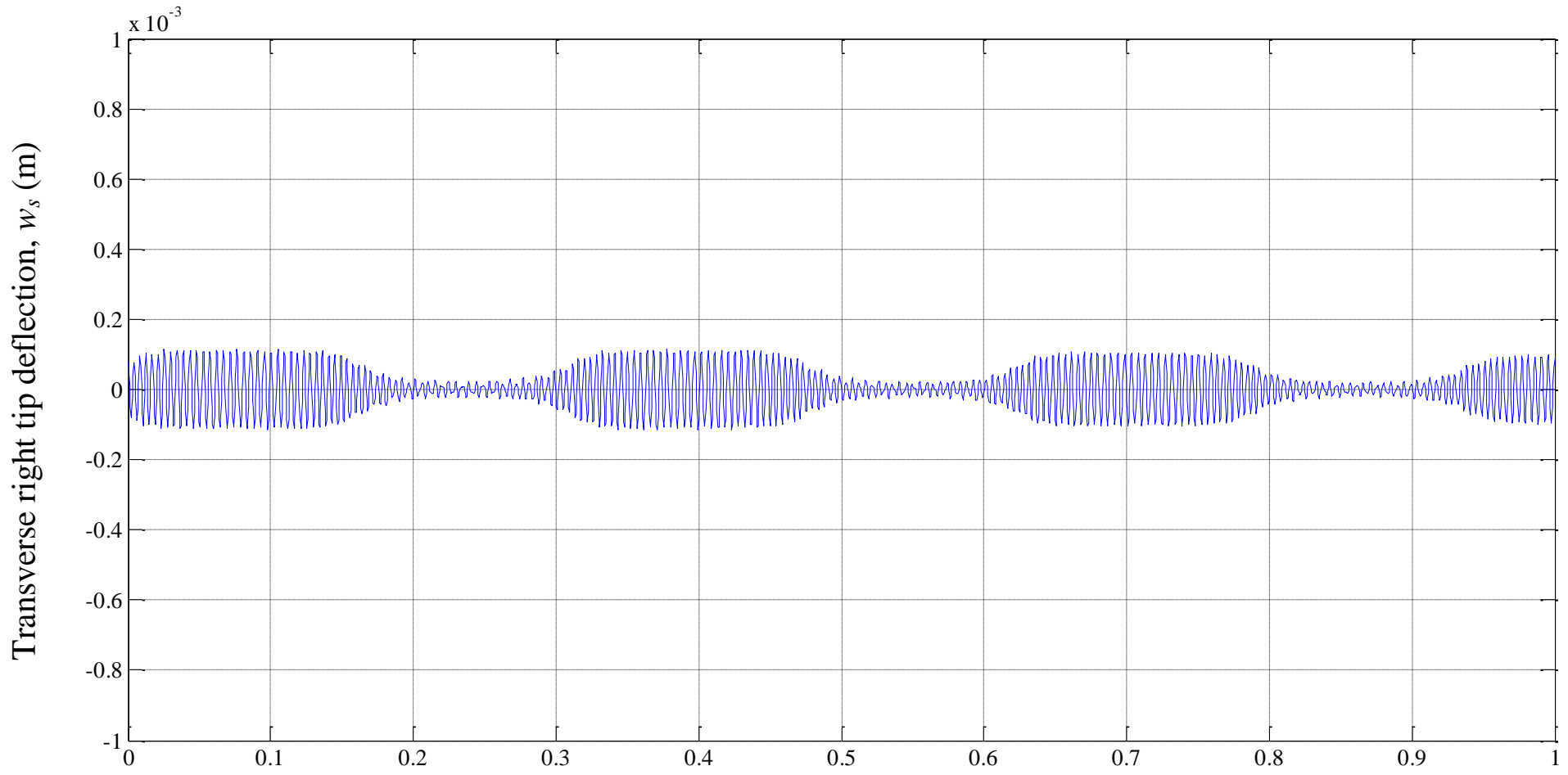

Time (s)

Figure 6.67 Transverse tip deflection at the right end, $w_{s}$, HSDT, $\Omega=20 \mathrm{rad} / \mathrm{s},[0 / \pm 45]$ 


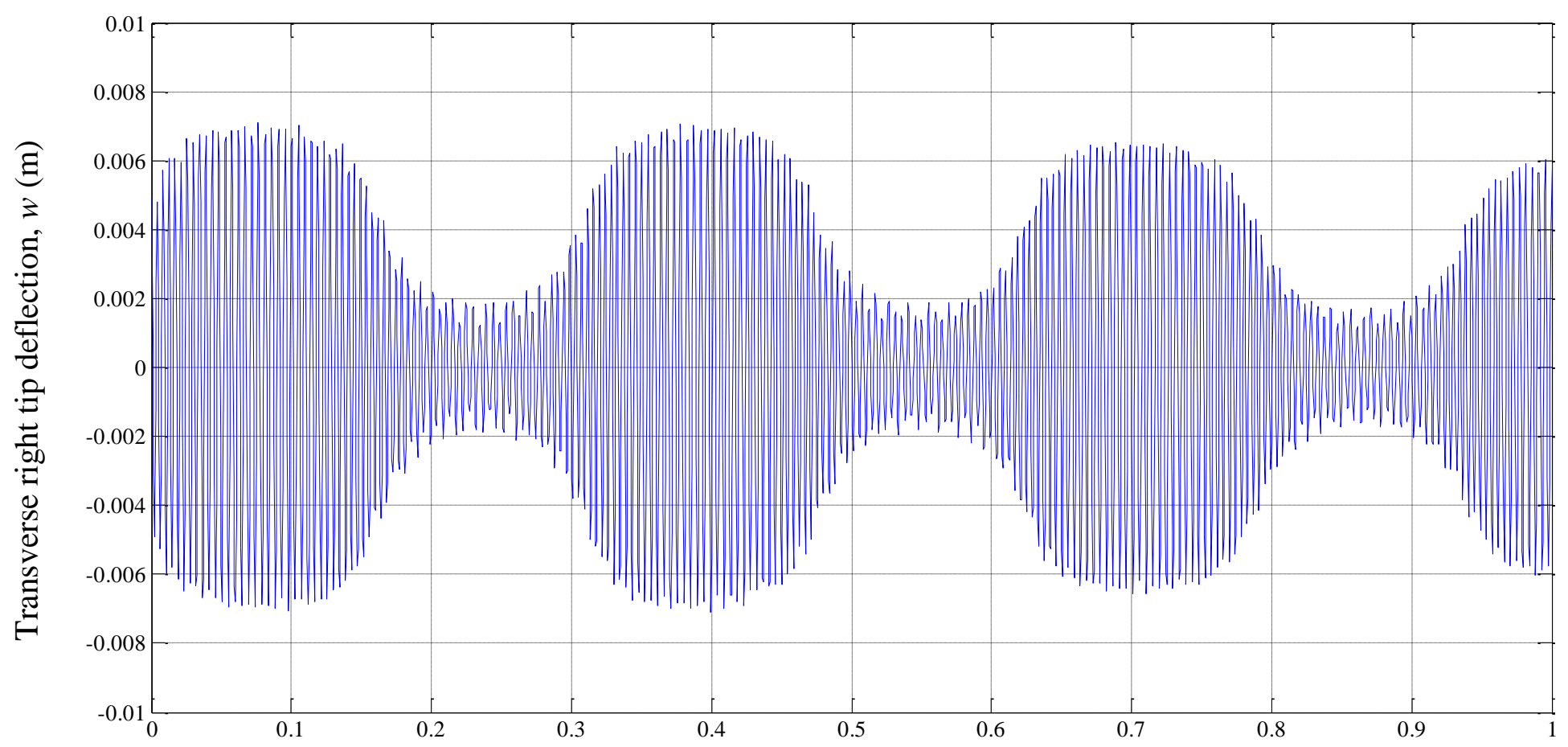

Time (s)

Figure 6.68 Transverse tip deflection at the right end, $w$, HSDT, $\Omega=20 \mathrm{rad} / \mathrm{s},[0 / \pm 45]$ 


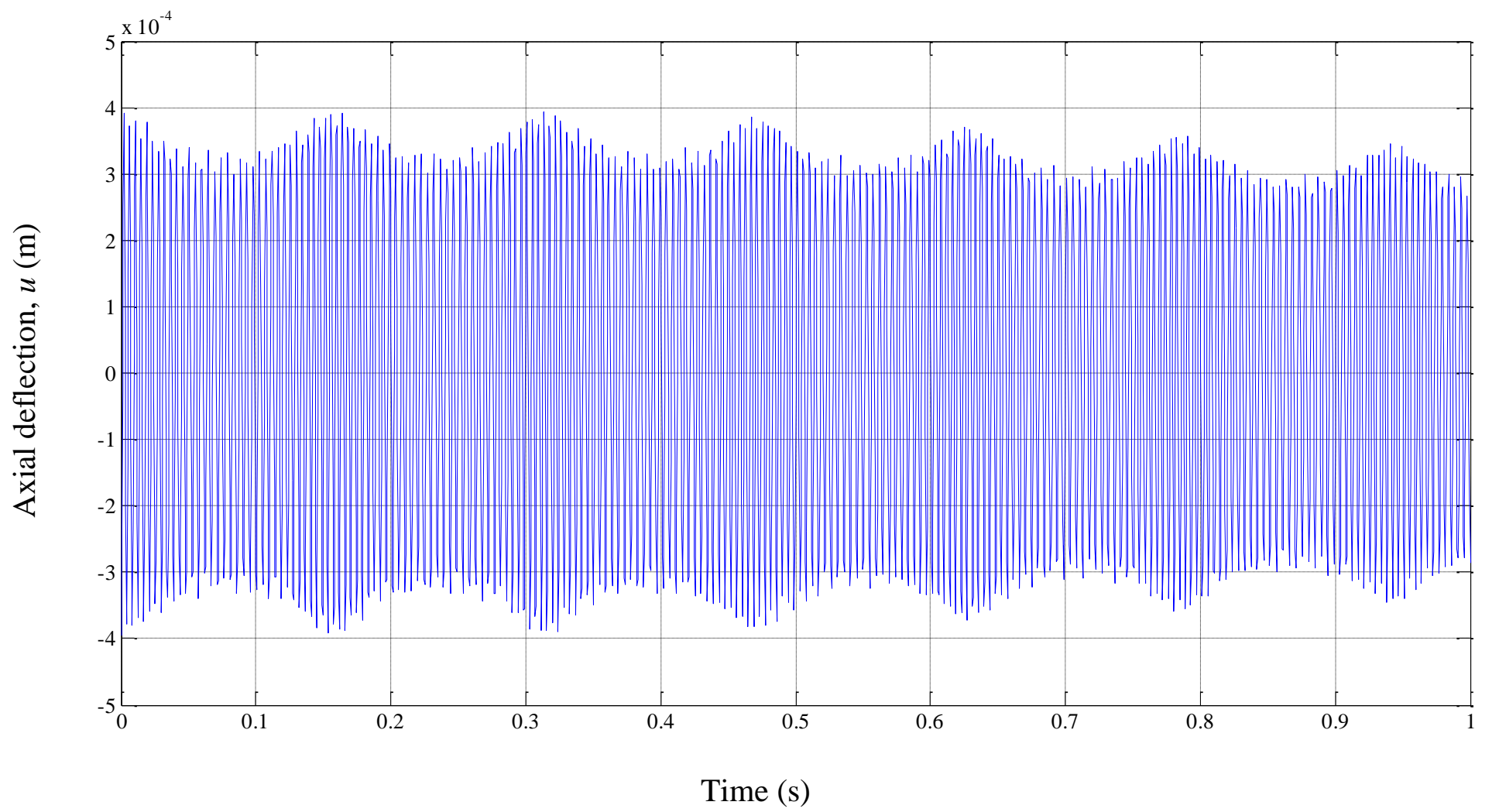

Figure 6.69 Axial deflection at the right end, $u$, HSDT, $\Omega=20 \mathrm{rad} / \mathrm{s},[0 / \pm 45]$ 
Table 6.19 Transverse tip deflection at the left end, $w$, HSDT, $\Omega=20 \mathrm{rad} / \mathrm{s},[0 / \pm 45]$

\begin{tabular}{|c|c|c|c|c|c|c|c|}
\hline$t(\mathrm{~s})$ & $w(\mathrm{~m})$ & $t(\mathrm{~s})$ & $w(\mathrm{~m})$ & $t(\mathrm{~s})$ & $w(\mathrm{~m})$ & $t(\mathrm{~s})$ & $w(\mathrm{~m})$ \\
\hline 0.000000 & 0.005000 & 0.256000 & -0.006984 & 0.503500 & 0.006613 & 0.754500 & 0.002420 \\
\hline 0.010500 & -0.003607 & 0.263000 & 0.006987 & 0.512500 & 0.006726 & 0.768000 & 0.003047 \\
\hline 0.021000 & 0.003198 & 0.274500 & -0.006807 & 0.524000 & -0.006718 & 0.778500 & -0.003990 \\
\hline 0.030000 & 0.002772 & 0.283500 & -0.006724 & 0.531000 & 0.006740 & 0.789000 & 0.004659 \\
\hline 0.041000 & -0.002358 & 0.290000 & 0.006443 & 0.543000 & -0.006824 & 0.799500 & -0.005672 \\
\hline 0.050500 & -0.002130 & 0.303000 & 0.005896 & 0.557500 & -0.006784 & 0.808000 & -0.005789 \\
\hline 0.064500 & -0.001977 & 0.311500 & 0.005168 & 0.564500 & 0.006753 & 0.812500 & -0.006035 \\
\hline 0.079000 & -0.001960 & 0.322000 & -0.004303 & 0.574000 & 0.006722 & 0.821500 & -0.006129 \\
\hline 0.086000 & 0.001915 & 0.337000 & 0.003123 & 0.581000 & -0.006692 & 0.837500 & 0.006286 \\
\hline 0.093500 & -0.001944 & 0.341000 & 0.002630 & 0.592500 & 0.006421 & 0.847000 & 0.006292 \\
\hline 0.109500 & 0.002211 & 0.350000 & 0.002539 & 0.601500 & 0.006230 & 0.854000 & -0.006343 \\
\hline 0.119000 & 0.002437 & 0.366500 & -0.002030 & 0.610000 & 0.006037 & 0.868500 & -0.006313 \\
\hline 0.128000 & 0.002743 & 0.375500 & -0.001989 & 0.625000 & -0.005133 & 0.875500 & 0.006250 \\
\hline 0.139000 & -0.003113 & 0.382500 & 0.001934 & 0.631000 & 0.004442 & 0.880500 & 0.006182 \\
\hline 0.143000 & -0.003820 & 0.397000 & 0.001947 & 0.644000 & 0.003437 & 0.899000 & 0.006110 \\
\hline 0.157500 & 0.005008 & 0.404000 & -0.001924 & 0.657000 & 0.002660 & 0.906000 & -0.006043 \\
\hline 0.166000 & 0.005499 & 0.418500 & -0.002164 & 0.666000 & 0.002306 & 0.917000 & 0.005861 \\
\hline 0.172500 & -0.006344 & 0.427500 & -0.002262 & 0.675000 & 0.002092 & 0.921500 & 0.005581 \\
\hline 0.185500 & -0.006750 & 0.437000 & -0.002472 & 0.684500 & 0.001839 & 0.930000 & 0.005435 \\
\hline 0.194500 & -0.006845 & 0.448000 & 0.003051 & 0.691500 & -0.001887 & 0.940500 & -0.004380 \\
\hline 0.206000 & 0.006922 & 0.458500 & -0.003631 & 0.706000 & -0.001718 & 0.951000 & 0.003665 \\
\hline 0.213000 & -0.007031 & 0.469000 & 0.004322 & 0.713000 & 0.001831 & 0.961500 & -0.002833 \\
\hline 0.220000 & 0.007069 & 0.479500 & -0.005735 & 0.720000 & -0.001839 & 0.975000 & -0.002230 \\
\hline 0.234500 & 0.007045 & 0.488000 & -0.005899 & 0.736500 & 0.002027 & 0.984000 & -0.002028 \\
\hline 0.241500 & -0.007055 & 0.492500 & -0.006393 & 0.743500 & -0.002135 & 0.993000 & -0.001877 \\
\hline
\end{tabular}




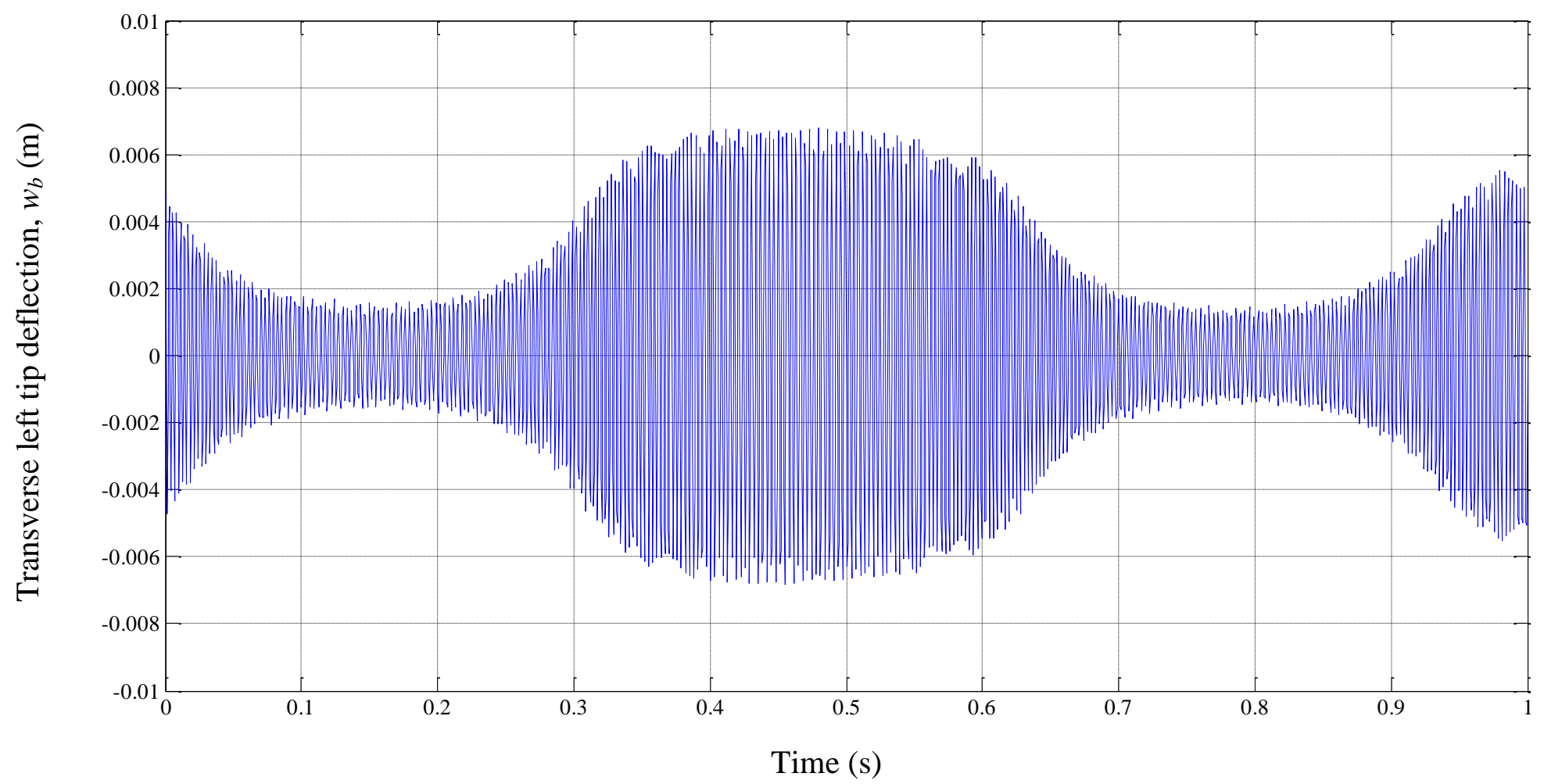

Figure 6.70 Transverse tip deflection at the left end, $w_{b}$, HSDT, $\Omega=10 \mathrm{rad} / \mathrm{s},[0 / \pm 20]$ 


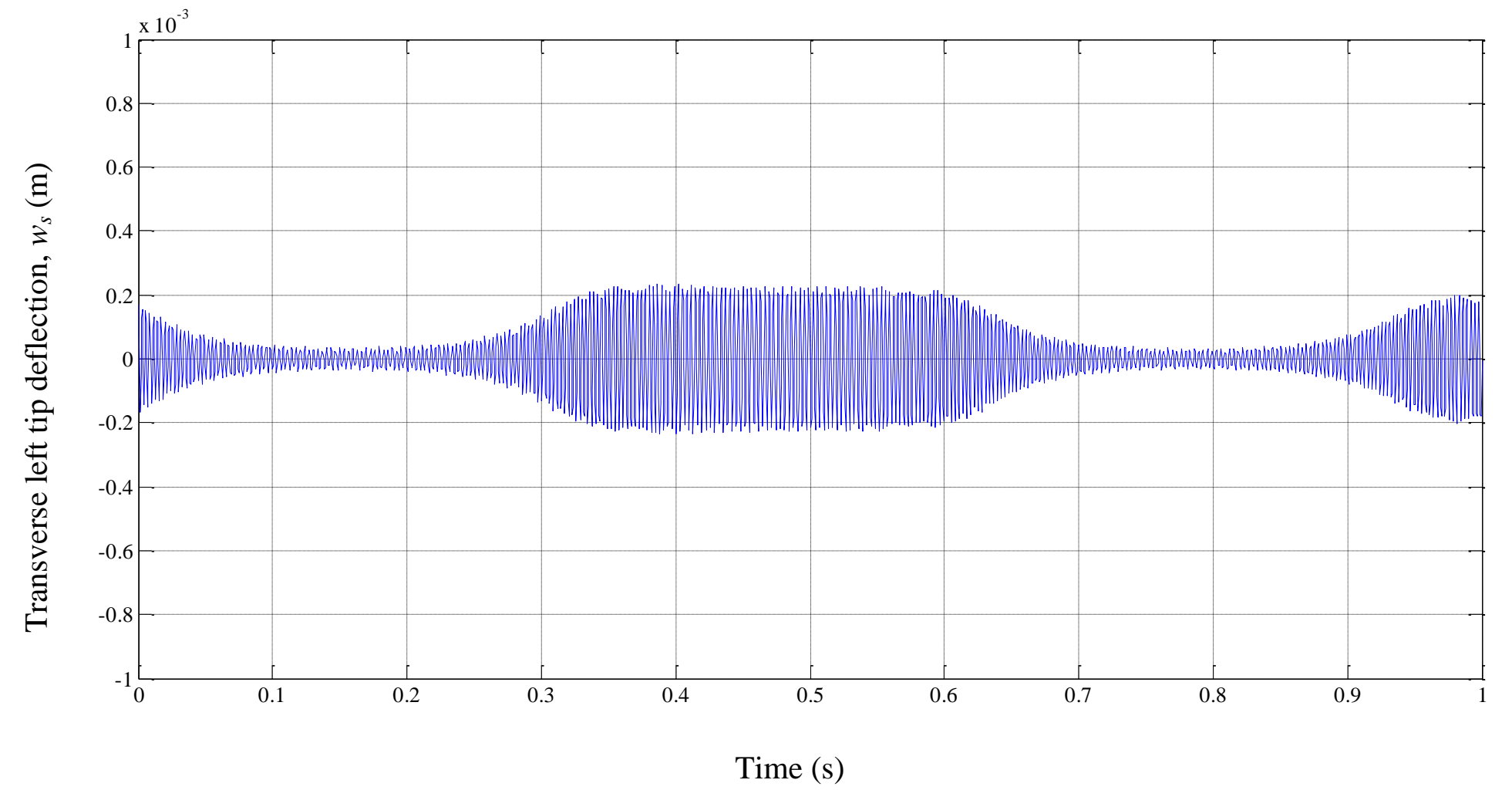

Figure 6.71 Transverse tip deflection at the left end, $w_{s}$, HSDT, $\Omega=10 \mathrm{rad} / \mathrm{s},[0 / \pm 20]$ 


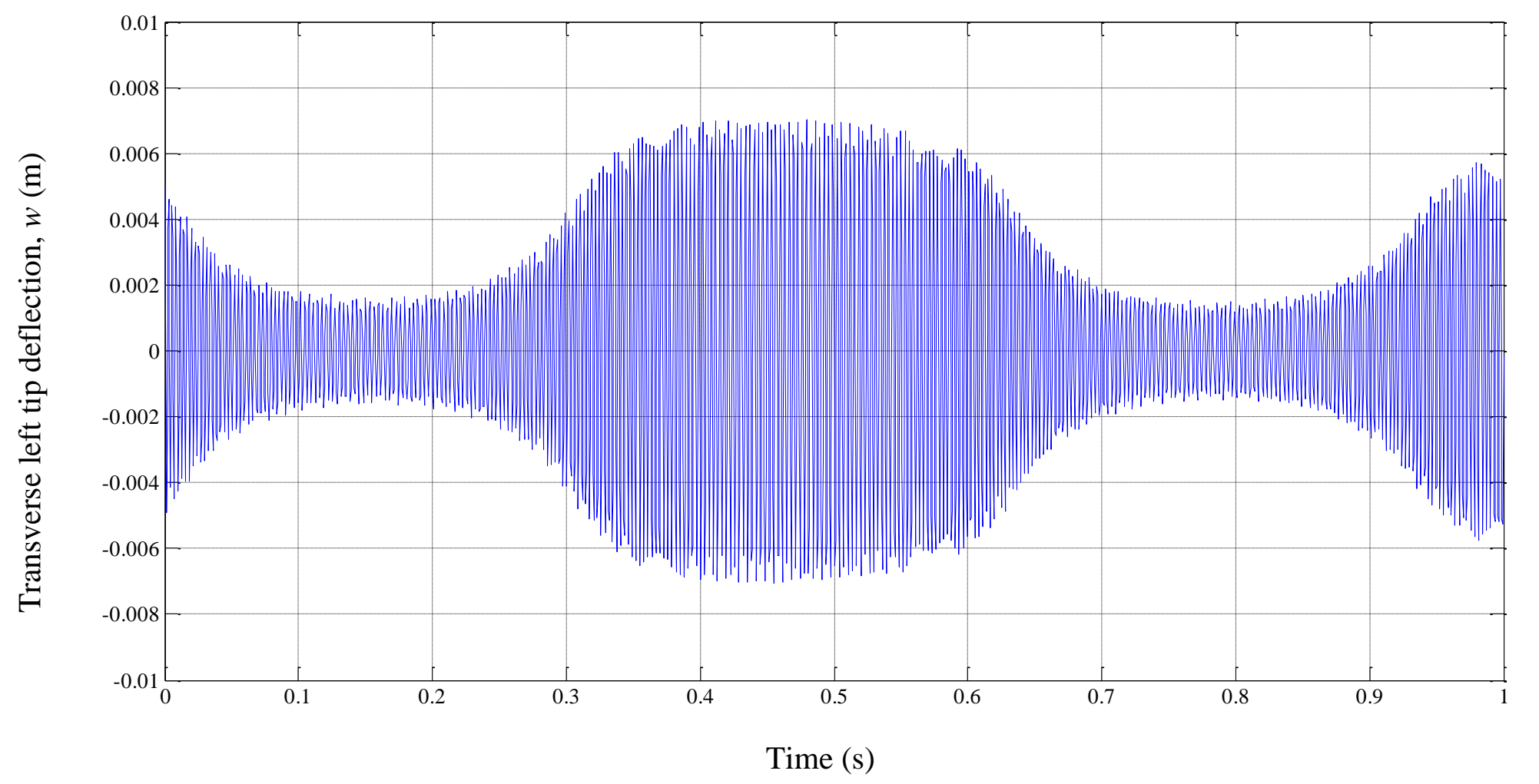

Figure 6.72 Transverse tip deflection at the left end, $w$, HSDT, $\Omega=10 \mathrm{rad} / \mathrm{s},[0 / \pm 20]$ 


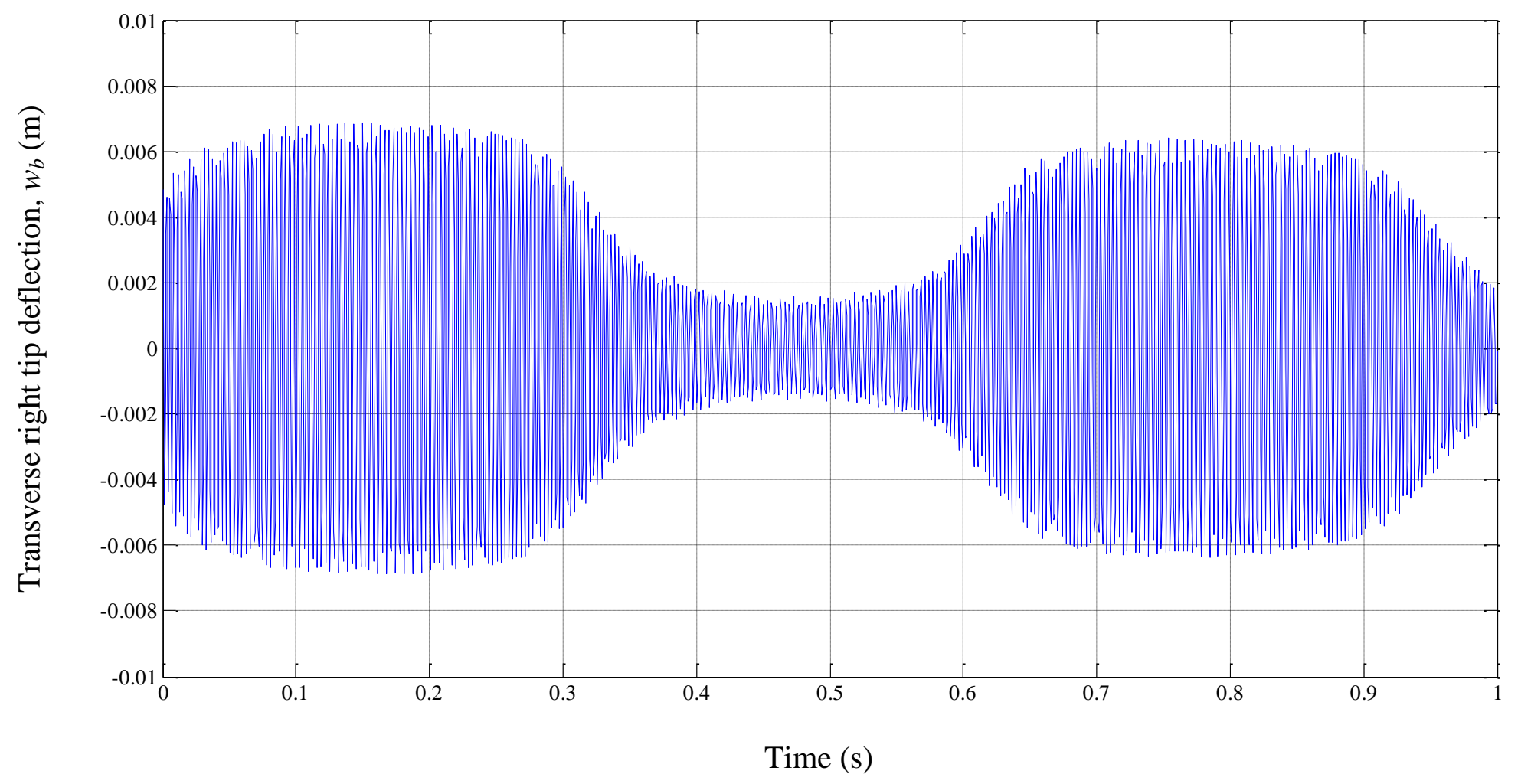

Figure 6.73 Transverse tip deflection at the right end, $w_{b}, \mathrm{HSDT}, \Omega=10 \mathrm{rad} / \mathrm{s},[0 / \pm 20]$ 


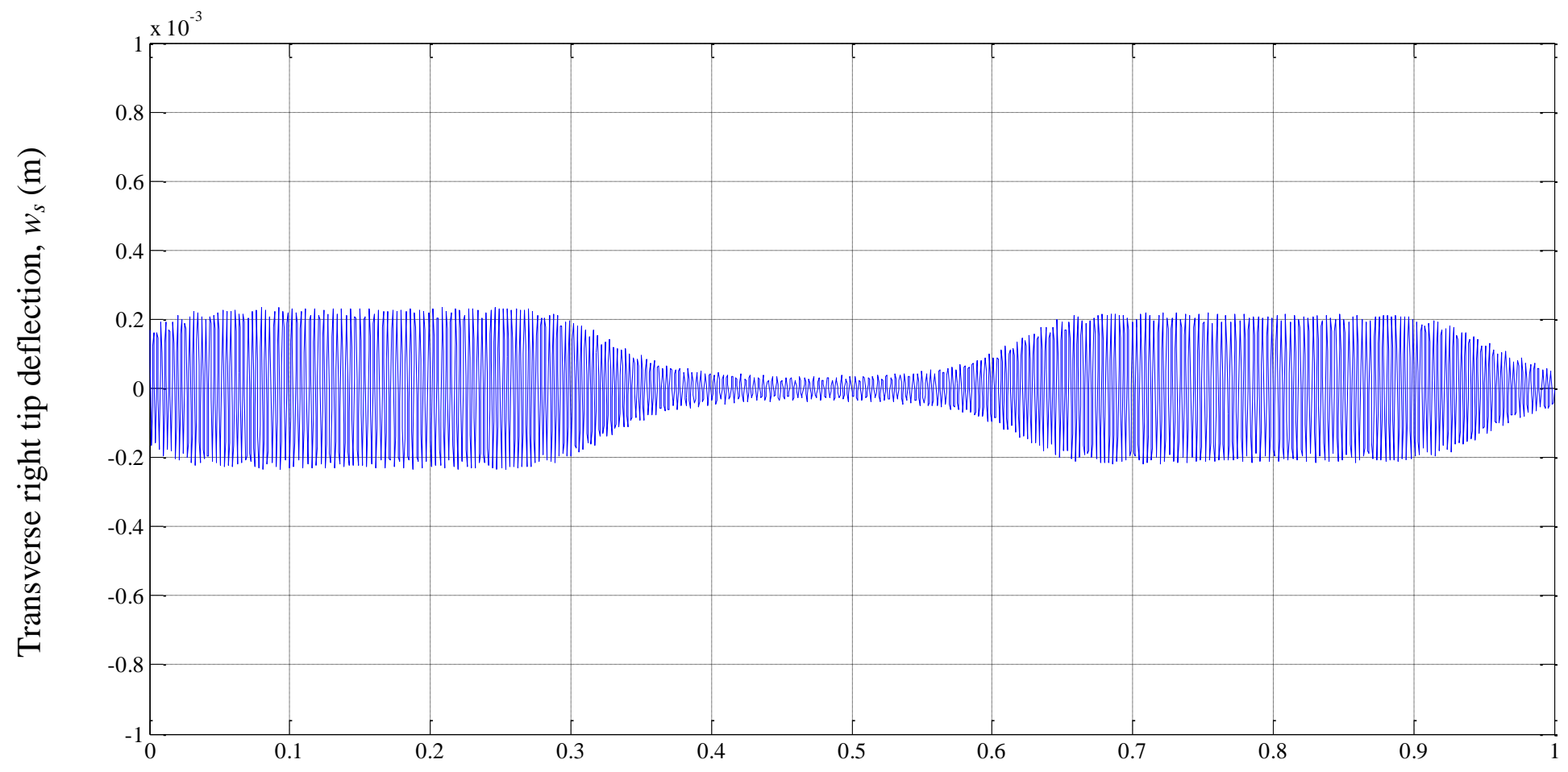

Time (s)

Figure 6.74 Transverse tip deflection at the right end, $w_{s}$, HSDT, $\Omega=10 \mathrm{rad} / \mathrm{s},[0 / \pm 20]$ 


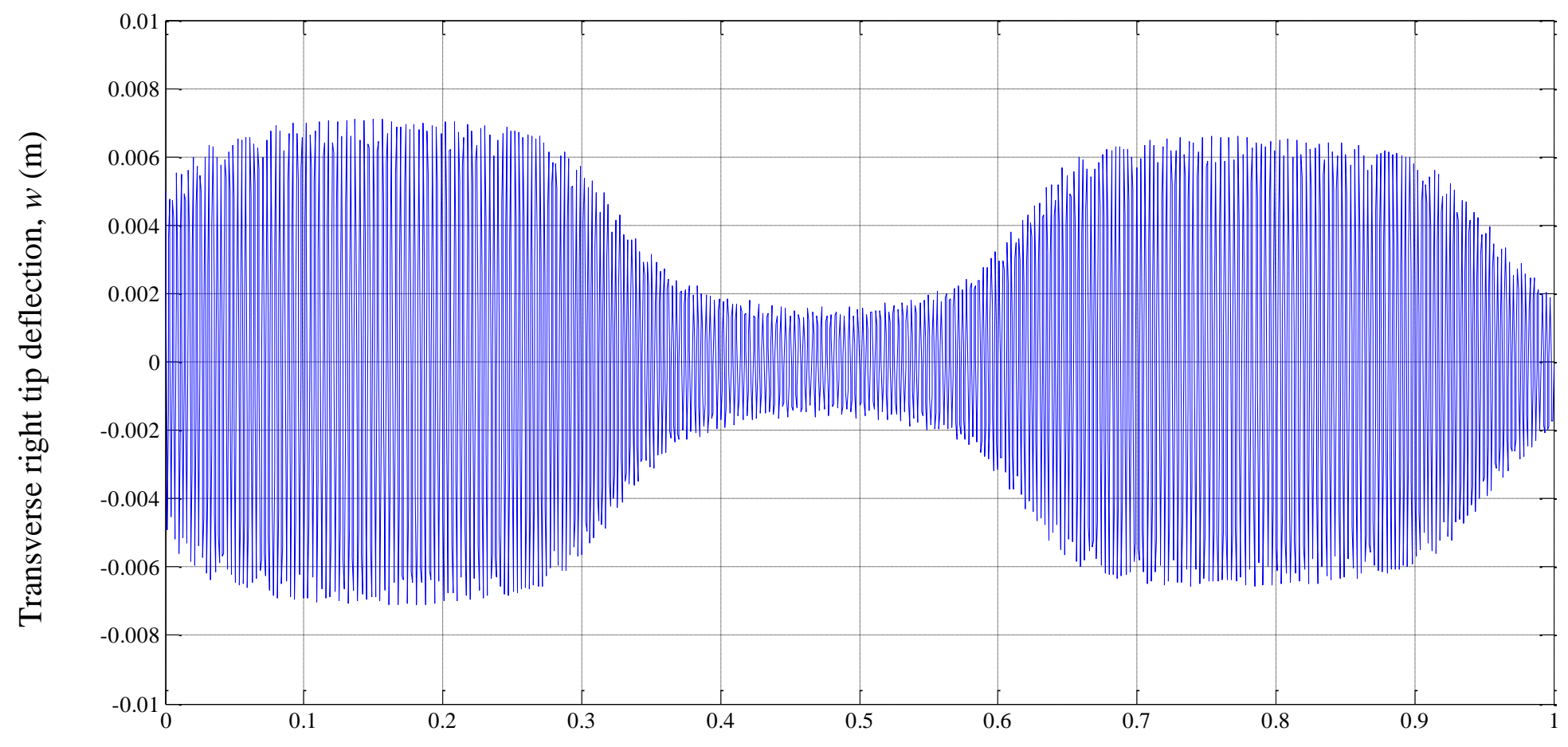

Time (s)

Figure 6.75 Transverse tip deflection at the right end, $w$, HSDT, $\Omega=10 \mathrm{rad} / \mathrm{s},[0 / \pm 20]$ 


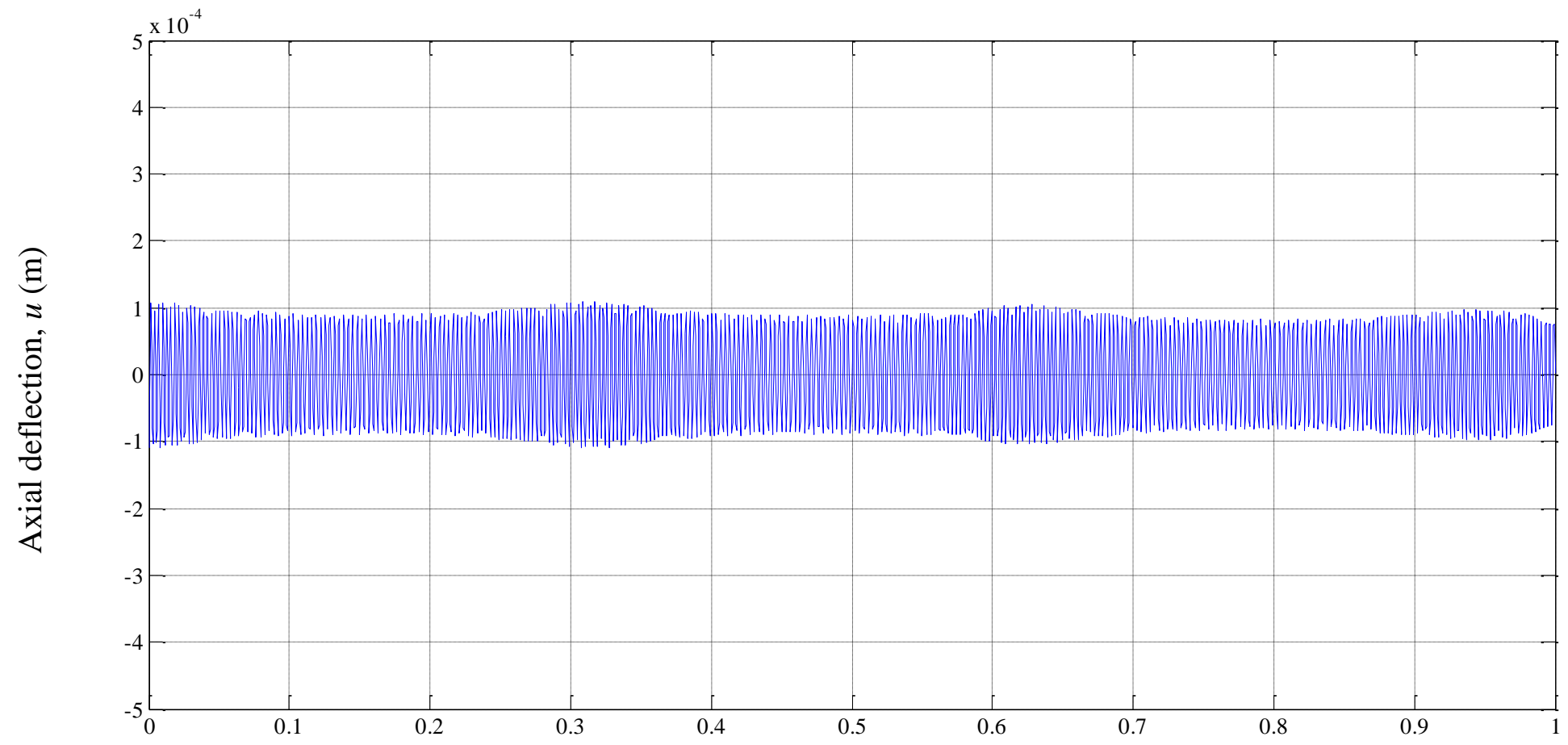

Time (s)

Figure 6.76 Axial deflection at the right end, $u$, HSDT, $\Omega=10 \mathrm{rad} / \mathrm{s},[0 / \pm 20]$ 
Table 6.20 Transverse tip deflection at the left end, $w$, HSDT, $\Omega=10 \mathrm{rad} / \mathrm{s},[0 / \pm 20]$

\begin{tabular}{|c|c|c|c|c|c|c|c|}
\hline$t(\mathrm{~s})$ & $w(\mathrm{~m})$ & $t(\mathrm{~s})$ & $w(\mathrm{~m})$ & $t(\mathrm{~s})$ & $w(\mathrm{~m})$ & $t(\mathrm{~s})$ & $w(\mathrm{~m})$ \\
\hline 0.000000 & 0.005000 & 0.258000 & 0.002522 & 0.505500 & 0.006929 & 0.750500 & 0.001446 \\
\hline 0.010000 & -0.004245 & 0.267000 & 0.002777 & 0.512000 & 0.006920 & 0.766500 & 0.001536 \\
\hline 0.020000 & 0.003715 & 0.274500 & -0.003008 & 0.528000 & 0.006868 & 0.771500 & -0.001490 \\
\hline 0.030000 & -0.003338 & 0.287500 & 0.003543 & 0.537500 & 0.006772 & 0.786000 & 0.001463 \\
\hline 0.047500 & -0.002680 & 0.299000 & 0.004171 & 0.540500 & 0.006587 & 0.791000 & -0.001503 \\
\hline 0.055000 & 0.002490 & 0.309000 & -0.004809 & 0.551500 & -0.006719 & 0.801000 & -0.001430 \\
\hline 0.064000 & 0.002265 & 0.319000 & 0.005203 & 0.567000 & -0.006060 & 0.817000 & -0.001536 \\
\hline 0.078000 & -0.002111 & 0.327500 & 0.005605 & 0.573000 & -0.006125 & 0.822000 & 0.001557 \\
\hline 0.084000 & -0.001921 & 0.337500 & -0.006086 & 0.589000 & 0.005854 & 0.838000 & 0.001664 \\
\hline 0.090500 & -0.001946 & 0.349000 & -0.006243 & 0.593500 & -0.006174 & 0.849000 & -0.001679 \\
\hline 0.101500 & 0.001815 & 0.355000 & -0.006521 & 0.606500 & 0.005715 & 0.855500 & -0.001745 \\
\hline 0.119000 & -0.001709 & 0.364000 & -0.006256 & 0.616500 & -0.005373 & 0.869500 & 0.001830 \\
\hline 0.124000 & 0.001715 & 0.379000 & -0.006571 & 0.626500 & 0.004922 & 0.877000 & -0.002077 \\
\hline 0.138500 & -0.001551 & 0.388000 & -0.006886 & 0.636500 & -0.004235 & 0.884500 & 0.002243 \\
\hline 0.145000 & -0.001604 & 0.399000 & 0.006787 & 0.643500 & 0.003815 & 0.899500 & 0.002576 \\
\hline 0.150000 & 0.001610 & 0.402000 & 0.006963 & 0.655000 & 0.003268 & 0.909500 & -0.002680 \\
\hline 0.169500 & 0.001618 & 0.413000 & -0.006980 & 0.661000 & 0.003004 & 0.918500 & -0.003105 \\
\hline 0.174500 & -0.001666 & 0.429000 & -0.007027 & 0.674000 & -0.002602 & 0.927000 & -0.003568 \\
\hline 0.189000 & 0.001566 & 0.435500 & -0.007043 & 0.681500 & 0.002451 & 0.937000 & 0.004191 \\
\hline 0.195500 & 0.001690 & 0.448500 & -0.006992 & 0.690500 & 0.002221 & 0.947000 & -0.004676 \\
\hline 0.200500 & -0.001756 & 0.455000 & -0.007050 & 0.704500 & -0.001907 & 0.955500 & -0.004983 \\
\hline 0.218000 & 0.001845 & 0.461500 & -0.007027 & 0.710500 & -0.001913 & 0.965500 & 0.005323 \\
\hline 0.229000 & -0.002025 & 0.479500 & 0.007017 & 0.723000 & -0.001758 & 0.978500 & -0.005656 \\
\hline 0.230500 & 0.001964 & 0.486000 & 0.006987 & 0.739000 & -0.001553 & 0.981500 & -0.005747 \\
\hline 0.249000 & 0.002309 & 0.491000 & -0.006932 & 0.740500 & 0.001621 & 0.990500 & -0.005371 \\
\hline
\end{tabular}




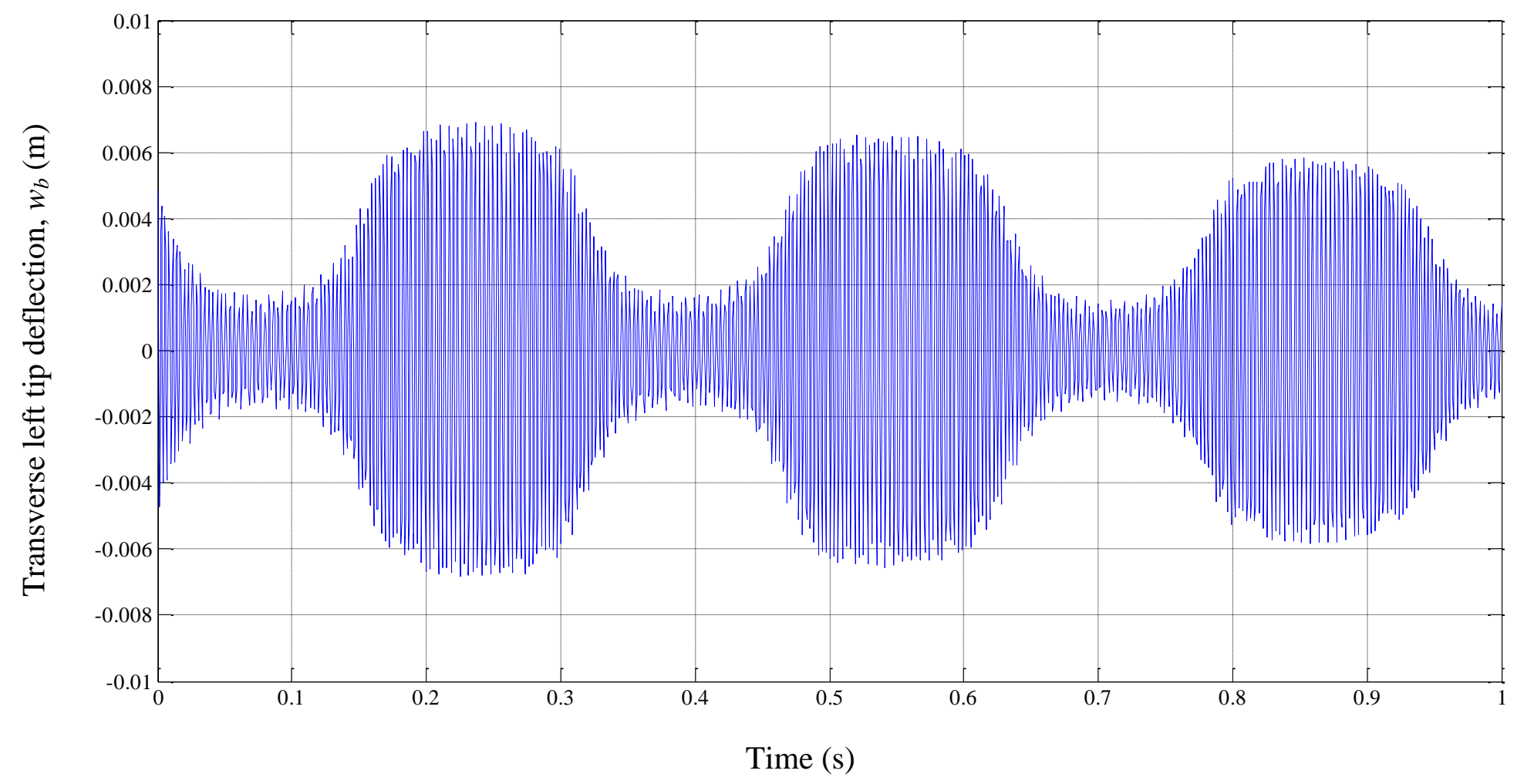

Figure 6.77 Transverse tip deflection at the left end, $w_{b}$, HSDT, $\Omega=20 \mathrm{rad} / \mathrm{s},[0 / \pm 20]$ 


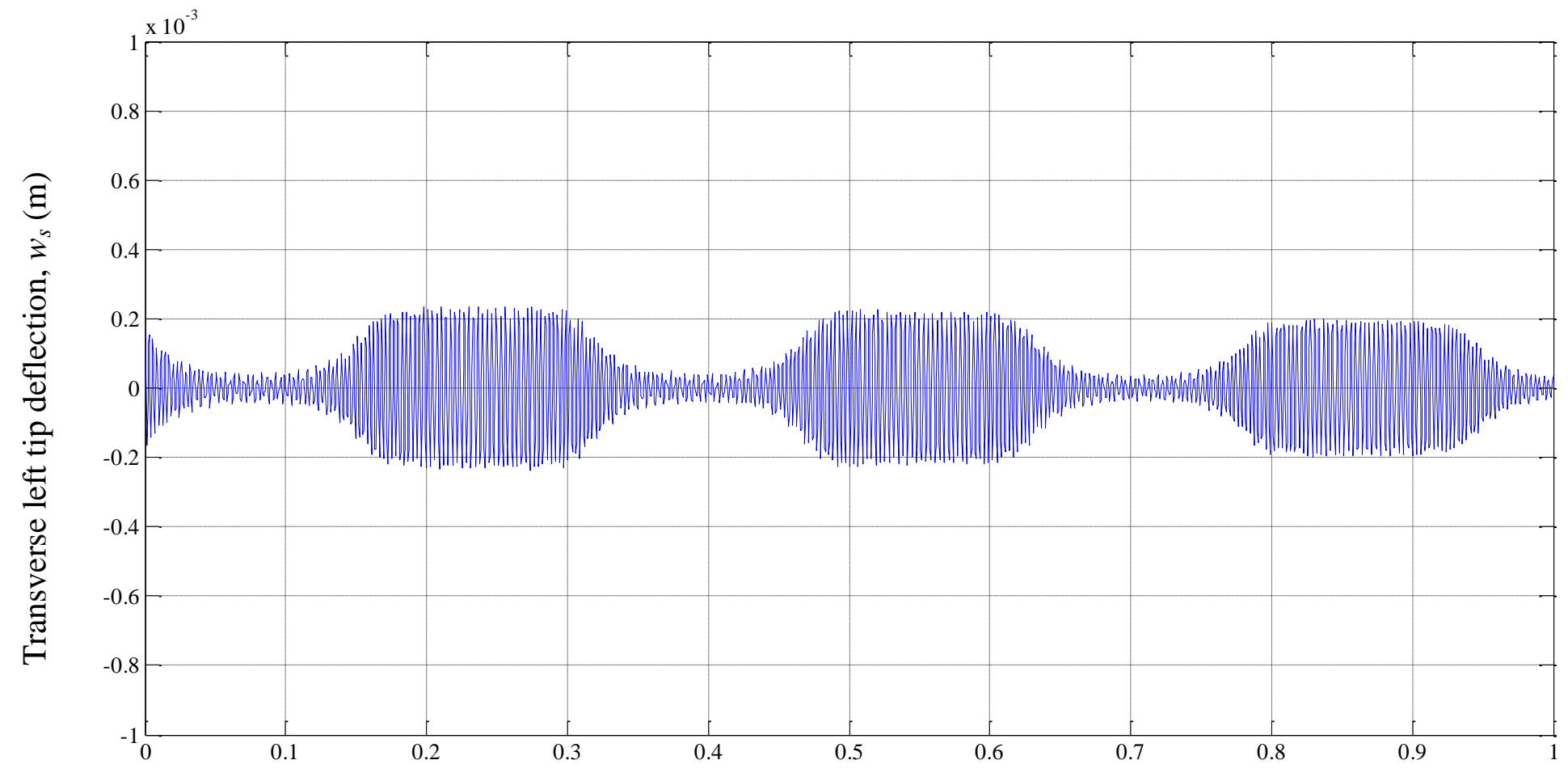

Time (s)

Figure 6.78 Transverse tip deflection at the left end, $w_{s}$, HSDT, $\Omega=20 \mathrm{rad} / \mathrm{s},[0 / \pm 20]$ 


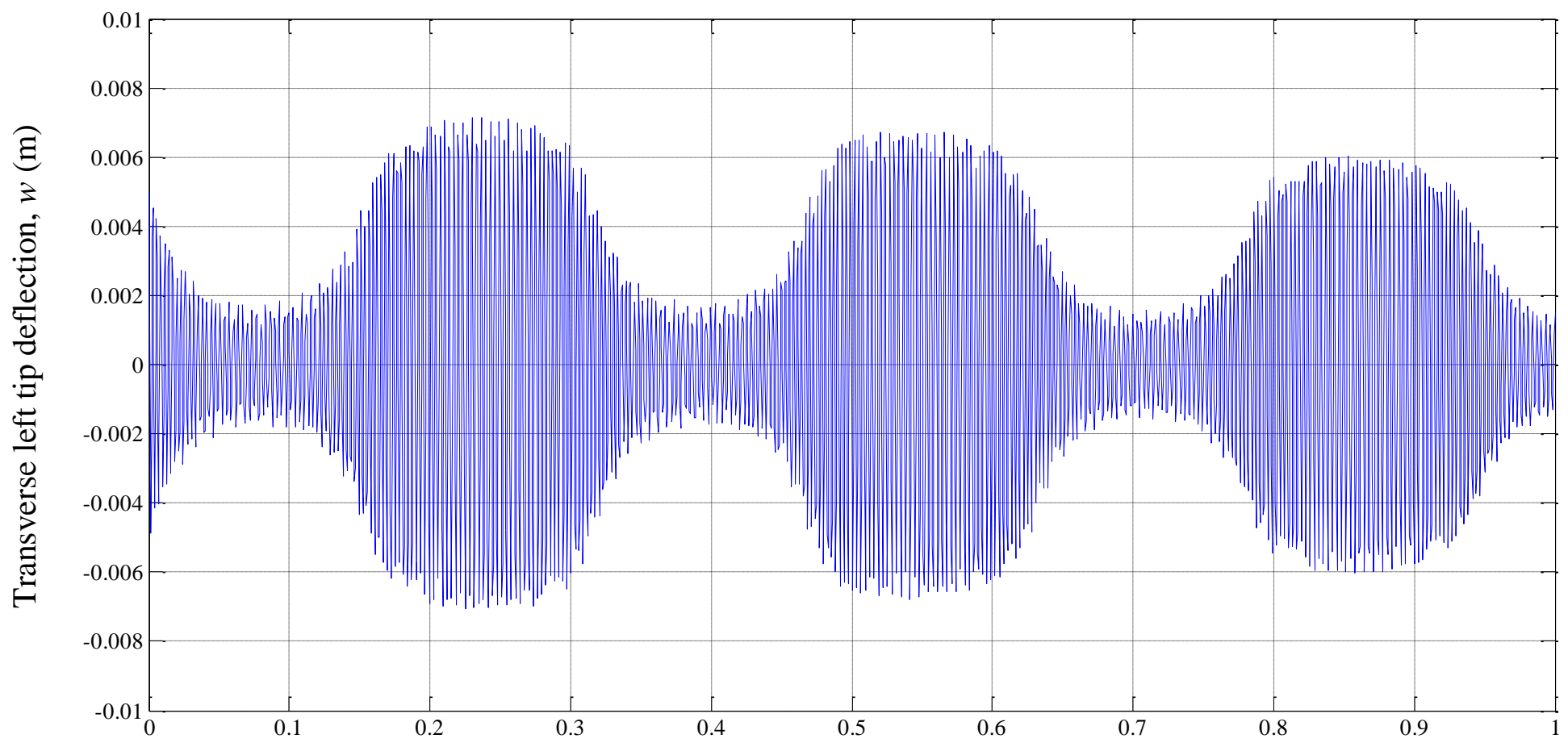

Time (s)

Figure 6.79 Transverse tip deflection at the left end, $w$, HSDT, $\Omega=20 \mathrm{rad} / \mathrm{s},[0 / \pm 20]$ 


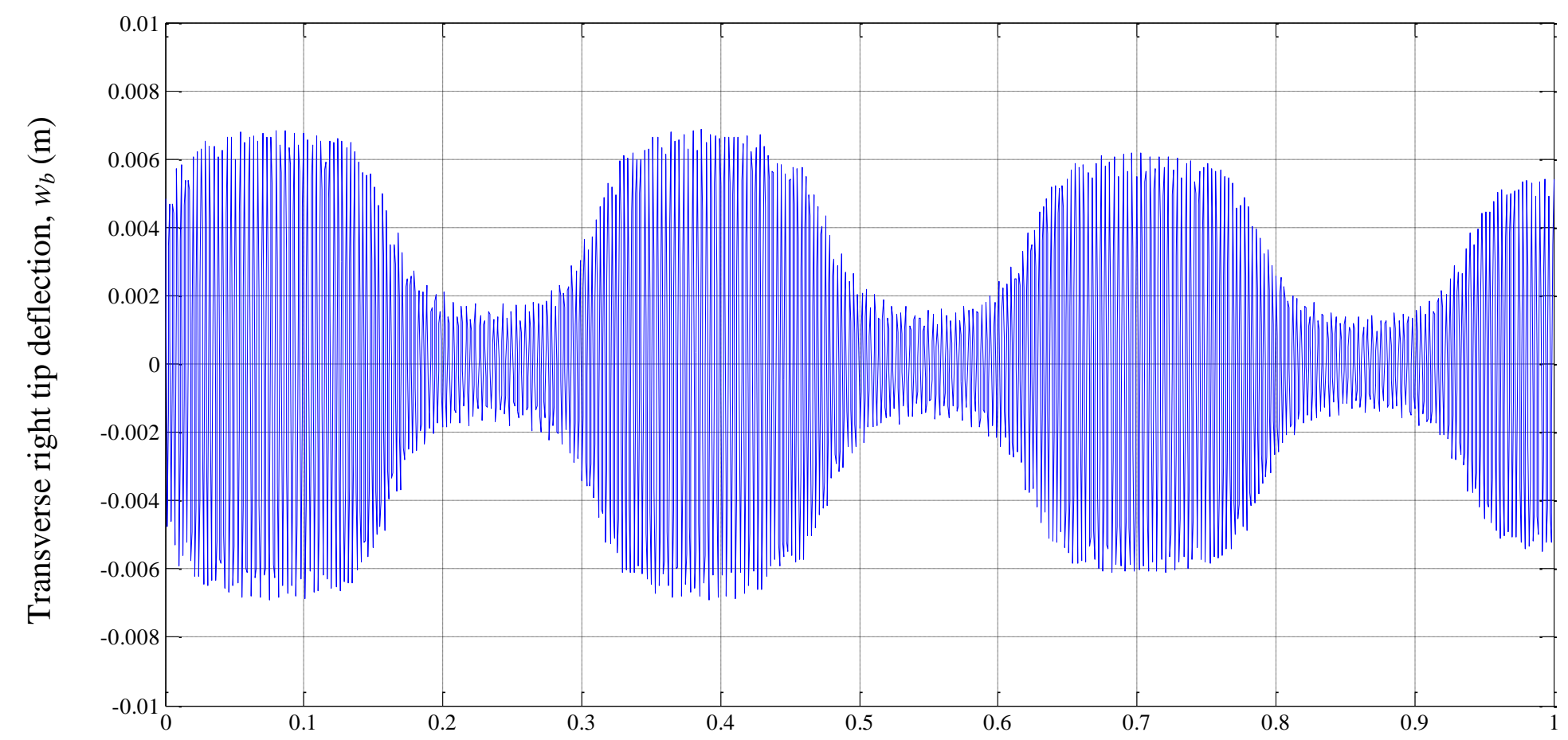

Time (s)

Figure 6.80 Transverse tip deflection at the right end, $w_{b}$, HSDT, $\Omega=20 \mathrm{rad} / \mathrm{s},[0 / \pm 20]$ 


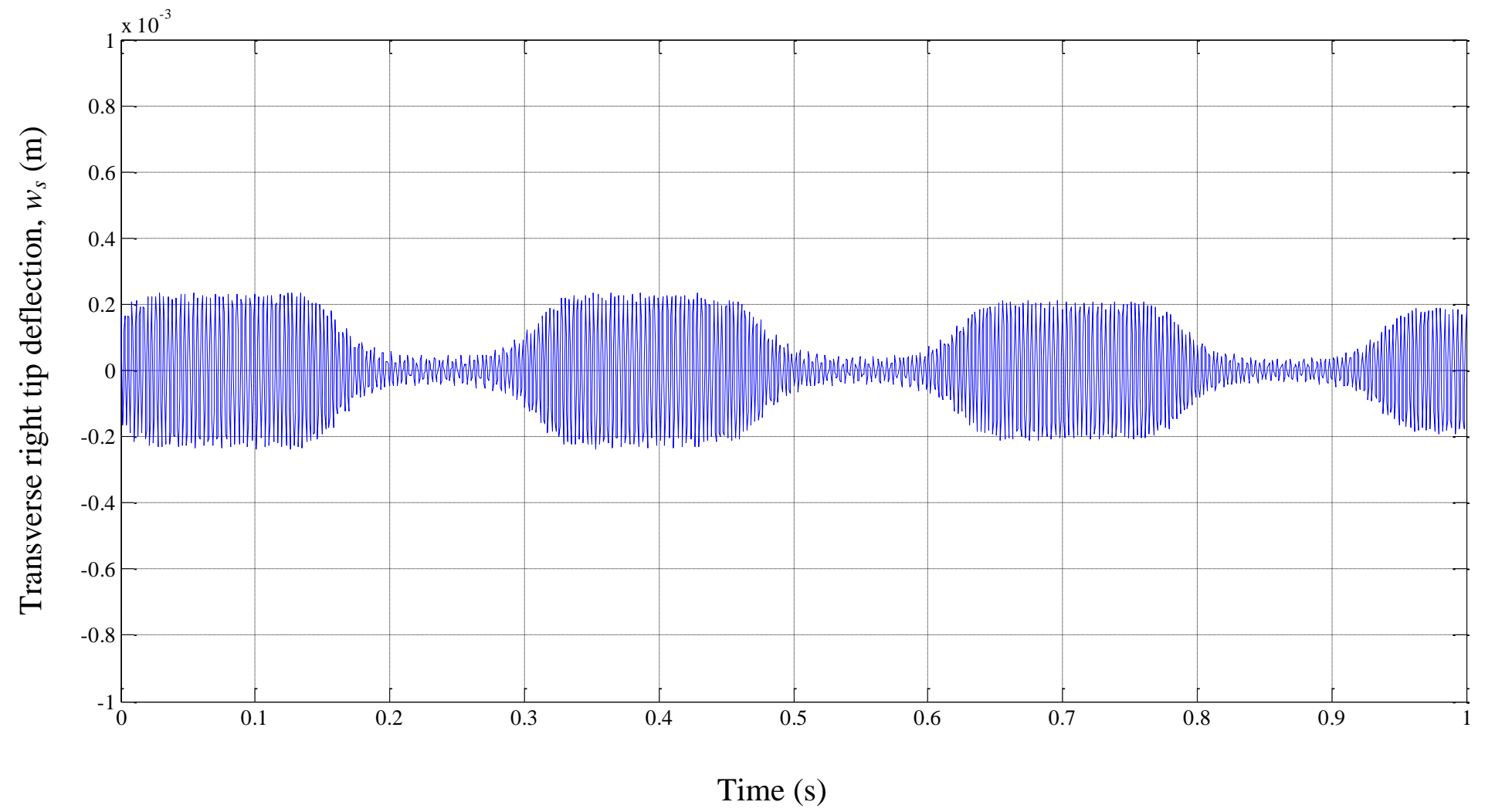

Figure 6.81 Transverse tip deflection at the right end, $w_{s}, \mathrm{HSDT}, \Omega=20 \mathrm{rad} / \mathrm{s},[0 / \pm 20]$ 


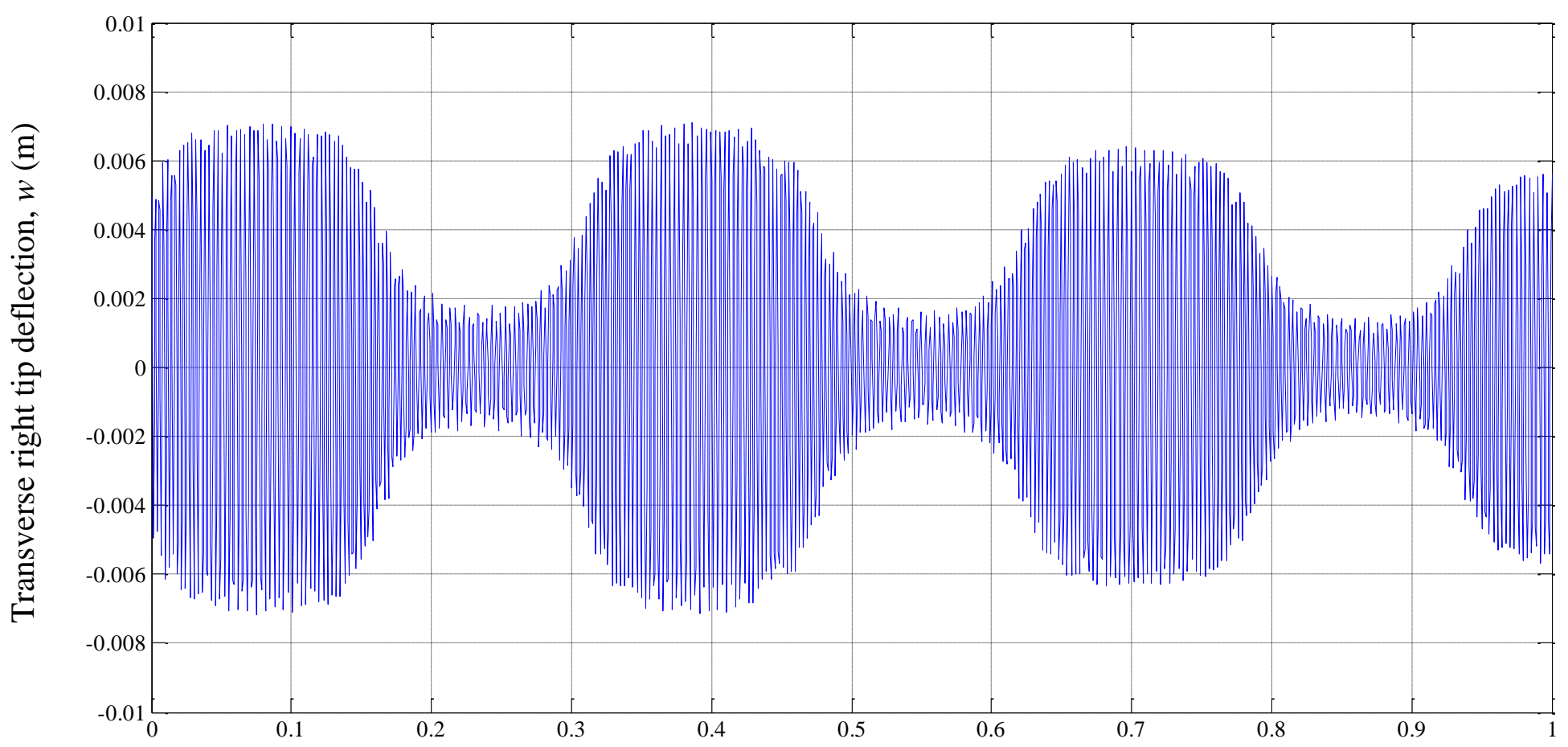

Time (s)

Figure 6.82 Transverse tip deflection at the right end, $w$, HSDT, $\Omega=20 \mathrm{rad} / \mathrm{s},[0 / \pm 20]$ 


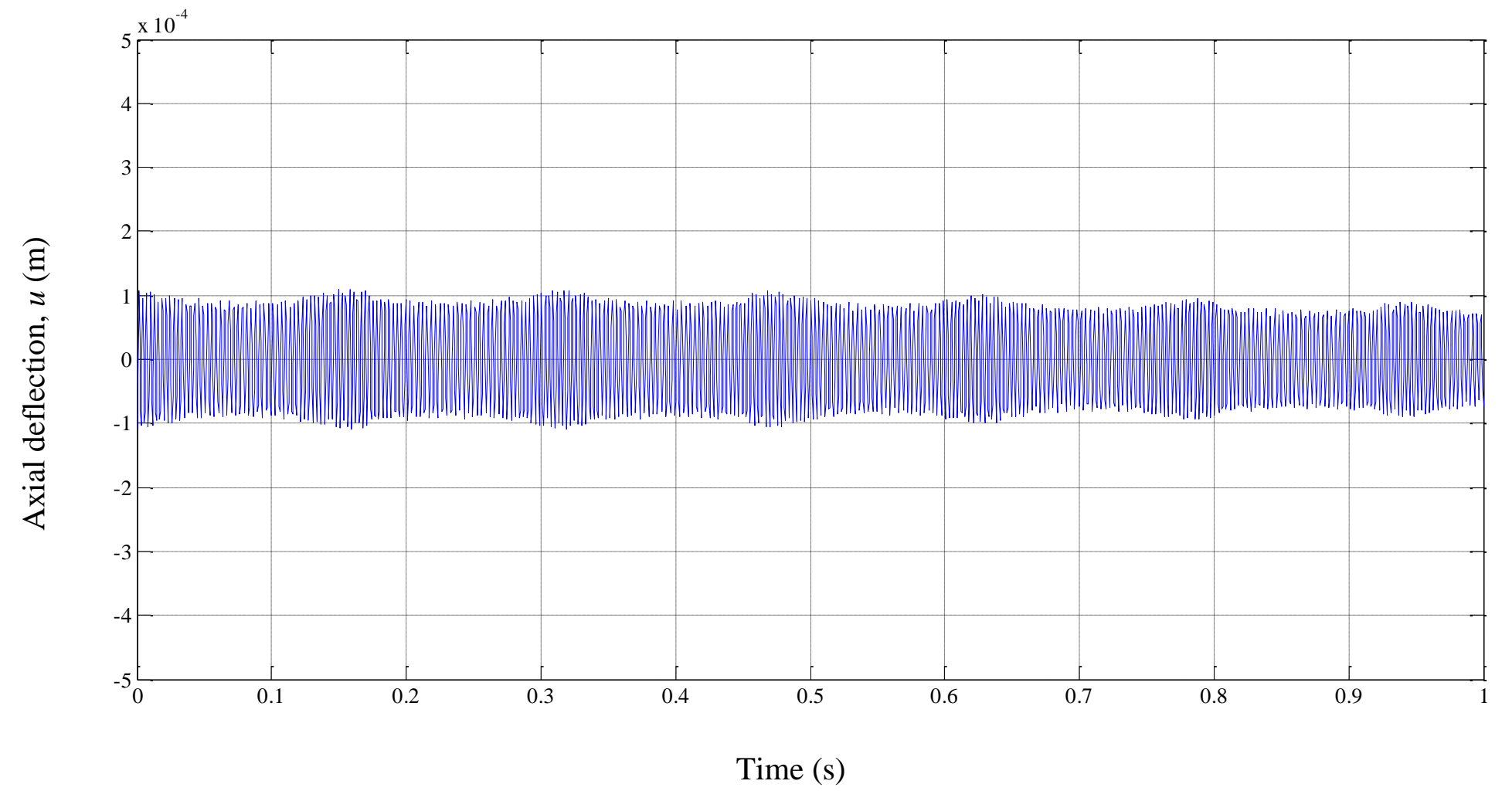

Figure 6.83 Axial deflection at the right end, $u, \mathrm{HSDT}, \Omega=20 \mathrm{rad} / \mathrm{s},[0 / \pm 20]$ 
Table 6.21 Transverse tip deflection at the left end, $w$, HSDT, $\Omega=20 \mathrm{rad} / \mathrm{s},[0 / \pm 20]$

\begin{tabular}{|c|c|c|c|c|c|c|c|}
\hline$t(\mathrm{~s})$ & $w(\mathrm{~m})$ & $t(\mathrm{~s})$ & $w(\mathrm{~m})$ & $t(\mathrm{~s})$ & $w(\mathrm{~m})$ & $t(\mathrm{~s})$ & $w(\mathrm{~m})$ \\
\hline 0.000000 & 0.005000 & 0.256000 & 0.007112 & 0.508000 & 0.006652 & 0.755500 & -0.002253 \\
\hline 0.010000 & -0.003523 & 0.262500 & 0.006988 & 0.519000 & -0.006689 & 0.764500 & -0.002705 \\
\hline 0.024500 & -0.002877 & 0.273500 & -0.006975 & 0.520500 & 0.006735 & 0.779000 & -0.003531 \\
\hline 0.032000 & 0.002429 & 0.281000 & 0.006571 & 0.535000 & -0.006724 & 0.789000 & 0.004737 \\
\hline 0.046000 & -0.002114 & 0.297500 & -0.006483 & 0.541500 & -0.006799 & 0.799000 & -0.005464 \\
\hline 0.057000 & 0.001808 & 0.300500 & -0.006035 & 0.553000 & 0.006680 & 0.800500 & 0.005395 \\
\hline 0.062000 & -0.001803 & 0.310500 & 0.005492 & 0.566000 & 0.006705 & 0.816500 & -0.005342 \\
\hline 0.072000 & -0.001601 & 0.320500 & -0.004371 & 0.572500 & 0.006628 & 0.829000 & -0.005934 \\
\hline 0.088000 & -0.001815 & 0.332000 & -0.003316 & 0.583500 & -0.006544 & 0.837000 & 0.005981 \\
\hline 0.093000 & 0.001850 & 0.341000 & -0.002575 & 0.597500 & 0.006334 & 0.846500 & 0.005992 \\
\hline 0.109000 & 0.002062 & 0.350000 & -0.002253 & 0.603500 & 0.006168 & 0.858000 & -0.006037 \\
\hline 0.115500 & 0.001983 & 0.362500 & -0.001951 & 0.616500 & -0.005610 & 0.864500 & -0.005979 \\
\hline 0.129000 & -0.002627 & 0.373500 & 0.001898 & 0.621000 & 0.005028 & 0.877500 & -0.005983 \\
\hline 0.139500 & 0.003276 & 0.383500 & 0.001706 & 0.631000 & -0.003992 & 0.884000 & -0.005931 \\
\hline 0.149500 & -0.004339 & 0.398000 & -0.001731 & 0.641000 & 0.003268 & 0.892000 & 0.005849 \\
\hline 0.159500 & 0.005264 & 0.403000 & 0.001750 & 0.651000 & -0.002660 & 0.901500 & 0.005766 \\
\hline 0.169500 & -0.005964 & 0.419000 & 0.001899 & 0.660000 & -0.002146 & 0.910500 & 0.005159 \\
\hline 0.172500 & -0.006182 & 0.425500 & 0.002021 & 0.672500 & -0.001899 & 0.924000 & -0.005286 \\
\hline 0.184000 & -0.006393 & 0.438000 & 0.002196 & 0.683500 & 0.001692 & 0.930000 & -0.004948 \\
\hline 0.199500 & -0.006913 & 0.447000 & 0.002598 & 0.690000 & 0.001569 & 0.940000 & 0.004119 \\
\hline 0.209000 & -0.006996 & 0.458500 & 0.003561 & 0.709500 & 0.001584 & 0.950000 & -0.003093 \\
\hline 0.210500 & 0.007078 & 0.468500 & -0.004776 & 0.714500 & -0.001588 & 0.960000 & 0.002555 \\
\hline 0.225000 & -0.007069 & 0.478500 & 0.005622 & 0.729000 & 0.001501 & 0.975000 & 0.001916 \\
\hline 0.236500 & 0.007139 & 0.488500 & -0.006002 & 0.735500 & 0.001751 & 0.982500 & -0.001769 \\
\hline 0.249500 & 0.007031 & 0.499000 & 0.006455 & 0.748000 & 0.002014 & 0.995000 & -0.001488 \\
\hline
\end{tabular}




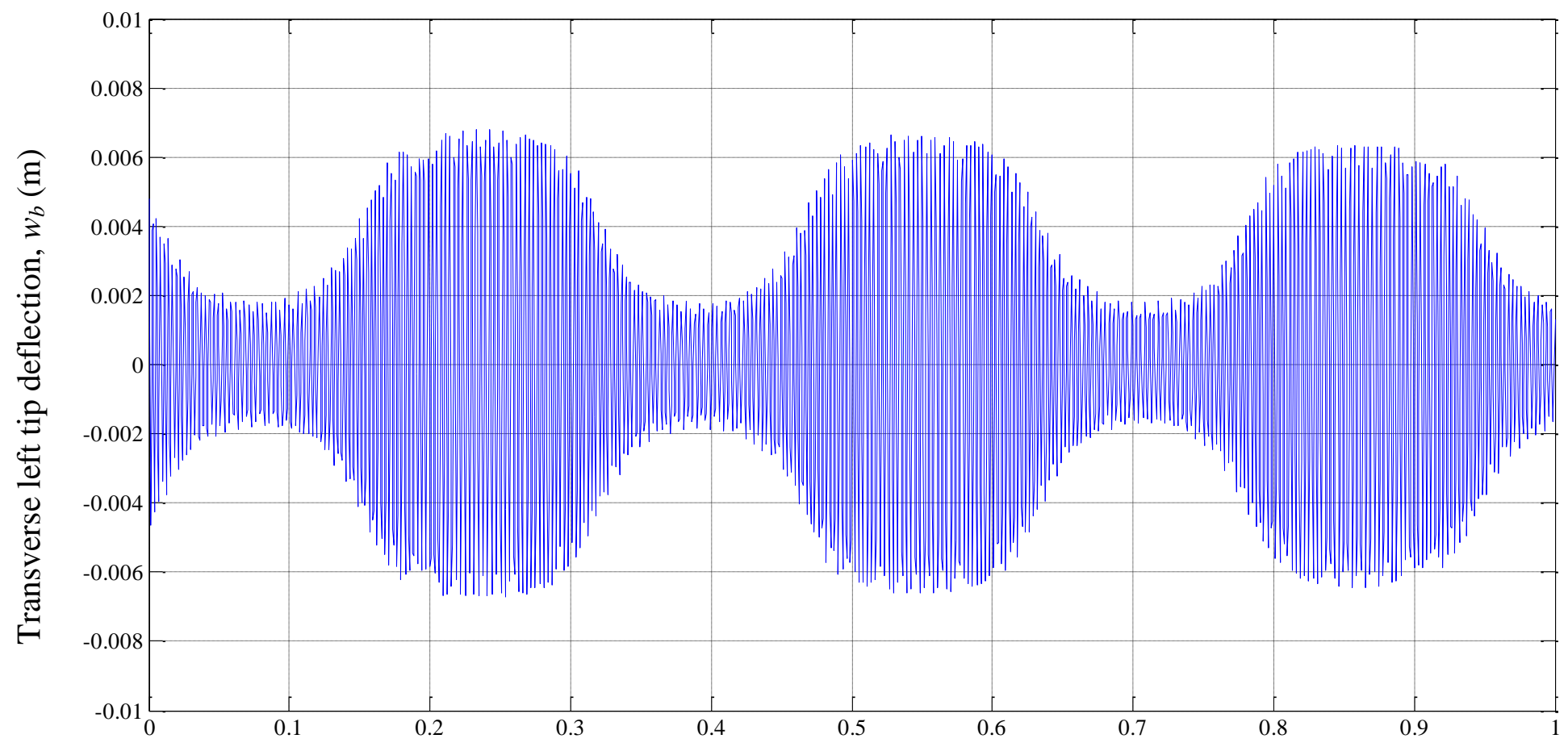

Time (s)

Figure 6.84 Transverse tip deflection at the left end, $w_{b}$, HSDT, $\Omega=20 \mathrm{rad} / \mathrm{s},[0 / \pm 45]_{\mathrm{s}}$ 


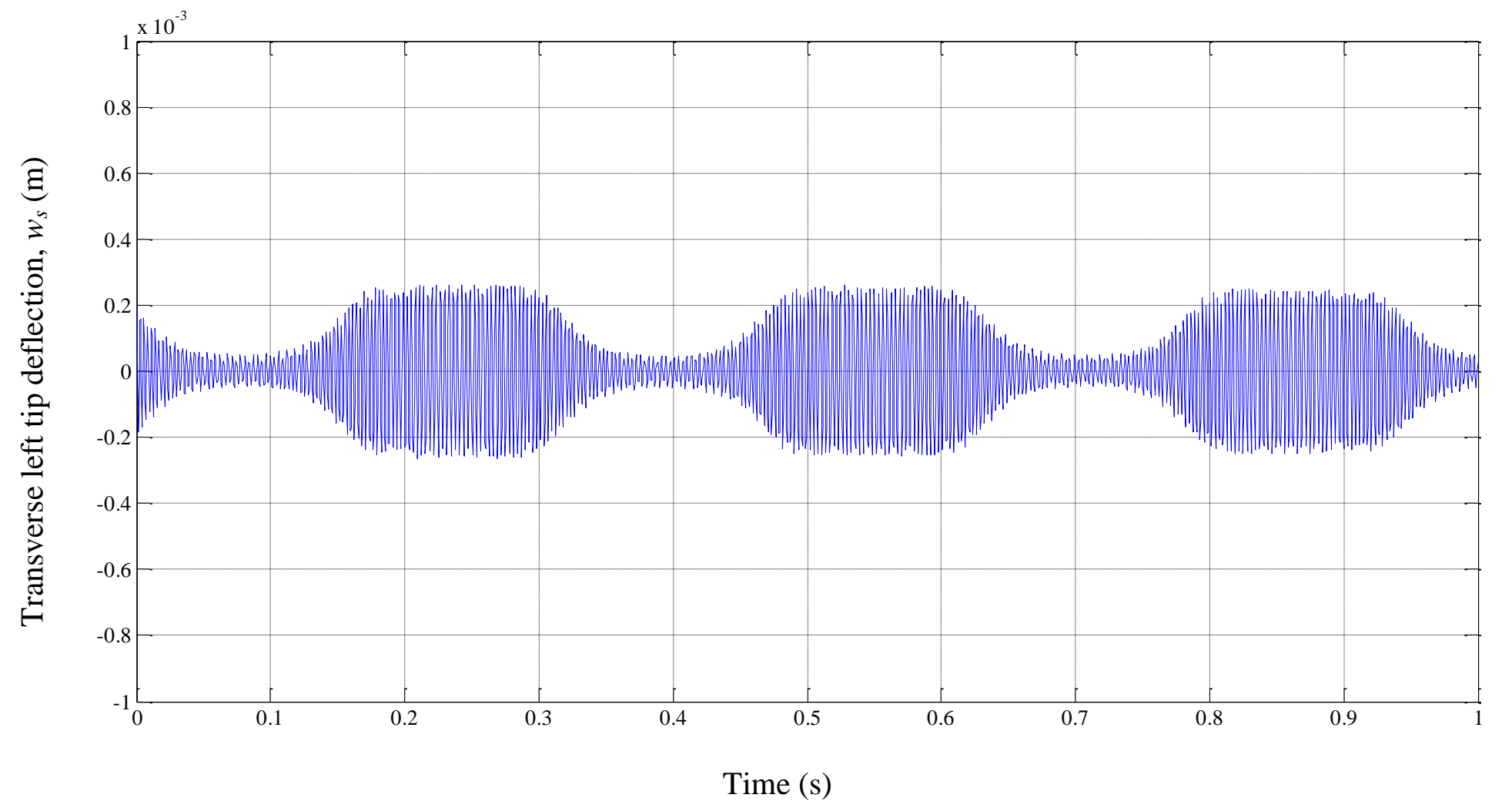

Figure 6.85 Transverse tip deflection at the left end, $w_{s}$, HSDT, $\Omega=20 \mathrm{rad} / \mathrm{s},[0 / \pm 45]_{\mathrm{s}}$ 


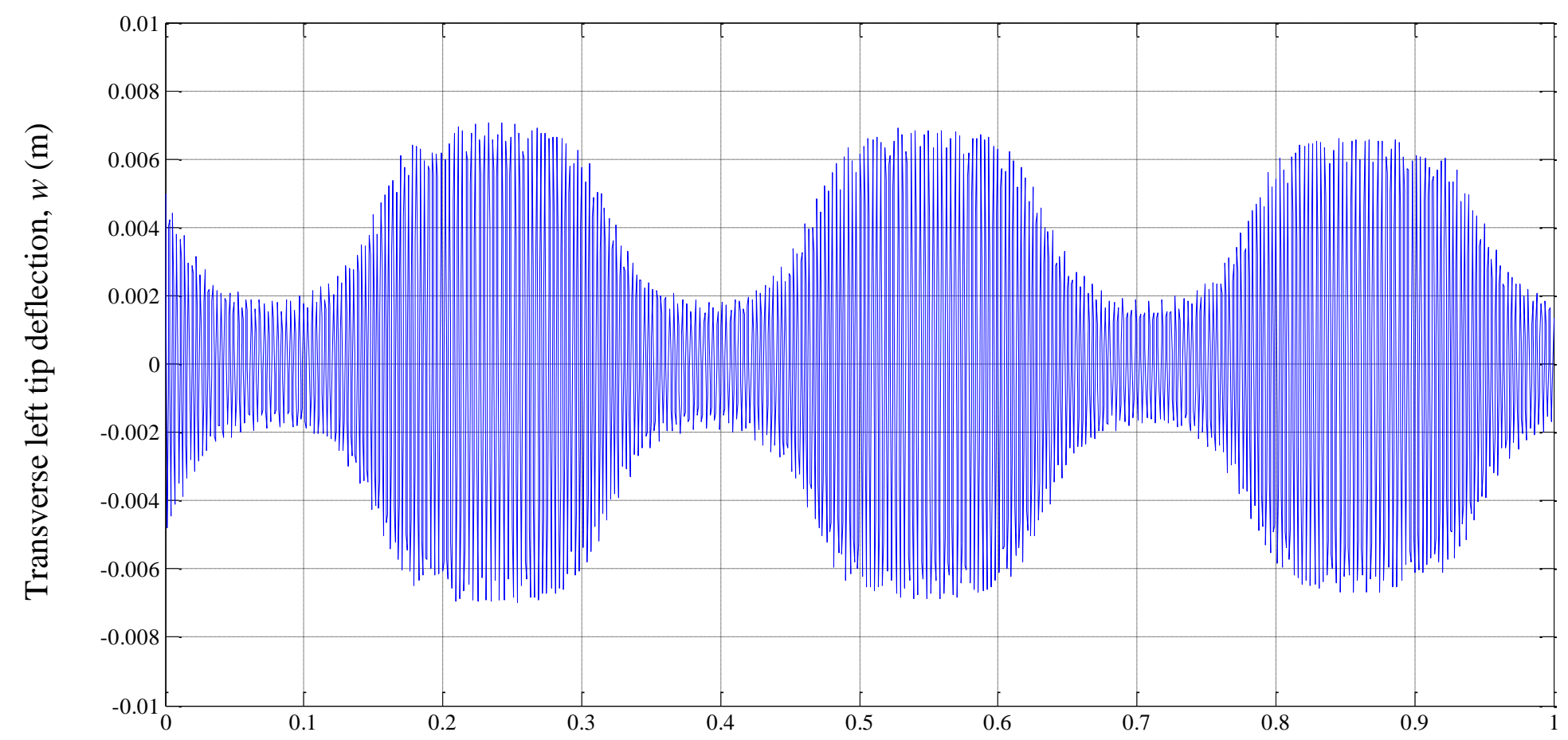

Time (s)

Figure 6.86 Transverse tip deflection at the left end, $w$, HSDT, $\Omega=20 \mathrm{rad} / \mathrm{s},[0 / \pm 45]_{\mathrm{s}}$ 


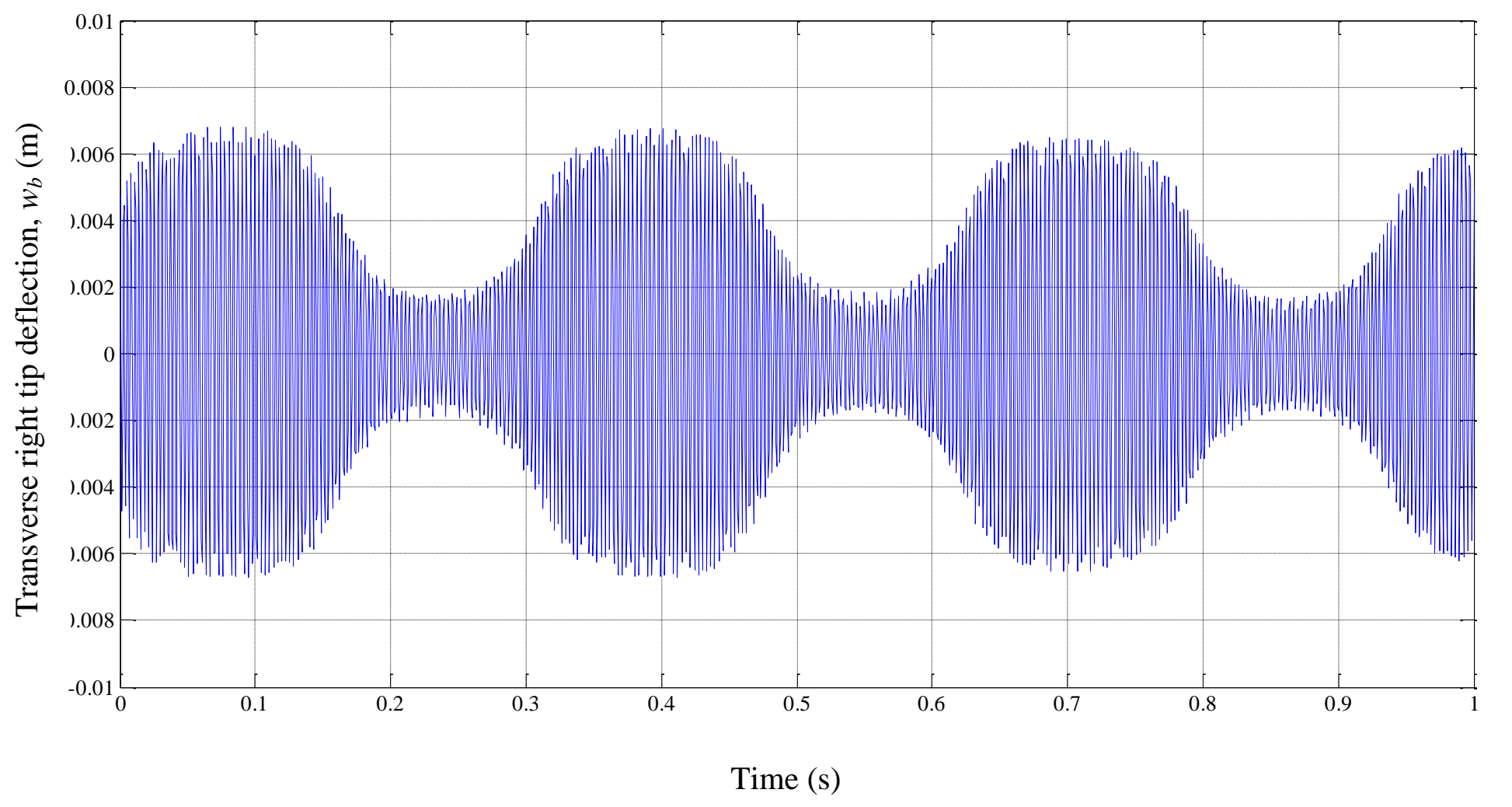

Figure 6.87 Transverse tip deflection at the right end, $w_{b}$, HSDT, $\Omega=20 \mathrm{rad} / \mathrm{s},[0 / \pm 45]_{\mathrm{s}}$ 


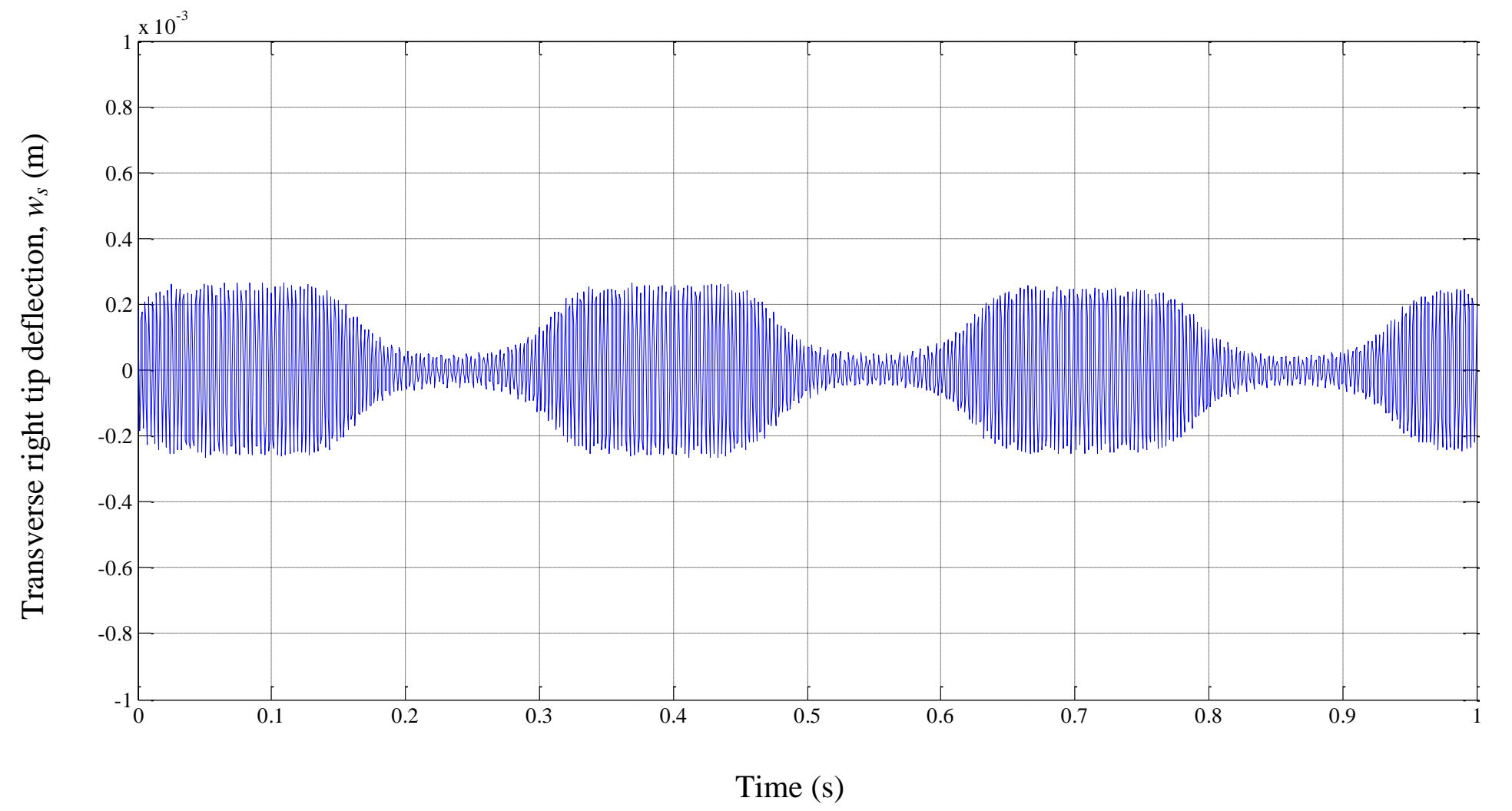

Figure 6.88 Transverse tip deflection at the right end, $w_{s}$, HSDT, $\Omega=20 \mathrm{rad} / \mathrm{s},[0 / \pm 45]_{\mathrm{s}}$ 


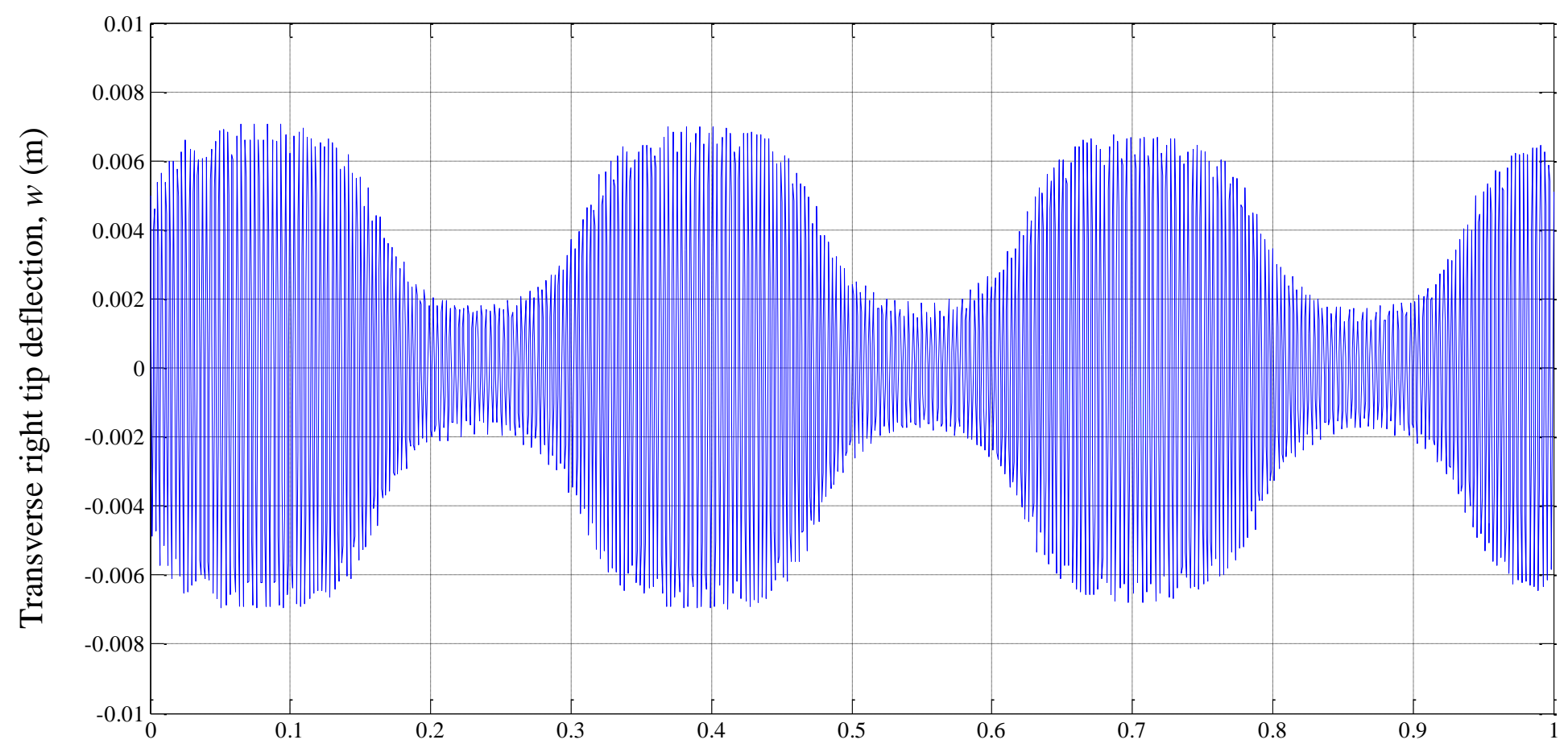

Time (s)

Figure 6.89 Transverse tip deflection at the right end, $w$, HSDT, $\Omega=20 \mathrm{rad} / \mathrm{s},[0 / \pm 45]_{\mathrm{s}}$ 


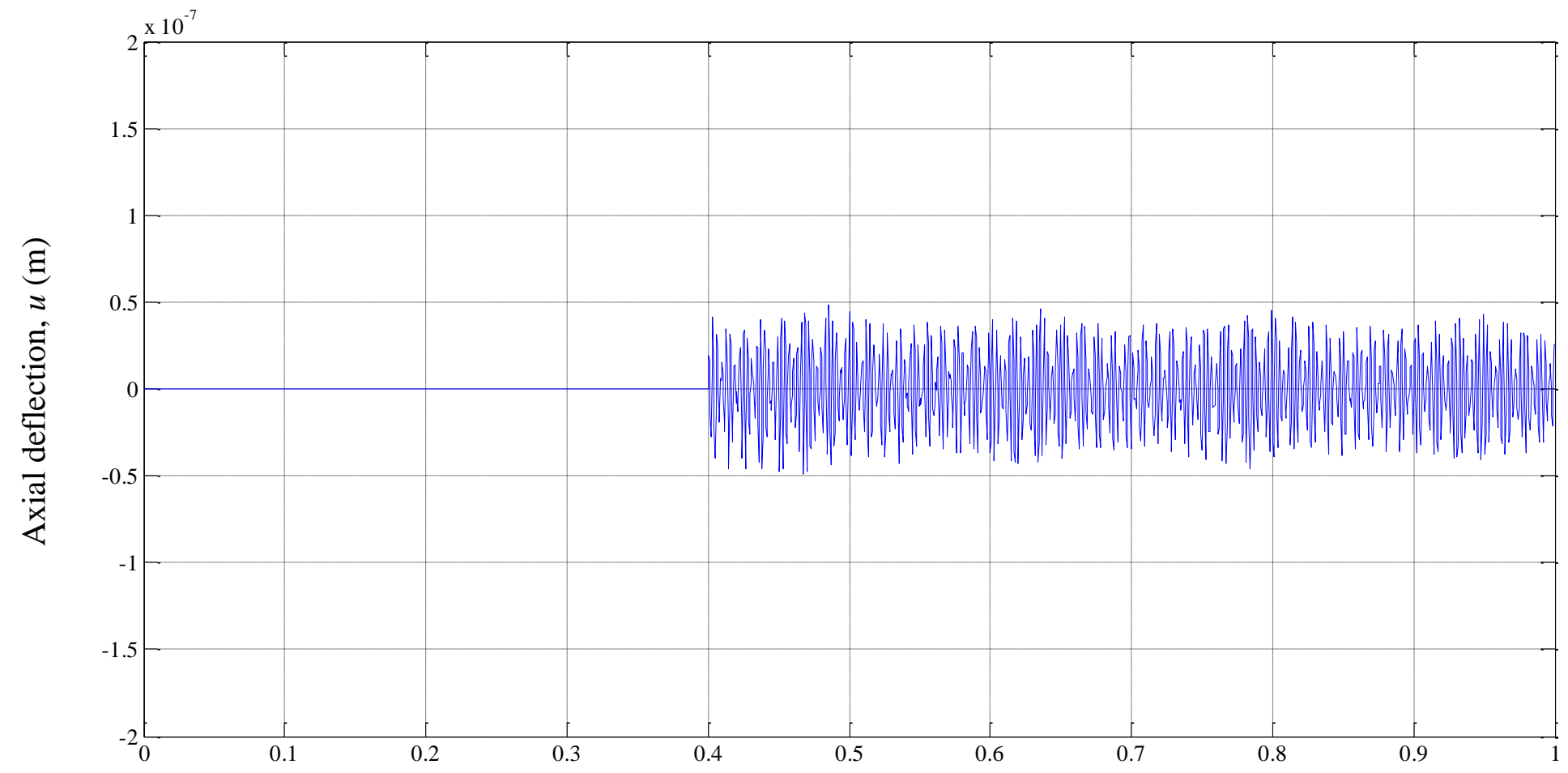

Time (s)

Figure 6.90 Axial deflection at the right end, $u$, HSDT, $\Omega=20 \mathrm{rad} / \mathrm{s},[0 / \pm 45]_{\mathrm{s}}$ 
Table 6.22 Transverse tip deflection at the left end, $w$, HSDT, $\Omega=20 \mathrm{rad} / \mathrm{s},[0 / \pm 45]_{\mathrm{s}}$

\begin{tabular}{|c|c|c|c|c|c|c|r|}
\hline$t(\mathrm{~s})$ & $w(\mathrm{~m})$ & $t(\mathrm{~s})$ & $w(\mathrm{~m})$ & $t(\mathrm{~s})$ & $w(\mathrm{~m})$ & $t(\mathrm{~s})$ & \multicolumn{1}{|c|}{$w(\mathrm{~m})$} \\
\hline 0.000000 & 0.005000 & 0.252000 & 0.007022 & 0.506500 & 0.006588 & 0.756500 & -0.002540 \\
\hline 0.012500 & -0.003894 & 0.267500 & 0.006921 & 0.512500 & 0.006655 & 0.765000 & -0.003187 \\
\hline 0.021000 & -0.003154 & 0.270500 & 0.006771 & 0.528000 & 0.006898 & 0.775000 & 0.003858 \\
\hline 0.030000 & -0.002551 & 0.281000 & -0.006701 & 0.539000 & -0.006874 & 0.789000 & 0.004883 \\
\hline 0.042000 & -0.002143 & 0.297000 & 0.006258 & 0.548500 & -0.006873 & 0.794500 & 0.005618 \\
\hline 0.052500 & 0.002117 & 0.305500 & 0.005854 & 0.558000 & -0.006857 & 0.803000 & 0.006043 \\
\hline 0.063500 & -0.001939 & 0.312500 & -0.005168 & 0.569000 & 0.006798 & 0.819000 & -0.006435 \\
\hline 0.073000 & -0.001864 & 0.325000 & 0.004059 & 0.570500 & -0.006838 & 0.829500 & 0.006527 \\
\hline 0.087000 & 0.001865 & 0.333500 & 0.003316 & 0.587500 & 0.006717 & 0.831000 & -0.006571 \\
\hline 0.096500 & 0.001987 & 0.341000 & -0.002690 & 0.593500 & 0.006632 & 0.846500 & -0.006664 \\
\hline 0.106000 & 0.002162 & 0.355500 & -0.002277 & 0.608000 & 0.006212 & 0.856000 & -0.006690 \\
\hline 0.118000 & 0.002348 & 0.366500 & 0.002066 & 0.611000 & 0.005958 & 0.865500 & -0.006691 \\
\hline 0.124000 & 0.002582 & 0.371000 & -0.002048 & 0.625000 & 0.005185 & 0.875000 & -0.006668 \\
\hline 0.138500 & 0.003180 & 0.380500 & -0.001923 & 0.630500 & 0.004583 & 0.886000 & 0.006546 \\
\hline 0.148500 & -0.004265 & 0.399500 & -0.001935 & 0.640500 & -0.003454 & 0.890500 & -0.006339 \\
\hline 0.157000 & -0.005043 & 0.409000 & -0.001978 & 0.656000 & 0.002670 & 0.906000 & -0.006119 \\
\hline 0.169500 & 0.006086 & 0.415000 & -0.001969 & 0.662000 & 0.002554 & 0.919000 & 0.005966 \\
\hline 0.179500 & -0.006469 & 0.424500 & -0.002221 & 0.674000 & 0.002157 & 0.920500 & -0.006099 \\
\hline 0.181000 & 0.006379 & 0.438000 & 0.002578 & 0.685000 & -0.001952 & 0.930500 & 0.005686 \\
\hline 0.194000 & -0.006193 & 0.444000 & 0.002851 & 0.699000 & 0.001862 & 0.943000 & -0.004555 \\
\hline 0.209500 & -0.006944 & 0.459500 & -0.003631 & 0.708500 & 0.001856 & 0.950000 & 0.004095 \\
\hline 0.211000 & 0.006943 & 0.469500 & 0.004853 & 0.718000 & 0.001894 & 0.960000 & -0.003268 \\
\hline 0.223500 & 0.007022 & 0.475000 & 0.005210 & 0.727500 & 0.001978 & 0.970000 & 0.002516 \\
\hline 0.233000 & 0.007048 & 0.489000 & 0.006054 & 0.738500 & -0.001975 & 0.980500 & -0.002217 \\
\hline 0.242500 & 0.007048 & 0.490500 & -0.006334 & 0.749000 & 0.002326 & 0.992500 & -0.001986 \\
\hline
\end{tabular}




\section{CONCLUSIONS AND RECOMMENDATIONS}

\subsection{Conclusions}

- A beat-like phenomenon is observed in the response of a composite moving beam and the number of beats increases with axial rigid body frequency.

- The numerical results indicate that the model incorporating piezoelectric sensors and actuators successfully reduces the vibrational response of composite beams.

- Gains used in the negative-velocity feedback system are varied over a wide range to obtain an optimum value that can reduce the amplitude of vibrations at the left end of the beam to the initial value for CLPT.

- Gains required to reduce the amplitude for a beam with [0/ 20$]$ layup is nearly ten times higher than that for the beam [0/ \pm 45$]$ layup.

- Damped response of moving beams formulated using FSDT and HSDT are obtained for the same gains as in CLPT. The beams with the HSDT formulation are observed to have the lowest damping ratio while the ones with the FSDT formulation have the highest damping ratio.

- Axial deflections due to the inertial forces generated by the rigid-body axial motion are negligible for symmetric and unsymmetric laminates.

- For a symmetric laminate, the axial deflection is appreciable only after the introduction of damping that couples the axial and transverse deflections.

- The right and the left end transverse deflections of the beam are seen to be out of phase. 


\subsection{Recommendations for Future Work}

- In addition to the active damping applied in the models of present research, material damping needs to be included.

- The numerical results can be validated experimentally.

- Nonlinear analysis based on large defection theory can be performed.

- The response of the piezoelectric smart moving beam under forced vibrations can be studied.

- Stress distribution along the length of the beam can be studied. 


\section{REFERENCES}

Balamurugan, V. and Narayanan, S., 2001, “Active Vibration Control of piezoelectric Smart Beams," Defence Science Journal, Vol. 51, pp. 103-114.

Barbero, E.J., 2010, “Introduction to Composite Materials Design,” Second Edition, Taylor and Francis, Philadelphia.

Bailey, T. and Hubbard, J.E., 1985, "Distributed Piezoelectric Polymer Active Vibration Control of a Cantilever Beam," Journal of Guidance, Control and Dynamics, Vol. 8, No. 5, pp. 605-611.

Bathe, K.J., 2001, “Finite Element Procedures,” Fifth Edition, Prentice-Hall of India Private Ltd., New Delhi, India.

Buffington, K.W. and Kane, T.R., 1985, "Dynamics of a Beam Moving Over Supports," International Journal of Solids and Structures, Vol. 21, No. 7, pp. 617-643.

Chang-Qing, C., Xiao-Ming, W., and Ya-Peng, S., 1996, "Finite Element Approach of Vibrational Control Using Self-Sensing Piezoelectric Actuators," Computers and Structures, Vol. 60, No. 3, pp. 505-512.

Chandrasekaran, G., 2000, "Dynamic Analysis of a Composite Moving Beam," Master's Thesis, West Virginia University, Morgantown, WV.

Detwiler, D.T., Shen, M.-H.H., and Venkayya, V.B., 1995, "Finite Element Analysis

of Laminated Composite Structures Containing Distributed Piezoelectric Actuators and Sensors," Finite Elements in Analysis and Design, Vol. 20, pp. 87-100.

Donthireddy, P. and Chandrashekhara, K., 1996, "Modeling and Shape Control of Composite Beams with Embedded piezoelectric actuators," Composite Structures, Vol. 35, pp. 237-244. 
Ha, S.K., Keilers, C. and Chang, F.K., 1992, "Finite Element Analysis of Composite Structures Containing Distributed Piezoceramic Sensors and Actuators," AIAA Journal, Vol. 30, No. 3, pp. 772-780.

Hamed, E.S. and Negm, H.M., 2004, "Finite Element Model for Smart Beams with Piezoelectric Actuators and/or Sensors," Journal of Engineering and Applied Science, Vol. 51, No. 3, pp. 575-592.

Koconis, D.B., 1993, "Shape Control of Composite Plates and Shells using Embedded Actuators," Ph.D Dissertation, Stanford Uniiversity, Stanford, CA.

Kadivar, M.H. and Mohebpour, S.R., 1998, "Finite Element Dynamic Analysis of Unsymmetric Composite Laminated Beams with Shear Effect and Rotary Inertia under the Action of Moving Loads," Finite elements in Analysis and Design, Vol. 29, pp. 259-273.

Kapania, R.K. and Raciti, S., 1989a, "Nonlinear Vibrations of Unsymmetrically Laminated Beams,” AIAA Journal, Vol. 27, No. 2, pp. 201-210.

Kapania, R.K. and Raciti, S., 1989b, "Recent Advances in Analysis of Laminated Beams and Plates. Part II. Vibrations and wave propagation," Enhancing Analysis Techniques for Composite Materials, NDE-Vol. 10, pp. 159-168.

Kapania, R.K. and Singhvi, S., 1991, "Free Vibration Analysis of Generally Laminated Tapered Skew Plates,” AIAA Journal, Vol. 27, No. 2, pp. 201-210.

Lee, C.K., 1990, "Theory of Laminated Piezoelectric Plates for the Design of Distributed Sensors/Actuators. Part I: Governing Equations and Reciprocal Relationships,” The Journal of the Acoustical Society of America, Vol. 87, No. 3, pp. 1144-1158. 
Lee, I., and Lee, J.J., 1990, "Vibration analysis of a composite plate wing," Journal of Computers and Structures, Vol. 36, No. 6, pp. 1077-1085.

Liew, K.M., He, X.Q., Tan, M.J., and Lim, H.K., 2004, "Dynamic Analysis of Laminated Composite Plates with Piezoelectric Sensors/Actuator Patches using the FSDT Mesh-free Method," International Journal of Mechanical Sciences, Vol. 46, pp. 411-431.

Marur, R.S., and Kant, T., 1998, “Transient Dynamics of Laminated Beams: an Evaluation with Higher-Order Refined Theories," Journal of Composite Structures, Vol.41, pp. 1-7.

Mitchell, J.A. and Reddy, J.N., 1994, “A Refined Hybrid Plate Theory for Composite Laminates with Piezoelectric Laminae," International Journal of Solids Structures, Vol. 32, pp. $2345-2367$.

Moita, J.M.S., Correia, I.F.P., Soares, C.M.M., and Soares, C.A.M., 2004, “Active Control of Adaptive Laminated Structures with Bonded Piezoelectric Sensors and Actuators," Computers and structures, Vol. 82, pp. 1349-1358.

Nagappan, G., 2004, “Analysis of Composite Moving Beams using Higher Order Shear Deformation Theory," Master's Thesis, West Virginia University, Morgantown, WV.

Narayanan, S. and Balamurugan, V., 2002, "Finite Element Modelling of Piezolaminated Smart Structures for Active Vibration Control with Distributed Sensors and Actuators," Journal of Sound and Vibration, Vol. 262, pp. 529-562.

Reddy, J.N., 1997, “On Laminated Composite Plates with Integrated Sensors and Actuators," Engineering Structures, Vol. 21, pp. 568-593.

Reddy, J.N., 2004, "Mechanics of Laminated Composite Plates: Theory and Analysis," Second Edition, CRC Press, Boca Raton, Fla. 
Saravanos, D.A and Heyliger, P.R., 1995, "Coupled layerwise analysis of Composite Beams with Embedded Piezoelectric Sensors and Actuators," Journal of Intelligent Materials and Structures, Vol. 6, No. 3, pp. 350-363.

Saravanos, D.A., Heyliger, P.R and Hopkins, D.A., 1995, "Layerwise Mechanics and Finite Element for the Dyanamic Analysis of Piezoelectric Composite Plates," International Journal of Solids Structures, Vol. 34, No. 3, pp. 359-378.

Singh, G., Rao, G.V., and Iyengar N.G.R., 1991, “Analysis of Nonlinear

Vibrations of Unsymmetrically Laminated Composite Beams," AIAA Journal, Vol. 29, No. 10, pp. 1727-1735.

Sivaneri, N.T. and Nagappan, G,, 2012, "Axially-Moving Composite Beams using Higher Order Shear Deformation Theory," 53rd AIAA/ASME/ASCE/AHS/ASC Structures, Structural Dynamics and Materials Conference, Honolulu, HA.

Sreeram, T.R. and Sivaneri, N.T., 1997, "FE-Analysis of Moving Beam using Lagrangian Multiplier Method," International Journal of Solids and tructures, Vol. 35, No. 28-29, pp. 3675-3694.

Sun, B. and Huang, Da., 2001, "Vibration Suppression of Laminated Composite Beams with a Piezo-electric Damping Layer," Composite Structures, Vol. 53, pp. 437-447.

Tzou, H.S., 1993, "Piezoelectric Shells: Distributed Sensing and Control of Continua," Kluwer Academic Publishers, Norwell, MA. 


\section{BIBLIOGRAPGHY}

Reddy, J.N., 1984, "Energy and variational methods in applied mechanics: with an introduction to the finite element method," Wiley, New York. 


\title{
Vibration Attenuation of Composite Moving Beams using Active Vibration Control Techniques
}

\section{Gouthami Polina}

\begin{abstract}
Thesis submitted to the
Statler College of Engineering and Mineral resources at the West Virginia University in partial fulfillment of the requirements for the Degree of
\end{abstract}

Master of Science

in

Mechanical Engineering

Department of Mechanical and Aerospace Engineering

APPROVAL OF THE EXAMINING COMMTTTEE

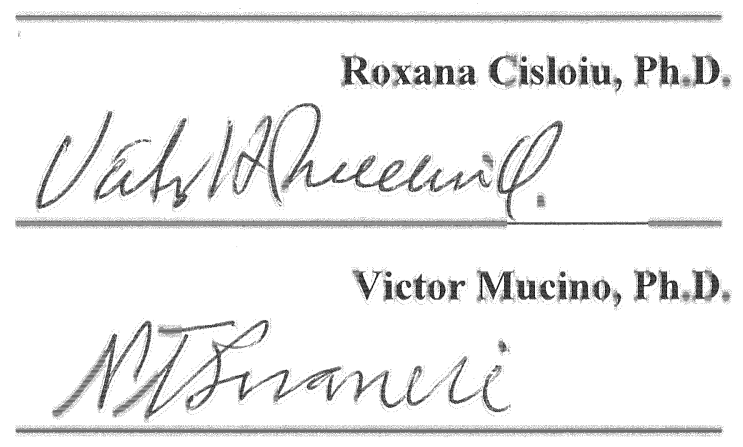

March 28, 2014

Nithi Sivaneri, $\mathbf{P h}, \mathbf{D}_{\text {, }}$ Chair

Date 